RONNy BERnd LUtz

THE SEARCH FOR SUBSTELLAR COMPANIONS TO SUBDWARF B STARS IN CONNECTION WITH EVOLUTIONARY ASPECTS

Dissertation

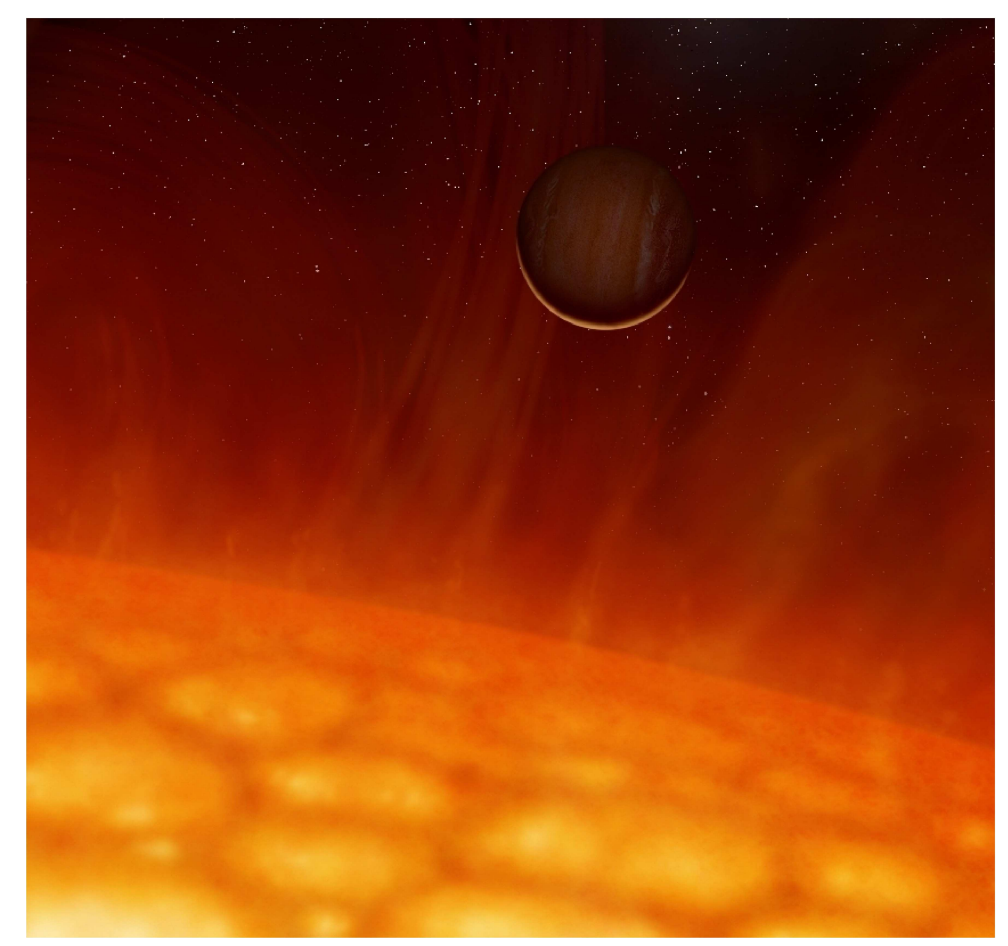


Cover illustration:

Image courtesy of HELAS, the European Helio- and Asteroseismology Network, funded by the European Union under Framework Programme 6;

Mark Garlick, artist. 


\title{
The search for substellar companions to subdwarf B stars in connection with evolutionary aspects
}

\author{
Dissertation \\ zur Erlangung des \\ mathematisch-naturwissenschaftlichen Doktorgrades \\ "Doctor rerum naturalium" \\ der Georg-August-Universität Göttingen
}

\author{
vorgelegt von \\ Ronny Bernd Lutz \\ aus Fürstenhagen
}

Göttingen, 2011 
Referentin:

Dr. Sonja Schuh

Korreferent:

Prof. Dr. Stefan Dreizler

Tag der mündlichen Prüfung:

27.09.2011 
Georg-August-Universität Göttingen

Institut für Astrophysik

International Max Planck Research School on Physical Processes in the Solar System and Beyond 



\section{Zusammenfassung}

Die Entstehung und Entwicklung heisser unterleuchtkräftiger B Sterne (im Folgenden sdB Sterne) ist nicht vollständig verstanden. Zwar kann der momentane Entwicklungszustand, also die Position im Hertzsprung-RussellDiagramm, recht gut charakterisiert und interpretiert werden, jedoch gibt es Unklarheiten bezüglich ihrer vorhergehenden Entwicklung. Da die massearmen sdB Sterne nicht dort vorzufinden sind, wo man es nach der Standardentwicklung massearmer Sterne erwarten würde, muss eine Phase oder ein Ereignis in deren Entwicklung stattgefunden haben, welches dieses Abweichen von der Standardentwicklung zur Folge hat. Die Literatur ist sich einig, dass diese Phase bzw. dieses Ereignis ein erhöhter Massenverlust des sdB Vorgängersterns ist, während dieser sich im Roter Riese Stadium befindet. Dies ist Voraussetzung um die Existenz der sdB Sterne zu erklären, jedoch ist die Ursache des erhöhten Massenverlustes nicht eindeutig geklärt. Im Falle der sdB Sterne als Teil eines Doppelsternsystems haben sich Szenarien der Entwicklung enger Doppelsternsysteme unter Berücksichtigung verschiedener Phasen von Roche-Lobe-Overflow und einer gemeinsamen Hülle beider Komponenten als erfolgsversprechend durchgesetzt. Hier stehen Theorie und Beobachtung recht gut in Einklang miteinander.

Probleme gibt es allerdings bei Einzelsternen, d. h. bei solchen sdB Sternen, die nicht Teil eines Doppelsystems sind, und keine stellaren Begleiter aufweisen. Solche einzelnen sdB Sterne sind Gegenstand dieser Arbeit. Eine in der Literatur diskutierte Hypothese besagt, dass selbst substellare Begleiter wie Braune Zwerge oder Exoplaneten einen entscheidenden Einfluss auf die Entstehung der Einzel-sdB Sterne haben können. Ein Hauptziel der vorliegenden Arbeit ist es, diese Hypothese zu untersuchen. Dies soll durch die Suche nach substellaren Begleitern um Einzel-sdB Sterne geschehen. Ein weiteres Hauptziel dieser Arbeit ist es, den Entwicklungsstatus der hier betrachteten sdB Sterne näher zu bestimmen und deren Entwicklungszeit- 
skalen direkt zu messen.

Die konkreten Ziele bezüglich der in dieser Arbeit untersuchten sdB Sterne sind nun die Suche nach substellaren Begleitern und die Bestimmung der Entwicklungszeitskalen der Sterne.

Um die oben genannten Ziele zu erreichen wurde das EXOTIME Programm ins Leben gerufen, welches fünf pulsierende sdB Sterne langfristig mit der Methode der bodengebundenen zeitaufgelösten relativen Photometrie beobachtet. Für zwei dieser fünf sdB Sterne, HS 0444+0458 und HS 0702+6043, wird in dieser Arbeit die Datenaufnahme sowie Reduktion, Auswertung und Interpretation dargestellt. Die für die Analyse notwendigen Daten setzen sich aus vielen Einzelmessungen zusammen, verteilt über einen Zeitraum von drei Jahren. Dies beruht auf der angewandten Methode, welche voraussetzt dass ein Objekt über einen möglichst langen Gesamtzeitraum gesehen so häufig wie möglich beobachtet wird. Der enorme Bedarf an photometrischen Daten lässt sich am besten mit koordinierten Kampagnen mehrerer beteiligter Observatorien bewältigen, so wie es auch Grundlage im EXOTIME Programm ist. Die im oben genannten Zeitraum angesammelte Menge an photometrischen Daten summiert sich auf annähernd 100 Stunden für den Stern HS 0444+0458 und zirka 850 Stunden für den Stern HS 0702+6043. Dazu haben insgesamt zwölf Observatorien beigetragen.

Um sowohl nach substellaren Begleitern zu suchen als auch die Entwicklungszeitskalen direkt zu messen, wird eine "Timing-Methode" verwendet. Dabei werden die gemessen Ankunftszeiten der Intensitätsmaxima, welche auf der periodischen Helligkeitsänderung des pulsierenden Sterns beruhen, mit aus einem Modell erwarteten Werten verglichen. Dadurch lassen sich wichtige Aussagen über das zeitliche Verhalten der Pulsationen machen, welche dann im Sinne der obigen Ziele und Fragestellungen interpretiert werden können.

Die im Rahmen dieser Arbeit gemessenen Charakteristika der Pulsationsperioden in HS 0444+0458 und HS 0702+6043 lassen wichtige Erkenntnisse bezüglich deren Entwicklungsstatus zu. Beide Objekte befinden sich in einer Phase der globalen Expansion, d.h. die Pulsationsperioden sind nicht konstant, sondern nehmen langfristig mit der Zeit zu. Die hier auftretenden Zeitskalen liegen im Bereich einer halben Million Jahre bzw. 1,8 Millionen Jahre für die zwei stärksten Pulsationen in HS 0444+0458 und bei etwa 40 
Millionen Jahren bzw. 2.2 Millionen Jahren für die zwei stärksten Pulsationen in HS 0702+6043. Ein Vergleich dieser Zeitskalen mit theoretischen Sternentwicklungs-Modellen lässt vermuten, dass die beiden untersuchten Sterne wahrscheinlich kurz vor dem Ende ihres Daseins als sdB Stern stehen. Damit hätten sie bereits die Phase des Heliumbrennens im Kern beendet und stünden kurz vor ihrer Entwicklung hin zur Abkühlsequenz Weisser Zwerge. Die in dieser Arbeit gemessenen Entwicklungszeitskalen und Charakteristika der Pulsationen stellen wichtige Bedingungen an theoretische sdB-Modelle der Asteroseismologie und können helfen die vorhandenen Modelle zu verfeinern.

Für die Fragestellungen dieser Arbeit treten als wichtigste Effekte mit Auswirkungen auf die stellaren Pulsationen der bereits erwähnte Einfluss der Sternentwicklung sowie der gravitative Einfluss eines hypothetischen Begleiters, welcher den sdB Stern umkreist, auf. Das Auftreten von Schwebungen als ein weiterer Effekt mit Einfluss auf die gemessenen Eigenschaften der Pulsationen wurde als solcher erkannt und entsprechend von den anderen Effekten getrennt.

Die Entdeckung substellarer Begleiter um beide hier untersuchten Sterne stellt ein zentrales Ergebnis dieser Arbeit dar und lässt wichtige Rückschlüsse bezüglich der weiter oben erwähnten Entstehungsszenarien zu. Mit einer Mindestmasse von etwa 30-45 Jupitermassen kreist der Braune Zwerg Kandidat HS 0444+0458 b in einem Abstand von 0.27 Astronomischen Einheiten um seinen Zentralstern HS 0444+0458. Der exoplanetare Kandidat HS 0702+6043 b hat eine Mindestmasse von 5-6 Jupitermassen und befindet sich in einem Abstand von etwa 1.15 Astronomischen Einheiten von seinem Zentralstern HS 0702+6043. Die gemessenen Parameter stehen im Einklang mit Modellen, welche voraussagen, dass diese beiden Objekte in der Lage waren, die Entwicklung ihrer Muttersterne zu beeinflussen. Der Einfluss der Begleiter kann einen erhöhten Massenverlust, welcher benötigt wird um die Existenz von sdB Sternen zu erklären, hervorgerufen haben. Die Hypothese des direkten Einflusses substellarer Begleiter auf die Entstehung von Einzel-sdB Sternen wird durch die hohe Detektionsrate gefundener Begleiter gestärkt. Bei jedem der drei hinreichend untersuchten EXOTIME Objekte HS 2201+2610, HS 0444+0458 und HS 0702+6043 (letztere beide als Gegenstand der vorliegenden Arbeit) kann ein substellarer Begleiter mit 
der "Timing Methode" nachgewiesen werden. Dies ist eine positive Tendenz, um jedoch statistisch signifikante Aussagen zu machen, bedarf es einer grösseren Anzahl von Einzel-sdB Sternen die nach substellaren Begleitern untersucht werden. Die hier präsentierten Resultate sollten zu solch einer ausgedehnten Suche motivieren, um letztendlich die Hypothese des Einflusses substellarer Begleiter auf die Entstehung von sdB Sternen weiter zu festigen.

Diese Arbeit ist folgendermaßen aufgebaut:

Das erste Kapitel stellt den momentanen Stand der Forschung bezüglich der sdB Sterne vor und liefert die nötigen Hintergrundinformationen, sowohl experimentell als auch theoretisch, um dem Kurs dieser Arbeit zu folgen. Anschliessend werde ich im zweiten Kapitel die grundlegenden Fragestellungen und Zielsetzungen kurz motivieren, welche letztendlich in der Idee zur Formulierung dieser Arbeit resultierten und auf die Untersuchung von sdB Sternen im Hinblick auf substellare Begleiter zielen. Das dritte Kapitel erläutert die Zielsetzungen des EXOTIME Projektes und wird die in dieser Arbeit untersuchten Sterne näher charakterisieren. Im Anschluss wird im vierten Kapitel das Archiv der Beobachtungsdaten, welche im Laufe der vergangenen Jahre für diese Arbeit aufgenommen wurden, präsentiert. Dabei wird vertieft auf die beteiligten Observatorien und auf die Beobachtungsstrategie eingegangen. Im fünften Kapitel werden die Beobachtungsmethoden zur Suche nach substellaren Begleitern um sdB Sterne eingehend erläutert. Hier soll besonders ein Vergleich der verwendeten "Timing-Methode" zu verschiedenen anderen Detektionsmethoden, welche ebenso auf die Suche nach Exoplaneten abzielen, gezogen werden. Die Ergebnisse der Analyse werden ausführlich im sechsten Kapitel dargestellt. Im Weiteren werden diese Ergebnisse in Kapitel 7 diskutiert und in einen grösseren Zusammenhang gebracht. Dabei wird sowohl auf Schlussfolgerungen als auch auf Anwendungen vertieft eingegangen. Eine Zusammenfassung und ein Ausblick, wie in Kapitel 8 dargestellt, werden diese Arbeit abschliessen. 


\section{Abstract}

The formation and evolution of hot subluminous B stars (sdB stars in the following) is not entirely understood. Although the current evolutionary status, i. e. the position in the Hertzsprung-Russell diagram, can be characterized and interpreted quite well, the preceding evolution of these objects remains uncertain. Since sdB stars do not follow a standard evolution of low mass stars, there must have been some event or phase in their history which led to a departure of the standard description. It is well-established in the literature that this event is connected to an enhanced mass loss of the sdB progenitor star during its red giant phase. This enhanced mass loss is a prerequisite to explain the very existence of sdB stars, its cause is, however, matter of debate. In the case of subdwarf B stars in binary systems, formation scenarios invoking Roche-lobe overflow and common envelope evolution are most promising. Theory and observations agree quite well in this case. However, there are some issues concerning single sdB stars, i. e. such stars which are not part of a binary system and therefore do not have a stellar companion. Such single sdB stars are an integral part of this thesis. One particular hypothesis discussed in the literature predicts that even substellar companions like brown dwarfs or exoplanets may be able to have a decisive influence on the formation of single sdB stars. A major goal of this present thesis is to investigate this hypothesis by searching for substellar companions to subdwarf B stars. Another goal is to pin down the evolutionary status of the investigated sdB stars and to directly measure their evolutionary timescales.

The precise purposes are hence the search for substellar companions and the determination of evolutionary timescales.

To achieve the goals mentioned above, the so-called EXOTIME program has been set up. EXOTIME conducts a long-term monitoring of five pulsating sdB stars with means of ground based time-resolved relative photometry. 
The observations, data reduction, analysis and interpretation for two of these five targets, namely HS $0444+0458$ and HS $0702+6043$, will be presented in this thesis. The observational data necessary for the analysis are comprised of many individual data sets gathered over the course of three years. This is due to the applied detection method, which demands that a target is observed as often as possible during a total timebase as long as possible. This need of huge amounts of photometric data can best be realized with coordinated campaigns with several participating observatories. This multi-site approach is also an integral design of the EXOTIME program. The amount of photometric data gathered during the time span mentioned above sums up to almost 100 hours for the target HS 0444+0458 and roughly 850 hours for the target HS 0702+6043. A total of twelve observatories have been involved in this process.

In order to search for substellar companions and to directly measure the evolutionary timescales, a timing method is applied. The measured arrival times of the intensity maxima, which result from periodic changes in the luminosity of the pulsating star, are compared to predicted arrival times calculated from a model. This allows to draw important conclusions for the temporal behavior of the pulsations, which can subsequently be interpreted in the context of the goals mentioned above.

In the framework of this thesis, the measured characteristics of the pulsation periods in HS 0444+0458 and HS 0702+6043 result in a fundamental insight in their evolutionary status. Both stars are in a phase of global expansion, i. e. the pulsation periods are not constant but increase with time. The timescales arising for the two strongest pulsations in HS 0444+0458 are 0.5 million years and 1.8 million years, respectively. For HS 0702+6043 they are roughly 40 million years and 2.2 million years for the two strongest pulsations, respectively. A comparison of these timescales to theoretical models of stellar evolution suggests that these two stars are close to the end of their sdB lifetime. If so, they would already have experienced exhaustion of core helium burning and would hence be close before evolving towards the white dwarf cooling sequence. The measured evolutionary timescales and characteristics of the pulsations, as central results of this thesis, can provide important constraints for theoretical asteroseismological sdB models and help to improve the present ones. 
With respect to the goals of this thesis, the most important effects with consequences on the stellar pulsations are stellar evolution as well as the gravitational influence of a hypothetical companion orbiting the sdB star. The presence of beating of close frequencies as a further effect with influence on the measured characteristics of the pulsations has been recognized as such and separated from the other effects accordingly.

The detection of substellar companions to both of the targets analyzed is a central result of this thesis and allows to draw important conclusions concerning the evolution scenarios mentioned earlier. Having a minimum mass of roughly 30-45 Jupiter masses, the brown dwarf candidate HS 0444+0458 b orbits its host star HS $0444+0458$ at a distance of 0.27 astronomical units. The exoplanet candidate HS $0702+6043 \mathrm{~b}$ has a minimum mass of 5-6 Jupiter masses and is separated from its host star HS $0702+6043$ by roughly 1.15 astronomical units. These measured parameter are in accordance with models which predict that both these substellar objects were indeed able to influence the formation and evolution of their host stars. The influence of the substellar companions can have caused an enhanced mass loss of the host stars, which is necessary to explain the existence of sdB stars. The hypothesis of this direct influence of the substellar companions on the formation of single sdB stars is supported by a high detection rate of companions. For each of the three EXOTIME targets which have been analyzed sufficiently (HS 2201+2610, HS 0444+0458 and HS 0702+6043, with the latter two being part of the present thesis), a substellar companion has been found with a timing method. This is a positive tendency. To be able to draw statistically significant conclusions, however, more single sdB stars will have to be analyzed in terms of possible substellar companions. Nevertheless, the results presented in this thesis should be very motivating to push forward the search for substellar companions to single sdB stars to finally strengthen the hypothesis of the ability of substellar companions to influence the formation of subdwarf B stars.

This thesis is organized as follows:

The first chapter introduces the current state in subdwarf B star research and provides the necessary background, both observationally and theoretically, to follow the course of this work. Afterwards, in the second chapter 
I will motivate the basic questions and intentions which led to the idea of setting up this thesis and to investigate the subdwarf B stars with respect to substellar companions. Chapter three is intended to introduce the purpose and goals of the EXOTIME project and to briefly characterize the target stars central to this thesis. Subsequently, in chapter four I will present the data archive built up during the last years, with a special emphasis on the observation facilities and observational strategy. Chapter five provides a description of the methods being applied to investigate the subdwarf B stars for substellar companions. A focus is set on the $\mathrm{O}-\mathrm{C}$ timing method and a comparison to various other exoplanet detection methods. The results of the analysis are extensively presented in chapter six. Chapter seven will discuss these results, put them into a broader context and address various other implications and applications. A summary and outlook, as provided in chapter eight, will conclude this thesis. 


\section{Contents}

1 Introduction 1

1.1 Stellar evolution . . . . . . . . . . . . . . . . . . . 2

$1.2 \mathrm{SdB}$ stars $\ldots \ldots \ldots \ldots \ldots . \ldots \ldots$

1.2.1 Formation and evolution . . . . . . . . . . . 8

1.3 Asteroseismologv . . . . . . . . . . . . . . . . . . . 14

1.3 .1 Stellar pulsations . . . . . . . . . . . . . . . 14

1.3 .2 SdBV stars . . . . . . . . . . . . . . . 23

1.4 Exoplanets . . . . . . . . . . . . . . . . . . . 27

2 Motivation 35

$\begin{array}{lll}3 & \text { EXOTIME } & 39\end{array}$

3.1 Description and status of the project . . . . . . . . . . 40

3.2 Targets . . . . . . . . . . . . . . . . . . . 40

3.2 .1 IR colors . . . . . . . . . . . . . . . . . . 45

3.2.2 Hybrid pulsations . . . . . . . . . . . . . . 50

4 Data

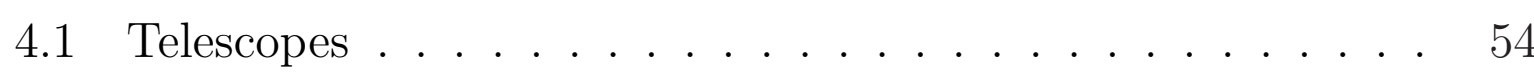

4.1.1 The MONET $/ \mathrm{N}$ telescope . . . . . . . . . . . . 57

4.2 Data archive . . . . . . . . . . . . . . . . 60

$\begin{array}{lll}5 \text { Methods } & 75\end{array}$

5.1 Time-series photometry . . . . . . . . . . . . . . . . 75

5.2 Time corrections . . . . . . . . . . . . . 76

5.3 Time-series and frequency analysis . . . . . . . . . . . . . 82

5.4 Timing Method . . . . . . . . . . . . . . . . . . . . . 83

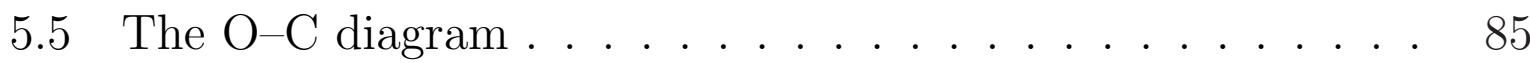


5.5.1 A linearly changing period . . . . . . . . . . 85

5.5.2 The orbital light-time effect . . . . . . . . . . 88

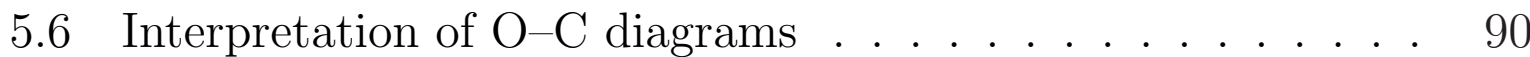

5.6 .1 The case of HS $2201+2610 \ldots \ldots$. . . . . . . . . 92

5.7 Sensitivity of the Timing Method . . . . . . . . . . . . 96

6 Analvsis and results: HS 0444 +0458 and HS 0702+6043 107

6.1 Light curves and periodograms . . . . . . . . . . . . . 107

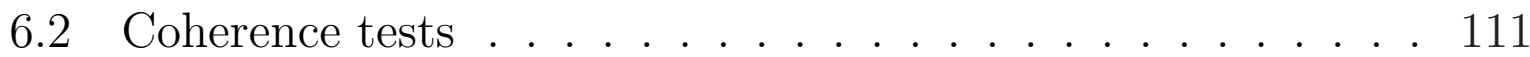

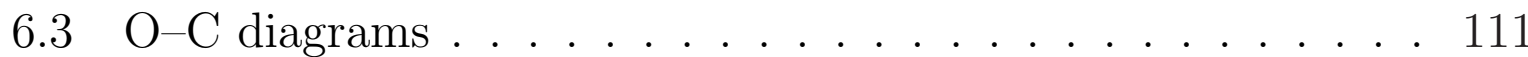

6.3.1 Evolutionarv signals . . . . . . . . . . . . . . 114

6.3 .2 Beating signals . . . . . . . . . . . . . . . . . 117

6.3 .3 Orbital signals . . . . . . . . . . . . . . . . . . . . 124

$6.3 .4 \quad \mathrm{O}-\mathrm{C}$ residuals . . . . . . . . . . . . . . 133

6.4 Detection limits for our data sets . . . . . . . . . . . . . . . 134

6.5 Current status for the other EXOTIME targets . . . . . . . 134

6.5 .1 HS $2201+2610 \ldots \ldots \ldots$

$6.5 .2 \quad \mathrm{PG} 1325+101 \ldots \ldots \ldots$

$6.5 .3 \quad$ EC 09582-1137 . . . . . . . . . . . . . 137

7 Discussion

7.1 Alternative interpretations and possible misinterpretations . 139

7.1.1 Treatment of the beating . . . . . . . . . . . . . . . . 141

7.1.2 The HS 0702+6043 "sin" signal . . . . . . . . . . . . . 142

7.1.3 Assumption of circular orbits . . . . . . . . . . . . . 143

7.2 Characteristics of the companion candidates . . . . . . . . 143

7.2.1 Overview of companion candidates . . . . . . . . . 143

7.2.2 Constraints on companion masses . . . . . . . . . . . 144

7.2.3 Predicted radial velocity signals . . . . . . . . . . . . 148

7.2.4 Prediction of $\mathrm{O}-\mathrm{C}$ points . . . . . . . . . . . . . 148

7.2.5 Effective temperatures of the companion candidates . 149

7.3 Stellar evolutionary context . . . . . . . . . . . . . 151

7.3.1 Common envelope evolution and final separation . . . 151

7.3.2 Evolutionarv status of the sdBs . . . . . . . 156

7.3.3 Impact of the companion candidates on sdB evolution 162

7.3.4 Future of solar-like host stars . . . . . . . . . . . . . 164 
8 Summary and outlook $\quad 167$

8.1 Summary . . . . . . . . . . . . . . . . . . 167

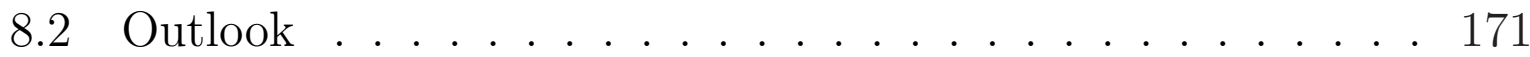

Bibliography $\quad$ I

$\begin{array}{lll}\text { Appendices } & \text { XIII }\end{array}$

A Finding charts and field of view snapshots $\quad$ XIII

B Example light curves $\quad$ XIX

$\begin{array}{ll}\text { C Example periodograms } & \text { XXIII }\end{array}$

D List of substellar companions to sdB stars $\quad$ XXV 



\section{List of Figures}

1.1 Hertzsprung-Russell diagram . . . . . . . . . . . . . . . . . 2

1.2 Horizontal Branch . . . . . . . . . . . . . . . . . . . . . . . . . . . . 5

$1.3 \quad$ SdB masses $\ldots \ldots \ldots \ldots$

1.4 Abundances $\ldots \ldots \ldots \ldots \ldots \ldots$

1.5 Pure vs. composite spectral ............. . . . 8

1.6 Formation scenarios: binary channels . . . . . . . . . . . . 10

1.7 Formation scenarios: single star channels . . . . . . . . . . 12

1.8 Hot flasher scenarios . . . . . . . . . . . . . . . . 13

1.9 Pulsational Hertzsprung-Russell diagram . . . . . . . . . . . 24

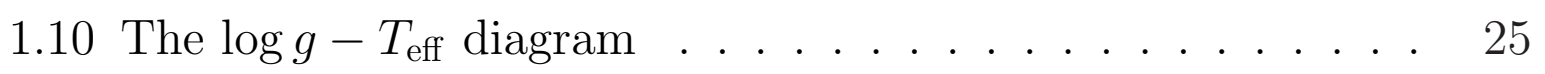

1.11 Comparison of various detection methods . . . . . . . . . . . 29

1.12 Exoplanet host stars . . . . . . . . . . . . . . 30

3.1 Target visibilities . . . . . . . . . . . . . . . . . . . 42

3.2 Blackbody and colors, HS 0444+0458 . . . . . . . . . . . 46

3.3 Blackbody and colors, HS 0702+6043 and PG 1325+101 . . . 47

3.4 Blackbody and colors, HS 2201+2610 and EC 09582-1137 . . 48

3.5 Signatures of hypothetical stellar companions . . . . . . . 51

4.1 EXOTIME telescopes . . . . . . . . . . . . . . . . . . 55

4.2 EXOTIME telescope contributions . . . . . . . . . . 56

4.3 Data archive for HS 0444+0458 . . . . . . . . . . . 57

4.4 Data archive for HS $0702+6043 \ldots \ldots \ldots$

4.5 MONET/N EXOTIME contributions . . . . . . . . . . . 59

5.1 Time standards . . . . . . . . . . . . . . . . . . . . 77

5.2 Leap seconds . . . . . . . . . . . . . . . . . . . 78

5.3 Flowchart for the time corrections . . . . . . . . . . . . 79 
5.4 Time corrections for our data . . . . . . . . . . . 80

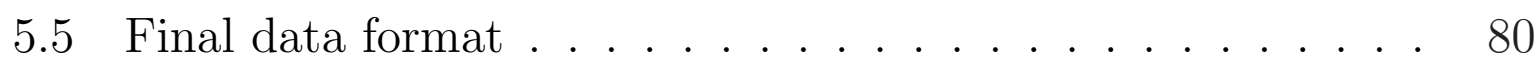

5.6 Principle of the $\mathrm{O}-\mathrm{C}$ method $\ldots \ldots \ldots \ldots$. . . . . . . 84

5.7 Orbital configuration . . . . . . . . . . . . . . . . . . 88

5.8 O-C diagram: different cases . . . . . . . . . . . . . . . . . . . 91

5.9 O-C diagram: construction . . . . . . . . . . . . . . . 92

5.10 O-C diagram: from phase- to time differences $\ldots . . . .9 .93$

5.11 O-C diagram: case of companion . . . . . . . . . . . 94

5.12 O-C diagrams of HS $2201+2610 \ldots \ldots \ldots \ldots$

5.13 Comparison of the timing method to other detection methods 97

5.14 Detection methods . . . . . . . . . . . . . . . . . . . . . . 98

5.15 Sensitivity of the timing method . . . . . . . . . . . . 99

5.16 Sensitivity of the timing method, logarithmic scales . . . . . 100

5.17 Sensitivity of the timing method, orbital sketch . . . . . . . 101

5.18 O-C amplitudes of hvpothetical close stellar companions . . 102

5.19 Orbital periods of hypothetical close stellar companions . . . 103

5.20 Timing sensitivity for the Solar System . . . . . . . . . . . . 104

6.1 Fourier Transforms for HS 0444+0458 and HS 0702+6043 . . 108

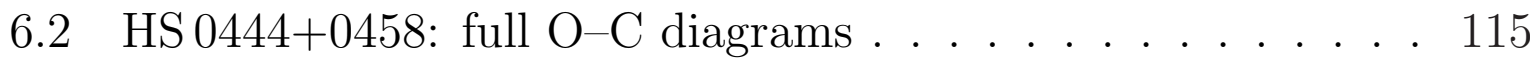

6.3 HS 0702+6043: full $\mathrm{O}-\mathrm{C}$ diagrams . . . . . . . . . . . 116

6.4 HS 0444+0458: O-C diagrams. Evolutionarv signals subtracted 118

6.5 HS 0702+6043: O-C diagrams. Evolutionary signals subtracted 119

6.6 Beating example . . . . . . . . . . . . . . . . . . 120

6.7 Beatings in $\mathrm{f} 1$ and $\mathrm{f} 2$ of HS 0444+0458 . . . . . . . . . 121

6.8 Beatings in $\mathrm{f} 1$ and $\mathrm{f} 2$ in HS 0702+6043 . . . . . . . . . . 122

6.9 HS 0444+0458: O-C diagrams. Evolutionary and beating signals subtracted . . . . . . . . . . . . 125

6.10 HS 0702+6043: $\mathrm{O}-\mathrm{C}$ diagrams. Evolutionary and beating signals subtracted . . . . . . . . . . . . . . 126

6.11 Fitted orbits . . . . . . . . . . . . . . . . . . . 127

6.12 Companion candidates . . . . . . . . . . . . . . . . 128

6.13 Phase-folded O-C. HS 0444+0458 . . . . . . . . . . . . . 129

6.14 Phase-folded O-C. HS 0702+6043 . . . . . . . . . . . . 130

$6.15 \mathrm{O}-\mathrm{C}$ residuals for $\mathrm{f} 1$ and $\mathrm{f} 2 \ldots \ldots \ldots . \ldots . \ldots . \ldots 133$

6.16 Detection limits for our datal . . . . . . . . . . . . . . . 135 
7.1 True companion masses.

145

7.2 Valid regimes for envelope masses and red giant radii. part a 154

7.3 Valid regimes for envelope masses and red giant radii, part b 155

7.4 Trpical evolutionary track of a sdB star . . . . . . . . . 157

7.5 Typical expansion timescales for a sdB star. . . . . . . . . 158

8.1 Summary of the O-C components . . . . . . . . . . . 170

A.1 Finding chart for HS 0702+6043 . . . . . . . . . . . . . XIV

A.2 Finding chart for HS 0444+0458 . . . . . . . . . . . . . XV

A.3 Finding chart for PG $1325+101$. . . . . . . . . . . . . . XVI

A.4 Finding chart for HS $2201+2610$. . . . . . . . . . . . . . . XVII

A.5 Finding chart for EC 09582-1137 . . . . . . . . . . . . XVIII

B.1 Light curve examples for HS 0444+0458 . . . . . . . . . . . . XX

B.2 Light curve examples for HS 0702+6043. part a . . . . . . . XXI

B.3 Light curve example HS 0702+6043, part b . . . . . . . . . . XXII

C.1 FT examples for HS 0702+6043 . . . . . . . . . . . XXIV 



\section{List of Tables}

1.1 Group properties of sdB pulsators . . . . . . . . . . . . . . . 26

3.1 EXOTIME targets . . . . . . . . . . . . . . . . . . . . . 44

3.2 Distance determination . . . . . . . . . . . . . . . . . . 49

4.1 Telescope site shortcuts for Tables 4.3-4.13. . . . . . . . . 61

4.2 Observer shortcuts for Tables 4.3-4.13. . . . . . . . . . . 62

4.3 Photometric data archive for HS0702+6043. part a. . . . . . 63

4.4 Photometric data archive for HS0702+6043. part b. . . . . . 64

4.5 Photometric data archive for $\mathrm{HS} 0702+6043$. part c. . . . . 65

4.6 Photometric data archive for HS0702+6043. part d. . . . . 66

4.7 Photometric data archive for HS0702+6043. part e. . . . . . 67

4.8 Photometric data archive for HS0444+0458. part a. . . . . . 68

4.9 Photometric data archive for HS0444+0458. part b. . . . . . 69

4.10 MONET and Calar Alto data archive for HS2201+2610. . . 70

4.11 Photometric data archive for PG1325+101, part a. . . . . . 71

4.12 Photometric data archive for PG1325+101. part b. . . . . . 72

4.13 Photometric data archive for EC09582-1137. . . . . . . . . 73

6.1 Fitting results part a . . . . . . . . . . . . . . . . 131

6.2 Fitting results, part b . . . . . . . . . . . . . . . . . 132

7.1 Unknown inclination . . . . . . . . . . . . . . . . 146

D.1 Collection of substellar companions to sdB stars . . . . . . . XXVI

D.2 Collection of controversial companions to sdB stars . . . . . XXVII 

"Astronomers, like burglars and jazz musicians, operate best at night."

- Miles Kington

Welcome to Kington, 1989 



\section{Chapter 1}

\section{Introduction}

Are there planets outside our own Solar System? The answer to this question is of course a definite "Yes!". And we are also able to do statistics on these exoplanets since the number of detections is continuously increasing. But how about the questions How do these exoplanetary systems evolve? or What is the fate of these systems? Our current knowledge is yet far from answering these questions unambiguously.

The vast majority of the more than 500 exoplanets known today is found around non-evolved solar-like stars. To gain more insight in the two latter questions, this thesis intends to take a closer look on a particular type of evolved stars, the subdwarf B ( sdB) stars. I will address evolutionary aspects of the sdB stars themselves and, to investigate the role of planets in their formation, search for substellar companions to those. The method that will be applied to do so, is based on a pulsating subgroup of sdB stars, the variable subdwarf B stars (sdBV). As a synergy between asteroseismological methods and exoplanetary detection method, stellar pulsations will be a tool used in this thesis to search for (evolved) exoplanets or substellar objects in general.

The present thesis is an attempt to combine the two topics, evolution of subdwarf B stars and the presence of substellar companions, by applying a timing method to search for exoplanets around two particular target stars. The interplay between a host star and its substellar companion will be a central aspect in investigating their mutual influence and to provide a connection to evolutionary aspects. 


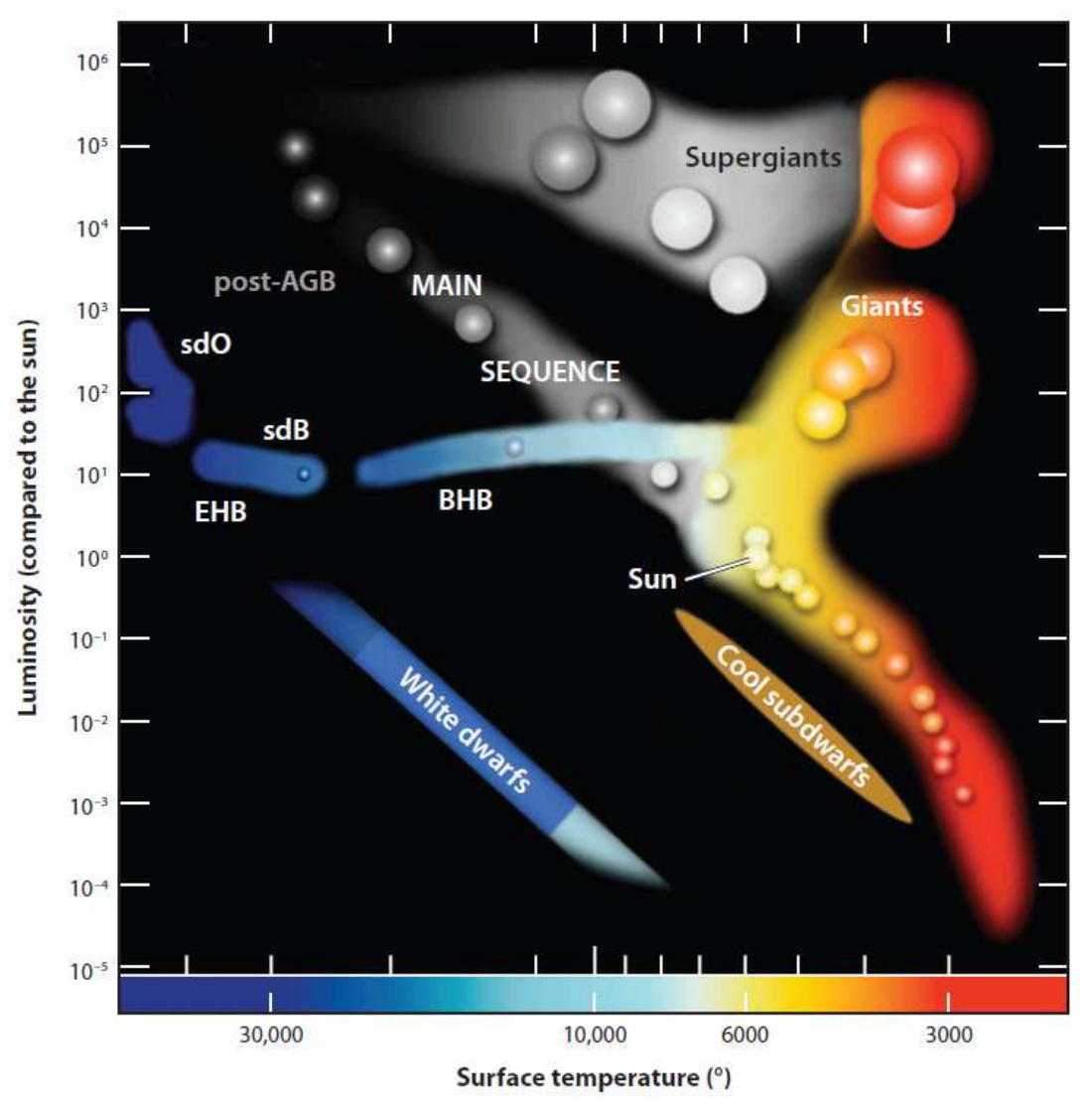

Figure 1.1: Hertzsprung Russell Diagram. From Heber (2009).

In the first section I will briefly review stellar evolution of low mass stars and focus particularly on subdwarf B stars in another section. Further I will expand on the basic concepts of asteroseismology and specifically on variable sdB stars (sdBV). Finally I will present a section on exoplanets, reviewing physical properties and putting them in a statistical context, with a focus set on evolved planetary systems.

\subsection{Stellar evolution}

To put the sdB stars considered in the present thesis in the context of the "typical" evolution of a low-mass star and to point out the sdB's special role and differences from it, I will first sketch the "typical" low-mass star evolution.

According to the Vogt-Russell theorem, a star's evolution and fate is mostly 
determined by its mass. Stars with masses below $8 M_{\odot}{ }^{1}$ will end their lives as a white dwarf. The evolution of a "typical" low-mass solar like star in the initial mass regime $0.4 M_{\odot}<\mathrm{M}<1.2 M_{\odot}$ is as follows: Once a cold dense cloud of interstellar matter, made of gas and dust particles, condenses due to gravitational instability, it will heat up according to the Virial theorem where half of the released potential energy is put into heating the cloud. Further condensation and fragmentation leads to the formation of protostars. The collapse stops when the core reaches hydrostatic equilibrium. Such an object (with a fully convective hydrostatic core of about a few hundred thousand Kelvin surrounded by a cool envelope of about 3000 Kelvin) is located on the Hayashi track. In a Hertzsprung-Russell diagram (HRD), the Hayashi track, which more precisely is a band of lines, marks the point where a star becomes fully convective. When the core reaches a temperature high enough to ignite hydrogen burning (at roughly ten million Kelvin) the object is defined to be a star and is located on the zero age main sequence. The star will then spend most of its lifetime burning hydrogen to helium on the main sequence (in the case of the sun for approximately ten billion years). After core hydrogen exhaustion, the star will leave the main sequence and burn hydrogen to helium in a shell surrounding the helium core. The contracting core heats up further and pushes the envelope outwards. This envelope expansion defines the red giant phase of evolution. At some point the helium core becomes electron degenerate and stops contracting but still continues to heat up. Due to a nearly temperature independence of the central pressure at that stage, the core temperature increase happens without a core expansion. Crossing the threshold temperature for helium burning, the rapid increase in temperature will lift degeneracy and make the core experience a sudden expansion, called the helium flash. The core mass at the helium flash is close to half a Solar mass. In the HRD, this event marks the tip of the red giant branch. During its red giant evolution the star may lose significant amounts of its mass. This effect and the immediate consequences for the star's further evolution in the special case of subdwarf B stars will be discussed thoroughly in the following section. After the core helium flash, the star settles down on the horizontal branch at lower luminosities and stably burns helium in the core accompanied by hydrogen

\footnotetext{
${ }^{1} 1 M_{\odot}=1.989 \cdot 10^{30} \mathrm{~kg}$
} 
burning in the surrounding shell. Some of the produced carbon may fuse with helium to oxygen to build up a carbon-oxygen core. For the low-mass stars considered here, after core helium exhaustion, the core temperatures will not be high enough to ignite stable carbon- or oxygen burning such that from now on the burning will only take place in the helium- and hydrogen burning surrounding shells. The star again becomes a giant, ascending the asymptotic giant branch. During this stage, the hydrogen and helium shells are consecutively active in a cyclic process called thermal pulses. Accompanied by a further mass loss the star will have expelled most of its envelope at the end of this stage. The shrinking carbon-oxygen core at constant luminosity together with the drastic increase in temperature will place the star in the upper left in the HRD. Once the previously ejected material gets ionized and lit up by the UV emission of the hot remnant core, the star has reached its planetary nebula phase. After this short phase, the remnant star (now having a mass close to about 0.6 solar masses) will slowly fade out as a white dwarf on the white dwarf cooling sequence. Now with the inward gravitational pressure balanced by the outward pressure of the core's degenerate electron gas, the white dwarf has no more energy sources left. Being such a hot (several ten thousand Kelvin) dense (about one million gram per centimeter cubed) and small (about an Earth's radius) object, resulting in a very small surface, it takes billions of years to radiate away the stored thermal energy. This star has now reached its final evolutionary stage. The location of the various stages mentioned in this short and highly simplified outline of low-mass stellar evolution can be recapitulated in the HRD shown in Figure 1.1.

\subsection{SdB stars}

Subdwarf B stars, however, do not follow this "typical" evolution described above. They represent a late evolutionary stage of low-mass stars. First discovered in a faint blue star survey by Humason \& Zwicky (1947) their position in the HRD (see Fig.1.1) was later determined bv Greenstein \& Sargent (1974). Further, Heber (1986) could connect the spectroscopic sdB class with the extreme horizontal branch (EHB) evolutionary stage. In a color-magnitude diagram (e.g. Fig.1.2) the horizontal branch, where the 


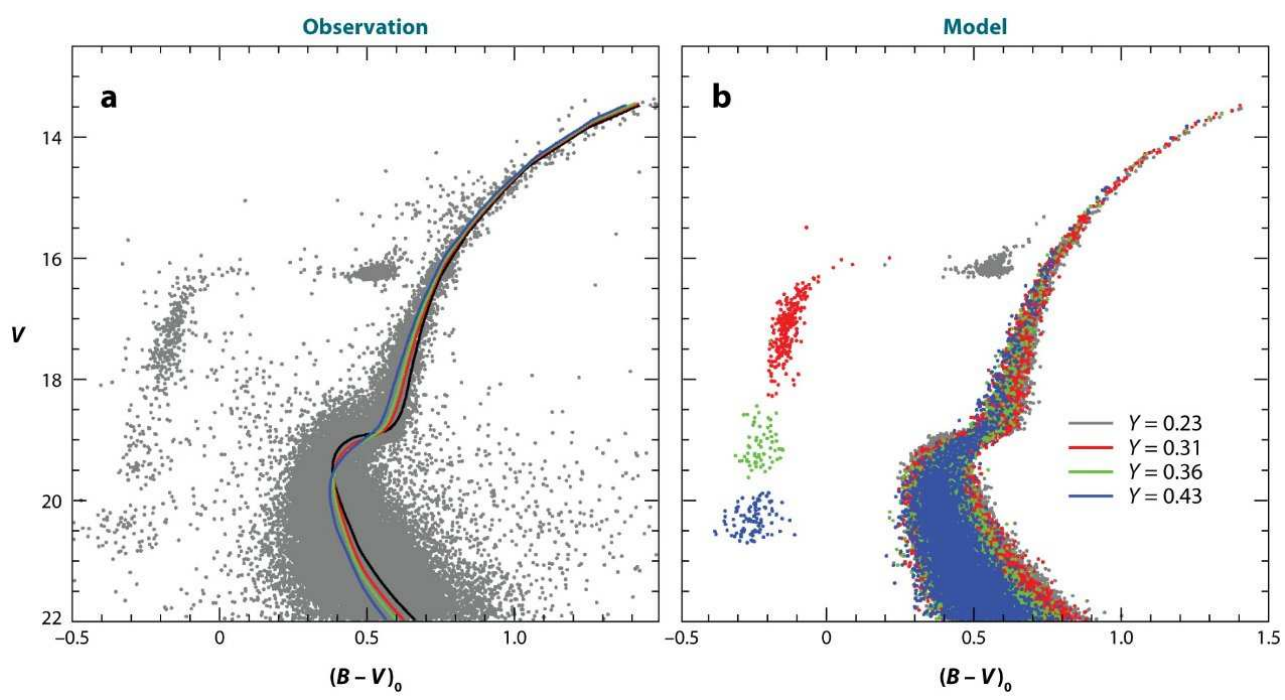

Figure 1.2: Color-magnitude diagrams of globular cluster NGC 2808. The horizontal branch is located at the left hand side of the plots. From Heber (2009).

stars are burning helium in their core, can clearly be distinguished from the hydrogen burning main sequence phase. The EHB is an extension of the horizontal branch towards the blue, i.e. towards higher temperatures (again, see Fig.1.1). SdB structure differs from the normal horizontal branch stars: the position on the horizontal branch depends on the mass loss during the RGB phase and the thickness of the remaining hydrogen envelope. Stars with thicker envelopes will be found at the cold end and stars with thinner envelopes on the hot end of the HB. However, some mechanism during the sdB progenitors giant phase removes that much of the envelope that after the helium flash the hydrogen envelope is too thin to sustain a stable hydrogen burning. This places these stars on the EHB and from there the evolution is no longer the "typical" one outlined above. The sdBs do not undergo thermal pulses (because of the inert hydrogen envelope), do not ascend the asymptotic giant branch and do not produce planetary nebulae (see Dorman et al., 1993, for post-HB evolutionary scenarios). Instead they directly evolve to the white dwarf cooling sequence. The reason for the sdBs' departure from the "typical" path is some mass loss mechanism which manages to remove all but a tiny fraction of the progenitor's hydrogen envelope at the time when the core reached the mass required for the helium 


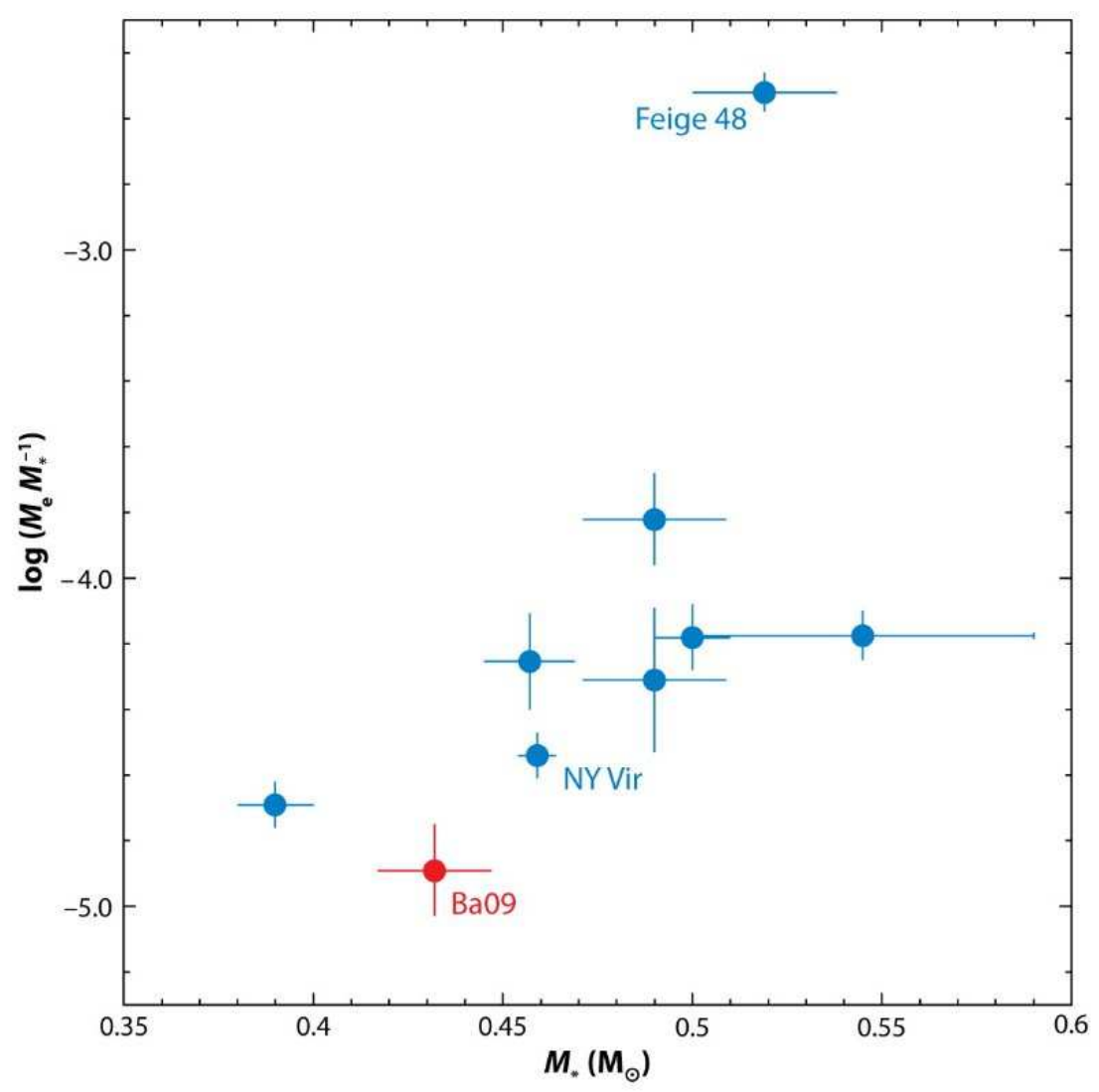

Figure 1.3: Asteroseismological masses of nine pulsating sdB stars versus fractional hydrogen envelope mass. The masses agree very well with the canonical sdB mass defined by the onset of the core helium flash at the RGB tip, which is restricted to the range 0.46-0.5 $M_{\odot}$. From Heber (2009).

flash, which is $\approx 0.5 M_{\odot}{ }^{2}$. There are some processes proposed to explain the required mass loss. I will recapitulate these in the following section. Spectroscopically, sdBs form a homogeneous class. They are found at effective temperatures roughly between $20000 \mathrm{~K}$ and $50000 \mathrm{~K}$ and surface gravities $\log g\left[g\right.$ in $\left.\mathrm{cm} \cdot \mathrm{s}^{-2}\right]$ between 5.0 and 6.5. Due to atomic diffusion, their abundances are peculiar. The atmosphere is dominated by hydrogen (broad Balmer lines) while helium is usually depleted (weak He I lines). As can be seen in Figure 1.4, light elements are also depleted (except of $\mathrm{N}$ ) while iron group elements are more or less strongly enriched (except of iron itself which is close to the Solar value) and lead is generally strongly overabundant. The iron group elements are of particular interest when considering the driving

\footnotetext{
${ }^{2}$ Therefore the canonical sdB mass is often declared to be roughly half a solar mass. Recent achievements include the asteroseismological mass determination of a sample of pulsating sdB stars, see Figure 1.3 .
} 


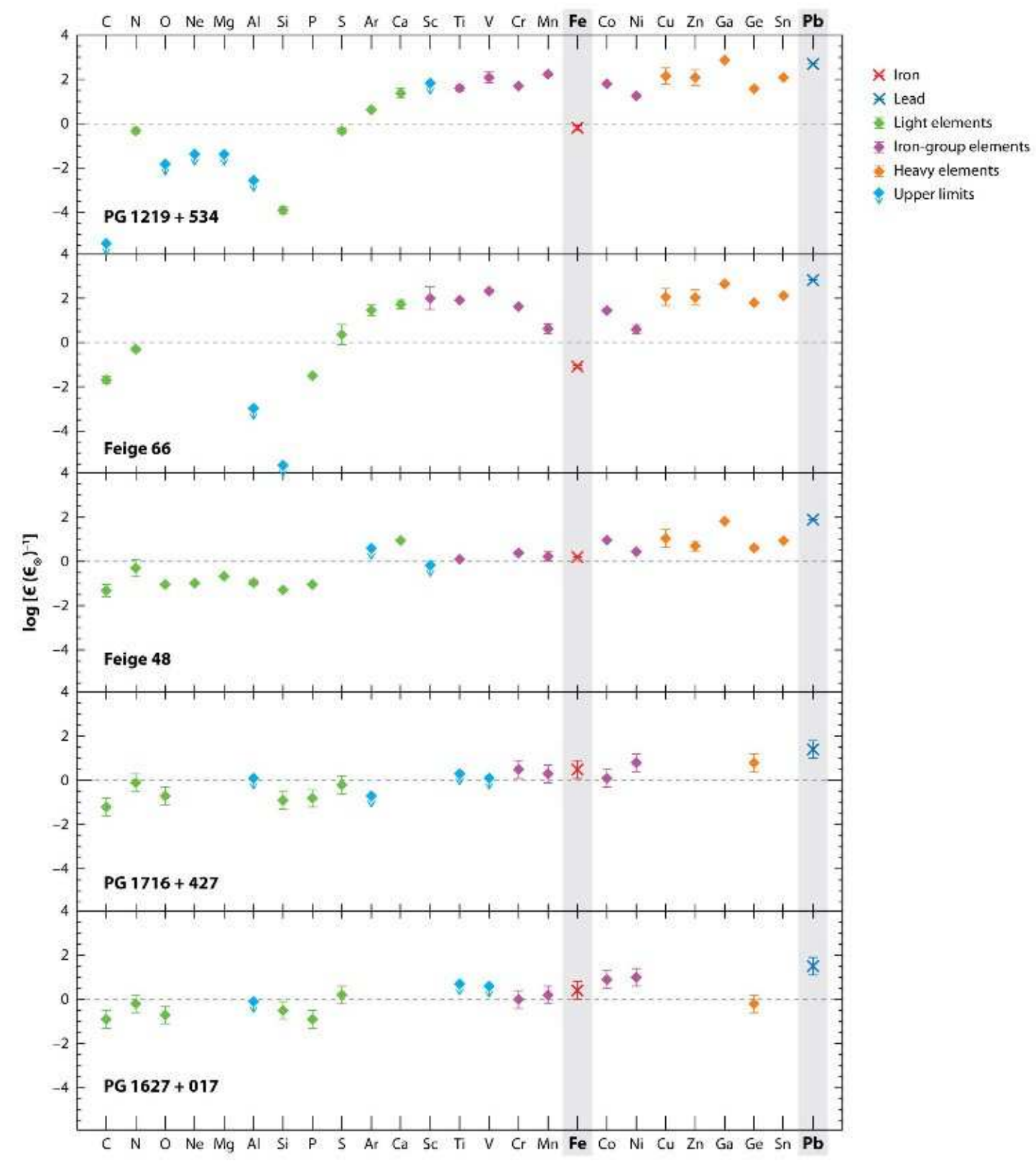

Figure 1.4: Abundances for five sdB stars. The temperature of the objects decreases from top $(33500 \mathrm{~K})$ to bottom $(22800 \mathrm{~K})$. From Heber (2009).

mechanism for pulsating sdB stars. I will come back to this later. The observed abundance patterns in sdBs can be explained by diffusive processes with a balance between gravitational settling and radiative levitation only if mass loss and turbulence is invoked too.

If not spun up in close binaries, spectra suggest slow rotational velocities of sdB stars below $10 \mathrm{~km} / \mathrm{s}$ (Geier et al., 2010, 2009b). 


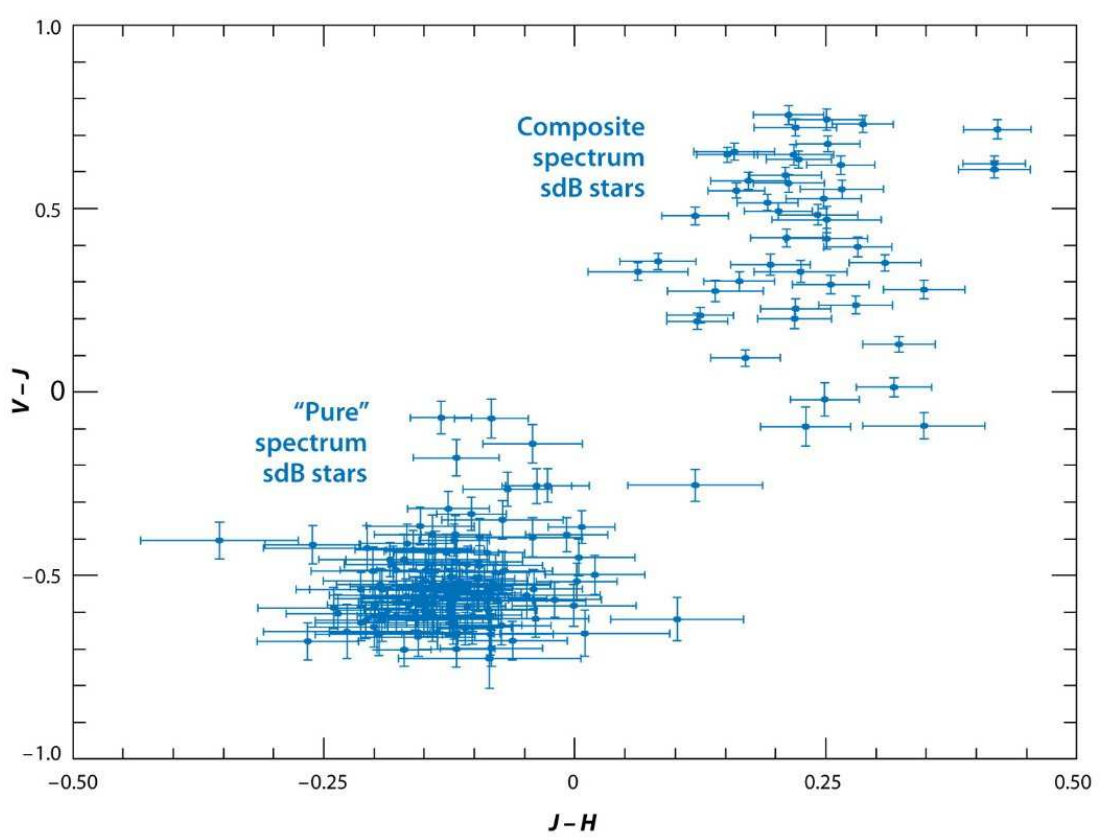

Figure 1.5: Two-color plot of sdB stars from the Green et al. (2008) sample. The upper right group is comprised of composite-spectrum sdBs while stars with pure sdB spectra are in the lower left. Apparently single sdB stars as well as binaries with invisible companions, either white dwarfs or M-dwarf secondaries, will fall into the lower left group. Plot from Heber (2009).

Heber (2009) gives an excellent review on hot subdwarf stars and summarizes the current status of sdB research. According to the subdwarf database at http://catserver.ing.iac.es/sddb/ (Østensen, 2006), the number of known subdwarf B stars is at least 1587.

\subsubsection{Formation and evolution}

After mentioning some general properties of sdB stars in the previous section, I will now put a focus on the formation and evolution of these objects. As already pointed out before, to explain sdB stars some mechanism must remove a significant fraction of the progenitor's envelope during the red giant evolution prior to the core helium burning. What kind of physical mechanism causes this mass loss is not very well understood but there are some formation scenarios proposed for binary- as well as single star sdB evolution. I will outline different formation channels which apply either to sdB stars in binaries or apparently single sdB stars after presenting the results of some sample studies. 
A distribution of composite-spectrum versus pure spectrum sdBs from a given sample is depicted in Figure 1.5. In another study, analyzing $2 \mathrm{MASS}^{3}$ sdB colors, Stark \& Wade (2003) find 30-40\% (in a volume-limited and a magnitude-limited sample, respectively, consisting of several hundred hot subdwarfs) to be consistent with the presence of an unresolved late-type main-sequence companion. Radial velocity surveys revealed that many apparently single sdB stars do actually reside in close binary systems (periods less than ten days) with an invisible companion, either being a low-mass main-sequence star or a white dwarf. The former could also be revealed by a reflection effect in the light curves while the latter would be revealed by ellipsoidal deformation. The fraction of these close binaries in two particular samples is found to be $66 \%$ and $40 \%$ (Maxted et al.. 2001: Napiwotzki et al., 2004).

Concerning the binary sdBs, scenarios including mass transfer and common envelope (CE) evolution seems to be most promising to explain the binary sdB population.

Recent studies (Han et al., 2002, 2003; Podsiadlowski et al., 2008) distinguish three channels of close-binary evolution (shown in Figure 1.6) yielding three different final configurations. Depending on the initial mass ratios, these channels are: (i) stable Roche-lobe overflow (RLOF) plus common envelope (CE), (ii) CE only and (iii) stable RLOF only. These are sketched in the panels a, b and $\mathrm{c}$ in Figure 1.6. Let us assume the sdB progenitor to be a giant star on the RGB filling its Roche lobe. For mass ratios of the giant donor to the MS acceptor bigger than 1.2-1.5, mass transfer is believed to be dynamical (unstable RLOF). As the acceptor will not be able to accrete all matter, it will fill its own Roche lobe too and a common envelope surrounding both components will build up. Friction with this CE will cause the components to spiral towards each other until enough energy is released to eject the envelope and leave behind a close binary consisting of the giant core which will become the sdB and the MS component. This channel is sketched in panel b of Figure 1.6 and results in a short-period sdB+MS binary. In contrast, for mass ratios of the giant donor to the MS acceptor smaller than 1.2-1.5, mass transfer is generally believed to be stable, such

${ }^{3}$ http://www.ipac.caltech.edu/2mass/ 
a

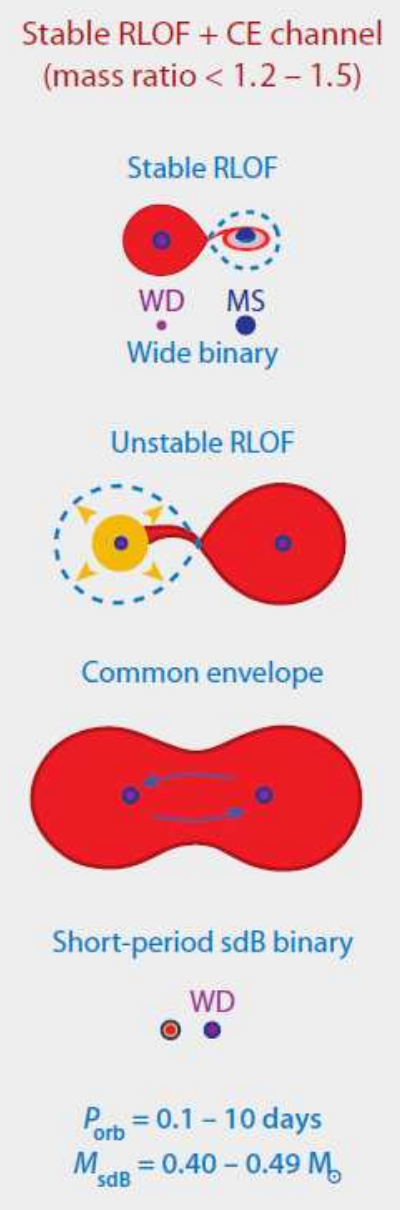

b

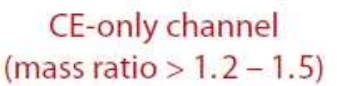

(mass ratio $>1.2-1.5$ ) c

Stable RLOF channel

(mass ratio $<1.2-1.5$ )

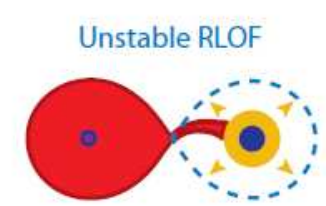

Common envelope

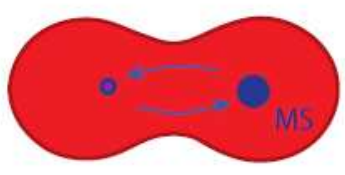

Short-period sdB binary

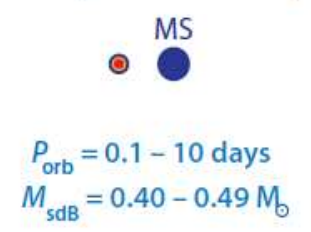

sdB with MS/SG companion

- Wide binary

$P_{\text {orb }}=10-500$ days

$M_{\text {sdB }}=0.30-0.45 \mathrm{M}$

Figure 1.6: Formation scenarios for binary sdBs. The temporal evolution proceeds from top to bottom. The final configurations of the three channels $\mathrm{a}, \mathrm{b}$ and $\mathrm{c}$ are short-period $\mathrm{sdB}+\mathrm{WD}$ or sdB+MS binaries or wide long-period binaries with MS or subgiant $(\mathrm{SG})$ companions. See text for further information. Image taken from Heber (2009). Originally based on Podsiadlowski (2008).

that the acceptor can accrete all the transferred matter. This in combination with the absence of a $\mathrm{CE}$ will tend to increase the orbital separations. If this stable RLOF happens close to the RGB tip, it will produce wide sdB + MS binaries (see panel c in Figure 1.6). However, it may become more complicated if there are more phases of mass transfer. For example, if the first mass transfer phase is stable (but insufficient to produce a sdB star) it may form a wide WD+MS binary. In a second mass transfer phase the MS component (which will later be the sdB) may then evolve into a giant and 
trigger an unstable RLOF by filling its Roche lobe. The resulting common envelope will then, due to friction and spiral-in effects, form a short-period binary. Only this time it consists of sdB+WD components. This scenario is shown in panel a of Figure 1.6. Further considerations concerning binary evolution can be found in Podsiadlowski \& Han (2004).

Still, one has to keep in mind that a detailed physical description of CE evolution has not yet emerged. Hu et al. (2007) present two different formalisms: the standard $\alpha$-formalism based on the energy equation and an alternative description (the $\gamma$-formalism) based on the angular momentum equation. A recent overview is given by Ivanova (2011). Observational data are so far insufficient to put tight constraints on either of the formalisms.

I will now focus on single sdB stars and present the proposed formation channels. The necessary enhancement in mass loss near the RGB tip may be due to strong stellar winds or internal rotation (top panel in Figure 1.7), but very little is known about these effects which are therefore hard to comment upon. In general, the whole mass loss process itself and the underlying physical mechanisms are barely understood.

Another proposed channel for forming single sdB stars is a merger scenario (bottom panel in Figure 1.7) of two helium white dwarfs which lose orbital energy through gravitational radiation and therefore spiral towards each other. The less massive component could then be disrupted and accreted onto the remaining component which may ignite helium after exceeding a critical mass and so become an sdB star. Since the merger product is believed to be enriched in CNO (Saio \& Jeffery, 2000), this is in contradiction with the abundance patterns found for sdB stars (see Fig. 1.4). Another argument against the WD-merger scenario is that the merger product would rotate faster than the breakup velocity if conservation of angular momentum is assumed (Gourgouliatos \& Jeffery, 2006).

It could also be possible that during the CE phase in a binary channel (as sketched above) a low-mass star merges with the primary red giant. The merger product would be a rapidly rotating HB star with centrifugally enhanced mass loss. This common envelope merger scenario is investigated in Politano et al. (2008) and it is suggested that, due to the enhanced mass loss, some of these common envelope mergers may lead to the formation of single sdB stars. Clausen \& Wade (2011) describe a scenario where the 


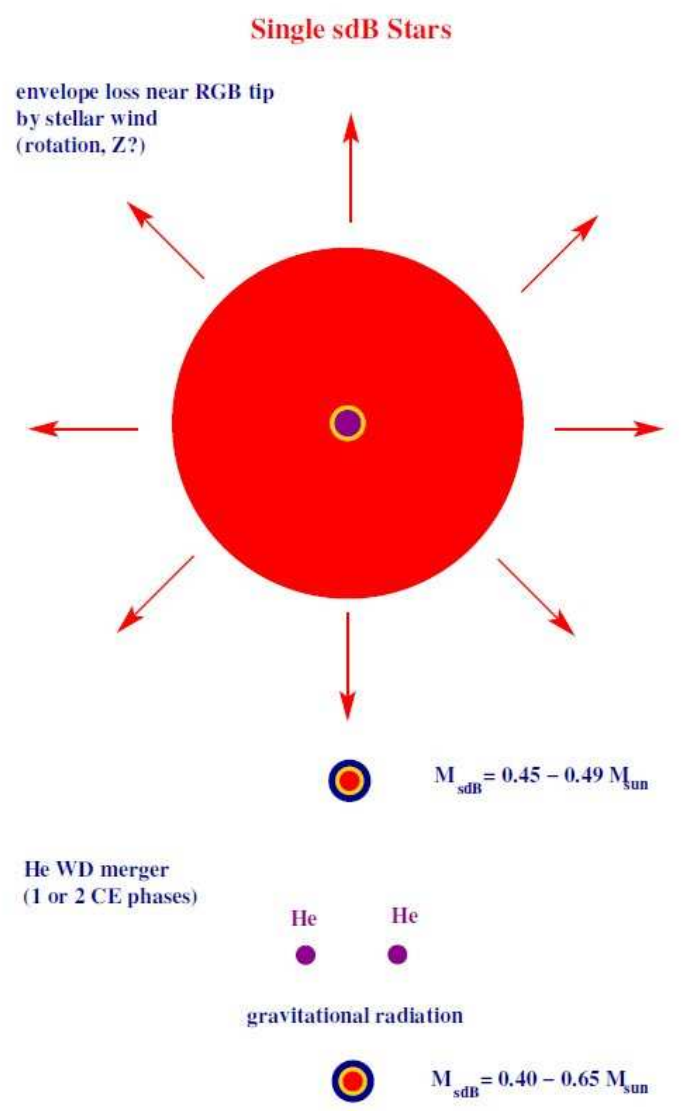

Figure 1.7: Formation scenarios for single sdBs. Image taken from Podsiadlowski (2008).

merger product of a helium white dwarf and a low mass star under certain conditions can possibly evolve to become a single sdB star.

A further scenario proposed to explain single sdB stars includes so-called hot flashers (see Fig. 1.8). While typically the helium flash occurs at the tip of the RGB (panel a), it is also possible that, provided a sufficient mass loss, the star can depart from the RGB and the helium core flash takes place later (panels b, c and d). These stars are then called hot flashers. The remnants of these hot flashers lie close to the helium-main sequence ${ }^{4}$, which roughly coincides with the sdB location. Although an 'early hot flasher' scenario may explain some abundance patterns in sdBs, it is not consistent with observations in detail. For a closer look on the different hot flasher

\footnotetext{
${ }^{4}$ The helium main sequence (He-MS) is the position in the HRD where a star which has no envelope left at the time of the RGB tip core helium flash would be placed. The He-MS and the very hot end on the horizontal branch, i.e. the EHB, overlap in the $\log g-T_{\text {eff }}$ diagram.
} 


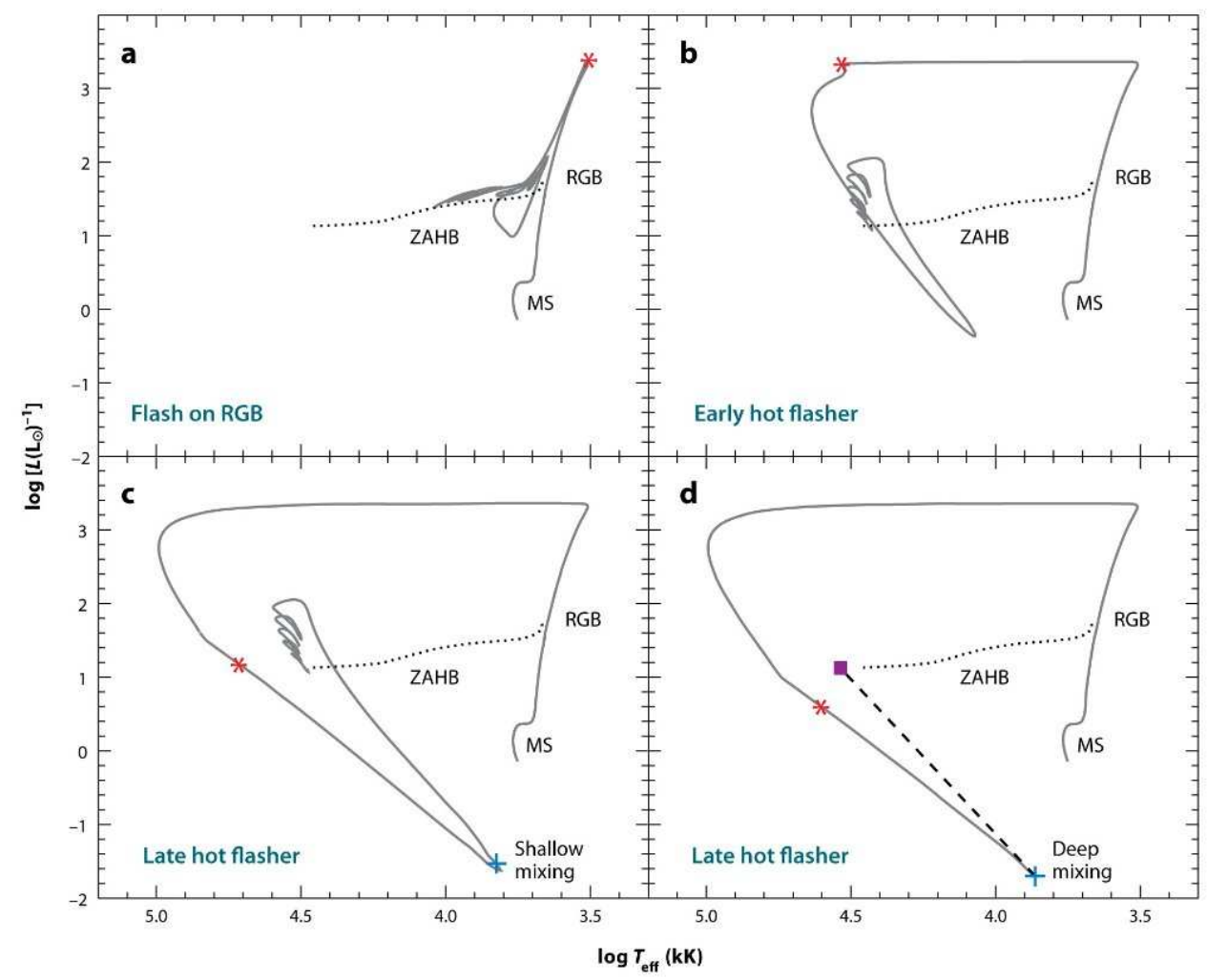

Figure 1.8: Hot flasher scenarios. The position of the core helium flash is indicated by an asterisk. The dotted line marks the zero age horizontal branch. For further information see Heber (2009) and D'Cruz et al. (1996).

scenarios shown in Fig. 1.8, I refer to Heber (2009) and references therein.

Finally I want to mention a formation channel that was proposed already in the late 1990s and received special attention recently due to the discovery of the first exoplanet found to have survived the red giant phase of its host star (Silvotti et al., 2007):

As shown by a study of Soker (1998), substellar objects like brown dwarfs and even companions as small as exoplanets will be able to provide an enhanced mass loss sufficient to form a single sdB star. In analogy to the binary evolution channels introduced earlier (where both components are stellar), this scenario predicts that substellar objects are able to influence the evolution of their host stars.

A similar study of the influence of substellar companions on their host stars in the case of white dwarfs is given by Nelemans \& Tauris (1998). In addition, Maxted et al. (2006) provide evidence that substellar objects may not 
only be able to trigger enhanced mass loss but also that they can survive engulfment in the red giant's envelope.

Bear \& Soker (2011b) and Geier et al. (2011a) provide up-to-date studies on exoplanets around extreme horizontal branch stars, both theoretically and observationally.

\subsection{Asteroseismology}

In the last section I introduced general properties and characteristics of subdwarf B stars. The method applied in this thesis to search for exoplanets can only be applied to a pulsating subgroup of the sdB stars, the subdwarf B variables (sdBV). Before introducing these in more detail in a later section, I will now first expand on pulsations in stars in general and summarize the basic concepts of asteroseismology and the driving mechanism behind those pulsations relevant for our study.

\subsubsection{Stellar pulsations}

The basic concept of non-radial stellar pulsations is as follows: Using the equations of hydrodynamics, one can derive the oscillation properties by perturbing the equilibrium quantities.

Kurtz (2005) gives a nice overview on asteroseismology in general with some observational aspects. A very detailed theoretical description of asteroseismology and the current status of various applications to different types of pulsating stars is given in the text book by Aerts et al. (2010), who also address aspects of observational techniques and frequency analysis. Essential parts of the present subsection on the theoretical basics of stellar pulsations closely follow the diploma thesis of Lutz (2007) and references therein.

Adiabatic approximations and the mechanism, which is believed to be responsible for the pulsations in sdB stars can be found at the end of this section.

The simplest way for a star to oscillate is via radial oscillations, where a star contracts and expands only radially and therefore retains spherical symmetry during the whole cycle. Radial oscillations can be characterized by the 
radial order $k$. This wave number describes the number of nodes between the center and the surface of the star. Well-known radial oscillators are Cepheids, or RR Lyrae stars.

The evolved EHB stars that are dealt with in this thesis are non-radial oscillators. This means that transverse motions occur in addition to the radial ones. To take this into account, two more wave numbers are necessary: the degree $l$ and the azimuthal order $m$, which appear in the spherical harmonics $Y_{l}^{m}(\theta, \phi)$, representing the dependence of the mode on the latitude and longitude angles $\theta$ and $\phi$. I will come back to spherical harmonics later.

\section{Basic equations of hydrodynamics}

Let us first consider the general equations of hydrodynamics. In the following, we assume that a star can be approximated as a spherically symmetric gaseous sphere. Additionally, effects like magnetic fields and rotation are neglected.

There exist two formalisms to describe the hydrodynamics of a star: the Lagrangian and the Eulerian description. The Lagrangian description is that of an observer who follows the motion of a gas element. In contrast to this, the Eulerian description is that of a stationary observer such that the properties of a gas element can be specified as functions of the current position and time. The time derivative of any quantity $X$ following the movement of a mass element will be denoted by the total derivative $d X / d t$, i.e. Lagrangian. The time derivative in the Eulerian description will be denoted as the partial derivative $\partial X / \partial t$ and is valid for a given fixed position in space. The time derivatives of a quantity $X$ for the both formalisms are connected via

$$
\frac{d X}{d t}=\frac{\partial X}{\partial t}+\vec{v} \cdot \nabla X
$$

where $\vec{v}$ denotes the local instantaneous velocity. $d / d t$ is also known as the material time derivative whereas $\partial / \partial t$ is known as the local time derivative (i.e., the time derivative at a fixed point).

The following equations form a complete set of equations of hydrodynamics: 
1. The equation of mass conservation (continuity equation):

$$
\frac{d \rho}{d t}+\rho \nabla \cdot \vec{v}=\frac{\partial \rho}{\partial t}+\nabla \cdot(\rho \vec{v})=0,
$$

where $\vec{v}$ and $\rho$ are the local velocity and density.

2. The equation of momentum conservation (equation of motion):

$$
\frac{d \vec{v}}{d t}=\frac{\partial \vec{v}}{\partial t}+\vec{v} \cdot \nabla \vec{v}=-\nabla \psi-\frac{1}{\rho} \nabla p,
$$

where $p$ is the total pressure (gas, radiation and turbulent pressure) and $\psi$ is the gravitational potential which fulfills the Poisson equation:

$$
\Delta \psi=4 \pi G \rho .
$$

The gradient of the gravitational potential is the gravitational acceleration $\vec{g}$ :

$$
\vec{g}=-\nabla \psi
$$

3. The equation of energy conservation:

$$
T \frac{d S}{d t}=\varepsilon-\frac{1}{\rho} \nabla \cdot \vec{F}
$$

where $T$ is the local temperature, $S$ the entropy, $\varepsilon$ the rate of nuclear energy generation and $\vec{F}$ the energy flux.

If one only considers radiative flux for energy transport and applies a diffusion approximation, the radiative flux $\overrightarrow{F_{R}}$ is given by the diffusion equation:

$$
\overrightarrow{F_{R}}=-\frac{4 a c T^{3}}{3 \kappa \rho} \nabla T
$$

with the radiation density constant $a$, the speed of light $c$ and the Rosseland mean opacity $\kappa$, which is defined by

$$
\frac{1}{\kappa}=\frac{\int_{0}^{\infty} d \nu \frac{1}{k_{\nu}} \frac{d B_{\nu}}{d T}}{\int_{0}^{\infty} d \nu \frac{d B_{\nu}}{d T}} .
$$

$\frac{k_{\nu}}{\rho}$ is the specific opacity and $B_{\nu}$ denotes the Planck-function

$$
B_{\nu}(T)=\frac{2 h \nu^{3}}{c^{2}} \frac{1}{\exp \left(\frac{h \nu}{k T}\right)-1} .
$$




\section{Equilibrium structure and perturbation analysis}

A general hydrodynamical description of a star is far too complex to handle numerically. In the case of stellar oscillations one can fortunately apply some simplifications, because most of the energy in the motion is in regions with relatively small amplitudes (at least in the kind of pulsators we are looking at in this thesis). Thus, the properties of the oscillations can be understood in terms of small perturbations around a static equilibrium state.

We now consider a static equilibrium structure, such that all time derivatives and velocities can be neglected.

Given this, the continuity equation Eq.(1.2) is trivially satisfied and the equation of motion Eq.(1.3) reduces to the equation of hydrostatic support,

$$
\nabla p_{0}=\rho_{0} \vec{g}_{0}=-\rho_{0} \nabla \psi_{0} .
$$

The equilibrium quantities are denoted with the subscript "0". Poisson's equation Eq.(1.4) in equilibrium is

$$
\Delta \psi_{0}=4 \pi G \rho_{0},
$$

and the energy equation Eq.(1.5) simplifies to

$$
0=\epsilon_{0}-\frac{1}{\rho_{0}} \nabla \cdot \vec{F}_{0} .
$$

Assuming spherical symmetry, the structure only depends on the distance $r$ to the center. Then $\vec{g}_{0}=-g_{0} \vec{e}_{r}$, where $\vec{e}_{r}$ is a unit vector directing radially outwards.

With this, Eq.(1.9) becomes the equation of hydrostatic equilibrium, which is

$$
\frac{d p_{0}}{d r}=-g_{0} \rho_{0} .
$$

Further, one can specify $g_{0}$ at position $r$ by integrating Poisson's equation 
once, which yields

$$
g_{0}=\frac{G}{r^{2}} \int_{0}^{r} 4 \pi \rho_{0} r^{\prime 2} d r^{\prime}=\frac{G m_{0}}{r^{2}} \quad \Rightarrow \quad \frac{d m_{0}}{d r}=4 \pi r^{2} \rho_{0}
$$

where $m_{0}(r)$ denotes the mass inside the sphere with radius $r$.

With the flux $\vec{F}_{0}=F_{r, 0} \vec{e}_{r}$ directed radially outwards, the energy equation Eq.(1.11) can be rearranged to

$$
\rho_{0} \epsilon_{0}=\frac{1}{r^{2}} \frac{d}{d r}\left(r^{2} F_{r, 0}\right)=\frac{1}{4 \pi r^{2}} \frac{d L_{0}}{d r},
$$

with $L_{0}=4 \pi r^{2} F_{r, 0}$ being the luminosity or the total flow of energy through the sphere with radius $\mathrm{r}$. This gives us

$$
\frac{d L_{0}}{d r}=4 \pi r^{2} \rho_{0} \epsilon_{0}
$$

Finally the diffusion equation Eq.(1.6) for the radiative flux can be written as

$$
\frac{d T_{0}}{d r}=-\frac{3 \kappa_{0} \rho_{0}}{16 \pi r^{2} a c T_{0}^{3}} L_{0}
$$

Equations (1.12) to (1.15) provide the set of equations for equilibrium stellar structure.

However, at a given time a pulsating star is not in equilibrium but the characteristics of a mass element (position, density, pressure, temperature) vary around their equilibrium values. Considering small perturbations around the equilibrium state, a quantity $\mathrm{X}$ may be written as $X(\vec{r}, t)=X_{0}(\vec{r})+$ $X^{\prime}(\vec{r}, t)$, where $X^{\prime}$ is a small perturbation at a given point. Equations for the perturbations can then be obtained by linearization, which is inserting the above expression in the full equations, subtracting the equilibrium equations and neglecting quantities of order higher than one in $X^{\prime}$. I will not expand on this further at this point but refer to Aerts et al. (2010) for a 
detailed description.

Let us now have a closer look on the description of a pulsational mode. As mentioned before, a non-radial mode ${ }^{5}$ can be characterized by three wave numbers: the degree $l$ and the azimuthal order $m$ determine the behavior of the mode on the surface and the radial order $k$ denotes the properties in the radial direction. In a descriptive way, one can regard $l$ as the total number of node lines on the star's surface and $m$ as the number of lines which cross the equator (i.e. the number of longitude lines).

A general displacement $\overrightarrow{\delta r}$ can be separated into a radial component and a horizontal component: $\overrightarrow{\delta r}=\xi_{r} \vec{e}_{r}+\vec{\xi}_{h}$. After a lot of mathematics, which is beyond the scope of this chapter but entirely treated in chapter 3.3 of Aerts et al. (2010), one can find solutions for both components and finally describe a nonradial oscillation by

$$
\begin{aligned}
& \overrightarrow{\delta r}(r, \theta, \phi)=\sqrt{4 \pi} . \\
& \operatorname{Re}\left\{\left[\xi_{r}(r) Y_{l}^{m}(\theta, \phi) \vec{e}_{r}+\xi_{h}(r)\left(\frac{\partial Y_{l}^{m}}{\partial \theta} \vec{e}_{\theta}+\frac{1}{\sin \theta} \frac{\partial Y_{l}^{m}}{\partial \phi} \vec{e}_{\phi}\right)\right] \exp (-i \omega t)\right\} .
\end{aligned}
$$

The angular dependence is described by spherical harmonics $Y_{l}^{m}(\theta, \phi)$, where $\theta$ is the latitude (angular distance from the polar axis) and $\phi$ is the longitude:

$$
Y_{l}^{m}(\theta, \phi)=(-1)^{m} c_{l m} P_{l}^{m}(\cos \theta) \exp (i m \phi) .
$$

Here $P_{l}^{m}$ is a Legendre function and $c_{l}^{m}$ is a normalization constant.

Since general oscillation equations can be quite complicated, we want to apply further approximations. Neglecting the perturbation of the gravitational potential ( $\psi^{\prime}=0$, known as the Cowling approximation) is valid for large degrees $l$ and large radial orders $k$ (the latter is also prerequisite for an asymptotic theory). Further neglecting derivatives of equilibrium quantities as well as considering an adiabatic approximation (i.e. neglecting the

\footnotetext{
${ }^{5}$ Radial oscillations are a special case of non-radial modes and have the degree $l=0$.
} 
heating term in the energy equation), the Cowling approximation results in a single second-order differential equation for $\xi_{r}$ :

$$
\frac{d^{2} \xi_{r}}{d r^{2}}=-K(r) \xi_{r}
$$

with

$$
K(r)=\frac{\omega^{2}}{c^{2}}\left(\frac{N^{2}}{\omega^{2}}-1\right)\left(\frac{S_{l}^{2}}{\omega^{2}}-1\right) .
$$

Equation 1.18 is the crudest possible approximation for nonradial oscillations. In equation 1.19, $c$ is the adiabatic sound speed, $S_{l}$ denotes the characteristic sound frequency, or Lamb-Frequency, and $N$ denotes the buoyancy frequency or Brunt-Väisälä-Frequency:

$$
S_{l}^{2}=\frac{l(l+1) c^{2}}{r^{2}} \quad \text { and } \quad N^{2}=g\left(\frac{1}{\Gamma_{1} p} \frac{d p}{d r}-\frac{1}{\rho} \frac{d \rho}{d r}\right)
$$

with $g=\frac{G m}{r^{2}}$ and $\Gamma_{1}=\left(\frac{\partial \ln p}{\partial \ln \rho}\right)_{a d}$.

For $K>0$ the solution $\xi_{r}$ is locally an oscillating function of $r$ while it is exponentially decreasing or increasing for $K<0$. Thus the solution oscillates if

$$
|\omega|>|N| \quad \text { and } \quad|\omega|>S_{l},
$$

or

$$
|\omega|<|N| \quad \text { and } \quad|\omega|<S_{l} .
$$

Modes with high frequencies satisfying Eq.(1.21) are labeled pressure modes or $p$-modes, whereas modes with low frequencies satisfying Eq.(1.22) are labeled gravity modes or g-modes. The regions within a star where Eq.(1.21) and Eq.(1.22) are satisfied (and thus the modes have an oscillatory behavior) are called the trapping regions or mode cavities. The g-mode cavities 
are typically situated much deeper in the star than the p-mode cavities. Generally one can say that waves with small degree $l$ are deeply penetrating, while those with higher degree $l$ are shallowly penetrating. Highlighting some differences between $p$ - and $g$-modes, one can note that $p$-modes attain large amplitudes in the outer layers and $g$-modes in the deeper layers. For $p$-modes the frequency increases with the mode order whereas $g$-mode frequencies decrease with the mode order. I will address further differences between $p$ - and $g$-modes in the particular case of the sdBV stars later.

\section{Driving mechanism}

For reasons of brevity only the opacity mechanism or $\kappa$-mechanism, which is thought to be responsible for pulsations in sdB stars, will be discussed in this section. Further details can be found in Aerts et al. (2010). The $\kappa$-mechanism with respect to sdB pulsators is addressed in Charpinet et al. (1997), Fontaine et al. (2003) and Charpinet (2001) or Jeffery \& Saio (2006a) and Jeffery \& Saio (2006b).

The main effect that determines the driving of a mode results from a perturbation of the opacity $\kappa$ which results in a perturbation of the luminosity $L$. Regions where $\delta L$ is decreasing outwards during a hot phase $\delta T>0$ have a driving effect, whereas regions with $\delta L$ increasing outwards during the hot phase have a damping effect. One can write the luminosity perturbation as

$$
\frac{\delta L}{L}=4 \frac{\delta r}{r}+\left(4-\kappa_{T}\right) \frac{\delta T}{T}-\kappa_{\rho} \frac{\delta \rho}{\rho}-\left(\frac{d \ln T}{d m}\right)^{-1} \frac{d}{d m}\left(\frac{\delta T}{T}\right),
$$

where $\kappa_{T}=(\partial \ln \kappa / \partial \ln T)_{\rho}$ and $\kappa_{\rho}=(\partial \ln \kappa / \partial \ln \rho)_{T}$. For low order modes one can neglect the last term as well as the first term of the right hand side. With $\delta T / T \simeq\left(\Gamma_{3}-1\right) \delta \rho / \rho$, which is valid where the oscillations are nearly adiabatic (the adiabatic exponent $\Gamma_{3}$ is defined by $\Gamma_{3}-1=(\partial \ln T / \partial \ln \rho)_{a d}$ and equals $5 / 3$ in an ideal gas), one has

$$
\frac{\delta L}{L} \simeq\left[\left(4-\kappa_{T}\right)\left(\Gamma_{3}-1\right)-\kappa_{\rho}\right] \frac{\delta \rho}{\rho},
$$

Since $\delta \rho / \rho$ generally increases outwards it is clear that one needs a strong decrease in $\Gamma_{3}$ or a strong increase in $\kappa_{T}$, such that the decrease in $\delta L$, which 
is necessary for driving, is achieved. Both effects are likely to occur in ionization zones of abundant elements. There is also a tendency that these ionization zones are associated with large opacity bumps: $\kappa_{T}$ can increase very steeply and therefore provide the necessary decrease in $\delta L$ during a hot phase. This perturbation of the opacity plays a dominant role in the driving of sdB pulsations and hence one speaks of the $\kappa$-mechanism. In sdBV stars the bump in the opacity profile is related to elements of the iron-group, which are accumulated in the driving zones by diffusion processes like gravitational settling and radiative levitation.

In a more descriptive way, one could say that a partial ionization layer is periodically excited/unexcited and acts as a heat engine. Such a layer can trap the energy radiated outwards by the core during a small contraction and release it during the subsequent expansion. Then one has the following simplified picture: a suitable layer compresses causing pressure and temperature to increase (hot phase). Then excitation of the participating elements increases the partial ionization and opacity increases. Hence, the radiation from deeper layers is trapped and the condition for driving as defined above is fulfilled ( $\delta L$ decreasing during a hot phase due to an increase of opacity). As a result, radiation pressure increases and the layer expands. The temperature gets cooler and recombination decreases the partial ionization, which causes the opacity to decrease such that the accumulated energy can be released. The pressure underneath the layer decreases again and the layer can compress again, leading to a cyclic process. The timing of this cyclic process is essential for its efficiency. Just like a swing which has to be pushed right after the minimum position if one wants to achieve a maximum efficiency and to avoid damping. The basic requirement for this mechanism to work is that the opacity increases during a compression (hot phase $\delta T>0$ ). In most of the star the opacity is proportional to $\rho / T^{3.5}$ (Kramers opacity) and would hence decrease with increasing temperature, unfavorable for driving. As schematically outlined above, the most likely cases, however, where the opacity increases during a hot phase and therefore favors driving, are the partial ionization layers with the associated opacity bumps.

It is necessary that these layers, where pulsations can be driven, are located at a suitable position in the star, i.e. at positions where one has the opacity bump, such that the $\kappa$-mechanism can work and make the star oscillate. 
That is why radial and non-radial oscillations are only excited for suitable combinations of stellar luminosity, temperature and chemical composition. The regions in the HRD where the conditions are given such that a star may be able to oscillate are the so called instability strips. Figure 1.9 shows various instability strips throughout the HRD with the corresponding pulsating stellar types.

\subsubsection{SdBV stars}

After reviewing the theoretical background of pulsations, I want to take a closer look at variable sdB stars. The asteroseismological modeling of sdBVs is described in Charpinet et al. (2007, 2009) while Fontaine et al. (2008) and Østensen (2009) provide general reviews on sdB asteroseismology. The most recent survey for pulsating sdB stars is presented in Østensen et al. (2010). Only a small fraction of the sdB stars is found to be variable. Providing a reliable fraction is, of course, dependent on the observational sensitivity. Given the sensitivity of ground-based surveys so far one could declare a star which does not exhibit variations above one mmag to be a non-pulsator. In that case the non-pulsators would outnumber the pulsators roughly by a factor of ten. If the fact that pulsators and non-pulsators can coexist in the same region of the $\log g-T_{\text {eff }}$ plane is just due to this observational bias against low amplitudes has still to be tested. Improvements are achieved with the Kepler spacecraft which also finds pulsations below one mmag since it is much more sensitive towards lower amplitudes than the ground-based telescopes.

Pulsations in subdwarf B stars were theoretically predicted and observationally found nearly at the same time (Charpinet et al.. 1996: Kilkenny et al., 1997). Today it is known that both types of pulsations, pressure driven $p$-modes and gravity driven $g$-modes (cf. equations 1.21 and 1.22) can appear in sdBV stars. The prototype p-mode pulsator EC 14026-2647 (or V361 Hya) was discovered by Kilkenny et al. (1997). Later, Green et al. (2003) discovered the $g$-mode prototype PG 1716+426 (or V1093 Her) and Schuh et al. (2006) the hybrid prototype HS 0702+6043 (or DW Lyn). For both mode types, the driving mechanism is believed to be the $\kappa$-mechanism (Charpinet et al., 1997; Fontaine et al., 2003).

To provide a uniform nomenclature of the different classes, Kilkenny et al. 


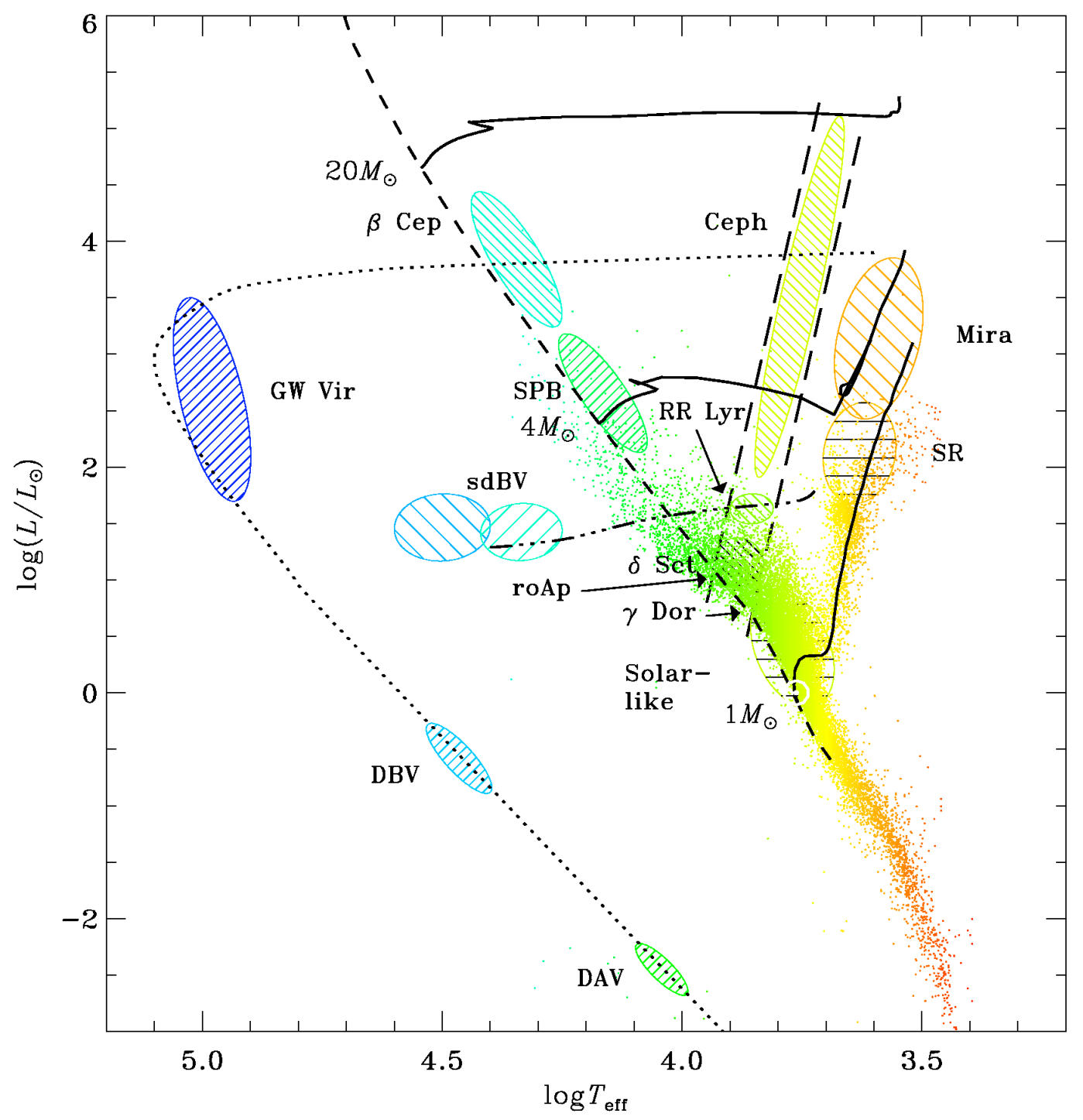

Figure 1.9: Pulsational HRD showing the location of the pulsating sdB stars (sdBV) on the extreme horizontal branch. The top-left to bottom-right hatching indicates $p$-mode pulsators, the bottom-left to top-right hatching indicates $g$-mode pulsators and the horizontal hatching identifies solar-like oscillations. See text for further details. This plot is a modified version from http://astro.phys.au.dk/HELAS/puls_HR.

(2010) proposed the following scheme that I will adopt throughout this thesis: $\mathrm{sdBV}_{r}$ for the rapid $p$-mode pulsators, $\mathrm{sdBV}_{s}$ for the slow $g$-mode pulsators and $\mathrm{sdBV}_{r s}$ for the hybrids exhibiting both types of modes at the same time. 


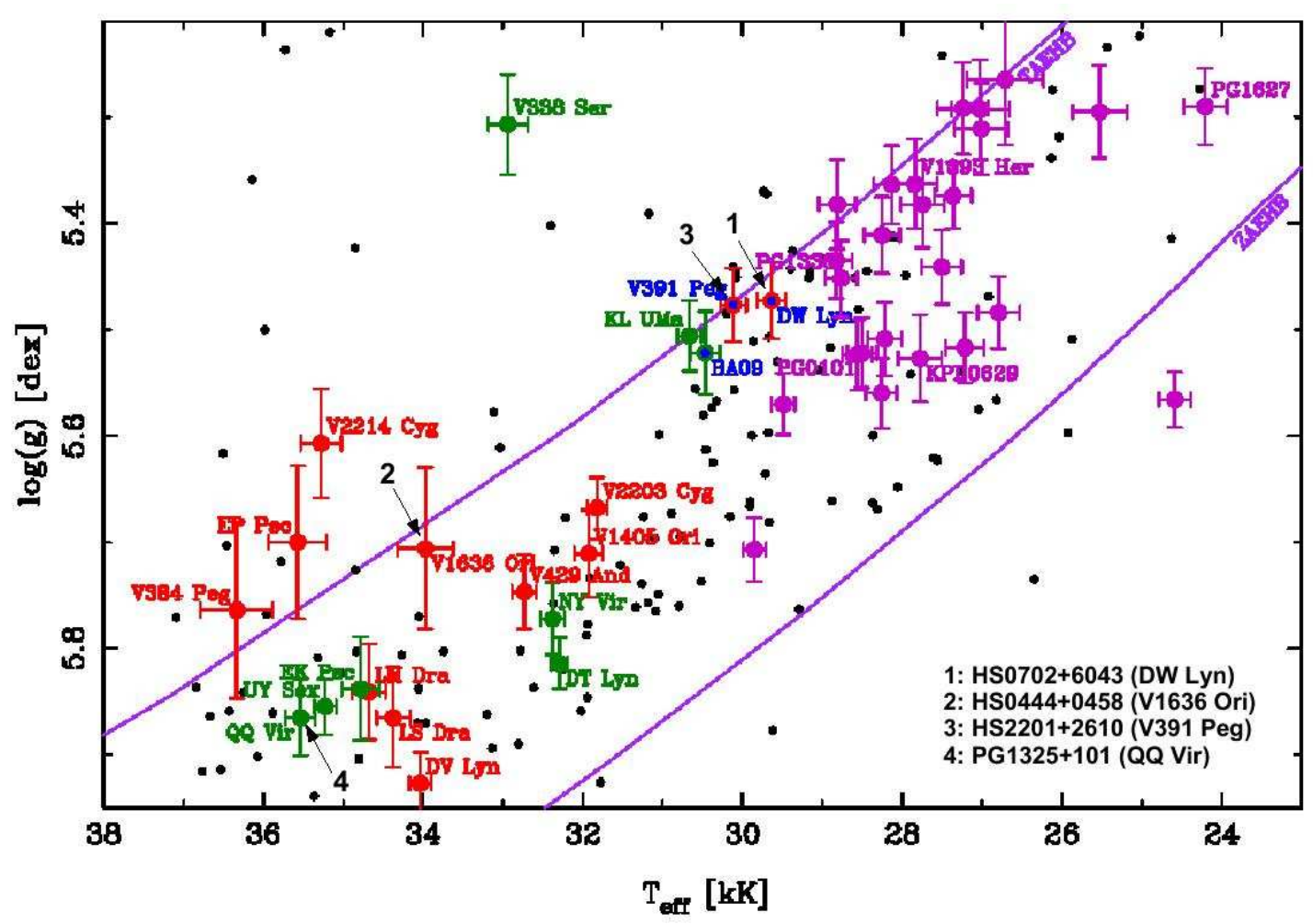

Figure 1.10: $\log q-T_{\text {eff }}$ diagram showing the extreme horizontal branch. SdB pulsators from the Green et al. (2008) sample are shown as the big dots with error bars while the stars represented by the black small dots represent stars showing no observable pulsations. The colors indicate short period $\mathrm{sdBV}_{r}$ pulsators with (green) and without (red) published asteroseismic solution as well as long period $\mathrm{sdBV}_{s}$ pulsators (magenta) and hybrid $\mathrm{sdBV}_{r s}$ pulsators (blue core). Zero- and terminal-age horizontal branch (ZAEHB and TAEHB, respectively) are drawn as solid lines. The objects relevant to this thesis are marked by numbers 1 to 4 and listed in the bottom right. This is a modified version of Østensen (2009).

Let us now point out some differences between the two classes and start with the atmospheric parameters: looking at the location of sdB pulsators in a $\log g-T_{\text {eff }}$ diagram (see Figure 1.10), it becomes obvious that the $\operatorname{sdBV}_{r}$ stars are generally found at higher effective temperatures and higher surface gravities than the $\mathrm{sdBV}_{s}$ stars. The presence of hybrid objects, however, proves that the instability regions are not strictly separated but that there is an overlap region. A similar behavior of overlapping instability regions can also be observed for hybrid $\beta$ Cep $(p)$ - SPB $(g)$ and $\delta$ Sct $(p)-\gamma$ Dor $(g)$ pulsators on the main sequence. From the observer's point of view, it is 
interesting to distinguish the pulsational periods and amplitudes of the excited modes: $\mathrm{sdBV}_{r}$ stars exhibit periods in the range of a few minutes (roughly between 100 and 500 seconds) with amplitudes of a few ten mmag. The $\mathrm{sdBV}_{s}$ stars in contrast pulsate with longer periods between 30 and 90 minutes and also with lower amplitudes (only some mmag). It is obvious that the latter class is much harder to observe by means of ground based observations. The future of $g$-mode asteroseismology therefore lies in space borne observatories, such as the Kepler spacecraft.

Asteroseismology, being the only tool to "look inside a star", has the potential to directly probe the inner structure of a star, since each excited eigenmode has a period depending on the physical structure of the star as a whole. For example, the shallowly penetrating $p$-modes are mostly sensitive to the envelope and should therefore provide information on diffusive processes and metallicities in the envelope, while the $g$-modes penetrate deeper and are therefore more sensitive to core conditions. Finding both types of modes excited in the same object therefore makes the hybrid sdBV $\mathrm{s}_{s}$ stars prime targets for asteroseismic modeling. There are further properties ac-

Table 1.1: Group properties of sdB pulsators. A list of all published rapid sdB pulsators $\left(\mathrm{sdBV}_{r}\right)$ can be found in Table 9 of Østensen et al. (2010). There are five published hybrid sdBV $\mathrm{r}_{r s}$ objects prior to the Kepler spacecraft era: HS 0702+6043, Balloon 090100001, HS 2201+2610, RAT 0455+1305 and J 08069+1527. The Kepler spacecraft discovered 1 new $\operatorname{sdBV}_{r}$ and 14 new $\operatorname{sdBV}_{s}$ stars (Østensen et al., 2011), where many of the latter also show signs of a rapid pulsation, i.e. a hybrid character. Reed et al. (2010) investigate such hybrid signatures in sdB targets within the Kepler field of view. For the group classification I use the proposed nomenclature of Kilkenny et al. (2010).

\begin{tabular}{c|cc}
\hline property & sdBV $_{r}$ & $\mathrm{sdBV}_{s}$ \\
\hline period range & few minutes & $\approx 30-90$ minutes \\
amplitudes & up to $\approx 50 \mathrm{mmag}$ & few mmag \\
effective temperatures & $\approx 28000-38000 \mathrm{~K}$ & $\approx 22000-30000 \mathrm{~K}$ \\
surface gravities $\log g$ & $\approx 5.4-6.2$ & $\approx 5.2-5.6$ \\
mode type & acoustic pressure $p$-modes & gravity $g$-modes \\
mode properties & low degree, low radial order & low degree, high radial order \\
probing region & shallow, sensitive to envelope & deep, sensitive to core \\
driving & $\kappa$-mechanism & $\kappa$-mechanism \\
$\#$ (ground based) & 49 & ca. 30 \\
$\#($ Kepler $)$ & 1 & 14 \\
\hline
\end{tabular}


cessible via asteroseismology that can provide physical information on these stars: a frequency splitting for example could be referred to rotation or magnetic fields and mode trapping effects, which perturb the period distribution, might be indicative for the transition boundary between the helium core and the hydrogen envelope. The full potential of sdB asteroseismology and the principle of the modeling is reviewed in Fontaine et al. (2006) and Charpinet et al. (2009), respectively. Full case studies can be found e.g. in Charpinet et al. (2006), Randall et al. (2009) or Van Grootel et al. (2010). Based on four primary quantities in their models $\left(\log g, T_{\text {eff }}\right.$, total mass and mass of the envelope), the above authors used period matching to derive secondary properties like radius, luminosity, absolute visual magnitude, distance, rotation period, equatorial velocity and inclination. Such full asteroseismic solutions exist for a handful of sdB pulsators. These asteroseismic mass determinations (cf. Figure 1.3) and the mass distributions are of course also of particular interest in the context of $\mathrm{sdB}$ formation to test the different formation channels.

After highlighting the potential of sdB asteroseismology, I finally want to conclude this section with the summary of the differences between the two groups of sdB pulsators given in Table 1.1.

\subsection{Exoplanets}

In the present section I provide a short general overview of the exoplanets found to date and point out the most intriguing statistical and dynamical properties. To put this into the context of the present thesis, I will focus on evolved planetary systems and emphasize the differences between these and "non-evolved" systems.

In contrast to the precise definition of a "planet" in the Solar System, there is no such generally accepted definition of what an "exoplanet" is. However, in a position statement on the definition of a planet, the working group on extrasolar planets of the international astronomical union states:

"objects with true masses below the limiting mass for thermonuclear fusion of deuterium (currently calculated to be 13 Jupiter masses for objects of solar metallicity) that orbit stars or stellar remnants are "planets" (no matter 
how they formed)." and further:

"substellar objects with true masses above the limiting mass for thermonuclear fusion of deuterium are "brown dwarfs", no matter how they formed nor where they are located". Another suggestion is to rather distinguish exoplanets from brown dwarfs based on their formation mechanism instead of applying the deuterium fusion threshold.

Also, if the former criterion is applied, the 13 Jupiter masses should not be regarded as a hard limit since the onset of deuterium burning not only depends on the mass but also on the composition and chemistry. For example, two popular exoplanet databases, the "extrasolar planets encyclopaedia"6 and the "exoplanet data explorer", include objects with masses up to 25 or 24 Jupiter masses in their database, respectively. A fact to keep in mind is that for many exoplanet candidates, due to unknown inclinations, only lower mass limits are known instead of true masses.

Beside all this, the definition of a substellar object is generally accepted to be an object not able to sustain stable hydrogen burning, which is the case for masses approximately below 0.075 solar masses (Baraffe et al., 2002).

\section{Current sample}

As of 15.04.2011 the "extrasolar planets encyclopaedia" listed 543 exoplanets confirmed in the literature. Among these are 498 exoplanets detected with the radial velocity method (RV). This number already includes 127 exoplanets found with the transit method. These two methods turned out to be most successful in terms of number of detections. In addition there are 12 exoplanets found using a microlensing technique, 21 by direct imaging and 12 by a timing method. Figure 1.11 shows the current exoplanet sample coded according to the detection method.

When comparing these numbers, one always has to keep in mind the different sensitivities and observational biases/selection effects of the various methods. Concerning these aspects, I will go into detail in a later chapter when I describe the timing method used in this study.

${ }^{6}$ http://exoplanet.eu/

'http://exoplanets.org/ 


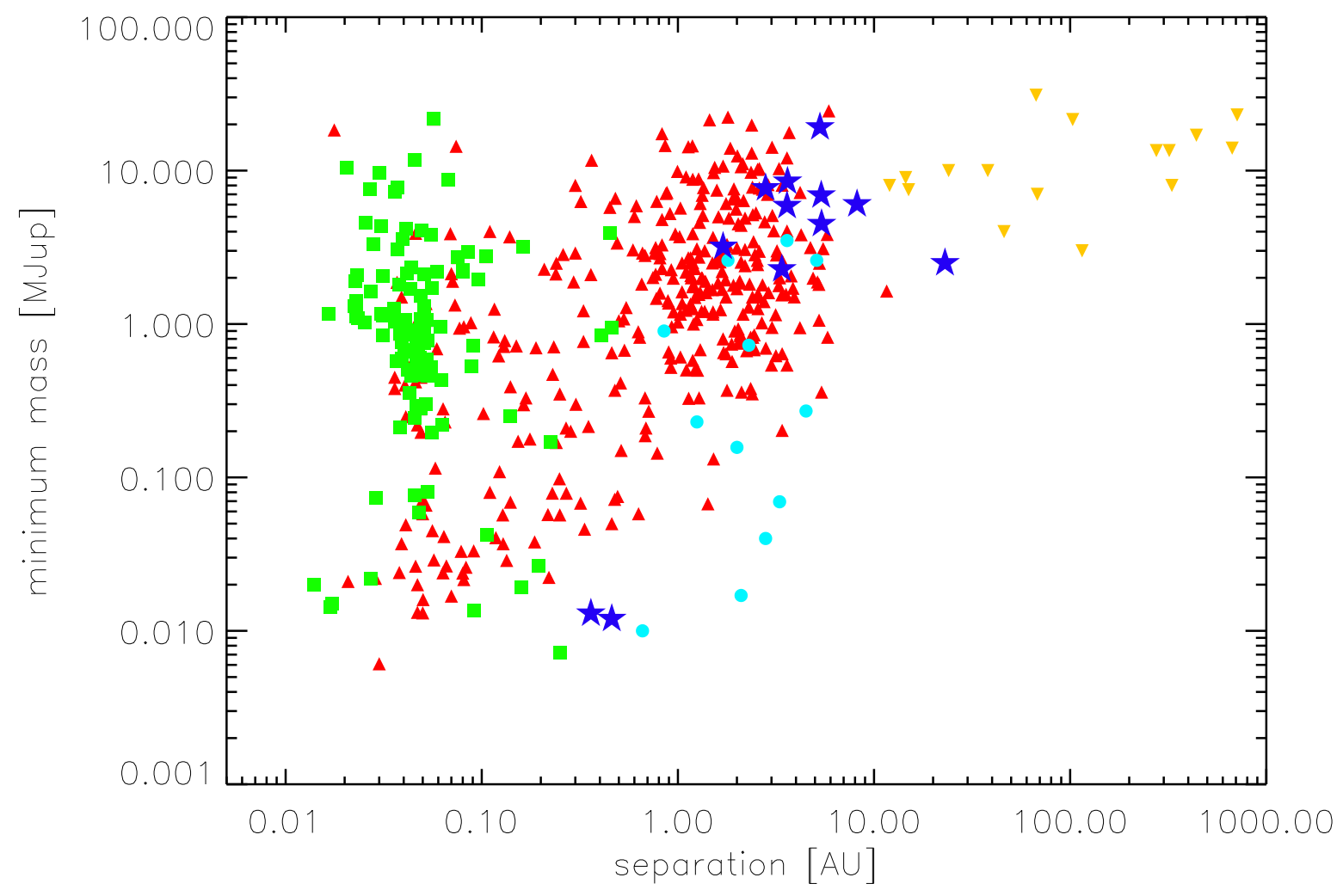

Figure 1.11: Location of exoplanets on the mass versus separation plane according to the detection method. The methods included are the transit method (green squares), the RV method (red upward triangles), microlensing (light blue circles), direct imaging (orange downward triangles) and the timing method (dark blue stars). Data are taken from http://exoplanet.eu/. The planet PSR 1257+12 b with $0.00007 M_{\text {Jup }}$ at $0.19 \mathrm{AU}$ is not drawn here since it is off the scale.

An imbalance becomes obvious when looking at the stellar types which host exoplanets. As can be inferred from Figure 1.12 the vast majority of host star types is located on the main sequence or just evolved off the main sequence to become a sub-giant or giant. The only published and confirmed evolved exoplanet host stars at the time of writing are HS 2201+2610 (apparently single $\mathrm{sdB}$ of $\mathrm{sdBV}_{r} s$ type) and HIP 13044 (red HB star) as single host stars and a longer list of binary host stars: HW Vir (binary sdB $+\mathrm{dM}$ of Algol type), DP Leo (binary WD+dM of AM Her type), NN Ser (binary $\mathrm{WD}+\mathrm{dM}$ of post-CE or pre-CV type), HU Aqr (binary WD+dM of AM Her type), UZ For (binary WD+dM of AM Her type). This list of evolved host 

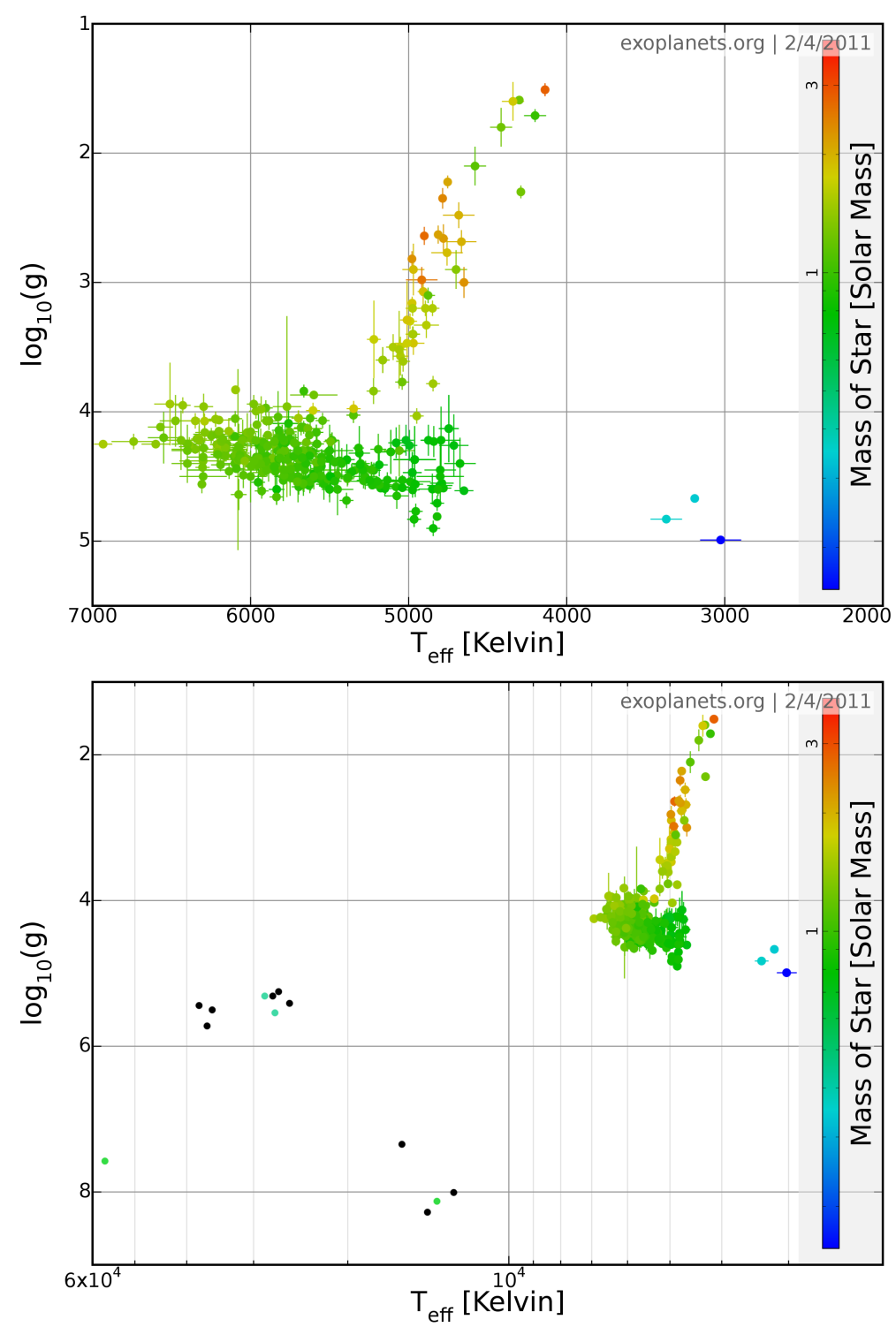

Figure 1.12: Exoplanet host stars. Top panel: position of host stars of 427 exoplanets, which were found with the radial velocity or transit method. The color scales with the mass of the host star. Stellar types are main sequence, subgiant or giant, i.e. non-evolved or just evolved off the MS. Bottom panel: Same as top, but with the inclusion of evolved host stars of exoplanets (the four colored dots on the left hand side: HS 2201+2610, HW Vir, NN Ser and DP Leo). Note the change in the axis ranges. Evolved host stars of brown dwarf companions (some are only candidates) are also included as black dots, in order to complete the current picture of substellar companions to evolved stars. The pulsar host stars PSR B1257+12 and PSR B1620-26 of planetary companions are not included the plot because of their surface gravities (in the range $\log g \approx 14$ ) being out of the plotting range. The tool to produce these plots is described in Wright et al. (2011). 
stars is completed by the two pulsars PSR B1257+12 and PSR B1620-26. A further but yet controversial case is QS Vir (binary WD+dM of Algol type). There are some more candidate studies under way, mainly for HWVir and W UMa type systems.

In addition, a couple of brown dwarfs and brown dwarf candidates are known to orbit evolved stars (cf. bottom panel of Fig. 1.12).

\section{Statistics on physical and dynamical properties}

In brevity, I now want to mention some conclusions drawn from studying the physical and dynamical properties of the exoplanetary systems known to date. This will be an incomplete overview and does not include details, special cases, exceptions etc. It is solely meant to describe some general trends.

The "extrasolar planets encyclopaedia" at http://exoplanet.eu/ provides a plotting tool which allows to plot histograms and to correlate several properties of the current exoplanet sample. By correlating basic properties like mass, orbital period, eccentricity, semi-major axis etc. one can find some basic trends:

Looking at the mass distribution of exoplanets by number, one finds a steep rise from the highest masses towards lower masses with the largest number of planets found in the interval roughly between 0.5 and $1.5 M_{\text {Jup }}$. This is somehow counter intuitive since larger masses should be easier to detect but it might suggest that lower mass exoplanets are more abundant than higher mass exoplanets.

The period- (and semi-major axis) distributions show a distinct bi-modality. There appear strong peaks around three days (corresponding to $0.04 \mathrm{AU}$ ) and roughly 250 days (corresponding to $0.8 \mathrm{AU}$ ), whereas periods/semimajor axes in between seem to be rarer. The same holds for very short $(<1$ day) and very long ( $>1$ year) periods. The short period peak is suspected to be due to migration effects, but this hypothesis still has be investigated in more detail and it still has to be shown if this is due to an observational bias caused by the applied detection method.

Correlating the exoplanetary masses with their periods, one finds a lack of very massive planets ( $>$ two Jupiter masses) on short period orbits $(<100$ days or $0.4 \mathrm{AU})$. This could perhaps be explained by evaporation effects or 
in-fall of massive planets into the host stars. However, lower mass planets ( $<$ two Jupiter masses) are found at all distances ranging from days to years. A comparison of the eccentricity of exoplanets with their period yields the result that basically all short period orbits $(<$ six days) are circularized. For larger orbital periods all eccentricity values between zero and one are present, with a median around 0.24 for periods longer than ten days (0.09 AU). In this context, our Solar System planets show unusually low eccentricities.

The probability of finding a giant planet increases with the host star's metallicity. No such trend is found for low-mass (solid) planets, but in the latter case the statistics have to be improved. In addition, no such metallicity trend with the semi-major axis is found.

Also the probability for finding an exoplanet seems to be higher for highermass host stars, and low-mass exoplanets seem to be more common around M-dwarfs. It still has to be shown if these are observational biases due to sample limitations.

Multiple exoplanet systems tend to be densely packed and to build a compact configuration with a regular spacing on a logarithmic semi-major axis scale. However, such a hypothetical "extrasolar Titus-Bode law" is still under debate in the literature.

\section{Evolutionary aspects}

As already stated in the section concerning sdB formation channels, a close proximity of exoplanets to their host stars may lead to a significant mass loss of the stars at later stages of the evolution. The interesting aspect comes in, as the host star evolves off the main sequence and the question how an exoplanetary companion may influence the further evolution of the host star. Following Soker (1998), exoplanets (or substellar companions in general) in orbits up to $5 \mathrm{AU}$ can interact with the expanding red giant envelope and enhance the host's mass loss by deposition of angular momentum and energy to the stellar envelope. As mentioned before, this mechanism is very promising to explain apparently single sdB stars. In this context, sdB stars are very attractive for yet another reason: once an exoplanetary companion is found to an sdB star, one can be sure that this planet will not have to struggle with violent AGB phases, thermal pulses and PN phases of its host 
star since the subdwarfs do not take this evolutionary path as stated earlier, but directly evolve to the white dwarf cooling sequence. It will therefore have a higher survivability. In addition, if the planet is found around a single sdB star instead of being part of a binary star system it will also not be influenced by possible RLOF or CE phases of the binary components. It is therefore plausible to assume that a planet orbiting a (single or binary) sdB star, if not formed as a "second-generation planet" in a merger scenario, has survived the first Red Giant phase of the host star and that a planet orbiting a single sdB star will also survive the rest of its host stars' evolution.

Livio \& Soker (2002) conclude that $\approx 4-10 \%$ of solar-type stars will be significantly affected (i.e. experience enhanced mass loss) by substellar companions during their further evolution. Analyzing the occurrence of exoplanets as a function of stellar mass, Marcy et al. (2008) find 4\% of solar-type stars to harbor planets within 2.5 AU and Bowler et al. (2010) mention that 26\% of evolved A-type stars host Jupiter-mass planets within 3 AU. Having this in mind, it is not only interesting to consider the influence of the host star on an eventual planetary companion but, vice versa, also to consider the influence of the exoplanet on the evolution of the host star. As we have seen in the case of single sdB stars the presence or absence of a substellar companion may define two totally different evolutionary scenarios for the same host star.

\section{Planetary systems around evolved stars}

As I sketched earlier, very little is known about planetary systems around evolved stars, both on the theoretical and in particular on the observational side. However, both sides recently made a lot of progress and, although still being in its infancy, there is a promising development on the way. Here I want to refer to recent studies:

From the theoretical point of view, Villaver (2011) investigates the fate of planets for canonical host star evolution and Perets (2010, 2011) puts a special focus on second-generation planets and planets in evolved binary systems.

Studies concerning planets around giant stars (Quirrenbach et al., 2011; 
Omiva et al., 2011; Jones et al., 2011) and debris disks and brown dwarfs orbiting white dwarfs (Farihi, 2011; Burleigh et al., 2011) have also been published recently.

Observational evidence for exoplanets around evolved host stars (precisely such host stars which have already experienced the helium flash) is given for HS 2201+2610, aka V391 Peg (Silvotti et al., 2007), for HW Vir (Lee et al., 2009), for DP Leo (Qian et al., 2010; Beuermann et al., 2011), for NN Ser (Qian et al., 2009a: Beuermann et al.. 2010). for PSR B1257+12 (Wolszczan \& Frail, 1992; Wolszczan, 1994) and for PSR BB1620-26 (Thorsett et al., 1993; Backer et al., 1993).

Recently, Setiawan et al. (2011) announced the discovery of an exoplanet orbiting the metal-poor star HIP 13044 on the Red Horizontal Branch, which is probably of extragalactic origin (see also Bear et al., 2011).

In their Table 1, Silvotti et al. (2011) list the currently known post-RGB exoplanetary systems (with the addition of brown dwarf companions to complete the picture of substellar companions). A controversial discussion whether or not the sdB HD 149382 has a substellar companion can be found in Geier et al. (2009a) and Jacobs et al. (2011). Also the pulsating DAV white dwarf GD 66 is a promising yet unconfirmed candidate to harbor a substellar companion (Mullally, 2007; Mullally et al., 2008, 2009).

Observational progress in adapting the RV method to sdB stars is presented in O'Toole et al. (2011) and Classen et al. (2011) and further brown dwarf candidates to sdB stars are presented in Geier et al. (2011a) and Schaffenroth et al. (2011), respectively.

Finally, the current evolved exoplanets are put into a broader context by Silvotti (2009) and Bear \& Soker (2011b). Bear \& Soker (2011a) compare the currently known post-RGB planets to the main-sequence population.

I want to conclude this section with the preceding collection which is intended to present the current status in the studies of evolved planetary systems. 


\section{Chapter 2}

\section{Motivation}

Being now provided with the basic background knowledge for the course of this thesis, the following chapter intends to build a bridge. Based on the information given in the previous chapter, I aim to introduce the ideas and motivate the concepts I want to use in the following to investigate the subdwarf B stars in this work.

As we have learned in the preceding chapter, hot subdwarf B stars can be identified as evolved objects located on the extreme horizontal branch which burn helium in their cores. During the evolution on the red giant branch the progenitor must suffer an extreme mass loss, such that at the time of the helium flash only a tiny hydrogen envelope is left. This envelope is inert and subsequently prevents the star to evolve towards the asymptotic giant branch but instead places it at the very hot end of the horizontal branch. Bypassing also the planetary nebula phase, the sdBs are believed to directly evolve to the white dwarf cooling sequence.

There is also the possibility that sdB stars exhibit pulsations. Two different types of modes can appear: short period pressure $(p-)$ modes or long period gravity $(g-)$ modes. For this thesis the former group, the rapidly pulsating $s d B V_{r}$ stars, will be of importance. The analysis of these stellar oscillations will allow us to investigate two major aspects of this thesis:

- to search for substellar companions (exoplanets and/or brown dwarfs) around our targets and

- to constrain the evolutionary status of our targets.

The former aspect is connected to exoplanet research. Due to limitations 
given by the common detection methods and the desire to find an Earthanalogon in a solar-system-like exo-system, most of the exoplanet surveys are focused on solar-like stars on the main sequence. However, little is known about the late stages of exoplanetary system evolution, in particular the fate of exoplanets during and after the red giant expansion of the host star. The number of exoplanets around giant and subgiant stars is increasing, but still low compared to the Main Sequence host stars. The same is true for white dwarfs and for hot subdwarfs, the target objects of this work. Finding exoplanets around these stars could be seen as an immediate proof that exoplanets would be able to survive the host star expansion or even a common envelope phase ${ }^{1}$. As stated earlier, the formation of sdB stars is not very well understood: the binary sdB population can quite well be explained by the current binary formation channels including common envelope evolution and stable and unstable RLOF, however there are issues to explain apparently single sdB stars. The most promising scenario for single sdB formation is enhanced mass loss of the sdB progenitor during the red giant phase induced by substellar companions. Therefore, finding substellar objects around single sdB stars would directly support this hypothesis. Our immediate aim is therefore to apply a timing method to search for substellar companions around pulsating sdB stars which would reveal themselves by the orbital reflex motion they induce on their host stars.

The sdB star HS $2201+2610$ is the first and only hot subdwarf so far where a pulsation timing analysis led to the discovery of an exoplanetary companion (minimum mass of 3.2 Jupiter masses in an orbit of 1170 days). If this exoplanet formed as a first generation planet at the same time as the host star, it would be direct proof that the substellar object survived the red giant expansion of its host star. Triggered and motivated by this exciting discovery, the EXOTIME program was set up. EXOTIME is an integral part of the present work and aims to find more evolved exoplanetary systems around hot subdwarf B stars. In this context a so-called $\mathrm{O}-\mathrm{C}$ diagram will be introduced. Substellar objects around sdB stars are important for two different reasons: they are suspected to be able to influence the evolution of their host-star and they are an ideal test case to examine the properties of exoplanets which have survived the red giant expansion of their host stars.

${ }^{1}$ This only holds if a second generation origin of the planet can be excluded. 
Another aspect is connected to the stellar evolution. Examining the secular changes in the pulsations (i.e. the temporal behavior of the pulsation periods, the so-called $\dot{P}$ ) is an interesting approach which can provide useful input and constraints for asteroseismological models in order to characterize the stellar internal structure. It also can be used for mode identification as well as to pin down the evolutionary status by measuring $\dot{P}$ directly, and therefore determining the evolutionary timescale. Interestingly, the same data used for the exoplanet approach as an extrinsic perturbation can also be used in this asteroseismic context by analyzing the intrinsic temporal behavior of the pulsations. Concerning sdB stars, there are only two objects with published $\dot{P}$ measurements so far.

Based on this present motivation, the following chapters will introduce the process of obtaining and processing the data needed to conduct our analysis. I will further introduce the basic methodology and concepts being part of the pulsation timing and finally present the results of our search for exoplanets and the attempts to measure $\dot{P}$. A subsequent discussion of implications and applications will put the results into a broader context. 


\section{Chapter 3}

\section{EXOTIME}

In a nutshell, the goals of the EXOTIME program (EXOplanet search with the TIming MEthod) are stated in the following citation adapted from the EXOTIME webpage ${ }^{1}$ :

Exoplanet research is strongly focused on main sequence stars similar to our Sun, with the main goal of finding Earthlike planets in the habitable zone, and on planetary system's formation. What can happen to the inner planets at the end of the stellar evolution is largely unknown. Following the example of V391 Peg b (Silvotti et al., 2007), the main goal of this project is to detect planets orbiting evolved pulsating stars such as subdwarf B (sdB) stars or white dwarfs (WDs) through the timing method, using the stellar pulsation as a clock. Transits and radial velocities are not efficient for stars with small radii and high gravities. The secondary goal of this project is asteroseismology: characterization of the targets through seismic methods and in particular the measurement of $\mathrm{dP} / \mathrm{dt}$ (the secular variation of the pulsation periods), which allows a precise determination of the evolutionary status of a star and can help the identification of the pulsation modes. This project can contribute to explain the formation of single sdB stars: sub-stellar objects might contribute to enhance mass loss from the sdB progenitor near the tip of the red giant branch. The EXOTIME

${ }^{1}$ http://www.na.astro.it/〜silvotti/exotime/ 
collaboration is based on a network of ground-based 1-4m class telescopes.

Given this basic idea, I will expand to a more detailed description in the following sections.

\subsection{Description and status of the project}

With the idea to directly measure secular variations in the pulsations of variable subdwarf B stars, a long-term monitoring of the object HS 2201+2610 (V 391 Peg) was started in 2002. The huge photometric data set gathered since then also made it possible to search for orbital signatures of possible substellar companions. Indeed, Silvotti et al. (2007) announced the first exoplanet discovered to orbit an evolved star (besides the pulsar planets). Encouraged by this detection, the authors initiated the EXOTIME program in 2008 by increasing the target list. It currently comprises the objects HS 2201+2610, HS 0444+0458, HS 0702+6043, PG 1325+101 and EC 09582-1137. The responsibility for all data and analysis connected with the two targets HS $0444+0458$ and HS $0702+6043$ is matter of the present thesis.

Current status reports of the project can be found in a general overview by Schuh et al. (2010) and for individual targets in Lutz et al. (2011) for HS 0444+0458 and HS 0702+6043 and Benatti et al. (2010) for PG 1325+101.

The analysis intended to be applied within the EXOTIME program requires a regular long-term photometric monitoring of all our targets (details of the requirements will follow in the data and methods chapters). It is therefore designed to be a multi-site project covering several observatories equipped with small- to medium class telescopes in the range of one to four meter mirror sizes.

\subsection{Targets}

To determine whether an object is a suitable target for long-term monitoring, one has to consider two groups of characteristics: practical and physical 
properties. For the practical properties, since we conduct ground-based observations with telescopes on the northern hemisphere, we have to check the yearly visibilities of the targets. This is shown as an example for the MONET/N site in Fig.3.1. As can be seen, the RA distribution of the EXOTIME targets (labeled with 1 to 5 in Fig.3.1) covers the whole year. The targets analyzed in this thesis, HS $0702+6043$ and HS $0444+0458$, are labeled with 1 and 2 in Fig. 3.1 and are only observable from approximately September until April/May. This is also the case for the other sites and has of course to be kept in mind when scheduling the multi-site observations. Additionally, the B-magnitudes (the maximum energy emitted by sdB stars lies in the blue/UV wavelength regime) must not be too faint, such that the potential targets can still be observed with small class telescopes.

Concerning the physical properties, a basic factor for a pulsating sdB star to be examined for substellar companions with the timing method is the characteristic of its periodogram. A suitable target shows not too many frequencies, moderately large and stable amplitudes and a suitable magnitude. Most importantly, the pulsations must not show any severe deviations from phase coherence. The argument with the moderate number of frequencies is based on the desire that one wants to have more than one frequency to allow independent measurements of the evolutionary timescale and possible orbital configurations of companions. Contrariwise, we want to avoid having too many frequencies since the possibility of beating effects, which may cause contamination in the $\mathrm{O}-\mathrm{C}$ diagrams, becomes larger as the number of frequencies grows. Ideally, we would like to have three to four dominant and, most importantly, independent frequencies which are easily resolvable even in short runs (2-3 nights). The amplitudes should be large enough to be detectable even with small class telescopes $(>1 \mathrm{mmag})$ but still small enough to avoid significant non-linear effects. However, given the generally small pulsational amplitudes of $p$-mode sdB pulsators ( $<50 \mathrm{mmag}$ ), nonlinearity should not be of major importance. Amplitude stability analyses of some sdBV stars have been conducted by Kilkenny (2010) with the result that amplitude variability seems to be a common effect and has to be considered carefully in studies attempting to measure secular period changes. 
Figure 3.1: Visibility of the EXOTIME targets throughout the year for MONET/N. Plot produced by http://catserver.ing.iac.es/staralt/index.php. 
Since we are interested in the evolution of single sdB stars, known binary sdBs and sdBs with significant IR-excess have to be dismissed. This point will be addressed in detail in the subsequent section.

Considering all the aspects mentioned above, the following five objects are the most promising ones and have been selected as EXOTIME targets by the initiators R.Silvotti and S.Schuh. I will list some relevant papers on each of the EXOTIME targets:

\section{- HS 0444+0458:}

HS 0444+0458 was discovered as pulsating sdB star by Østensen et al. (2001a). Reed et al. (2007) conduct a frequency analysis.

\section{- HS 0702+6043:}

HS $0702+6043$ was first identified as a $p$-mode variable in a search program by Dreizler et al. (2002). Schuh et al. (2006) discovered additional $g$-mode pulsations, making this star the prototype of hybrid sdB pulsators and triggering extensive observational follow-up (Lutz et al., 2008; Francoeur et al., 2010).

\section{- HS 2201+2610:}

Discovered by Østensen et al. (2001b), results from a time-series campaign on this object are given in Silvotti et al. (2002a). Silvotti et al. (2007) found HS 2201+2610 to host a planetary companion, and possible evolutionary scenarios for this system are discussed in Silvotti (2008). Lutz et al. (2009) added this star to the hybrid group of sdB pulsators and Silvotti et al. (2010) report on multicolor photometry and mode identification.

\section{- PG 1325+101:}

This sdBV star is first addressed in Silvotti et al. (2002b). Results on frequency analvsis and asteroseismological modeling are given by Silvotti et al. (2006) and Charpinet et al. (2006), respectively. Baran et al. (2010) conduct a mode identification and Benatti et al. (2010) comment on efforts of long-time monitoring within the EXOTIME program.

\section{- EC 09582-1137:}

EC 09582-1137 was discovered by Kilkennv et al. (2006) and Randall et al. (2009) conduct an asteroseismological analysis of this target. 
Table 3.1: EXOTIME targets. Coordinates are taken from http://simbad.u-strasbg.fr/simbad/. References for masses and atmospheric parameter: Dreizler et al. (2002). Ostensen et al. (2001a). Charpinet et al. (2006). Silvotti et al. (2007) and Randall et al. (2009) for the targets from left to right, respectively. The masses result from asteroseismic modeling. Otherwise, if no error is given, we assume a canonical mass of $0.5 M_{\odot}$.

\begin{tabular}{|c|c|c|c|c|c|}
\hline & HS $0702+6043$ & HS 0444+0458 & PG $1325+101$ & HS $2201+2610$ & EC 09582-1137 \\
\hline Alias & $\mathrm{V}^{*}$ DW Lyn & $\mathrm{V}^{*}$ V1636 Ori & $\mathrm{V}^{*} \mathrm{QQ}$ Vir & $\mathrm{V}^{*}$ V391 Peg & PG 0958-116 \\
\hline RA (2000) & $07^{h} 07^{m} 09^{s} .8$ & $04^{h} 47^{m} 18^{s} .6$ & $13^{h} 27^{m} 48^{s} \cdot 6$ & $22^{h} 04^{m} 12^{s} .2$ & $10^{h} 00^{m} 41^{s} .8$ \\
\hline Dec (2000) & $+60^{\circ} 38^{\prime} 50^{\prime \prime}$ & $+05^{\circ} 03^{\prime} 35^{\prime \prime}$ & $+09^{\circ} 54^{\prime} 52^{\prime \prime}$ & $+26^{\circ} 25^{\prime} 08^{\prime \prime}$ & $-11^{\circ} 51^{\prime} 35^{\prime \prime}$ \\
\hline B-mag & 14.7 & 15.2 & 13.8 & 14.3 & 15.0 \\
\hline V-mag & 15.1 & 15.4 & 14.02 & $14.57 \pm 0.02$ & 15.26 \\
\hline J-H color & -0.123 & 0.043 & -0.142 & 0.004 & -0.243 \\
\hline$T_{\text {eff }}[\mathrm{K}]$ & $28400 \pm 600$ & $33800 \pm 1000$ & $35050 \pm 220$ & $29300 \pm 500$ & $34806 \pm 233$ \\
\hline $\log g\left[g\right.$ in $\left.\mathrm{cm} \cdot \mathrm{s}^{-2}\right]$ & $5.35 \pm 0.10$ & $5.60 \pm 0.15$ & $5.81 \pm 0.04$ & $5.4 \pm 0.1$ & $5.80 \pm 0.04$ \\
\hline $\log (\mathrm{N}(\mathrm{He}) / \mathrm{N}(\mathrm{H}))$ & $-2.7 \pm 0.1$ & $-1.85 \pm 0.20$ & $-1.70 \pm 0.02$ & $-3.0 \pm 0.3$ & $-1.68 \pm 0.06$ \\
\hline pulsational class & $\mathrm{sdBV}_{\mathrm{rs}}$ & $\mathrm{sdBV}_{\mathrm{r}}$ & $\mathrm{sdBV}_{\mathrm{r}}$ & $\mathrm{sdBV}_{\mathrm{rs}}$ & $\mathrm{sdBV}_{\mathrm{r}}$ \\
\hline main period $[\mathrm{s}]$ & 363 & 137 & 138 & 350 & 136,151 resp. \\
\hline main amplitude [mmag] & 25 & 5 & 26 & 10 & 8, 7 resp. \\
\hline $\operatorname{mass}\left[M_{\odot}\right]$ & 0.5 & 0.5 & $0.50 \pm 0.01$ & $0.50 \pm 0.05$ & $0.485 \pm 0.011$ \\
\hline radius $\left[R_{\odot}\right]$ & 0.247 & 0.186 & 0.146 & 0.23 & 0.147 \\
\hline distance $[\mathrm{pc}]$ & 2042 & 1894 & 784 & 1483 & 1494 \\
\hline luminosity $\left[L_{\odot}\right]$ & 35.7 & 40.3 & 28.7 & 36.1 & 28.6 \\
\hline abs. bolometric magnitude & 0.85 & 0.72 & 1.08 & 0.84 & 1.09 \\
\hline bolometric correction & -2.70 & -3.30 & -3.47 & -2.88 & -3.30 \\
\hline
\end{tabular}


We summarize the known parameter of all EXOTIME targets in Table 3.1 . For the atmospheric parameters, I also want to point to Fig. 1.10. In addition, field of view snapshots as well as example light curves and example periodograms of our targets can be found in the appendix.

\subsubsection{IR colors}

If a hot subdwarf B star has a cool stellar companion, the latter will most easily be revealed by its contribution to the IR part of the composite spectrum. An extensive study dealing with 2MASS colors and hot subdwarf stars is presented in Stark \& Wade (2003). However, in order to exclude cool stellar companions to our targets, i.e. to make sure that they are single sdB stars, we want to compare the measured 2MASS JHK colors of our targets to their expected energy distributions. For a sdB star a blackbody radiation in the optical part of the spectrum is a valid approximation. To get the target's blackbody flux at Earth, one has to know both the radius and the distance of the sdB star. With the given surface gravities and an assumed canonical sdB mass of $0.5 M_{\odot}$ we can calculate our target's radii according to $R=\sqrt{G M / g}$, with $G$ being the gravitational constant.

To estimate the distances from our targets, we first calculate the luminosities as $L=4 \pi \sigma \cdot R^{2} \cdot T_{\text {eff }}{ }^{4}$, putting in the radius $R$, the given effective temperature $T_{\text {eff }}$ and using the Stefan-Boltzmann-constant $\sigma=5.67 \cdot 10^{-8} \mathrm{~W} / \mathrm{m}^{2} / \mathrm{K}^{4}$. From that, one can calculate the absolute bolometric magnitude as $M_{b o l}=$ $M_{b o l, \odot}-2.5 \log \left(L / L_{\odot}\right)$ and in a further step the absolute visual magnitude $M_{V}=M_{b o l}-B C$, which is necessary to calculate the distance modulus. We neglect extinction and reddening and for deriving the absolute visual magnitude from the absolute bolometric magnitude, we need to apply a bolometric correction $B C$. There exist several empirical charts for bolometric corrections as a function of effective temperature and we use the one given in Table 1 in Torres (2010). Using this bolometric correction, we have to use the value 4.73 for $M_{b o l, \odot}$, which is further specified in Torres (2010).

Finally, the distance $d$ can be calculated according to $d[p c]=10^{\left[\left(V-M_{V}\right)+5\right] / 5}$. $V$ and $M_{V}$ are the apparent and absolute visual magnitudes, respectively. All quantities used for the distance determination are given in Table 3.2. 


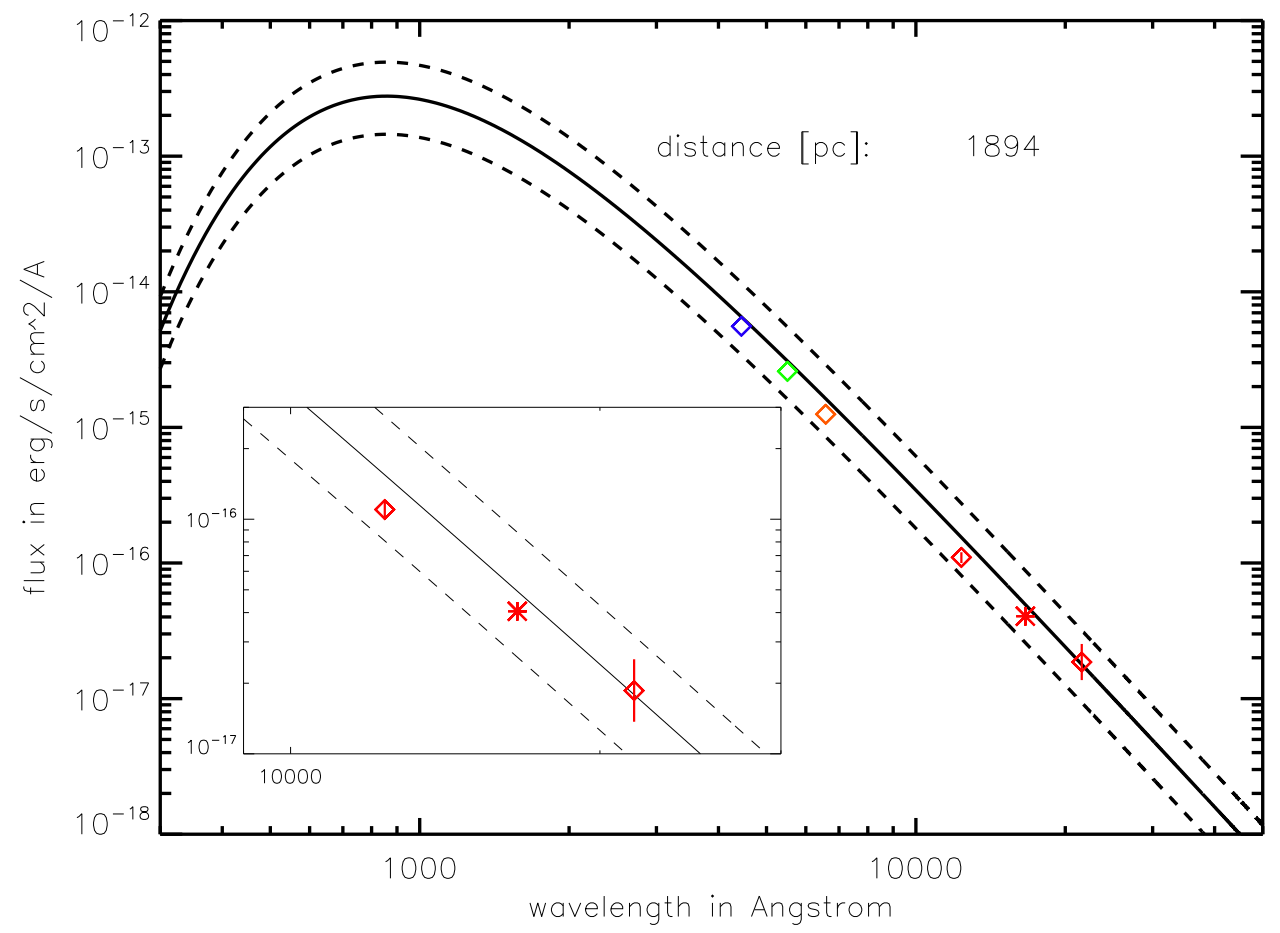

Figure 3.2: Blackbody radiation for HS 0444+0458 compared to BVRJHK colors.

Now being provided with radii and distances, we can calculate the blackbody flux at Earth and compare it to the measured BVRJHK colors. This comparison has been done for all our targets and is shown in Figures 3.2 3.4. As can be seen, there are no significant IR excesses. In each plot we give the distance as calculated according to the procedure described above. The inset windows show a zoom into the IR region with the JHK colors. Error bars are given as vertical lines. The 2MASS archive marks the Hmagnitude of HS 0444+0458 and the K-magnitude of EC 09582-1137 as bad quality data and does not provide errors. In the plots, these bad quality points are shown as a star symbol without error bars. The dashed lines represent the blackbody solutions for the minimum and maximum distances listed at the bottom of Table 3.2 and take into account the uncertainties of all the quantities used in the distance determination. 

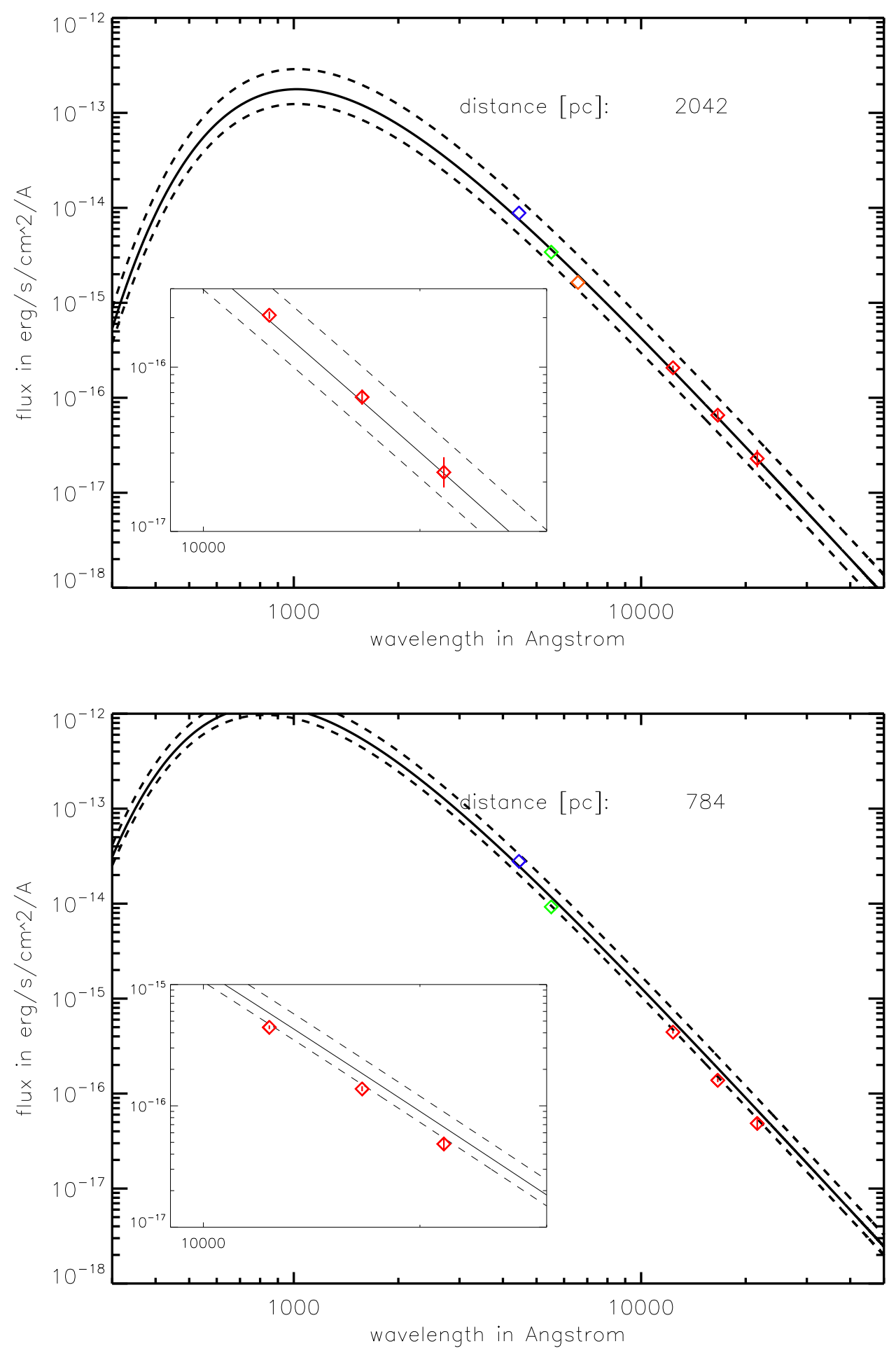

Figure 3.3: Blackbody radiation for HS 0702+6043 (top) and PG 1325+101 (bottom) compared to BVRJHK colors. 

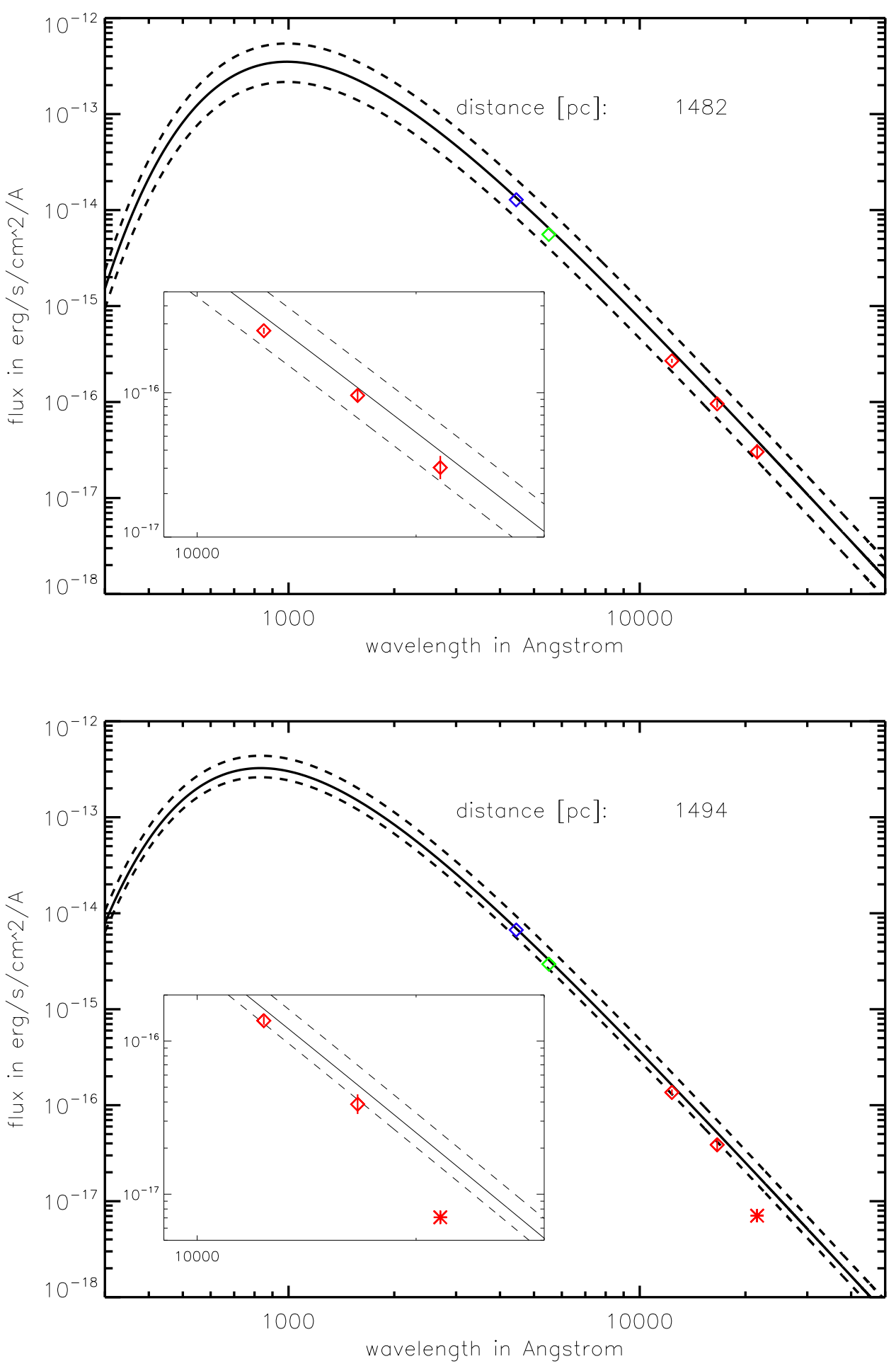

Figure 3.4: Blackbody radiation for HS 2201+2610 (top) and EC 09582-1137 (bottom) compared to BVJHK colors. 
Table 3.2: Quantities used for the distance determination.

\begin{tabular}{|c|c|c|c|c|c|}
\hline Alias & $\begin{array}{r}\text { HS 0702+6043 } \\
\text { V* DW Lyn }^{*} \text { DW }\end{array}$ & $\begin{array}{l}\text { HS } 0444+0458 \\
V^{*} V 1636 \text { Ori }\end{array}$ & $\begin{array}{r}\text { PG } 1325+101 \\
V^{*} \text { QQ Vir }\end{array}$ & $\begin{array}{l}\text { HS 2201+2610 } \\
\text { V* V391 Peg }^{*} \text { V391 }\end{array}$ & $\begin{array}{r}\text { EC 09582-1137 } \\
\text { PG 0958-116 }\end{array}$ \\
\hline minimum mass & 0.45 & 0.45 & 0.45 & 0.45 & 0.45 \\
\hline $\operatorname{mass}\left[M_{\odot}\right]$ & 0.50 & 0.50 & 0.50 & 0.50 & 0.50 \\
\hline maximum mass & 0.55 & 0.55 & 0.55 & 0.55 & 0.55 \\
\hline minimum radius & 0.209 & 0.149 & 0.132 & 0.198 & 0.134 \\
\hline radius $\left[R_{\odot}\right]$ & 0.247 & 0.186 & 0.146 & 0.234 & 0.147 \\
\hline maximum radius & 0.291 & 0.232 & 0.160 & 0.275 & 0.162 \\
\hline minimum luminosity & 23.4 & 23.1 & 23.0 & 24.2 & 23.0 \\
\hline luminosity $\left[L_{\odot}\right]$ & 35.7 & 40.3 & 28.7 & 36.1 & 28.6 \\
\hline maximum luminosity & 53.7 & 70.8 & 35.5 & 53.5 & 35.5 \\
\hline minimum abs. bol. magnitude & 1.31 & 1.32 & 1.33 & 1.27 & 1.33 \\
\hline abs. bol. magnitude & 0.85 & 0.72 & 1.08 & 0.84 & 1.09 \\
\hline maximum abs. bol. magnitude & 0.39 & 0.11 & 0.85 & 0.41 & 0.85 \\
\hline minimum bol. correction & -2.65 & -3.19 & -3.47 & -2.78 & -3.30 \\
\hline bol. correction & -2.70 & -3.30 & -3.47 & -2.88 & -3.30 \\
\hline maximum bol. correction & -2.77 & -3.32 & -3.53 & -2.92 & -3.38 \\
\hline minimum abs. vis. magnitude & 4.08 & 4.64 & 4.86 & 4.19 & 4.71 \\
\hline abs. vis. magnitude & 3.55 & 4.02 & 4.55 & 3.71 & 4.39 \\
\hline maximum abs. vis. magnitude & 3.16 & 3.31 & 4.32 & 3.19 & 4.15 \\
\hline minimum distance & 1600 & 1419 & 679 & 1191 & 1288 \\
\hline distance $[\mathrm{pc}]$ & 2042 & 1894 & 784 & 1482 & 1494 \\
\hline maximum distance & 2443 & 2618 & 870 & 1888 & 1667 \\
\hline
\end{tabular}


We additionally investigate the contribution of hypothetical G5, K5, M0 and M5 stellar companions to the sdBs fluxes of our targets HS 0444+0458 and HS 0702+6043 in Figure 3.5. We also use blackbody radiations to approximate the spectra of the hypothetical companions but suggest to use model spectra of cool stellar atmospheres instead if a more realistic behavior of the spectral energy distribution, especially in the infra-red, is desired. Given the JHK colors and the errors in the distance determination, we may clearly exclude G- and K-type companions. However, for later types (late M-dwarfs as well as L- and T-dwarfs) their theoretical contribution becomes smaller than the uncertainties in the colors. We can therefore only place an upper limit on any hypothetical companion and state that we can firmly exclude all types earlier than M0.

As an additional remark, Reed \& Stiening (2004) conducted a search for main sequence companions to subdwarf B stars using the Two Micron All Sky Survey (2MASS) and count all those sdBs with $\mathrm{J}-\mathrm{H} \leq 0.065$ as single stars. The J-H colors of the EXOTIME targets are $-0.123,0.043$, $-0.142,0.004$ and -0.243 , respectively, for HS 0702+6043, HS 0444+0458, PG 1325+101, HS 2201+2610 and EC 09582-1137. Applying the above criterion, all our EXOTIME targets would be defined as single stars.

\subsubsection{Hybrid pulsations}

HS $0702+6043$ and HS 2201+2610 are two of only a handful known hybrid sdB pulsators, i.e. they exhibit both short period $p$-modes and long period $g$-modes. The detection of the long periodic intensity variations in the above targets (which are of the order of one hour) also demand some considerations concerning binarity. Schuh et al. (2006) and Lutz et al. (2009) conclude the long periodic variations in HS 0702+6043 and HS 2201+2610, respectively, to be of pulsational origin (i.e. gravity modes) but also discuss hypothetical binary scenarios:

In the case of HS 0702+6043, from the uncontaminated optical spectrum Schuh et al. (2006) exclude a potential F- or G-type main sequence companion as the origin of the low-frequency variation. Additionally, the Roche geometry would not allow to place a MS companion at a supposed one hour orbit. The authors also state that all known close sdB+MS systems show substantial reflection effects, with peak-to-peak amplitudes as large as 0.3 

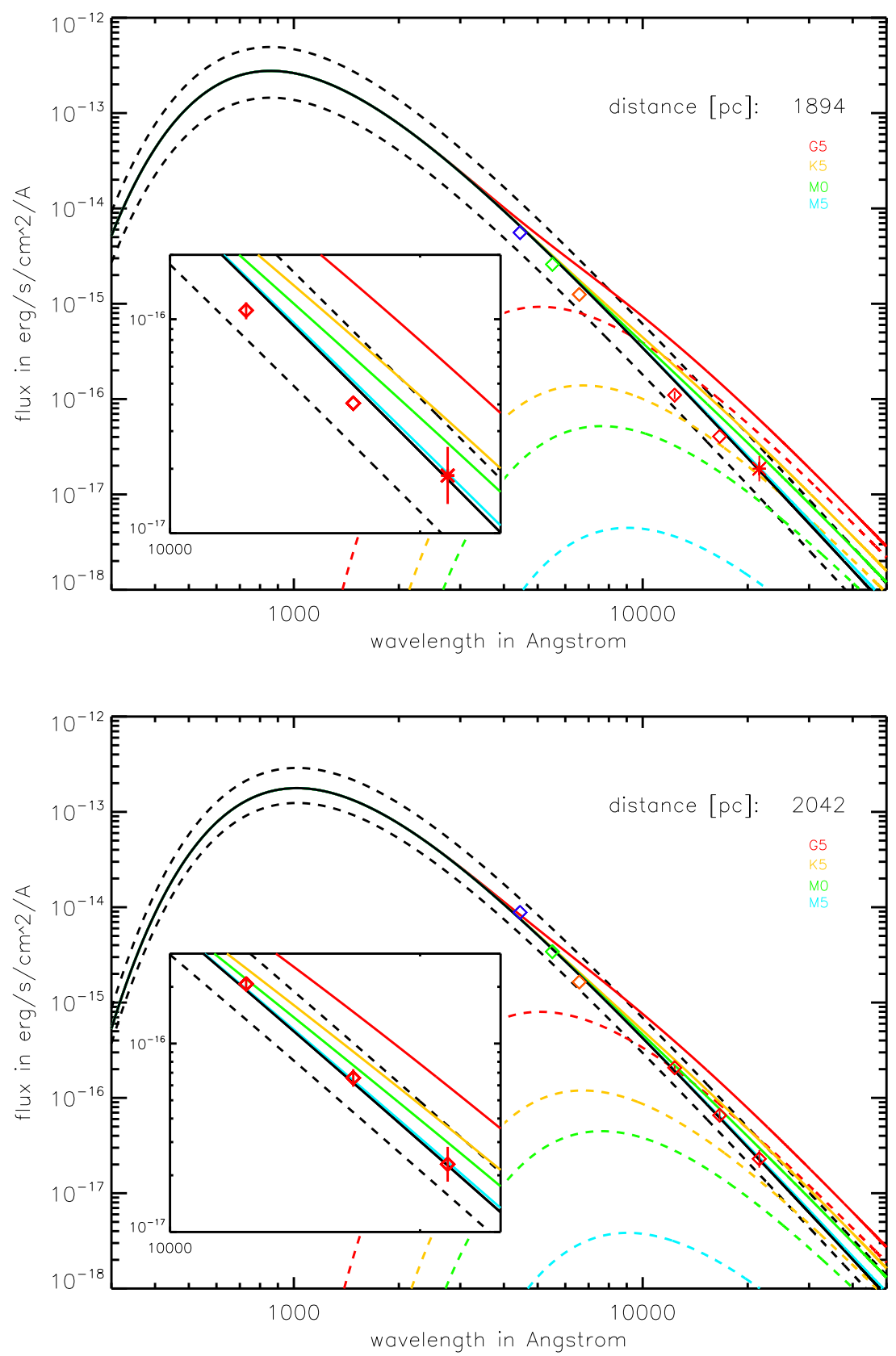

Figure 3.5: Blackbody radiation for HS 0444+0458 (top) and HS 0702+6043 (bottom) compared to BVRJHK colors. Pure contributions of hypothetical main sequence stellar companions of G5 (dashed red line), K5 (dashed orange line), M0 (dashed green line) and M5 (dashed light blue line) type are also displayed as well as the $\mathrm{sdB}+\mathrm{G} 5$ (solid red line), sdB +K5 (solid orange line), sdB +M0 (solid green line) and sdB+M5 (solid light blue line) fluxes. The inset window is a zoom into the infrared JHK region. 
magnitudes. Even in a system with a very low amplitude of 0.083 mag, the variation is still 22 times larger than the variation seen in HS 0702+6043. Schuh et al. (2006) therefore exclude a MS companion as origin for the lowfrequency variation. The presence of a compact companion, resulting in an ellipsoidal deformation of the sdB and an ellipsoidal variation (the orbital period would in that case be twice the intensity variation) seems unlikely. Known sdB+WD systems showing an ellipsoidal effect, have intensity variations of roughly 0.013 to 0.015 magnitudes which is still a factor 3-4 larger than the measured variation in HS 0702+6043. Also, a closer examination of the long periodic variation suggests a multi-periodic character which speaks against binary orbital motion and is a strong indicator for a pulsational origin. Considering these aspects, Schuh et al. (2006) conclude the longperiodic variation to be of pulsational origin with the characteristics of a $g$-mode.

Similar arguments also apply to HS 2201+2610. Lutz et al. (2009) interpret the long-periodic variation in HS $2201+2610$ as a $g$-mode. A binary origin is unlikely for the following reasons: An orbital period in the order of an hour would be at the extreme low limit of the observed and theoretical distributions of orbital periods and, furthermore, a low-mass MS companion as source of the long-period variation can be excluded by geometrical considerations (Roche-limits versus separation in Keplerian orbits). The same holds for sdB+WD configurations down to very low WD masses (below 0.2 Solar masses). A signal caused by ellipsoidal variation would still be ten times larger than the measured variation, even down to very low inclinations below ten degree. Given these arguments, Lutz et al. (2009) conclude the long-periodic variation in HS $2201+2610$ to be of pulsational origin with the characteristics of a $g$-mode. This interpretation is further confirmed by new high quality data obtained at WHT and TNG (Silvotti, priv.comm. and Fig. 1 in Silvotti et al., 2010).

The aspects of hypothetical close stellar companions will be addressed again in section 5.7 when we consider the sensitivity of the timing method. 


\section{Chapter 4}

\section{Data}

The following chapter will give an overview of the data gathered within the EXOTIME project. Among these, the data for two objects, HS $0444+0458$ and HS 0702+6043, are the fundament for the course of this thesis. As previously mentioned, the methods applied here require a huge fundament of regularly taken data. This circumstance will become evident in the following chapters. It is also obvious that the huge amount of data like we need it for our purpose, cannot be provided by a single observing site. The multi-site character of the EXOTIME program was already described in the last chapter. Here, I will describe in more detail the process of organizing multi-site observations and introduce the participating telescope sites. A special focus will be set on the MONET/N telescope. Finally, a collection of each observing night with a detailed description will be given in tabular form. The focus will be set on the two objects analyzed in this thesis: HS $0444+0458$ and HS 0702+6043. For the sake of completeness, the data archive will also contain the remaining EXOTIME targets HS 2201+2610, PG 1325+101 and EC 09582-1137.

The timing method that we will apply in our analysis implies certain requirements and conditions to the photometric time series data:

To derive a single point for the $\mathrm{O}-\mathrm{C}$ diagrams of our selected targets, observations during on average at least three to four consecutive nights with at least two to three hours per target per night are required. The minimum timebase of three nights for each block is needed to provide a sufficient frequency resolution to resolve the pulsational frequencies and to determine the frequencies accurately (see e.g. Figure C.1). At least three hours per 
night are needed for each object to sample enough pulsational cycles. Since we look at low-amplitude, short-period $p$-modes (some mmag on a timescale of about five minutes or less) we require both a short sampling time of less than about 30s and very high signal to noise during these short exposures to detect the low amplitudes. These observational constraints are absolutely crucial for our analysis and are therefore a major determining factor on how we design and schedule our observations.

\subsection{Telescopes}

All observing sites used for gathering the data for this thesis are located on the northern hemisphere. Their locations are marked in Figure 4.1, which also shows pictures of the corresponding telescopes used. The mirror sizes are of small- to medium class, ranging from $0.50 \mathrm{~m}$ up to $3.60 \mathrm{~m}$. Four telescopes are in the USA: Steward Bok $(2.20 \mathrm{~m})$ and Mt.Bigelow Kuiper $(1.56 \mathrm{~m})$ at Steward Observatory, LOAO $(1.00 \mathrm{~m})$ at Mount Lemmon Observatory and MONET/N $(1.20 \mathrm{~m})$ at McDonald Observatory. The other telescopes are located in Europe: NOT $(2.56 \mathrm{~m})$ and TNG $(3.60 \mathrm{~m})$ at Roque de los Muchachos Observatory on La Palma and CA $(2.20 \mathrm{~m})$ at Calar Alto Observatory in Spain. The german sites are Goe $(0.50 \mathrm{~m})$ and Tue $(0.80 \mathrm{~m})$ at the Universities of Göttingen and Tübingen, respectively. Further sites are the italian Loi $(1.52 \mathrm{~m})$ at Loiano Observatory and Asi $(1.82 \mathrm{~m})$ at Asiago Observatory. Finally, there is the hungarian Kon $(1.00 \mathrm{~m})$ at Konkoly Observatory and the lithuanian $\mathrm{Mol}(1.60 \mathrm{~m})$ at Moletai Astronomical Observatory. The telescope shortcuts are also given in Table 4.1 and I refer to the Tables in section 4.2 for further specifications of the single telescopes, settings, filters, detectors etc.

The total contribution of each site to our photometric time-series data archive, as well as the temporal distribution is listed in Figure 4.2. Only the data for the objects HS $0702+6043$ and HS $0444+0458$ are shown. Additionally, the temporal distribution of data according to the specific observation sites is given in Figures 4.3 and 4.4 for HS $0444+0458$ and HS 0702+6043, respectively. 


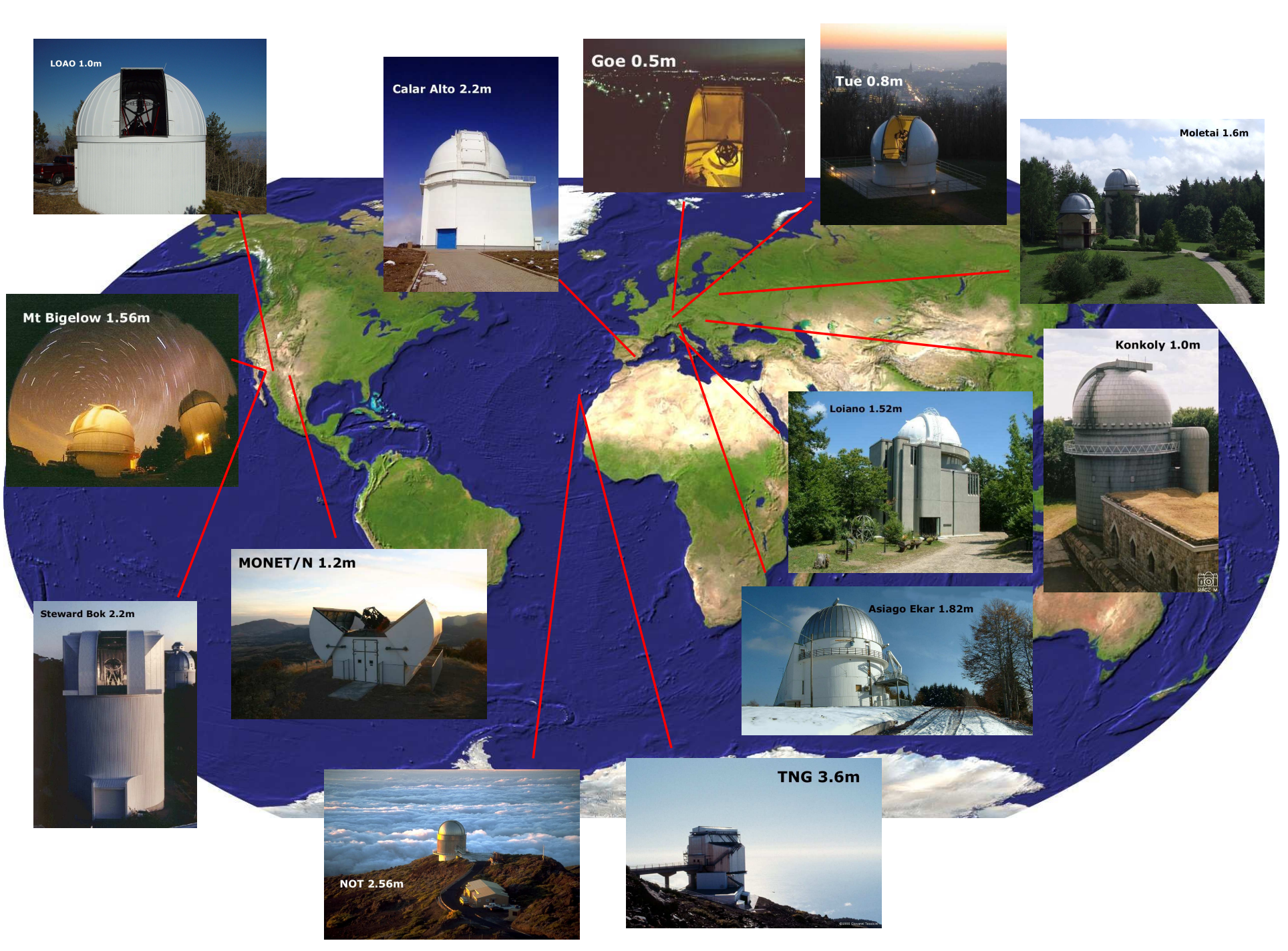

Figure 4.1: Telescope sites used for the EXOTIME program. Red lines show the locations on the world map. Background image: http://www.primap.com/en/html/maps_-_world.html. 


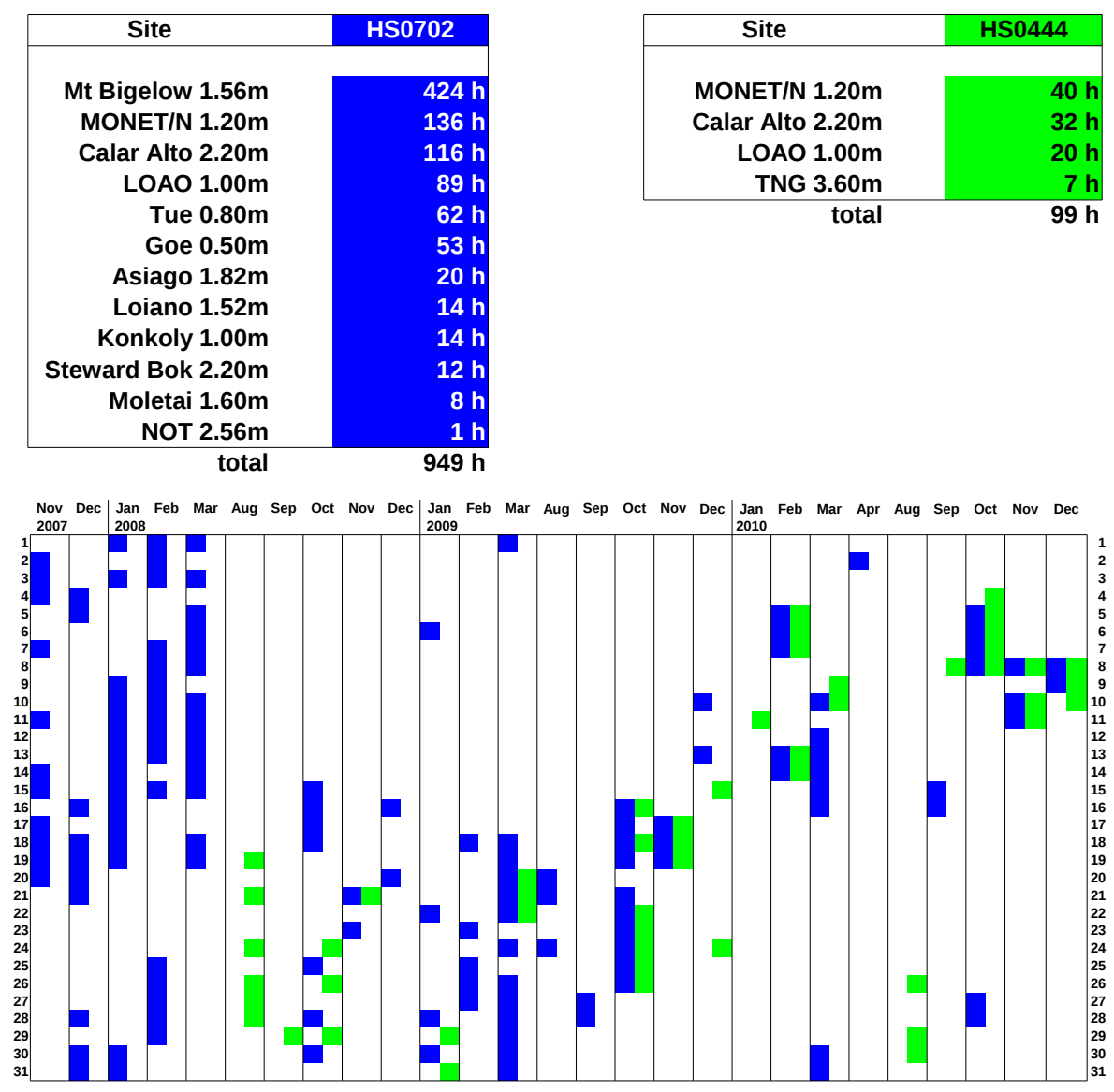

Figure 4.2: Telescope contributions (top) and overall temporal distribution (bottom) for the EXOTIME photometric data of HS0702+6043 (blue) and HS $0444+0458$ (green).

On-site observations are either carried out in visitor mode or in service mode by the local staff (e.g. Calar Alto). Another case is the remote operation of the telescope via browser, as it is possible for e.g. the MONET/N telescope (see following section).

For most of the sites the usual proposal procedure is necessary, resulting in a limited amount of observing time per year or per semester. This is particularly true for the larger telescopes where the chance of over-booking becomes larger.

However, for some sites mentioned above, we have guaranteed access for larger amounts of time: In particular, the huge Mt.Bigelow Kuiper data set on HS $0702+6043$ was subject of another campaign but was generously provided (E.M.Green, priv.comm.) for the inclusion to our EXOTIME data archive and for the specific usage for our purposes. Another very important 


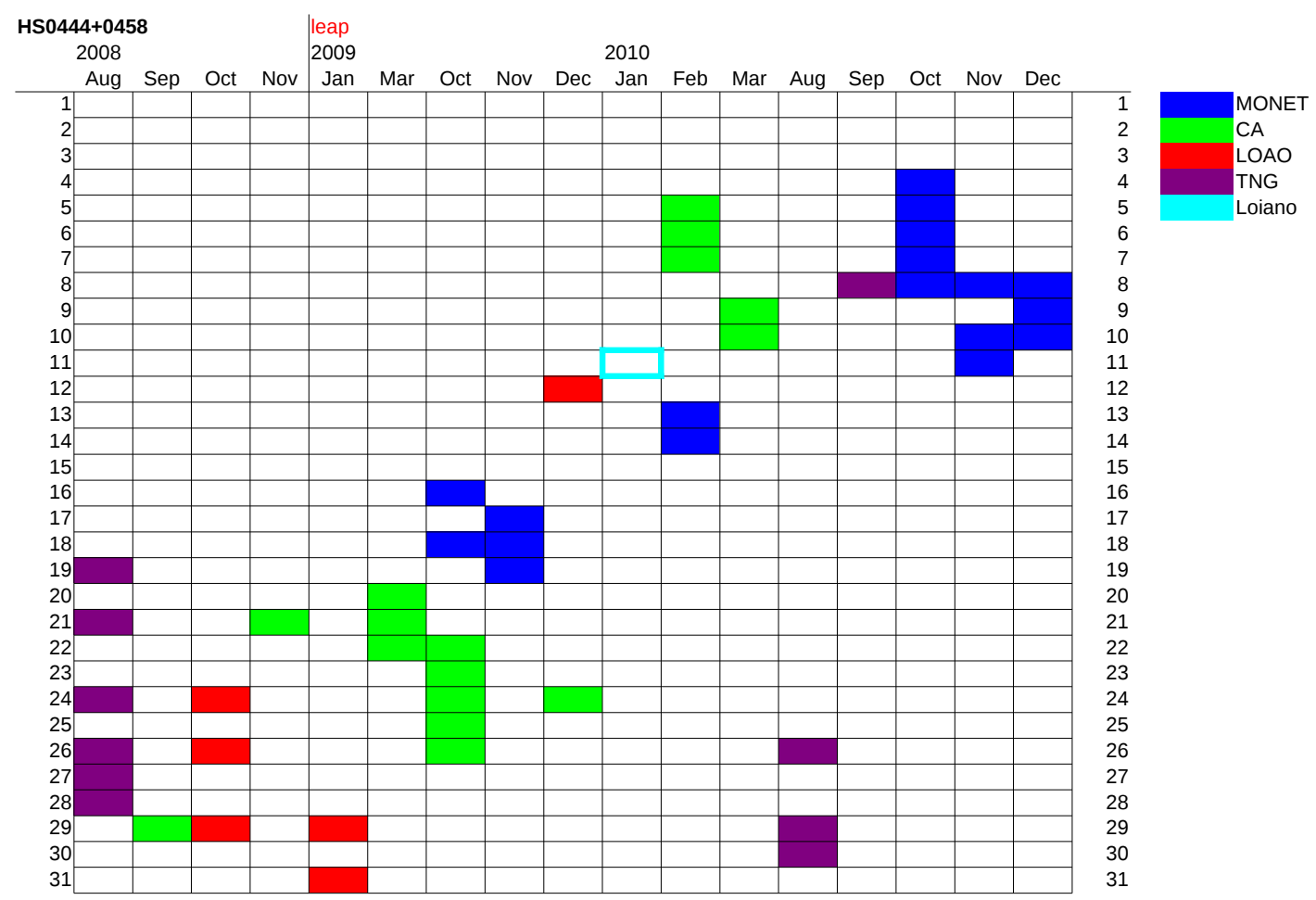

Figure 4.3: Temporal distribution of the HS $0444+0458$ data, color coded according to the observing site.

backbone in our data archive is provided by the MONET/N telescope, because we have a large amount of guaranteed time since it is mainly operated and maintained by the Institute for Astrophysics of the University of Göttingen. Apart from the stand-alone Mt.Bigelow campaign, MONET/N is the biggest contributor to the EXOTIME data for both, HS 0444+0458 and HS $0702+6043$. The following section is intended to provide a closer view on the MONET/N telescope and some of its specifications.

\subsubsection{The MONET/N telescope}

Since a large amount of EXOTIME observations was (and will be) done with the MONET/N telescope, I want to introduce this facility in more detail. All MONET/N contributions to EXOTIME are summarized in Figure 4.5. MONET $^{1}$ (MOnitoring NEtwork of Telescopes) is a network of two $1.2 \mathrm{~m}$ telescopes operated by the Georg-August-Universität Göttingen, the Mc-

${ }^{1}$ http://monet.uni-sw.gwdg.de/foswiki/ 

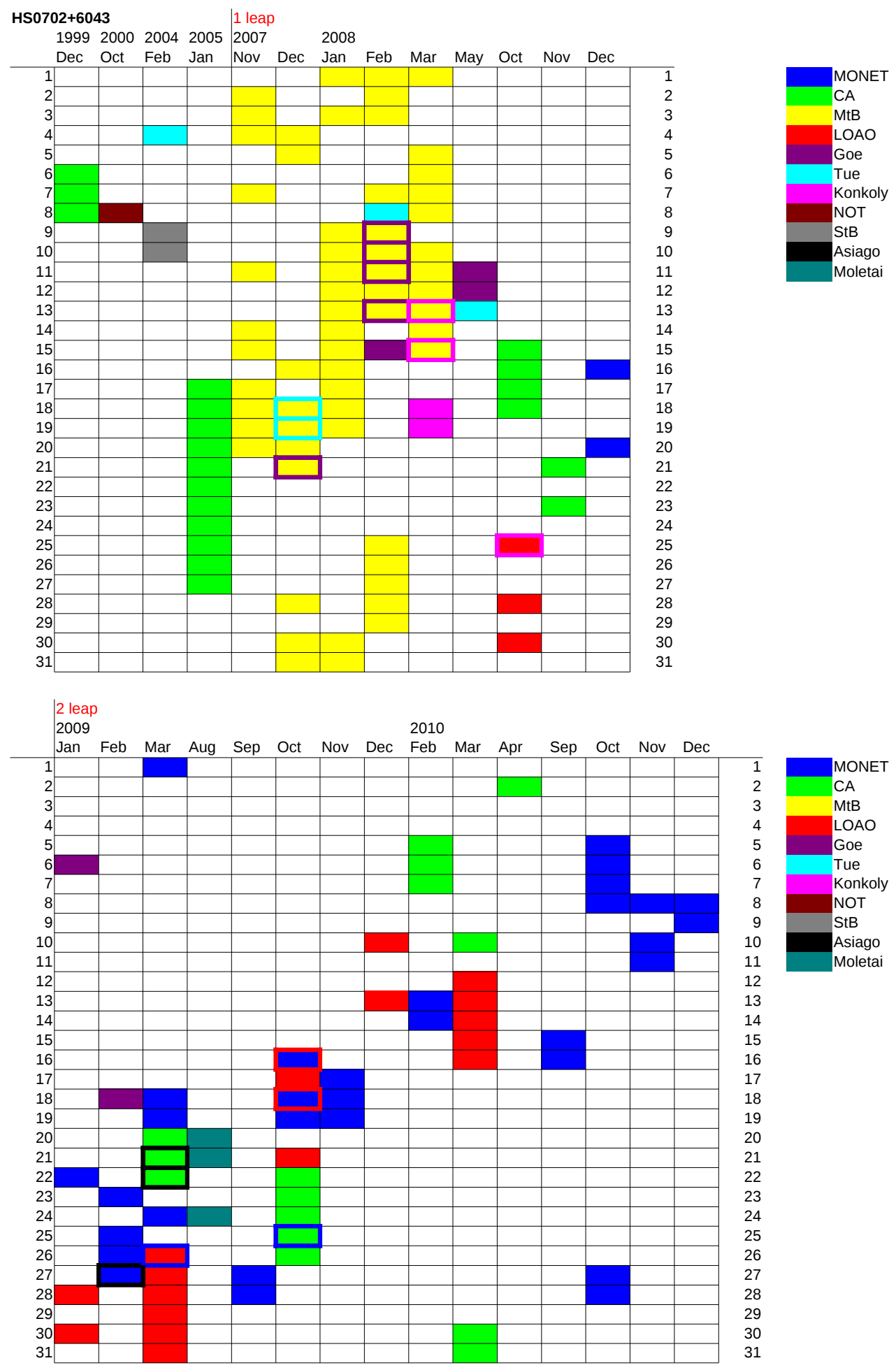

Figure 4.4: Temporal distribution of the HS 0702+6043 data, color coded according to the observing site. 


\section{\begin{tabular}{l|l|l|l} 
HS0702 & HS0444 & HS2201 & PG1325
\end{tabular}}

\begin{tabular}{|c|c|c|c|c|c|c|}
\hline \multirow[t]{2}{*}{2008} & Dec & 16 & 3.0h & & & \\
\hline & & 20 & $2.7 \mathrm{~h}$ & & & \\
\hline 2009 & Jan & 22 & 3.7h & & & \\
\hline \multirow[t]{4}{*}{2009} & Feb & 23 & $3.6 \mathrm{~h}$ & & & \\
\hline & & 25 & $6.4 \mathrm{~h}$ & & & $2.6 \mathrm{~h}$ \\
\hline & & 26 & $6.5 \mathrm{~h}$ & & & $2.4 \mathrm{~h}$ \\
\hline & & 27 & $6.6 \mathrm{~h}$ & & & $3.3 h$ \\
\hline \multirow[t]{5}{*}{2009} & Mar & 1 & $6.2 \mathrm{~h}$ & & & $3.3 \mathrm{~h}$ \\
\hline & & 18 & $3.3 h$ & & & $1.7 \mathrm{~h}$ \\
\hline & & 19 & $3.6 \mathrm{~h}$ & & & \\
\hline & & 24 & $3.4 \mathrm{~h}$ & & & \\
\hline & & 26 & $2.8 \mathrm{~h}$ & & & \\
\hline \multicolumn{3}{|c|}{ SUMMER GAP } & & MMER & & \\
\hline \multirow[t]{3}{*}{2009} & Aug & 18 & & & $3.9 h$ & \\
\hline & & 19 & & & 3.7h & \\
\hline & & 21 & & & $2.8 \mathrm{~h}$ & \\
\hline \multirow[t]{4}{*}{2009} & Sep & 27 & 3.9h & & $1.9 h$ & \\
\hline & & 28 & $4.0 \mathrm{~h}$ & & $2.0 \mathrm{~h}$ & \\
\hline & & 29 & & & $1.6 \mathrm{~h}$ & \\
\hline & & 30 & & & $2.5 \mathrm{~h}$ & \\
\hline \multirow[t]{5}{*}{2009} & Oct & 16 & $4.0 \mathrm{~h}$ & $1.3 \mathrm{~h}$ & $3.2 \mathrm{~h}$ & \\
\hline & & 17 & & & $2.9 \mathrm{~h}$ & \\
\hline & & 18 & $0.9 h$ & $1.6 \mathrm{~h}$ & 4.1h & \\
\hline & & 19 & $6.1 \mathrm{~h}$ & & 4.3h & \\
\hline & & 25 & $2.8 \mathrm{~h}$ & & & \\
\hline \multirow[t]{3}{*}{2009} & Nov & 17 & $5.7 \mathrm{~h}$ & $2.7 \mathrm{~h}$ & $2.4 \mathrm{~h}$ & \\
\hline & & 18 & $5.6 \mathrm{~h}$ & $2.8 \mathrm{~h}$ & $2.9 \mathrm{~h}$ & \\
\hline & & 19 & $5.2 \mathrm{~h}$ & $1.3 \mathrm{~h}$ & $2.5 \mathrm{~h}$ & \\
\hline \multicolumn{3}{|c|}{ SUMMER GAP } & & MMER & & \\
\hline \multirow[t]{2}{*}{2010} & Feb & 13 & 4.0h & $3.2 \mathrm{~h}$ & & \\
\hline & & 14 & $3.9 h$ & $3.0 \mathrm{~h}$ & & $3.2 \mathrm{~h}$ \\
\hline \multirow[t]{2}{*}{2010} & Sep & 15 & $1.5 \mathrm{~h}$ & & & \\
\hline & & 16 & $2.8 h$ & & & \\
\hline \multirow[t]{7}{*}{2010} & Oct & 4 & & $1.5 \mathrm{~h}$ & & \\
\hline & & 5 & $3.4 \mathrm{~h}$ & $1.4 \mathrm{~h}$ & & \\
\hline & & 6 & $2.9 h$ & $2.1 \mathrm{~h}$ & & \\
\hline & & 7 & $2.0 \mathrm{~h}$ & $2.3 h$ & & \\
\hline & & 8 & $2.5 \mathrm{~h}$ & $2.3 \mathrm{~h}$ & & \\
\hline & & 27 & $3.8 \mathrm{~h}$ & & & \\
\hline & & 28 & $1.2 \mathrm{~h}$ & & & \\
\hline \multirow[t]{3}{*}{2010} & Nov & 8 & 3.4h & $2.6 \mathrm{~h}$ & & \\
\hline & & 10 & $3.3 h$ & $2.6 \mathrm{~h}$ & & \\
\hline & & 11 & $2.3 h$ & $1.9 \mathrm{~h}$ & & \\
\hline \multirow[t]{3}{*}{2010} & Dec & 8 & $4.6 h$ & $2.4 \mathrm{~h}$ & & \\
\hline & & 9 & $4.6 h$ & $2.1 \mathrm{~h}$ & & \\
\hline & & 10 & & $3.1 \mathrm{~h}$ & & \\
\hline
\end{tabular}

Figure 4.5: MONET/N photometric contributions for the EXOTIME targets HS 0702+6043 (blue), HS 0444+0458 (green), HS 2201+2610 (red) and PG 1325+101 (yellow). The time on target in each of the nights is given in hours. 
Donald Observatory of the University of Texas, USA, and the South African Astronomical Observatory (Hessman, 2001; Bischoff et al., 2006).

It is funded by the "Astronomie \& Internet" program of the Alfried Krupp von Bohlen und Halbach Foundation. A large amount of the data presented in this thesis was acquired using the MONET site in Texas on the northern hemisphere, hence MONET/North or MONET/N. Operations can be carried out remotely from the home institutions, or from anywhere via a web-browser interface. The primary mirror has a diameter of $1.20 \mathrm{~m}$ and it is currently equipped with an Apogee $1 \mathrm{kx} 1 \mathrm{k}$ E2V CCD camera. The readout times are approximately ten seconds in full frame mode and three seconds with two by two binning. In order to keep the cycle time as short as possible to sample the short $p$-mode oscillations properly, all images were taken in $2 \times 2$ binning and the exposure times were set to $20 \mathrm{~s}$ for the target HS $0444+0458$ and $25 \mathrm{~s}$ for the target HS 0702+6043. All images were taken with a Johnson-Bessel blue filter with a central wavelength of $440 \mathrm{~nm}$.

Unfortunately, due to some major roof mechanism problems, MONET/N experienced various longer down-times (especially in 2008, another one in the first half of 2010 and the last one happened to be in mid 2011) where no operations had been possible. Also, referring to Figure 3.1, one has to keep in mind that the targets are not visible throughout the whole year. The two targets of this thesis are best visible during the winter, therefore our photometric data on these objects are accumulating mainly in the time span October to March.

From the observer's point of view there exists a very nice tool written specifically for MONET by T.-O. Husser to make remote observing much more comfortable. This package is called Claude (Husser, 2011) and provides e.g. automatic focussing along with several other handy features like visibility plots, flatfield tool, positioning and automatic download of images, just to mention a few.

\subsection{Data archive}

The following Tables 4.3 to 4.13 provide a complete collection of detailed information on each observing night. $L$ gives the length of the observation in hours, $N$ the number of exposures, $\sigma_{\text {res }}$ the standard deviation, Exp the 
exposure duration in seconds, Site the telescope name, Inst. and Filter the used setup and Observer the person conducting or being responsible for the observations. The shortcuts for the observing site and the responsible observer are specified in Tables 4.1 and 4.2, respectively.

Data for HS $0702+6043$ can be found in Tables 4.3 to 4.7 and for the target HS 0444+0458 in Tables 4.8 and 4.9. The data archives concerning these two objects, for which I am responsible within the EXOTIME program, are complete up to the end of year 2010.

In addition, the other EXOTIME targets are also listed but in these cases the data may possibly be not complete. For the complete data archives on these targets I refer to the people responsible for their analysis within the EXOTIME program and to the observation schedule which is located at

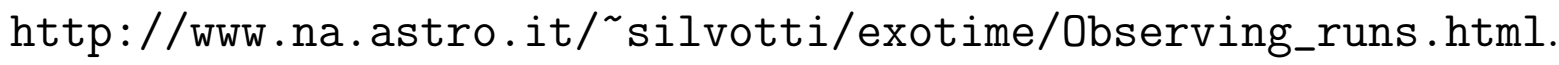
Here, I only give further details for those data which I have at hand personally. For HS 2201+2610 (Table 4.10) this are data after 2006 and only from MONET/N and Calar Alto. For PG 1325+101 (Tables 4.11 and 4.12) and EC 09582-1137 (Table 4.13) also earlier observations, which were not part of the EXOTIME program, are listed.

Table 4.1: Telescope site shortcuts for Tables 4.3- 4.13,

\begin{tabular}{cc}
\hline Shortcut & Site \\
\hline Asi & Asiago 182cm Copernico Telescope \\
CA & Calar Alto Observatory \\
Goe & Göttingen IAG 50cm Telescope \\
Kon & Konkoly RCC Telescope \\
LOAO & Mt. Lemmon Optical Astronomy Observatory \\
Loi & Loiano 152cm Telescope \\
Mol & Moletai 1.6m Telescope \\
M/N & MONET/North Telescope \\
MtB & Mt. Bigelow Kuiper Telescope \\
NOT & Nordic Optical Telescope \\
StB & Steward Observatory Bok Telescope \\
TNG & Telescopio Nazionale Galileo \\
Tue & Tübingen 80cm Telescope \\
\hline
\end{tabular}


Table 4.2: Observer shortcuts for Tables 4.3 - 4.13 ,

\begin{tabular}{cc|cc}
\hline Shortcut & Observer & Shortcut & Observer \\
\hline AA & Alberto Aguirre & RL & Ronny Lutz \\
ADB & Antonio De Blasi & RØ & Roy Østensen \\
AH & Agnes Hoffmann & RS & Roberto Silvotti \\
BB & Benjamin Beeck & SB & Serena Benatti \\
BL & Björn Loeptien & SD & Stefan Dreizler \\
EMG & Elizabeth M. Green & SL & Silvio Leccia \\
FH & Felipe Hoyo & SLK & Seung-Lee Kim \\
FHo & Felix Hormuth & SS & Sonja Schuh \\
LM & Luzma Montoya & SW & Sascha Werhahn \\
LN & Lisa Nortmann & TN & Thorsten Nagel \\
MA & Manuel Alises & TOH & Tim-Oliver Husser \\
MH & Markus Hundertmark & TS & Thorsten Stahn \\
MP & Margit Paparó & US & Ulf Seemann \\
PP & Péter Pápic & ZB & Zsófia Bognár \\
RC & Riccardo Claudi & & \\
RK & Renate Kruspe & & \\
RJ & Rimas Janulis & & \\
\hline
\end{tabular}


Table 4.3: Photometric data archive for HS0702+6043, part a.

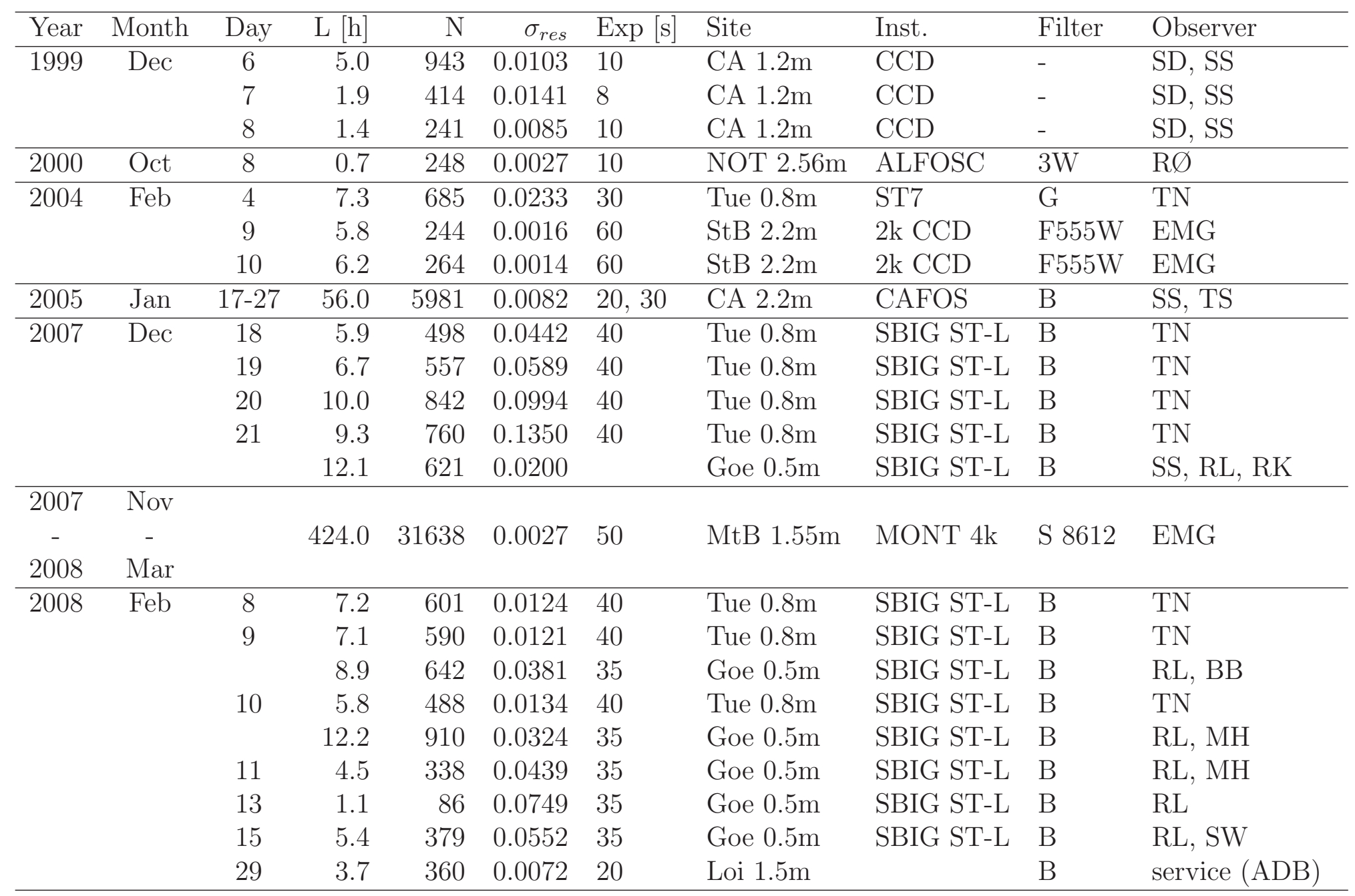


Table 4.4: Photometric data archive for HS0702+6043, part b.

\begin{tabular}{|c|c|c|c|c|c|c|c|c|c|c|}
\hline Year & Month & Day & $\mathrm{L}[\mathrm{h}]$ & $\mathrm{N}$ & $\sigma_{\text {res }}$ & $\operatorname{Exp}[\mathrm{s}]$ & Site & Inst. & Filter & Observer \\
\hline \multirow[t]{23}{*}{2008} & \multirow[t]{8}{*}{ Mar } & 1 & 5.7 & 451 & 0.0065 & 25 & Loi $1.5 \mathrm{~m}$ & & B & service (ADB) \\
\hline & & 2 & 5.1 & 429 & 0.0108 & 25 & Loi $1.5 \mathrm{~m}$ & & B & service (ADB) \\
\hline & & 5 & 0.6 & 47 & 0.0238 & 35 & Goe $0.5 \mathrm{~m}$ & SBIG ST-L & B & RL, SS \\
\hline & & 13 & 3.7 & 126 & 0.0069 & 30 & Asi 1.8 & Afosc & $\mathrm{R}$ & $\mathrm{SB}, \mathrm{RC}$ \\
\hline & & & 2.3 & 248 & 0.0105 & 30 & Kon 1.0m & PI VA 1300B & B & $\mathrm{MP}, \mathrm{PP}$ \\
\hline & & 15 & 4.6 & 597 & 0.0095 & 25 & Kon $1.0 \mathrm{~m}$ & PI VA 1300B & B & MP, PP \\
\hline & & 18 & 3.7 & 348 & 0.0111 & 35 & Kon $1.0 \mathrm{~m}$ & PI VA 1300B & B & $\mathrm{MP}, \mathrm{PP}$ \\
\hline & & 19 & 1.1 & 137 & 0.0088 & 25 & Kon $1.0 \mathrm{~m}$ & PI VA 1300B & B & $\mathrm{MP}, \mathrm{PP}$ \\
\hline & \multirow[t]{3}{*}{ May } & 11 & 3.0 & 204 & 0.0315 & 40 & Goe $0.5 \mathrm{~m}$ & SBIG ST-L & B & SW \\
\hline & & 12 & 1.2 & 160 & 0.0380 & 40 & Goe $0.5 \mathrm{~m}$ & SBIG ST-L & B & SW \\
\hline & & 13 & 3.2 & 266 & 0.0254 & 40 & Tue $0.8 \mathrm{~m}$ & SBIG ST-L & B & $\mathrm{AH}$ \\
\hline & \multirow[t]{8}{*}{ Oct } & 15 & 1.3 & 113 & 0.0085 & 10 & $\mathrm{CA} 2.2 \mathrm{~m}$ & CAFOS & B & service (LM) \\
\hline & & 16 & 0.8 & 65 & 0.0101 & 10 & CA $2.2 \mathrm{~m}$ & CAFOS & B & service (MA) \\
\hline & & 17 & 0.5 & 48 & 0.0152 & 10 & $\mathrm{CA} 2.2 \mathrm{~m}$ & CAFOS & B & service (MA) \\
\hline & & 18 & 1.6 & 143 & 0.0068 & 10 & $\mathrm{CA} 2.2 \mathrm{~m}$ & CAFOS & B & service (MA) \\
\hline & & 25 & 2.6 & 285 & 0.0089 & 30 & Kon $1.0 \mathrm{~m}$ & PI VA 1300B & B & $\mathrm{ZB}$ \\
\hline & & & 4.4 & 518 & 0.0107 & 15 & LOAO $1.0 \mathrm{~m}$ & $2 \mathrm{k}$ CCD & B & SLK \\
\hline & & 28 & 2.9 & 326 & 0.0104 & 15 & LOAO $1.0 \mathrm{~m}$ & $2 \mathrm{k}$ CCD & B & SLK \\
\hline & & 30 & 3.9 & 455 & 0.0104 & 15 & LOAO $1.0 \mathrm{~m}$ & 2k CCD & B & SLK \\
\hline & \multirow[t]{2}{*}{ Nov } & 21 & 3.0 & 254 & 0.0048 & 10 & $\mathrm{CA} 2.2 \mathrm{~m}$ & CAFOS & B & service (MA) \\
\hline & & 23 & 5.7 & 496 & 0.0064 & 10 & $\mathrm{CA} 2.2 \mathrm{~m}$ & CAFOS & B & service (MA) \\
\hline & \multirow[t]{2}{*}{ Dec } & 16 & 3.0 & 243 & 0.0068 & 25 & $\mathrm{M} / \mathrm{N} 1.2 \mathrm{~m}$ & $1 \mathrm{k} C \mathrm{CD}$ & B & $\mathrm{RL}, \mathrm{BL}$ \\
\hline & & 20 & 2.7 & 202 & 0.0116 & 25 & $\mathrm{M} / \mathrm{N} 1.2 \mathrm{~m}$ & 1k CCD & B & $\mathrm{RL}$ \\
\hline \multirow[t]{4}{*}{2009} & \multirow[t]{4}{*}{ Jan } & 6 & 1.6 & 143 & 0.0364 & 30 & Goe $0.5 \mathrm{~m}$ & SBIG ST-L & B & RL, US \\
\hline & & 22 & 3.7 & 334 & 0.0080 & 25 & $\mathrm{M} / \mathrm{N} 1.2 \mathrm{~m}$ & 1k CCD & B & $\mathrm{RL}$ \\
\hline & & 28 & 5.4 & 473 & 0.0147 & 20 & LOAO $1.0 \mathrm{~m}$ & $2 \mathrm{k}$ CCD & B & SLK \\
\hline & & 30 & 6.6 & 662 & 0.0138 & 20 & LOAO $1.0 \mathrm{~m}$ & $2 \mathrm{k} C C D$ & B & SLK \\
\hline
\end{tabular}


Table 4.5: Photometric data archive for HS0702+6043, part c.

\begin{tabular}{|c|c|c|c|c|c|c|c|c|c|c|}
\hline Year & Month & Day & $\mathrm{L}[\mathrm{h}]$ & $\mathrm{N}$ & $\sigma_{\text {res }}$ & $\operatorname{Exp}[\mathrm{s}]$ & Site & Inst. & Filter & Observer \\
\hline \multirow[t]{27}{*}{2009} & Feb & 18 & 2.4 & 202 & 0.0256 & 30 & Goe $0.5 \mathrm{~m}$ & SBIG ST-L & B & RL, TOH \\
\hline & & 23 & 3.6 & 304 & 0.0126 & 25 & $\mathrm{M} / \mathrm{N} 1.2 \mathrm{~m}$ & 1k CCD & B & RL, US, MH \\
\hline & & 25 & 6.4 & 564 & 0.0076 & 25 & $\mathrm{M} / \mathrm{N} 1.2 \mathrm{~m}$ & $1 \mathrm{k} C \mathrm{CD}$ & B & $\mathrm{RL}$ \\
\hline & & 26 & 6.5 & 536 & 0.0111 & 25 & $\mathrm{M} / \mathrm{N} 1.2 \mathrm{~m}$ & $1 \mathrm{k} C \mathrm{CD}$ & B & RL, LN \\
\hline & & 27 & 6.6 & 556 & 0.0083 & 25 & $\mathrm{M} / \mathrm{N} 1.2 \mathrm{~m}$ & $1 \mathrm{k} C \mathrm{CD}$ & B & $\mathrm{RL}$ \\
\hline & & & 5.7 & 532 & 0.0067 & 15 & Asi $1.8 \mathrm{~m}$ & Afosc & no & $\mathrm{SB}, \mathrm{RC}$ \\
\hline & Mar & 01 & 6.2 & 497 & 0.0090 & 25 & $\mathrm{M} / \mathrm{N} 1.2 \mathrm{~m}$ & $1 \mathrm{k} C \mathrm{CD}$ & B & $\mathrm{RL}$ \\
\hline & & 18 & 3.3 & 279 & 0.0060 & 25 & $\mathrm{M} / \mathrm{N} 1.2 \mathrm{~m}$ & $1 \mathrm{k} C \mathrm{CD}$ & B & $\mathrm{RL}$ \\
\hline & & 19 & 3.6 & 291 & 0.0098 & 25 & $\mathrm{M} / \mathrm{N} 1.2 \mathrm{~m}$ & $1 \mathrm{k} C \mathrm{CD}$ & B & RL \\
\hline & & 20 & 2.4 & 243 & 0.0058 & 10 & CA $2.2 \mathrm{~m}$ & CAFOS & B & service (MA) \\
\hline & & 21 & 2.4 & 232 & 0.0068 & 10 & CA $2.2 \mathrm{~m}$ & CAFOS & B & service (MA) \\
\hline & & & 6.0 & 351 & 0.0150 & 30 & Asi $1.8 \mathrm{~m}$ & Afosc & B & $\mathrm{SB}, \mathrm{RC}$ \\
\hline & & 22 & 2.4 & 245 & 0.0049 & 10 & $\mathrm{CA} 2.2 \mathrm{~m}$ & CAFOS & B & service (MA) \\
\hline & & & 4.9 & 279 & 0.0140 & 30 & Asi $1.8 \mathrm{~m}$ & Afosc & B & $\mathrm{SB}, \mathrm{RC}$ \\
\hline & & 24 & 3.4 & 243 & 0.0092 & 25 & $\mathrm{M} / \mathrm{N} 1.2 \mathrm{~m}$ & $1 \mathrm{k} C \mathrm{CD}$ & B & $\mathrm{RL}$ \\
\hline & & 26 & 2.8 & 251 & 0.0081 & 25 & $\mathrm{M} / \mathrm{N} 1.2 \mathrm{~m}$ & $1 \mathrm{k} C \mathrm{CD}$ & B & RL, US \\
\hline & & & 3.9 & 396 & 0.0118 & 20 & LOAO $1.0 \mathrm{~m}$ & 2k CCD & B & SLK \\
\hline & & 27 & 1.3 & 160 & 0.0185 & 20 & LOAO $1.0 \mathrm{~m}$ & 2k CCD & B & SLK \\
\hline & & 28 & 3.9 & 404 & 0.0172 & 20 & LOAO $1.0 \mathrm{~m}$ & $2 \mathrm{k}$ CCD & B & SLK \\
\hline & & 29 & 2.9 & 309 & 0.0112 & 20 & LOAO $1.0 \mathrm{~m}$ & $2 \mathrm{k}$ CCD & B & SLK \\
\hline & & 30 & 3.8 & 386 & 0.0144 & 20 & LOAO $1.0 \mathrm{~m}$ & 2k CCD & B & SLK \\
\hline & & 31 & 4.0 & 399 & 0.0154 & 20 & LOAO $1.0 \mathrm{~m}$ & $2 \mathrm{k}$ CCD & B & SLK \\
\hline & Aug & 20 & 2.1 & 384 & 0.0058 & 20 & Mol 1.6m & & & RJ \\
\hline & & 21 & 3.4 & 605 & 0.0065 & 20 & Mol 1.6m & & & RJ \\
\hline & & 24 & 2.8 & 473 & 0.0061 & 20 & Mol 1.6m & & & RJ \\
\hline & Sep & 27 & 3.9 & 406 & 0.0132 & 25 & $\mathrm{M} / \mathrm{N} 1.2 \mathrm{~m}$ & 1k CCD & B & $\mathrm{RL}$ \\
\hline & & 28 & 4.0 & 420 & 0.0117 & 25 & $\mathrm{M} / \mathrm{N} 1.2 \mathrm{~m}$ & $1 \mathrm{k} C \mathrm{CD}$ & B & RL \\
\hline
\end{tabular}


Table 4.6: Photometric data archive for HS0702+6043, part d.

\begin{tabular}{|c|c|c|c|c|c|c|c|c|c|c|}
\hline Year & Month & Day & $\mathrm{L}[\mathrm{h}]$ & $\mathrm{N}$ & $\sigma_{\text {res }}$ & $\operatorname{Exp}[\mathrm{s}]$ & Site & Inst. & Filter & Observer \\
\hline \multirow[t]{18}{*}{2009} & Oct & 16 & 3.0 & 238 & 0.0120 & 20 & LOAO $1.0 \mathrm{~m}$ & 2k CCD & B & SLK \\
\hline & & & 4.0 & 412 & 0.0099 & 25 & $\mathrm{M} / \mathrm{N} 1.2 \mathrm{~m}$ & $1 \mathrm{k} C \mathrm{CD}$ & B & $\mathrm{RL}$ \\
\hline & & 17 & 4.0 & 493 & 0.0141 & 20 & LOAO $1.0 \mathrm{~m}$ & $2 \mathrm{k} C \mathrm{CD}$ & B & SLK \\
\hline & & 18 & 4.8 & 594 & 0.0149 & 20 & LOAO $1.0 \mathrm{~m}$ & $2 \mathrm{k}$ CCD & B & SLK \\
\hline & & & 0.9 & 100 & 0.0109 & 25 & $\mathrm{M} / \mathrm{N} 1.2 \mathrm{~m}$ & 1k CCD & B & RL \\
\hline & & 19 & 6.1 & 645 & 0.0119 & 25 & $\mathrm{M} / \mathrm{N} 1.2 \mathrm{~m}$ & 1k CCD & B & $\mathrm{RL}$ \\
\hline & & 21 & 1.5 & 179 & 0.0151 & 20 & LOAO $1.0 \mathrm{~m}$ & 2k CCD & B & SLK \\
\hline & & 22 & 3.9 & 359 & 0.0076 & 10 & CA $2.2 \mathrm{~m}$ & CAFOS & B & service (AA) \\
\hline & & 23 & 3.6 & 334 & 0.0067 & 10 & $\mathrm{CA} 2.2 \mathrm{~m}$ & CAFOS & B & service (MA) \\
\hline & & 24 & 3.1 & 289 & 0.0061 & 10 & CA $2.2 \mathrm{~m}$ & CAFOS & B & service (MA) \\
\hline & & 25 & 2.8 & 297 & 0.0082 & 25 & $\mathrm{M} / \mathrm{N} 1.2 \mathrm{~m}$ & $1 \mathrm{k} C \mathrm{CD}$ & B & $\mathrm{RL}$ \\
\hline & & & 3.4 & 315 & 0.0078 & 10 & CA $2.2 \mathrm{~m}$ & CAFOS & B & service (MA) \\
\hline & & 26 & 3.0 & 279 & 0.0064 & 10 & CA $2.2 \mathrm{~m}$ & CAFOS & B & service (MA) \\
\hline & Nov & 17 & 5.7 & 562 & 0.0082 & 25 & $\mathrm{M} / \mathrm{N} 1.2 \mathrm{~m}$ & $1 \mathrm{k} C \mathrm{CD}$ & B & $\mathrm{RL}$ \\
\hline & & 18 & 5.6 & 585 & 0.0089 & 25 & $\mathrm{M} / \mathrm{N} 1.2 \mathrm{~m}$ & 1k CCD & B & RL \\
\hline & & 19 & 5.2 & 543 & 0.0113 & 25 & $\mathrm{M} / \mathrm{N} 1.2 \mathrm{~m}$ & $1 \mathrm{k} C \mathrm{CD}$ & B & RL \\
\hline & Dec & 10 & 5.0 & 587 & 0.0142 & 20 & LOAO $1.0 \mathrm{~m}$ & $2 \mathrm{k} C C D$ & B & SLK \\
\hline & & 13 & 2.0 & 240 & 0.0169 & 20 & LOAO $1.0 \mathrm{~m}$ & $2 \mathrm{k}$ CCD & B & SLK \\
\hline \multirow[t]{5}{*}{2010} & Feb & 5 & 2.6 & 238 & 0.0071 & 10 & CA $2.2 \mathrm{~m}$ & CAFOS & B & service (LM) \\
\hline & & 6 & 2.8 & 258 & 0.0071 & 10 & $\mathrm{CA} 2.2 \mathrm{~m}$ & CAFOS & B & service (FHo) \\
\hline & & 7 & 1.8 & 146 & 0.0119 & 10 & CA $2.2 \mathrm{~m}$ & CAFOS & B & service $(\mathrm{FHo})$ \\
\hline & & 13 & 4.0 & 422 & 0.0099 & 25 & $\mathrm{M} / \mathrm{N} 1.2 \mathrm{~m}$ & $1 \mathrm{k} C \mathrm{CD}$ & B & $\mathrm{RL}$ \\
\hline & & 14 & 3.9 & 416 & 0.0091 & 25 & $\mathrm{M} / \mathrm{N} 1.2 \mathrm{~m}$ & 1k CCD & B & RL \\
\hline
\end{tabular}


Table 4.7: Photometric data archive for HS0702+6043, part e.

\begin{tabular}{|c|c|c|c|c|c|c|c|c|c|c|}
\hline Year & Month & Day & $\mathrm{L}[\mathrm{h}]$ & $\mathrm{N}$ & $\sigma_{r e s}$ & $\operatorname{Exp}[\mathrm{s}]$ & Site & Inst. & Filter & Observer \\
\hline \multirow[t]{22}{*}{2010} & \multirow[t]{8}{*}{ Mar } & 10 & 3.1 & 288 & 0.0086 & 10 & CA $2.2 \mathrm{~m}$ & CAFOS & B & service (MA) \\
\hline & & 12 & 4.9 & 594 & 0.0131 & 20 & LOAO $1.0 \mathrm{~m}$ & $2 \mathrm{k} C \mathrm{CD}$ & B & SLK \\
\hline & & 13 & 5.0 & 610 & 0.0131 & 20 & LOAO $1.0 \mathrm{~m}$ & $2 \mathrm{k} C \mathrm{CD}$ & B & SLK \\
\hline & & 14 & 5.1 & 538 & 0.0131 & 20 & LOAO $1.0 \mathrm{~m}$ & $2 \mathrm{k}$ CCD & B & SLK \\
\hline & & 15 & 5.2 & 597 & 0.0136 & 20 & LOAO $1.0 \mathrm{~m}$ & $2 \mathrm{k}$ CCD & B & SLK \\
\hline & & 16 & 5.2 & 570 & 0.0192 & 20 & LOAO $1.0 \mathrm{~m}$ & $2 \mathrm{k}$ CCD & B & SLK \\
\hline & & 30 & 2.3 & 215 & 0.0137 & 10 & $\mathrm{CA} 2.2 \mathrm{~m}$ & CAFOS & B & service $(\mathrm{FH})$ \\
\hline & & 31 & 1.7 & 158 & 0.0131 & 10 & $\mathrm{CA} 2.2 \mathrm{~m}$ & CAFOS & B & service $(\mathrm{FH})$ \\
\hline & Apr & 2 & 0.8 & 74 & 0.0110 & 10 & CA $2.2 \mathrm{~m}$ & CAFOS & B & service $(\mathrm{FH})$ \\
\hline & \multirow{2}{*}{ Sep } & 15 & 1.5 & 160 & 0.0090 & 25 & $\mathrm{M} / \mathrm{N} 1.2 \mathrm{~m}$ & $1 \mathrm{k}$ CCD & B & RL \\
\hline & & 16 & 2.8 & 363 & 0.0144 & 25 & $\mathrm{M} / \mathrm{N} 1.2 \mathrm{~m}$ & $1 \mathrm{k}$ CCD & B & $\mathrm{RL}$ \\
\hline & \multirow[t]{6}{*}{ Oct } & 5 & 3.4 & 380 & 0.0210 & 25 & $\mathrm{M} / \mathrm{N} 1.2 \mathrm{~m}$ & $1 \mathrm{k}$ CCD & B & RL \\
\hline & & 6 & 2.9 & 355 & 0.0099 & 25 & $\mathrm{M} / \mathrm{N} 1.2 \mathrm{~m}$ & $1 \mathrm{k}$ CCD & B & RL \\
\hline & & 7 & 2.0 & 242 & 0.0075 & 25 & $\mathrm{M} / \mathrm{N} 1.2 \mathrm{~m}$ & $1 \mathrm{k}$ CCD & B & RL \\
\hline & & 8 & 2.5 & 322 & 0.0104 & 25 & $\mathrm{M} / \mathrm{N} 1.2 \mathrm{~m}$ & $1 \mathrm{k}$ CCD & B & $\mathrm{RL}$ \\
\hline & & 27 & 3.8 & 470 & 0.0190 & 25 & $\mathrm{M} / \mathrm{N} 1.2 \mathrm{~m}$ & $1 \mathrm{k} C \mathrm{CD}$ & B & RL \\
\hline & & 28 & 1.2 & 149 & 0.0126 & 25 & $\mathrm{M} / \mathrm{N} 1.2 \mathrm{~m}$ & $1 \mathrm{k}$ CCD & B & RL \\
\hline & \multirow[t]{3}{*}{ Nov } & 8 & 3.4 & 424 & 0.0105 & 25 & $\mathrm{M} / \mathrm{N} 1.2 \mathrm{~m}$ & $1 \mathrm{k}$ CCD & B & RL \\
\hline & & 10 & 3.3 & 410 & 0.0109 & 25 & $\mathrm{M} / \mathrm{N} 1.2 \mathrm{~m}$ & $1 \mathrm{k}$ CCD & B & RL \\
\hline & & 11 & 2.3 & 297 & 0.0100 & 25 & $\mathrm{M} / \mathrm{N} 1.2 \mathrm{~m}$ & $1 \mathrm{k} C \mathrm{CD}$ & B & RL \\
\hline & \multirow[t]{2}{*}{ Dec } & 8 & 4.6 & 555 & 0.0125 & 25 & $\mathrm{M} / \mathrm{N} 1.2 \mathrm{~m}$ & $1 \mathrm{k}$ CCD & B & RL \\
\hline & & 9 & 4.6 & 577 & 0.0135 & 25 & $\mathrm{M} / \mathrm{N} 1.2 \mathrm{~m}$ & $1 \mathrm{k}$ CCD & B & $\mathrm{RL}$ \\
\hline
\end{tabular}


Table 4.8: Photometric data archive for HS0444+0458, part a.

\begin{tabular}{|c|c|c|c|c|c|c|c|c|c|c|}
\hline Year & Month & Day & $\mathrm{L}[\mathrm{h}]$ & $\mathrm{N}$ & $\sigma_{\text {res }}$ & $\operatorname{Exp}[\mathrm{s}]$ & Site & Inst. & Filter & Observer \\
\hline \multirow[t]{11}{*}{2008} & \multirow[t]{6}{*}{ Aug } & 19 & 0.5 & 107 & 0.0069 & 5 & TNG $3.6 \mathrm{~m}$ & DOLORES & B & RS, SL \\
\hline & & 21 & 1.0 & 237 & 0.0076 & 5 & TNG 3.6m & DOLORES & B & RS, SL \\
\hline & & 24 & 0.7 & 173 & 0.0060 & 5 & TNG 3.6m & DOLORES & B & RS, SL \\
\hline & & 26 & 1.0 & 224 & 0.0051 & 5 & TNG $3.6 \mathrm{~m}$ & DOLORES & B & RS, SL \\
\hline & & 27 & 2.1 & 357 & 0.0040 & 10 & TNG $3.6 \mathrm{~m}$ & DOLORES & B & $\mathrm{RS}, \mathrm{SL}$ \\
\hline & & 28 & 1.7 & 230 & 0.0042 & 10 & TNG 3.6m & DOLORES & B & $\mathrm{RS}, \mathrm{SL}$ \\
\hline & Sep & 29 & 2.2 & 171 & 0.0089 & 10 & CA $2.2 \mathrm{~m}$ & CAFOS & B & service $(\mathrm{FH})$ \\
\hline & \multirow[t]{3}{*}{ Oct } & 24 & 2.8 & 300 & 0.0191 & 12 & LOAO $1.0 \mathrm{~m}$ & $2 \mathrm{k}$ CCD & B & SLK \\
\hline & & 26 & 4.9 & 588 & 0.0232 & 12 & LOAO $1.0 \mathrm{~m}$ & $2 \mathrm{k} C C D$ & B & SLK \\
\hline & & 29 & 1.2 & 163 & 0.0175 & 12 & LOAO $1.0 \mathrm{~m}$ & $2 \mathrm{k}$ CCD & B & SLK \\
\hline & Nov & 21 & 3.2 & 263 & 0.0092 & 10 & CA $2.2 \mathrm{~m}$ & CAFOS & B & service (MA) \\
\hline \multirow[t]{17}{*}{2009} & \multirow[t]{2}{*}{ Jan } & 29 & 2.6 & 275 & 0.0239 & 20 & LOAO $1.0 \mathrm{~m}$ & $2 \mathrm{k}$ CCD & B & SLK \\
\hline & & 31 & 4.7 & 497 & 0.0275 & 20 & LOAO $1.0 \mathrm{~m}$ & $2 \mathrm{k}$ CCD & B & SLK \\
\hline & \multirow[t]{3}{*}{ Mar } & 20 & 1.0 & 94 & 0.0090 & 10 & $\mathrm{CA} 2.2 \mathrm{~m}$ & CAFOS & B & service (MA) \\
\hline & & 21 & 1.3 & 135 & 0.0121 & 10 & $\mathrm{CA} 2.2 \mathrm{~m}$ & CAFOS & B & service (MA) \\
\hline & & 22 & 1.2 & 124 & 0.0099 & 10 & $\mathrm{CA} 2.2 \mathrm{~m}$ & CAFOS & B & service (MA) \\
\hline & \multirow[t]{7}{*}{ Oct } & 16 & 1.3 & 195 & 0.0138 & 20 & $\mathrm{M} / \mathrm{N} 1.2 \mathrm{~m}$ & $1 \mathrm{k} C \mathrm{CD}$ & B & RL \\
\hline & & 18 & 1.6 & 242 & 0.0148 & 20 & $\mathrm{M} / \mathrm{N} 1.2 \mathrm{~m}$ & $1 \mathrm{k} C \mathrm{CD}$ & B & $\mathrm{RL}$ \\
\hline & & 22 & 2.1 & 235 & 0.0102 & 10 & CA $2.2 \mathrm{~m}$ & CAFOS & B & service (AA) \\
\hline & & 23 & 2.9 & 330 & 0.0082 & 10 & $\mathrm{CA} 2.2 \mathrm{~m}$ & CAFOS & B & service (MA) \\
\hline & & 24 & 2.2 & 244 & 0.0075 & 10 & $\mathrm{CA} 2.2 \mathrm{~m}$ & CAFOS & B & service (MA) \\
\hline & & 25 & 1.7 & 104 & 0.0061 & 10 & $\mathrm{CA} 2.2 \mathrm{~m}$ & CAFOS & B & service (MA) \\
\hline & & 26 & 3.4 & 387 & 0.0086 & 10 & CA $2.2 \mathrm{~m}$ & CAFOS & B & service (MA) \\
\hline & \multirow[t]{3}{*}{ Nov } & 17 & 2.7 & 422 & 0.0160 & 20 & $\mathrm{M} / \mathrm{N} 1.2 \mathrm{~m}$ & $1 \mathrm{k} C \mathrm{CD}$ & B & $\mathrm{RL}$ \\
\hline & & 18 & 2.8 & 438 & 0.0155 & 20 & $\mathrm{M} / \mathrm{N} 1.2 \mathrm{~m}$ & $1 \mathrm{k} C \mathrm{CD}$ & B & RL \\
\hline & & 19 & 1.3 & 153 & 0.0169 & 20 & $\mathrm{M} / \mathrm{N} 1.2 \mathrm{~m}$ & $1 \mathrm{k} C \mathrm{CD}$ & B & RL \\
\hline & \multirow[t]{2}{*}{ Dec } & 15 & 4.5 & 527 & 0.0359 & 20 & LOAO $1.0 \mathrm{~m}$ & $2 \mathrm{k}$ CCD & B & SLK \\
\hline & & 24 & 0.5 & 58 & 0.0206 & 10 & $\mathrm{CA} 2.2 \mathrm{~m}$ & CAFOS & B & service (AA) \\
\hline
\end{tabular}


Table 4.9: Photometric data archive for HS0444+0458, part b.

\begin{tabular}{|c|c|c|c|c|c|c|c|c|c|c|}
\hline Year & Month & Day & $\mathrm{L}[\mathrm{h}]$ & $\mathrm{N}$ & $\sigma_{\text {res }}$ & $\operatorname{Exp}[\mathrm{s}]$ & Site & Inst. & Filter & Observer \\
\hline \multirow[t]{23}{*}{2010} & Jan & 11 & 1.4 & & & & Loi $1.5 \mathrm{~m}$ & BFOSC & $\mathrm{B}$ & $\mathrm{RS}$ \\
\hline & Feb & 5 & 2.8 & 264 & 0.0160 & 10 & $\mathrm{CA} 2.2 \mathrm{~m}$ & CAFOS & B & service (LM) \\
\hline & & 6 & 2.8 & 317 & 0.0113 & 10 & CA $2.2 \mathrm{~m}$ & CAFOS & B & service (FHo) \\
\hline & & 7 & 1.1 & 114 & 0.0106 & 10 & $\mathrm{CA} 2.2 \mathrm{~m}$ & CAFOS & B & service (FHo) \\
\hline & & 13 & 3.2 & 497 & 0.0158 & 20 & $\mathrm{M} / \mathrm{N} 1.2 \mathrm{~m}$ & 1k CCD & B & $\mathrm{RL}$ \\
\hline & & 14 & 3.0 & 404 & 0.0136 & 20 & $\mathrm{M} / \mathrm{N} 1.2 \mathrm{~m}$ & $1 \mathrm{k} C \mathrm{CD}$ & B & $\mathrm{RL}$ \\
\hline & Mar & 9 & 1.3 & 146 & 0.0184 & 10 & CA $2.2 \mathrm{~m}$ & CAFOS & B & service (MA) \\
\hline & & 10 & 2.7 & 298 & 0.0151 & 10 & CA $2.2 \mathrm{~m}$ & CAFOS & B & service (MA) \\
\hline & Aug & 26 & 1.1 & & & & TNG $3.6 \mathrm{~m}$ & & B & $\mathrm{RS}$ \\
\hline & & 29 & 1.1 & & & & TNG $3.6 \mathrm{~m}$ & & B & $\mathrm{RS}$ \\
\hline & & 30 & 1.1 & & & & TNG $3.6 \mathrm{~m}$ & & B & RS \\
\hline & Sep & 8 & 1.1 & & & & TNG $3.6 \mathrm{~m}$ & & B & $\mathrm{RS}$ \\
\hline & Oct & 4 & 1.5 & 223 & 0.0118 & 20 & $\mathrm{M} / \mathrm{N} 1.2 \mathrm{~m}$ & 1k CCD & B & RL, TOH \\
\hline & & 5 & 1.4 & 174 & 0.0263 & 20 & $\mathrm{M} / \mathrm{N} 1.2 \mathrm{~m}$ & $1 \mathrm{k} C \mathrm{CD}$ & B & $\mathrm{RL}$ \\
\hline & & 6 & 2.1 & 328 & 0.0134 & 20 & $\mathrm{M} / \mathrm{N} 1.2 \mathrm{~m}$ & $1 \mathrm{k} C \mathrm{CD}$ & B & RL \\
\hline & & 7 & 2.3 & 350 & 0.0109 & 20 & $\mathrm{M} / \mathrm{N} 1.2 \mathrm{~m}$ & 1k CCD & B & RL \\
\hline & & 8 & 2.3 & 344 & 0.0145 & 20 & $\mathrm{M} / \mathrm{N} 1.2 \mathrm{~m}$ & $1 \mathrm{k} C \mathrm{CD}$ & B & RL \\
\hline & Nov & 8 & 2.6 & 378 & 0.0143 & 20 & $\mathrm{M} / \mathrm{N} 1.2 \mathrm{~m}$ & $1 \mathrm{k} C \mathrm{CD}$ & B & $\mathrm{RL}$ \\
\hline & & 10 & 2.6 & 391 & 0.0155 & 20 & $\mathrm{M} / \mathrm{N} 1.2 \mathrm{~m}$ & $1 \mathrm{k} C \mathrm{CD}$ & B & RL \\
\hline & & 11 & 1.9 & 289 & 0.0122 & 20 & $\mathrm{M} / \mathrm{N} 1.2 \mathrm{~m}$ & 1k CCD & B & RL \\
\hline & Dec & 8 & 2.4 & 373 & 0.0201 & 20 & $\mathrm{M} / \mathrm{N} 1.2 \mathrm{~m}$ & $1 \mathrm{k} C \mathrm{CD}$ & B & RL \\
\hline & & 9 & 2.1 & 311 & 0.0186 & 20 & $\mathrm{M} / \mathrm{N} 1.2 \mathrm{~m}$ & $1 \mathrm{k} C \mathrm{CD}$ & B & RL \\
\hline & & 10 & 3.1 & 389 & 0.0170 & 20 & $\mathrm{M} / \mathrm{N} 1.2 \mathrm{~m}$ & $1 \mathrm{k} C \mathrm{CD}$ & B & RL \\
\hline
\end{tabular}


Table 4.10: MONET and Calar Alto data archive for HS2201+2610.

\begin{tabular}{|c|c|c|c|c|c|c|c|c|c|c|}
\hline Year & Month & Day & $\mathrm{L}[\mathrm{h}]$ & $\mathrm{N}$ & $\sigma_{\text {res }}$ & $\operatorname{Exp}[\mathrm{s}]$ & Site & Inst. & Filter & Observer \\
\hline \multirow[t]{4}{*}{2006} & Sep & 19,20 & & & & & $\mathrm{M} / \mathrm{N} 1.2 \mathrm{~m}$ & & $\mathrm{~B}$ & \\
\hline & & $24,26,27,28$ & & & & & $\mathrm{CA} 2.2 \mathrm{~m}$ & & $\mathrm{~B}$ & \\
\hline & Nov & 18 & & & & & $\mathrm{CA} 2.2 \mathrm{~m}$ & & B & \\
\hline & & 22,24 & & & & & $\mathrm{M} / \mathrm{N} 1.2 \mathrm{~m}$ & & B & \\
\hline 2007 & May & $10-14,22,23$ & & & & & $\mathrm{M} / \mathrm{N} 1.2 \mathrm{~m}$ & & B & \\
\hline \multirow[t]{2}{*}{2008} & Sep & 20,23 & & & & & $\mathrm{CA} 2.2 \mathrm{~m}$ & & B & service (AA) \\
\hline & Oct & 16 & & & & & $\mathrm{CA} 2.2 \mathrm{~m}$ & & B & \\
\hline \multirow[t]{21}{*}{2009} & Aug & 18 & 3.9 & 383 & & 25 & $\mathrm{M} / \mathrm{N} 1.2 \mathrm{~m}$ & $1 \mathrm{k} C \mathrm{CD}$ & B & $\mathrm{RL}$ \\
\hline & & $19 \mathrm{a}$ & 2.2 & 210 & & 25 & $\mathrm{M} / \mathrm{N} 1.2 \mathrm{~m}$ & $1 \mathrm{k} C \mathrm{CD}$ & B & $\mathrm{RL}$ \\
\hline & & $19 \mathrm{~b}$ & 1.5 & 155 & & 25 & $\mathrm{M} / \mathrm{N} 1.2 \mathrm{~m}$ & $1 \mathrm{k} C \mathrm{CD}$ & B & RL \\
\hline & & 21 & 2.8 & 244 & & 25 & $\mathrm{M} / \mathrm{N} 1.2 \mathrm{~m}$ & $1 \mathrm{k} C \mathrm{CD}$ & B & RL \\
\hline & Sep & 27 & 1.9 & 200 & & 25 & $\mathrm{M} / \mathrm{N} 1.2 \mathrm{~m}$ & $1 \mathrm{k} C \mathrm{CD}$ & B & $\mathrm{RL}$ \\
\hline & & 28 & 2.0 & 203 & & 25 & $\mathrm{M} / \mathrm{N} 1.2 \mathrm{~m}$ & $1 \mathrm{k} C \mathrm{CD}$ & B & RL \\
\hline & & 29 & 1.6 & 162 & & 25 & $\mathrm{M} / \mathrm{N} 1.2 \mathrm{~m}$ & $1 \mathrm{k} C \mathrm{CD}$ & B & $\mathrm{RL}$ \\
\hline & & 30 & 2.5 & 256 & & 25 & $\mathrm{M} / \mathrm{N} 1.2 \mathrm{~m}$ & $1 \mathrm{k} C \mathrm{CD}$ & B & $\mathrm{RL}$ \\
\hline & Oct & 16 & 3.2 & 323 & & 25 & $\mathrm{M} / \mathrm{N} 1.2 \mathrm{~m}$ & $1 \mathrm{k} C \mathrm{CD}$ & B & $\mathrm{RL}$ \\
\hline & & 17 & 2.9 & 235 & & 25 & $\mathrm{M} / \mathrm{N} 1.2 \mathrm{~m}$ & $1 \mathrm{k} C \mathrm{CD}$ & B & $\mathrm{RL}$ \\
\hline & & 18 & 4.1 & 395 & & 25 & $\mathrm{M} / \mathrm{N} 1.2 \mathrm{~m}$ & $1 \mathrm{k} C \mathrm{CD}$ & $\mathrm{B}$ & $\mathrm{RL}$ \\
\hline & & 19 & 4.3 & 447 & & 25 & $\mathrm{M} / \mathrm{N} 1.2 \mathrm{~m}$ & $1 \mathrm{k} C \mathrm{CD}$ & B & $\mathrm{RL}$ \\
\hline & & 22 & 3.4 & 349 & & 10 & $\mathrm{CA} 2.2 \mathrm{~m}$ & CAFOS & B & service (AA) \\
\hline & & 23 & 2.2 & 250 & & 10 & $\mathrm{CA} 2.2 \mathrm{~m}$ & CAFOS & B & service (MA) \\
\hline & & 24 & 2.1 & 222 & & 10 & $\mathrm{CA} 2.2 \mathrm{~m}$ & CAFOS & B & service (MA) \\
\hline & & 25 & 2.2 & 224 & & 10 & $\mathrm{CA} 2.2 \mathrm{~m}$ & CAFOS & B & service (MA) \\
\hline & & 26 & 4.1 & 468 & & 10 & $\mathrm{CA} 2.2 \mathrm{~m}$ & CAFOS & B & service (MA) \\
\hline & Nov & 17 & 2.4 & 252 & & 25 & $\mathrm{M} / \mathrm{N} 1.2 \mathrm{~m}$ & $1 \mathrm{k} C \mathrm{CD}$ & B & $\mathrm{RL}$ \\
\hline & & 18 & 2.9 & 304 & & 25 & $\mathrm{M} / \mathrm{N} 1.2 \mathrm{~m}$ & $1 \mathrm{k} C \mathrm{CD}$ & B & $\mathrm{RL}$ \\
\hline & & 19 & 2.5 & 260 & & 25 & $\mathrm{M} / \mathrm{N} 1.2 \mathrm{~m}$ & $1 \mathrm{k} C \mathrm{CD}$ & B & $\mathrm{RL}$ \\
\hline & Dec & 24 & 1.7 & 196 & & 10 & $\mathrm{CA} 2.2 \mathrm{~m}$ & CAFOS & $\mathrm{B}$ & service (AA) \\
\hline
\end{tabular}


Table 4.11: Photometric data archive for PG1325+101, part a.

\begin{tabular}{|c|c|c|c|c|c|c|c|c|c|c|}
\hline Year & Month & Day & $\mathrm{L}[\mathrm{h}]$ & $\mathrm{N}$ & $\sigma_{\text {res }}$ & $\operatorname{Exp}[\mathrm{s}]$ & Site & Inst. & Filter & Observer \\
\hline 2003 & Mar-Apr & & $215 \mathrm{~h}$ & & & & multisite & & & $\mathrm{RS}$ \\
\hline \multirow{6}{*}{2008} & Feb-May & & $95.5 \mathrm{~h}$ & & & & multisite & & $\mathrm{V}$ & Ostensen, Baran \\
\hline & Mar & 1,2 & 7.4 & & & & Loi 1.5m & & & $\mathrm{RS}$ \\
\hline & & 13 & 4.8 & & & & Kon $1.0 \mathrm{~m}$ & & & MP \\
\hline & & 14 & 2.7 & & & & Asi 1.8m & & & RC,SB,EC \\
\hline & Jun & 2 & 0.7 & & & & Loi $1.5 \mathrm{~m}$ & & & $\mathrm{RS}$ \\
\hline & & 4,6 & 3.0 & & & & Mol 1.6m & & & RJ \\
\hline \multirow[t]{18}{*}{2009} & Jan & $28-31$ & 9.0 & & & & $\mathrm{LOAO} 1.0 \mathrm{~m}$ & & B & SLK \\
\hline & Feb & 25 & 2.6 & 300 & & 20 & $\mathrm{M} / \mathrm{N} 1.2 \mathrm{~m}$ & 1k CCD & B & $\mathrm{RL}$ \\
\hline & & 26 & 2.4 & 252 & & 20 & $\mathrm{M} / \mathrm{N} 1.2 \mathrm{~m}$ & $1 \mathrm{k} C \mathrm{CD}$ & B & RL \\
\hline & & 27 & 3.3 & 370 & & 20 & $\mathrm{M} / \mathrm{N} 1.2 \mathrm{~m}$ & 1k CCD & B & $\mathrm{RL}$ \\
\hline & & $23-28$ & 2.0 & & & & TT1 1.5m & & & DM,MD,FC \\
\hline & Mar & 01 & 3.3 & 355 & & 20 & $\mathrm{M} / \mathrm{N} 1.2 \mathrm{~m}$ & 1k CCD & B & $\mathrm{RL}$ \\
\hline & & 18 & 1.7 & 192 & & 20 & $\mathrm{M} / \mathrm{N} 1.2 \mathrm{~m}$ & 1k CCD & B & RL \\
\hline & & 20 & 3.9 & 301 & & 10 & $\mathrm{CA} 2.2 \mathrm{~m}$ & & B & service (MA) \\
\hline & & 21 & 2.0 & 155 & & 10 & $\mathrm{CA} 2.2 \mathrm{~m}$ & & B & service (MA) \\
\hline & & 22 & 3.9 & 300 & & 10 & $\mathrm{CA} 2.2 \mathrm{~m}$ & & B & service (MA) \\
\hline & & $28-31$ & 23.8 & & & & LOAO $1.0 \mathrm{~m}$ & & & SLK \\
\hline & Apr & 22 & 2.9 & & & & Loi $1.5 \mathrm{~m}$ & & & IB \\
\hline & & 29 & 2.9 & & & & $\mathrm{CA} 2.2 \mathrm{~m}$ & & B & \\
\hline & May & 6 & 3.9 & & & & $\mathrm{CA} 2.2 \mathrm{~m}$ & & B & \\
\hline & & 25 & 3.9 & & & & LOAO $1.0 \mathrm{~m}$ & & & SLK \\
\hline & & 26 & 5.0 & & & & LOAO $1.0 \mathrm{~m}$ & & & SLK \\
\hline & & 27 & 5.1 & & & & $\mathrm{LOAO} 1.0 \mathrm{~m}$ & & & SLK \\
\hline & Jun & 24 & 1.2 & & & & Loi $1.5 \mathrm{~m}$ & & & IB \\
\hline
\end{tabular}


Table 4.12: Photometric data archive for PG1325+101, part b.

\begin{tabular}{|c|c|c|c|c|c|c|c|c|c|c|}
\hline Year & Month & Day & $\mathrm{L}[\mathrm{h}]$ & $\mathrm{N}$ & $\sigma_{\text {res }}$ & $\operatorname{Exp}[\mathrm{s}]$ & Site & Inst. & Filter & Observer \\
\hline \multirow[t]{9}{*}{2010} & Feb & 5 & 2.4 & & & & CA $2.2 \mathrm{~m}$ & & $\mathrm{~B}$ & \\
\hline & & 6 & 2.3 & & & & $\mathrm{CA} 2.2 \mathrm{~m}$ & & B & \\
\hline & & 14 & 3.2 & 192 & & 20 & $\mathrm{M} / \mathrm{N} 1.2 \mathrm{~m}$ & $1 \mathrm{k} C \mathrm{CD}$ & B & $\mathrm{RL}$ \\
\hline & Mar & 10 & 1.9 & 230 & & 10 & $\mathrm{CA} 2.2 \mathrm{~m}$ & CAFOS & B & service (MA) \\
\hline & & 27 & 1.6 & 199 & & 10 & $\mathrm{CA} 2.2 \mathrm{~m}$ & CAFOS & B & service (MA) \\
\hline & & 28 & 2.3 & 272 & & 10 & $\mathrm{CA} 2.2 \mathrm{~m}$ & CAFOS & B & service (MA) \\
\hline & Apr & 2 & 2.6 & 302 & & 10 & CA $2.2 \mathrm{~m}$ & CAFOS & B & service $(\mathrm{FH})$ \\
\hline & & 3 & 3.5 & 393 & & 10 & CA $2.2 \mathrm{~m}$ & CAFOS & B & service $(\mathrm{FH})$ \\
\hline & & 4 & 2.3 & 276 & & 10 & $\mathrm{CA} 2.2 \mathrm{~m}$ & CAFOS & B & service $(\mathrm{FH})$ \\
\hline
\end{tabular}


Table 4.13: Photometric data archive for EC09582-1137.

\begin{tabular}{|c|c|c|c|c|c|c|c|c|c|c|}
\hline Year & Month & Day & $\mathrm{L}[\mathrm{h}]$ & $\mathrm{N}$ & $\sigma_{\text {res }}$ & $\operatorname{Exp}[\mathrm{s}]$ & Site & Inst. & Filter & Observer \\
\hline 2005 & Feb & $12-15$ & $18.5 \mathrm{~h}$ & & & & & & & Kilkenny \\
\hline \multirow[t]{3}{*}{2006} & Feb & $31,2,3$ & & & & & & & & Kilkenny \\
\hline & Mar & $28,29,30,3$ & & & & & & & & Kilkenny \\
\hline & Apr & 25,26 & & & & & & & & Kilkenny \\
\hline 2008 & Mar & $10-14$ & $30 \mathrm{~h}$ & & & 10 & NTT $3.6 \mathrm{~m}$ & SuSi-2 & no & Randall \\
\hline \multirow[t]{3}{*}{2009} & Jan & 29,31 & & & & & LOAO $1.0 \mathrm{~m}$ & & B & SLK \\
\hline & Mar & 20 & 1.8 & 208 & & 10 & CA $2.2 \mathrm{~m}$ & CAFOS & B & service (MA) \\
\hline & & 22 & 1.8 & 201 & & 10 & CA $2.2 \mathrm{~m}$ & CAFOS & B & service (MA) \\
\hline \multirow[t]{5}{*}{2010} & Feb & 5 & 2.3 & 266 & & 10 & CA $2.2 \mathrm{~m}$ & CAFOS & B & service (LM) \\
\hline & & 6 & 2.3 & 279 & & 10 & $\mathrm{CA} 2.2 \mathrm{~m}$ & CAFOS & B & service (FHo) \\
\hline & Mar & 10 & 2.0 & 240 & & 10 & $\mathrm{CA} 2.2 \mathrm{~m}$ & CAFOS & B & service (MA) \\
\hline & & 30 & 1.4 & 116 & & 10 & CA $2.2 \mathrm{~m}$ & CAFOS & B & service $(\mathrm{FH})$ \\
\hline & & 31 & 2.3 & 278 & & 10 & $\mathrm{CA} 2.2 \mathrm{~m}$ & CAFOS & B & service $(\mathrm{FH})$ \\
\hline
\end{tabular}




\section{Chapter 5}

\section{Methods}

The following chapter is intended to describe the methods required for the analysis. Beginning with the actual observations, the basic steps from retrieving the raw data to constructing the weighted relative light curves will be described as well as the methodology to produce an $\mathrm{O}-\mathrm{C}$ (Observed minus Calculated) diagram. Subsequently, $\mathrm{O}-\mathrm{C}$ related formulas and errors are derived and I will address how to interpret given $\mathrm{O}-\mathrm{C}$ diagrams. Finally, the timing method is put in comparison to various other exoplanet detection methods. I will focus on the timing method explicitly applied to subdwarf B stars.

\subsection{Time-series photometry}

The data needed for doing our analysis is time series relative photometry. For all of the EXOTIME targets, there is at least one stable reference star in the field of view, in most cases more than four. If possible with the respective telescope characteristics of each site, the same reference stars are used throughout the program for each corresponding target.

Since we are dealing with multi-site data, it is absolutely crucial to take special care of consistency of the data. For those sites where we have the raw data at hand, which is true for most of the data, we use the IDL based program TRIPP (Schuh et al., 2003) to carry out standard aperture photometry including bias-, dark-, flat- and extinction corrections (fitting second order polynomials) as well as normalizing the relative light curves. The output of the TRIPP program yields an ASCII table containing time in RJD (Reduced Julian Date, i.e. Julian Date-2400000) and relative in- 
tensity normalized to a mean of unity.

In the cases where we were provided with pre-reduced data tables or with tables containing count information of targets, reference stars and background, we did the relative photometry separately. To ensure consistency, we finally fed the corresponding tables to a TRIPP subroutine for the normalization and extinction correction to produce the same ASCII tables as mentioned above.

At the end of the data reduction, there are the relative normalized light curves given in time (RJD) versus relative intensity (normalized to unity). All observations were in B filters and the times refer to the midpoint of an exposure to ensure consistency between different data sets and in particular between data from different observation sites. We have performed several cross checks to ensure that there are no systematic temporal offsets between data coming from different telescope facilities.

\subsection{Time corrections}

Whenever working with temporal phenomena in astronomy, it is necessary to give accurate information on the time stamps or time formats used in the observations and analysis. The required precision of these time stamps is given depending on the astrophysical problem considered. A time stamp comprises two basic concepts: the reference frame and the time standard. A detailed review is given in Eastman et al. (2010).

- Reference frame:

Measuring the time of light coming from a certain object with the Earth as reference frame (i.e. GJD, Geocentric Julian Date) will introduce an uncertainty of up to 16.6 minutes over the course of a year due to Earth's motion around the Sun and the finite speed of light. This can be corrected by using the Sun's center as reference point, the Heliocentric Julian Date (HJD). However, even the Sun moves due to the gravitational influence of the planets in the Solar system. The induced error is dominated by the influence of Jupiter and Saturn and can be as large as \pm 4 seconds. Therefore, if sub-second accuracy is desired, HJD is not appropriate to use. To also correct for this effect, one uses 


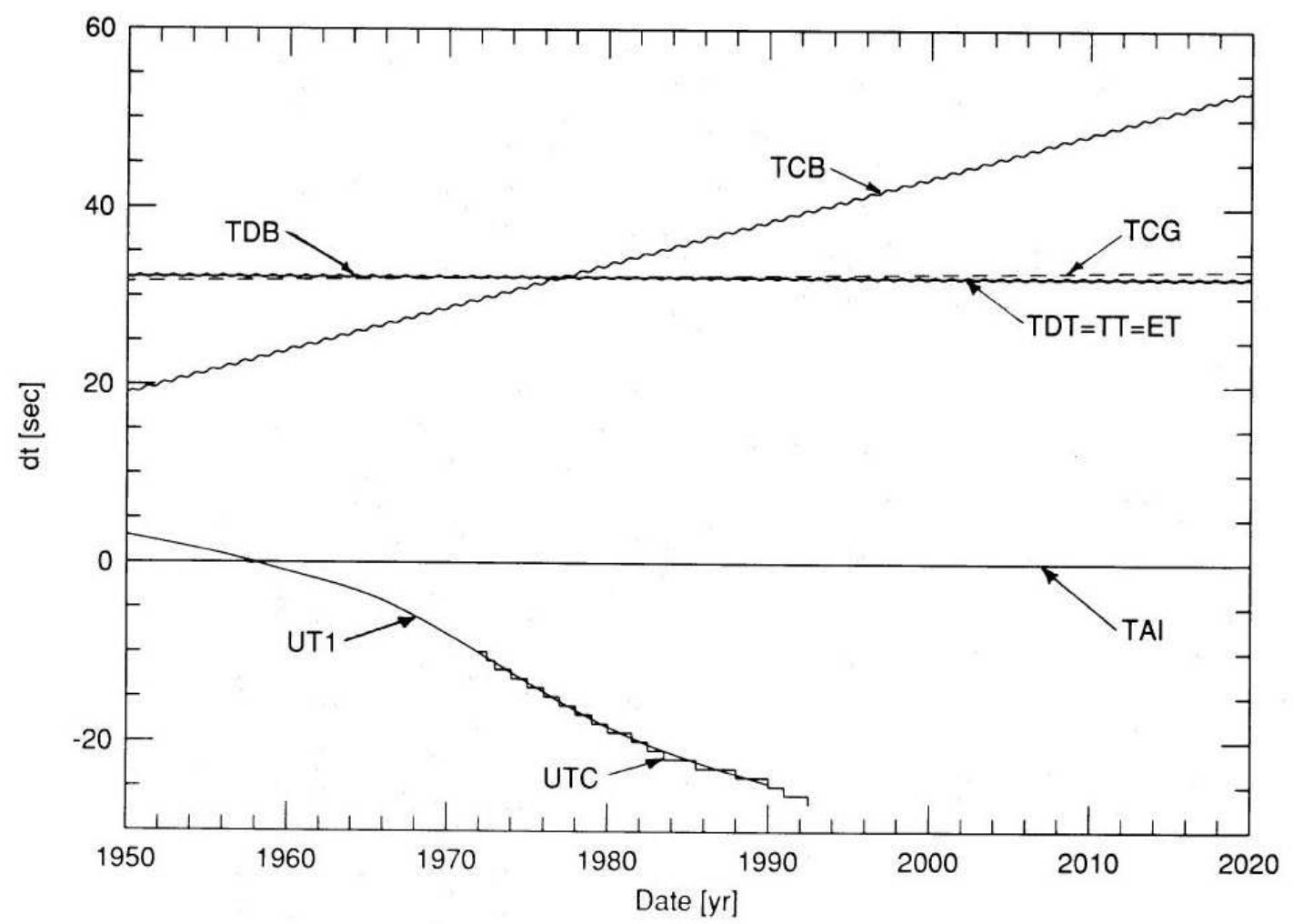

Figure 5.1: Comparison of different time standards. From Seidelmann \& Fukushima (1992). The periodic terms of TCB and TDB are magnified by 100 to make them visible.

the center of mass of the Solar system, the Solar System Barycenter (SSB) as reference point. The corresponding reference frame is the Barycentric Julian Date (BJD). In the following I will always use the barycentric frame.

- Time standard ${ }^{1}$ :

The time standard defines, among other things, the rate at which a clock is ticking. Figure 5.1 gives an overview of various time standards and their relation to each other. The most commonly used one (and most popular one in civil life) is Coordinated Universal Time, UTC. It was established in 1972 and is related to Universal Time UT1. However, UTC is discontinuous since it is not allowed to differ from UT1 by more than 0.9 seconds. If such a case approaches, a leap second is added to

$\begin{array}{ccccc}{ }^{1} \mathrm{An} \text { extensive collection of various time } & \text { standards is given in } \\ \text { http://www.ucolick.org/ }{ }^{\sim} \text { sla/leapsecs/timescales.html. }\end{array}$




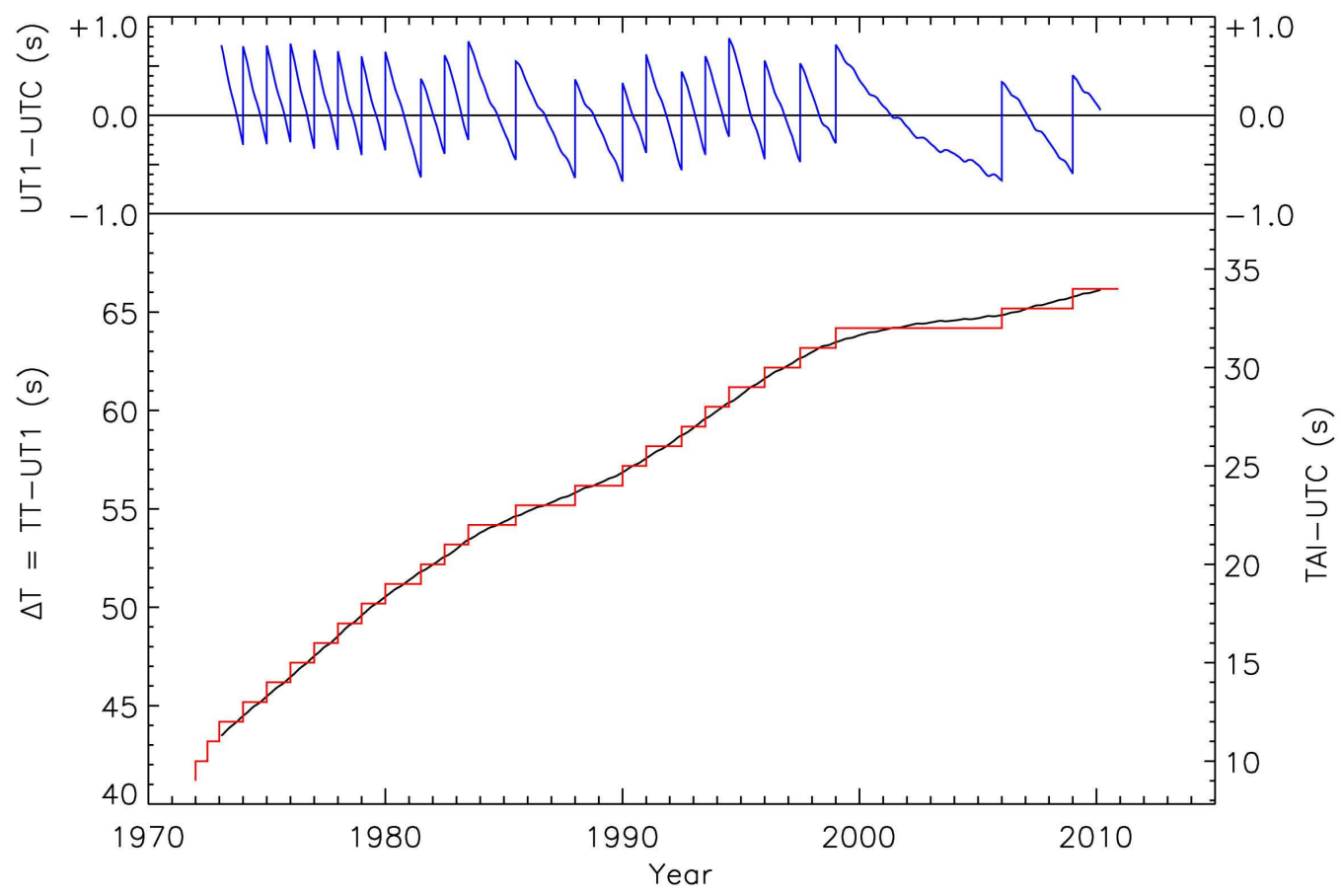

Figure 5.2: Leap seconds since UTC. Image credit: http://www.phys.uu.nl/〜vgent/deltat/deltat.htm.

UTC to account for the difference and stay within the \pm 0.9 s limit (see Fig. 5.2 for all leap seconds applied since 1972).

The International Atomic Time (TAI) is the basis for all other time standards. This time standard is a weighted average of the time kept by more than 200 atomic clocks. In 1972, when UTC was established, TAI was ahead of UTC by an initial difference of ten seconds. Since then, 24 leap seconds were applied to UTC, such that currently (as of the end of 2010), TAI is ahead of UTC by 34 seconds. Terrestrial Time (TT) runs at the same rate as TAI, but with a constant offset of 32.184 seconds (i.e. TT $=\mathrm{TAI}+32.184 \mathrm{~s}$, which has historical reasons coming from the difference of the TT precursor ET, Ephemeris Time, from TAI in 1972). The Barycentric Dynamical Time (TDB) also takes into account relativistic effects but this time standard is only necessary if accuracies better than milliseconds are required. In the following, we will therefore use TT as our time standard. 


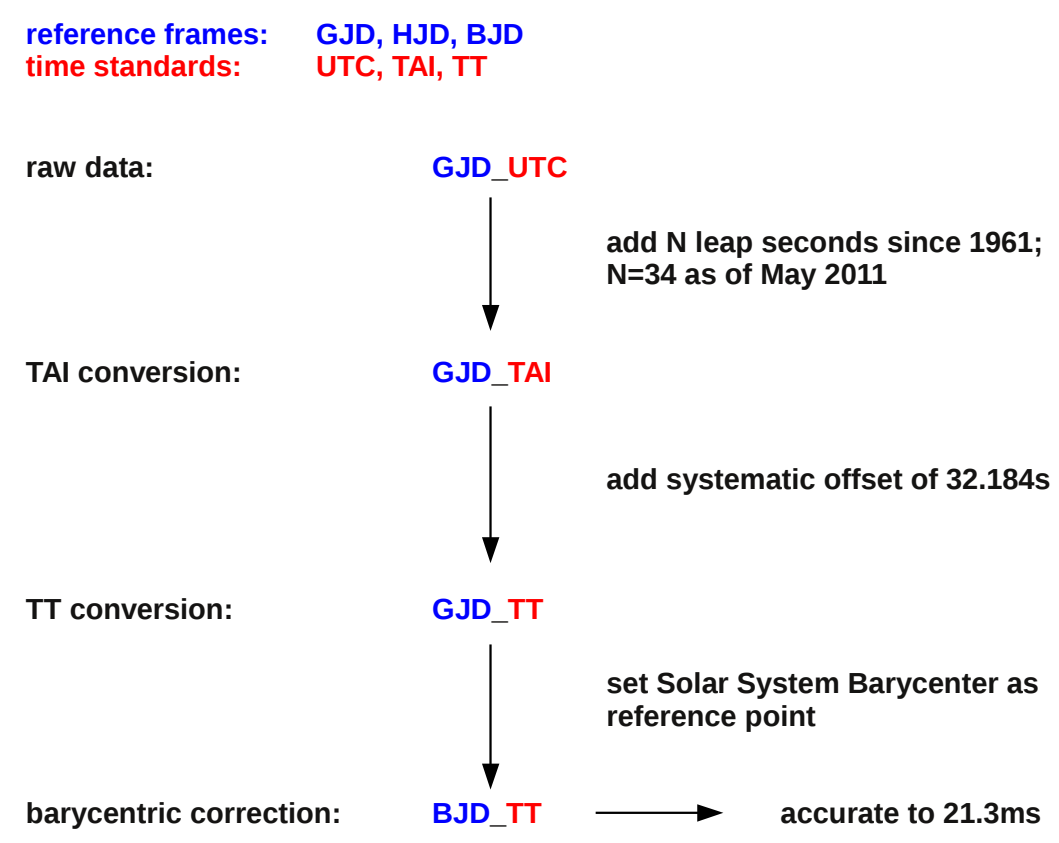

Figure 5.3: The ideal way to apply the time corrections. At the time of writing (May 2011) the number of leap seconds applied since 1961 is $\mathrm{N}=34$.

The Terrestrial Time as time standard combined with the BJD reference frame defines the time stamp BJD $\mathrm{TT}_{\mathrm{TT}}$. $\mathrm{BJD}_{\mathrm{TT}}$ is accurate to the ms level, which is precise enough for our purposes and we will use this time stamp as a basis for all our data. One also has to keep in mind that the FITS header time information recorded by the computer clocks at the observatories should, conservatively, not be trusted to be much better than $0.25 \mathrm{~s}$ anyway (depending e.g. on network connection, operating system, CCD soft- and hardware). Another important factor to keep in mind is to put the precise target coordinates into the barycentric correction. Putting in coordinates differing from the target's coordinates by 1 arcmin or 1 arcsec will induce a timing error of up to 0.28 seconds or up to 0.0048 seconds, respectively!

The raw data coming from our various observation sites are in UTC time standard and Geocentric Julian Date (GJD) reference frame (precisely there is a $21.3 \mathrm{~ms}$ error between the Earth's center and the Earth's surface as reference point, but since this is below our desired precision anyway, we 


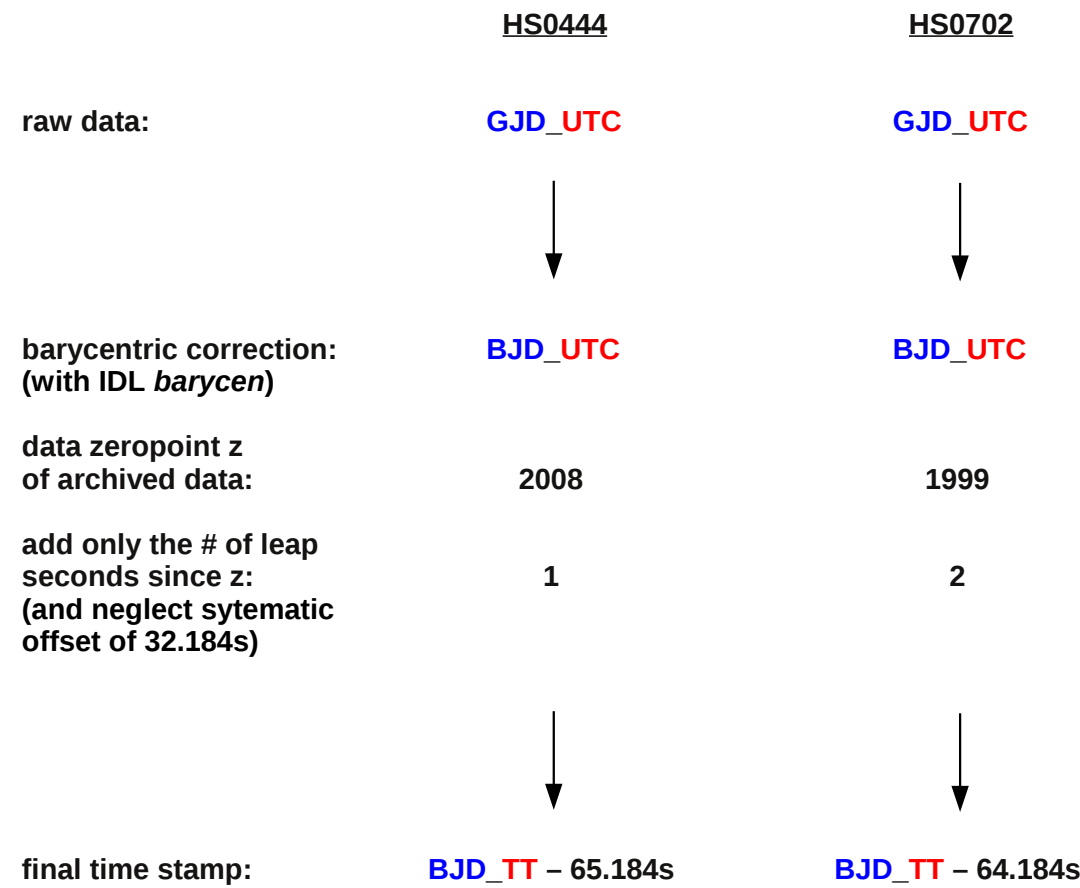

Figure 5.4: Time correction steps done for our data.

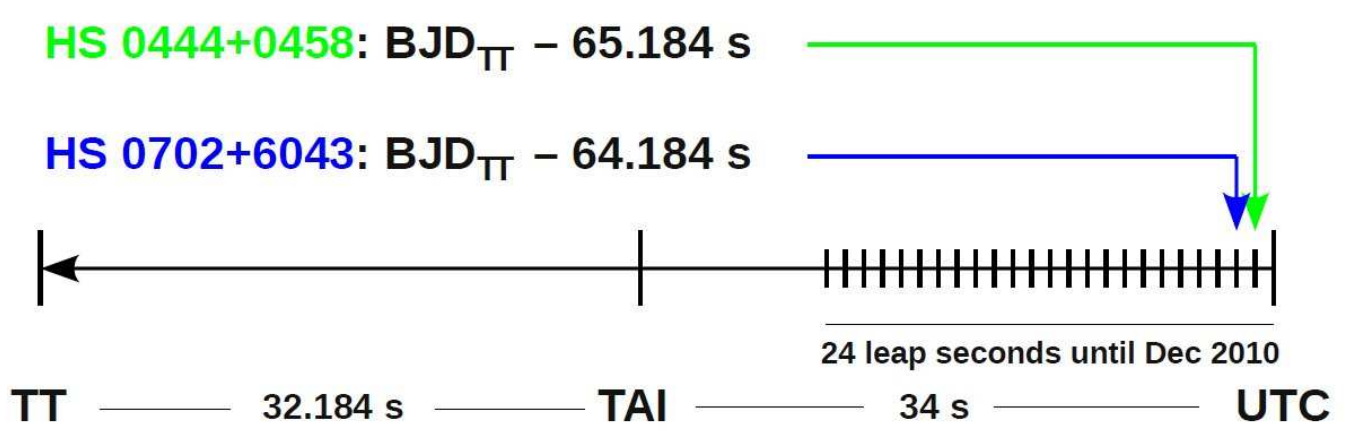

Figure 5.5: The final format of our data.

will still refer to the raw data taken by an arbitrarily located telescope on Earth's surface as GJD).

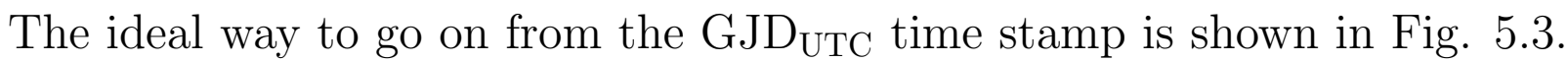
The final $\mathrm{BJD}_{\mathrm{TT}}$ is accurate to $21.3 \mathrm{~ms}$, which is precise enough for our purposes. However, we have done some different steps during our reduction (see Fig. 5.4) and I will outline our procedure of the treatment of the raw data in the following: 
We first convert the Geocentric Julian Dates of each single exposure to $\mathrm{RJD}_{\mathrm{UtC}}$ (RJD is the Reduced Julian Date, i.e. JD-2400000). Then we transfer this to $\mathrm{MJD}_{\mathrm{UTC}}$ with MJD being the Modified Julian Date, i.e. RJD-0.5. This step is only necessary because the routine we use to do the barycentric correction, the IDL $^{2}$ routine barycen $^{3}$ requires the input to be in Modified Julian Date format. This correction then yields BJD $\mathrm{UTC}_{\mathrm{UC}}$.

For historical data archive reasons, instead of adding the 34 seconds (from UTC to TAI) and the constant 32.184 seconds (from TAI to TT) to get the $\mathrm{BJD}_{\mathrm{TT}}$, we set our leap second zeropoint to the first individual data of our targets and only add the amount of leap seconds happened since then. The latest leap seconds happened on 31.12.2005 and 31.12.2008. For HS $0444+0458$ we set this zeropoint to 2008 since our first data are from August 2008 and we therefore add one second to all our BJD UTC $_{\text {data }}$ after 31.12.2008 and until the next leap second will be installed ${ }^{4}$. For our BJD UTC $_{\text {I }}$ data on HS 0702+6043 the zeropoint is in 1999 and therefore we add one second to all data after 31.12.2005 and two seconds (see Fig. 5.4) to all data after 31.12.2008 and until the next leap second will be installed. To ensure consistency with our current data sets, each future leap second has to be added accordingly to the scheme outlined above.

As shown in Figure 5.5, all our HS 0444+0458 data therefore have the time stamp BJD $\mathrm{TT}_{\mathrm{T}}-65.184 \mathrm{~s}$ and our HS 0702+6043 data $\mathrm{BJD}_{\mathrm{TT}}-64.184 \mathrm{~s}$ (i.e. we did not add the constant 32.184s offset and furthermore, instead of adding 34 leap seconds to all data, we only added one leap second to all HS 0444+0458 data after 31.12.2008 and one or two leap second(s) for HS 0702+6043 data after 31.12.2005 and 31.12.2008, respectively). I want to emphasize that this is only a constant offset and that the data are still closely related to the continuous time standard TT and not to the discontinuous UTC time standard. The offsets are only due to archival reasons and have neither discontinuities to the TT format nor physical impact on the data analysis.

\footnotetext{
${ }^{2}$ Interactive Data Language, http://www.ittvis.com

${ }^{3}$ http://astro.uni-tuebingen.de/software/idl/aitlib/astro/barycen.html

${ }^{4}$ Because of irregularities in the slow-down of Earth's rotation, one cannot foretell when it will be necessary to install the next leap second.
} 


\subsection{Time-series and frequency analysis}

To analyze our photometric time-series data, we use the program Period04 (Lenz \& Breger, 2004, 2005). It automatically determines sampling rates and Nyquist frequencies and performs least-squares fits of sine waves of the form

$$
f(t)=Z+\sum_{i} A_{i} \sin \left(2 \pi\left(\Omega_{i} t+\Phi_{i}\right)\right)
$$

For further information, I refer the reader to the given references.

During the pre-whitening process we subsequently subtract the dominant frequencies and redetermine (improve) the previously derived parameter simultaneously. This is done until no significant peaks are left in the periodogram. A common concept for multisite campaign data is given by Breger et al. (1993) who suggest that "a signal/noise amplitude ratio of $\approx 4.0 \mathrm{might}$ be a good criterion to distinguish between peaks due to pulsation and noise". Let $m$ be the relative intensity of a light curve, $A$ the amplitude, $\phi$ the phase and $\nu$ the frequency of a certain pulsation. We estimate the errors $\sigma$ of these quantities according to Montgomery \& O'Donoghue (1999) for the case of uncorrelated noise:

$$
\begin{aligned}
\sigma_{m} & =\text { standard deviation of light curve residuals } \\
\sigma_{A} & =\sqrt{\frac{2}{N}} \cdot \sigma_{m} \approx \text { mean of Fourier transform residuals }
\end{aligned}
$$

with $N$ being the number of data points

$$
\begin{aligned}
\sigma_{\phi} & =\sqrt{\frac{2}{N}} \frac{\sigma_{m}}{A}=\frac{\sigma_{A}}{A} \\
\sigma_{\nu} & =\sqrt{\frac{6}{N}} \frac{1}{\pi T} \frac{\sigma_{m}}{A}=\sqrt{3} \frac{1}{\pi T} \frac{\sigma_{A}}{A}=\sqrt{3} \frac{1}{\pi T} \sigma_{\phi}
\end{aligned}
$$

The error in the frequency may also be approximated by $\sigma_{\nu} \approx \frac{1}{T}$ with $T$ being the length of the observations (time baseline). This expression for $\sigma_{\nu}$ corresponds to the FWHM of the main peak in the periodogram. 


\section{$5.4 \quad$ Timing Method}

Since data from different telescope sites are of different quality, we applied a weighting scheme to the light curves. Point weights $w$ are assigned to each light curve intensity according to $w=\frac{1}{\sigma^{2}}$, with $\sigma$ being the standard deviation of the residual light curves, calculated on a subset of $n$ data points and centered on each time stamp. The residuals are obtained by subtracting all significant signals until only noise is left. This weighting scheme can be described as sort of a "moving standard deviation". $n$ is usually an integer corresponding to the number of data points within two or three pulsational cycles. Gaps are also taken into account during this procedure. For the weighting, a routine provided by R. Silvotti (priv. comm.. also see Silvotti et al., 2006) has been used. These weights were then used in the subsequent analysis with Period04. The treatment and especially the weighting of multi-site photometric data is also addressed in Handler (2003).

The $\mathrm{O}-\mathrm{C}$ diagram, as a particular application of a timing method, will be described in more detail in the following section. Also the various appearances and associated interpretations of $\mathrm{O}-\mathrm{C}$ diagrams are to be mentioned in a later section. Here I aim to focus on the basic concept of constructing an $\mathrm{O}-\mathrm{C}$ diagram.

The $\mathrm{O}-\mathrm{C}$ analysis is an approach to measure the phase variations of a periodic function. In our case, these periodic functions are the pulsations of the sdB star which lead to periodic intensity variations. Other applications of a timing method in exoplanet searches include the timing of Pulsar signals and the timing of eclipses in eclipsing binary systems. In all cases the periodic signal is used as a clock and one wants to examine the behavior of this clock as a function of time. To do so, it is reasonable to compare the calculated ("C"-part of the O-C diagram) mean ephemeris or light curve solution of the whole data set to actually observed ("O"-part of the O-C diagram) ephemeris or light curve solution to temporal subsets of the whole data set.

A detailed treatment of the division of our EXOTIME data into subsets will be given in the analysis chapter. For illustrative purposes let us assume we have one long data set, which is divided into three shorter subsets, as sketched in Figure 5.6. Of course, putting these three subsets together, will again provide the long data set. Let us term those shorter subsets as 


\section{full data set: calculated model C}

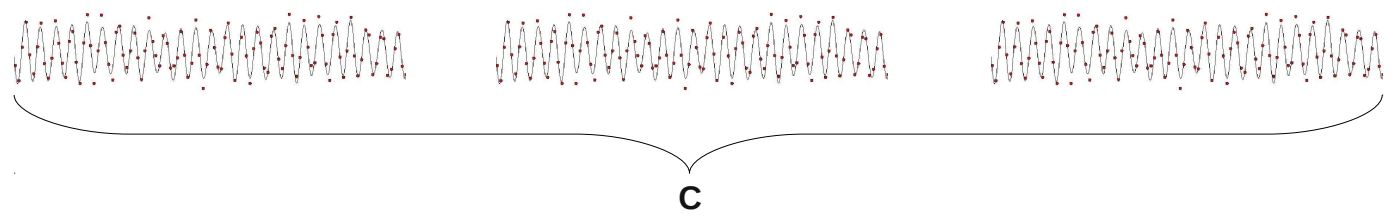

data subsets: observations $\mathbf{O}_{\mathrm{i}}$
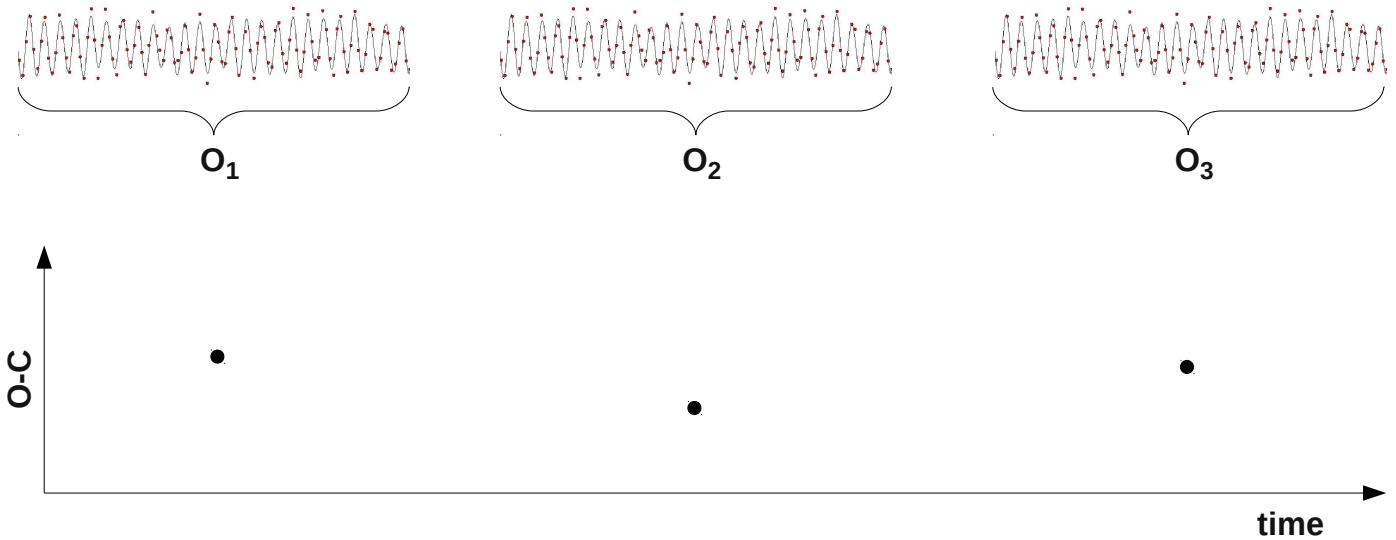

Figure 5.6: Principle of the $\mathrm{O}-\mathrm{C}$ method.

"seasonal" data and the complete long data set as "full" data, such that after the before mentioned corrections one now has a weighted full light curve and three weighted seasonal light curves. In a first step, a light curve solution is determined for the full light curve. As mentioned before, I will use the program Period04 (Lenz \& Breger, 2004, 2005) to do so. Since sdB stars are multimode pulsators, the solution will consist of a frequency, amplitude and phase for each pulsation considered. Let us only assume a single pulsation for the present basic concept. The frequency solution of this full light curve (the "C" part) is then kept fixed and each seasonal light curve is then fitted with that fixed frequency, while determining the amplitudes and phases of the seasonal light curves (the "O" parts). It might also be valuable to keep both the frequency and the amplitude of the full light curve fixed if desired ${ }^{5}$. For each seasonal light curve the phase difference to the full

\footnotetext{
${ }^{5}$ In our analysis, we will always keep the amplitudes free and therefore fit both the phases and the amplitudes in the subsets.
} 
light curve can now be determined. These phase differences are translated to time differences, which subsequently can be plotted as a function of time. Plotting these time differences as a function of time is one possible way to visualize an $\mathrm{O}-\mathrm{C}$ diagram.

In contrast to the examples in Figure 5.6, our real full data set (the "C" part) comprises several years of data and the subsets (the "O" parts) at least several nights of observations.

\subsection{The $\mathrm{O}-\mathrm{C}$ diagram}

In the previous section the general idea behind the timing method was outlined qualitatively. I will now derive the necessary formulas connected with an $\mathrm{O}-\mathrm{C}$ analysis and provide a mathematical description. Beginning with the case of a linearly changing period, I will continue with the description of the orbital light-time effect and the interpretation of certain features in an $\mathrm{O}-\mathrm{C}$ diagram with related physical situations. Afterwards I want to compare the sensitivity of the timing method to the sensitivities of various other exoplanetary detection methods. Finally I will go into more detail concerning the sensitivity in the special case of pulsating sdB stars.

A mathematical description of an $\mathrm{O}-\mathrm{C}$ diagram can be found in several places. In the next section I will follow Kepler et al. (1991), Kepler (1993), Kepler et al. (2005) and Mullally (2007). Additionally, nice general reviews of an O-C analysis may be found in Zhou (1999) or Sterken (2005).

\subsubsection{A linearly changing period}

Let there be a linearly changing period. The observed time $T_{E}$ of the $E^{\text {th }}$ cycle can be expressed in a Taylor series:

$$
T_{E}=T_{0}+\frac{d T}{d E}\left(E-E_{0}\right)+\frac{1}{2} \frac{d^{2} T}{d E^{2}}\left(E-E_{0}\right)^{2}+\ldots
$$

With $\frac{d T}{d E}=P$ being the period and $E_{0}=0$ one gets $\frac{d^{2} T}{d E^{2}}=\frac{d P}{d E}=\frac{d P}{d t} \frac{d t}{d E}=\dot{P} P$. Thus, identifying $T_{E}$ as the observed time of arrival, $O$, and neglecting terms 
higher than second order, one has

$$
O=T_{0}+P E+\frac{1}{2} P \dot{P} E^{2}
$$

and

$$
C=T_{0}^{\prime}+P^{\prime} E
$$

with $C$ being the expected arrival if a constant period $P^{\prime}$ is assumed. Defining $\Delta T=T_{0}-T_{0}^{\prime}$ and $\Delta P=P-P^{\prime}$, we can write

$$
O-C=\Delta T_{0}+\Delta P E+\frac{1}{2} P \dot{P} E^{2}
$$

Substituting the cycles $E$ with $\frac{t}{P}$ yields

$$
O-C=\Delta T_{0}+\frac{\Delta P}{P} t+\frac{1}{2} \frac{\dot{P}}{P} t^{2}
$$

Comparing the coefficients of a parabola of the form $c+b t+a t^{2}$ with the above expression gives us $c=\Delta T_{0}, b=\frac{\Delta P}{P}$ and finally $a=\frac{1}{2} \frac{\dot{P}}{P}$ or

$$
\dot{P}=2 a P
$$

$$
a \text { in } \frac{1}{d}, P \text { in } d .
$$

In an $\mathrm{O}-\mathrm{C}$ diagram, where the time difference is given in seconds and the time is given in days, the quadratic coefficient has the dimension $\left[\frac{s}{d^{2}}\right]$. Let us term this measured coefficient $\tilde{a}$. For a $\dot{P}$ with the dimension $\left[\frac{d}{d}\right]$ as above, one has to substitute $a$ with $\frac{\tilde{a}}{86400}$. This finally yields

$$
\dot{P}=2 \frac{\tilde{a}}{86400} P
$$

$$
\tilde{a} \text { in } \frac{s}{d^{2}}, P \text { in } d .
$$

The evolutionary timescale $T_{\text {evol }}$ can then be calculated with

$$
T_{\text {evol }}=P / \dot{P} \text {. }
$$

An alternative way of deriving equation 5.11 may be the following:

Let $t$ be the time, $P_{t}$ the true pulsation period being a function of time, 
i.e. $P_{t}=P(t)$, further $P_{c}$ the calculated period for the full data set (the "C"-part) and $E$ the cycle count, expressed by $E=\frac{t}{P_{c}}$.

We now define

$$
O \doteq P_{t} \cdot E=P_{t} \cdot \frac{t}{P_{c}}=\frac{P_{t}}{P_{c}} \cdot t
$$

and

$$
C \doteq P_{c} \cdot E=P_{c} \cdot \frac{t}{P_{c}}=t .
$$

A parabolic $\mathrm{O}-\mathrm{C}$ with coefficients $\mathrm{a}, \mathrm{b}$ and $\mathrm{c}$ can then be expressed as

$$
O-C=\left(\frac{P_{t}}{P_{c}}-1\right) \cdot t=a t^{2}+b t+c
$$

Differentiation of

$$
\frac{P_{t}}{P_{c}} \cdot t-t=a t^{2}+b t+c
$$

for $t$ yields (with usage of the product rule):

$$
\frac{1}{P_{c}} \cdot \frac{d P}{d t} \cdot t+\frac{P_{t}}{P_{c}}-1=2 a t+b
$$

Comparing the coefficients on the left and right side of

$$
\left(\frac{1}{P_{c}} \cdot \frac{d P}{d t}\right) \cdot t+\left(\frac{P_{t}}{P_{c}}-1\right)=2 a t+b
$$

shows:

$$
2 a=\frac{1}{P_{c}} \cdot \frac{d P}{d t} \text { and } b=\frac{P_{t}}{P_{c}}-1
$$

Therefore

$$
\frac{d P}{d t}=\dot{P}=2 a P_{c}
$$

If we now identify $P$ as the period of the full light curve solution (i.e. the "C"-part) and $a$ as the coefficient of the quadratic term in the parabolic fit to the $\mathrm{O}-\mathrm{C}$ diagram, the change of period is given by

$$
\dot{P}=2 a P \text {. }
$$




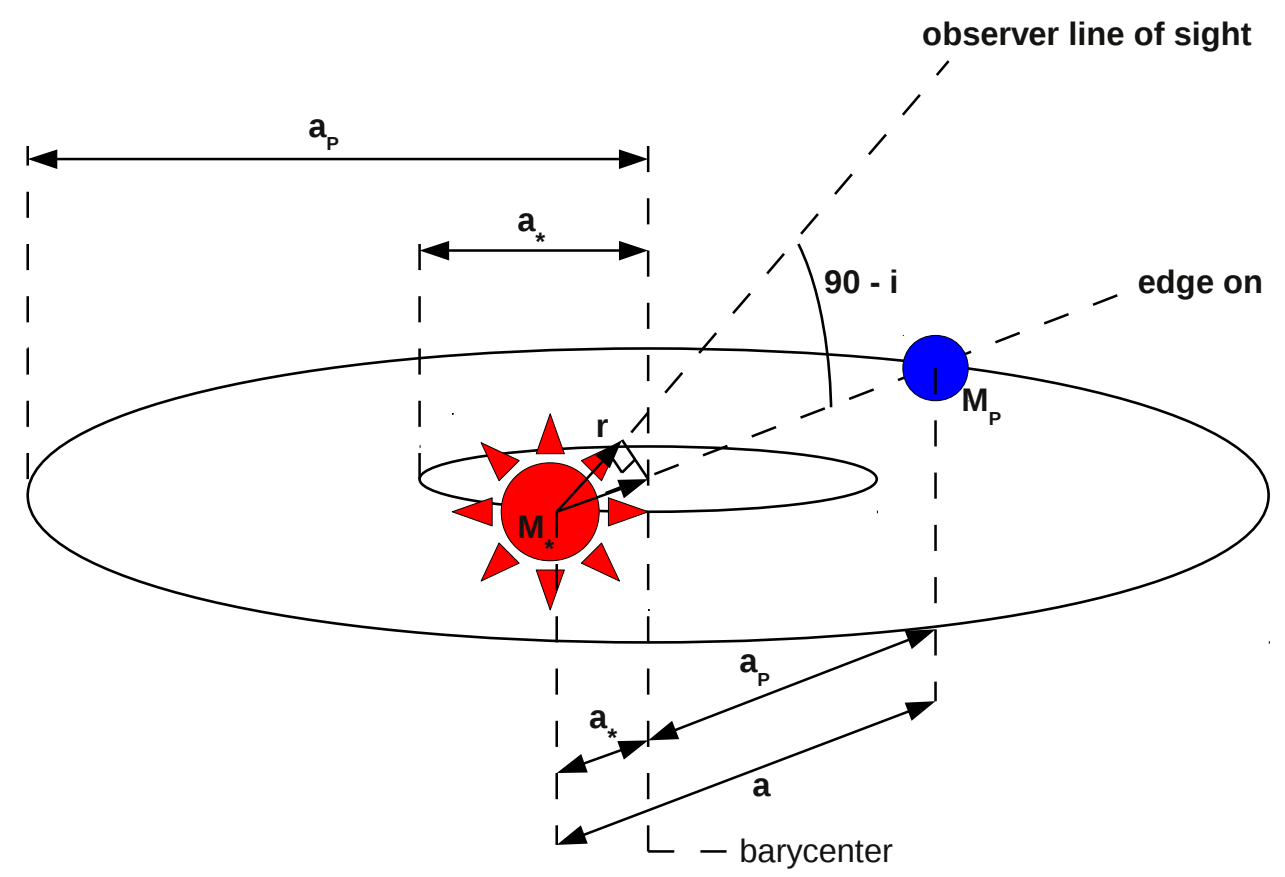

Figure 5.7: Sketch of orbital configuration. Here we assume circular orbits. The relations given are no longer valid for elliptical orbits.

\subsubsection{The orbital light-time effect}

I now want to provide a detailed description of the orbital light-time effect by calculating the $\mathrm{O}-\mathrm{C}$ (semi)-amplitude $\tau$ (half of the peak-to-peak amplitude):

Let us assume a two-body case in a circular orbit with a star of mass $M_{\star}$ and a planet of mass $M_{p}$. Let $r$ be the distance of the star from the common center of mass (barycenter), projected along the observer line of sight. Further, $i$ is the inclination ( $i=90$ degrees: edge on, $i=0$ degrees: face on), $a$ the orbital separation of the planet from the star and $a_{\star}$ and $a_{p}$ the distances of the star and the planet from the barycenter, as sketched in Figure 5.7. Looking at the geometry, $r$ can be derived via $\cos (90-i)=\frac{r}{a_{\star}}=\sin i$. 
With

$$
r=a_{\star} \cdot \sin i
$$

and

$$
a_{\star}=\frac{M_{p}}{M_{\star}+M_{p}} \cdot a
$$

one has

$$
r=\frac{M_{p}}{M_{\star}+M_{p}} \cdot a \sin i
$$

Expressing $r$ with the $\mathrm{O}-\mathrm{C}$ amplitude $\tau$ and the speed of light $c(r=\tau \cdot c)$ yields

$$
\tau=\frac{M_{p}}{M_{\star}+M_{p}} \cdot \frac{a \sin i}{c}
$$

and for $M_{p} \ll M_{\star}$ one gets

$$
\tau=\frac{M_{p}}{M_{\star}} \cdot \frac{a \sin i}{c}
$$

We now want to scale the planet's mass to Jupiter's mass, the stellar mass to the Solar mass and the separation to Astronomical Units.

$$
\tau=\frac{M_{p} / M_{J u p} \cdot 1.899 \cdot 10^{27} \mathrm{~kg}}{M_{\star} / M_{\odot} \cdot 1.9891 \cdot 10^{30} \mathrm{~kg}} \cdot \frac{a / A U \cdot 149597.870691 \cdot 10^{3} \mathrm{~km}}{299792.458 \frac{\mathrm{km}}{\mathrm{s}}} \cdot \sin i .
$$

Now the $\mathrm{O}-\mathrm{C}$ amplitude given in seconds can be expressed as

$$
\begin{aligned}
\tau=0.4764 \cdot \frac{M_{p}}{M_{\star}} \cdot a \cdot \sin i \\
\tau \text { in sec }, a \text { in } \mathrm{AU}, M_{\star} \text { in } M_{\odot}, M_{p} \text { in } M_{J u p} .
\end{aligned}
$$

For the following sensitivity estimations of the timing method, I will use the approximation

$$
\tau \approx 0.5 \cdot \frac{M_{p}}{M_{\star}} \cdot a \cdot \sin i
$$


The separation $a$ can be obtained via Kepler's 3rd law:

$$
\begin{aligned}
a^{3}=\left(M_{\star}+M_{p}\right) \cdot P_{\text {orb }}^{2} & \\
& a \text { in AU }, P_{\text {orb }} \text { in years }, M_{\star}, M_{p} \text { in } M_{\odot},
\end{aligned}
$$

with $P_{\text {orb }}$ being the orbital period.

Hence, by measuring the period of a sinusoidal signature in the $\mathrm{O}-\mathrm{C}$ (i.e. the orbital period $P_{\text {orb }}$ ) and estimating the stellar mass, we can calculate the orbital separation $a$. With this and with the measured amplitude of the sinusoidal signature in the $\mathrm{O}-\mathrm{C}$ (i.e. the amplitude $\tau$ ), one can finally derive the companion's minimum mass $M_{p} \cdot \sin i$ using equation 5.29 .

\subsection{Interpretation of $\mathrm{O}-\mathrm{C}$ diagrams}

The different cases which can appear in an $\mathrm{O}-\mathrm{C}$ diagram are shown schematically in Figures 5.8 and 5.9. If the variable star considered has a constant period, this will yield a linear signature in the $\mathrm{O}-\mathrm{C}$ diagram. If the estimated period is correct, the slope will be zero at $\mathrm{O}-\mathrm{C}=0$. A cycle count error results in a vertical shift while a positive slope is due to an estimated period shorter than the true period. These three cases are shown as green lines (the case of an estimated period too long results in a negative slope and is not shown in the Figure). If the period under consideration is changing linearly in time, the signature in the $\mathrm{O}-\mathrm{C}$ diagram is a parabola. The red line in Figure 5.8 shows the case of a period linearly increasing with time (parabola opened upward). A parabola opened downward (not shown here) indicates a linearly decreasing period. A sinusoidal signature (blue line in Figure 5.8) can be indicative of a reflex motion of the variable star due to the presence of an unseen companion. A review on these different cases can be found in Zhou (1999).

Other (physical) interpretations of linear or sinusoidal changes of the period include proper motions and beating effects of close frequencies. I will present a closer description of these during the analysis of our data.

If the construction of an $\mathrm{O}-\mathrm{C}$ diagram is done via phase differences, there is one important thing to be aware of. Usually the times of a certain event are considered. In our case this would be the times of maximum intensity caused 


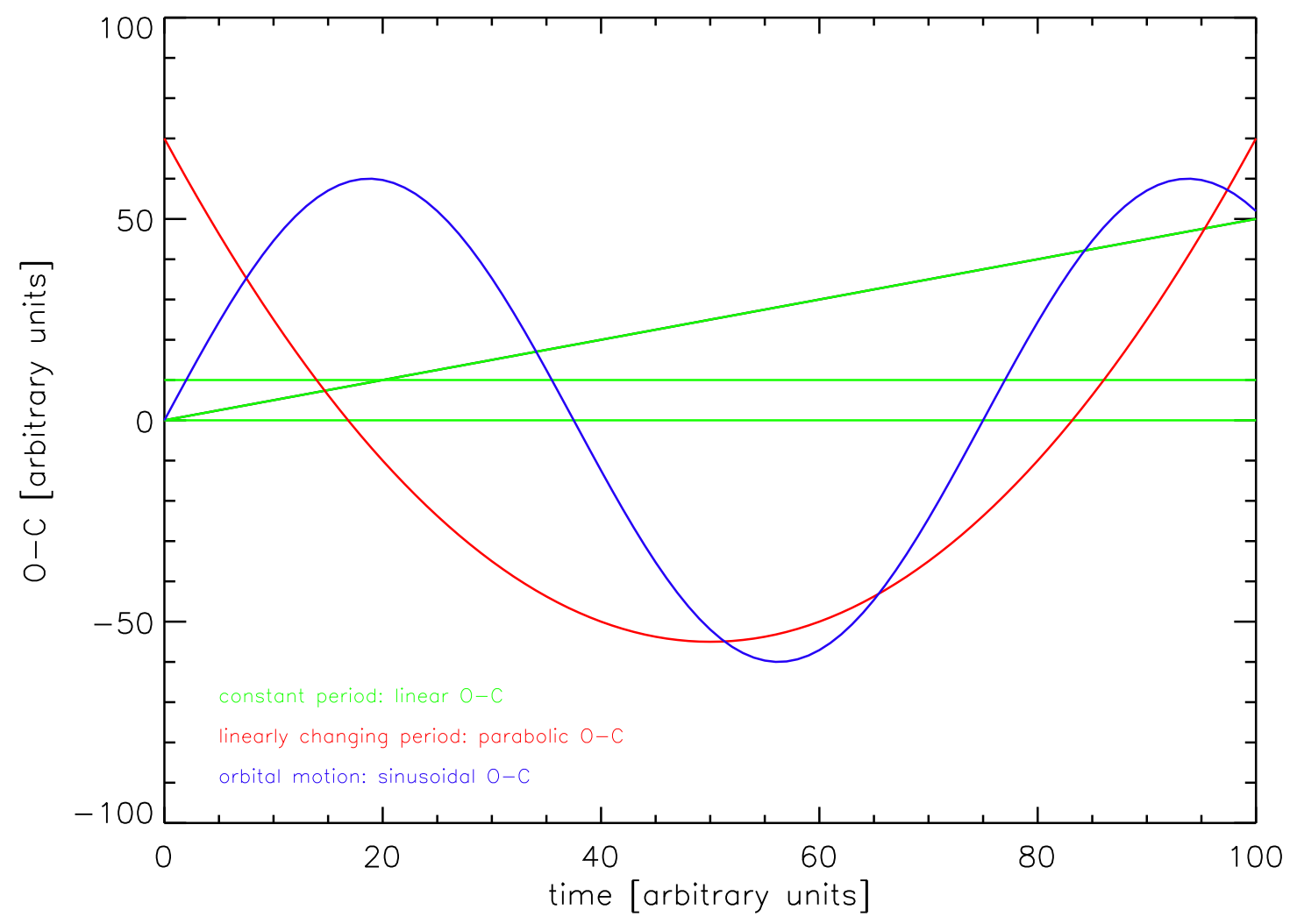

Figure 5.8: Different cases in an $\mathrm{O}-\mathrm{C}$ diagram. See text for further explanations.

by the pulsations. However, we first consider the phases. As can be seen in Figure 5.10, there is a factor of $(-1)$ between the $\mathrm{O}-\mathrm{C}$ diagrams derived via phase differences and time differences. This means that e.g. the downward parabola derived via phase differences in Figure 5.10 is an upward parabola if time differences are considered. Since we calculate phase differences but show time differences in all upcoming $\mathrm{O}-\mathrm{C}$ diagrams, we multiply all our calculated phase differences with the factor $(-1)$.

In a hypothetical case where a companion orbits a pulsating star, the resulting $\mathrm{O}-\mathrm{C}$ diagram would schematically look similar to Figure 5.11. The evolution of the star (if assumed to be linearly) would be revealed by the parabolic trend and the companion would reveal itself by a sinusoidal light time effect induced on the pulsating star. Both components together are shown as the blue line in Figure 5.11. This is to demonstrate the basic components. The overall shape will depend on the actual timescale of the stellar evolution and the orbital period and mass of the companion. 

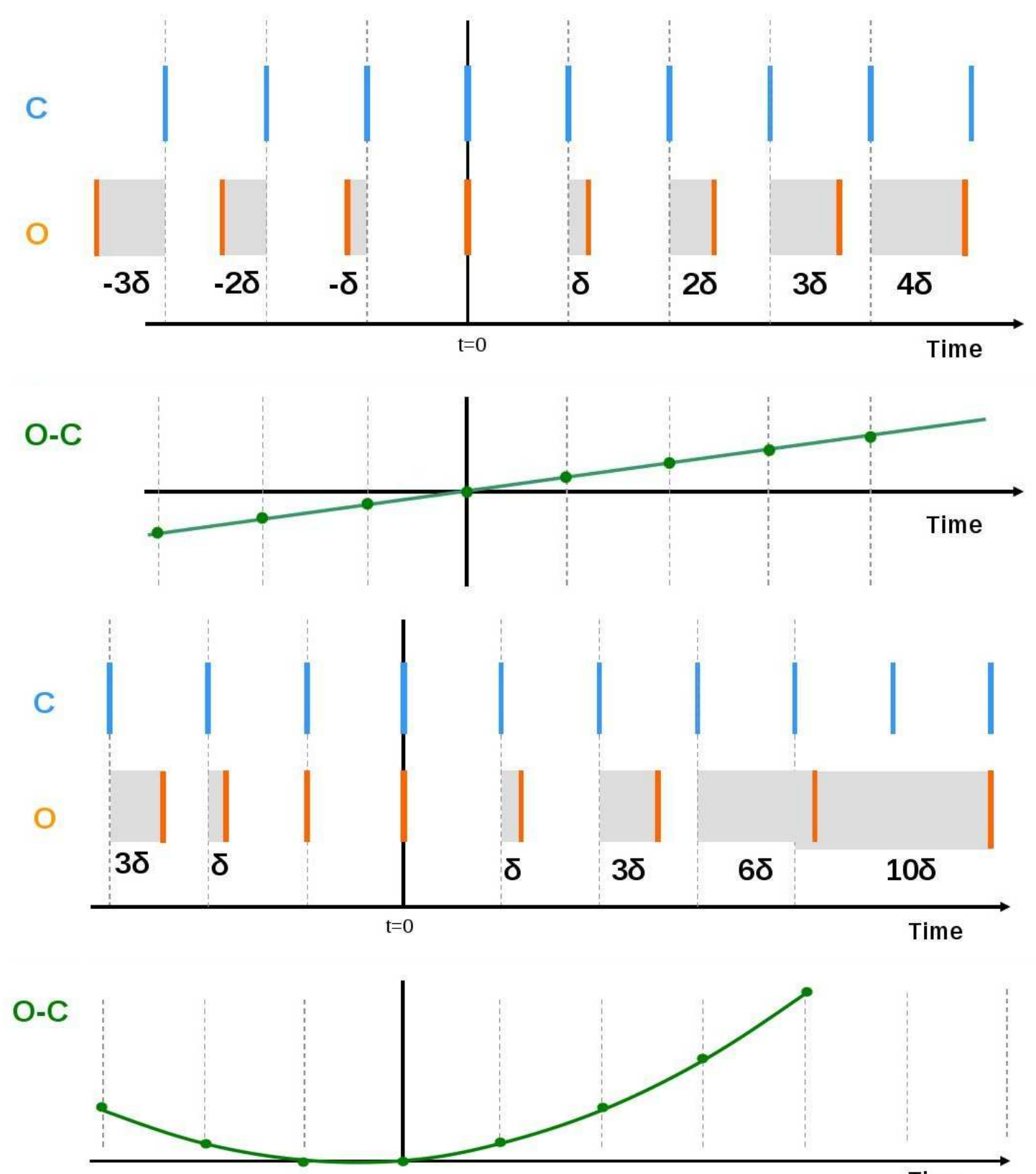

Figure 5.9: Illustrative construction of an $\mathrm{O}-\mathrm{C}$ diagram. Steps from the calculated model $\mathrm{C}$ and the observed events $\mathrm{O}$ to the $\mathrm{O}-\mathrm{C}$ diagram in the cases of a constant period (top) and a linearly changing period (bottom). From B. Barlow (priv.comm.).

\subsubsection{The case of HS $2201+2610$}

The discovery of a planetary companion around the pulsating sdB star HS 2201+2610 (Silvotti et al., 2007) finally triggered the idea of setting 


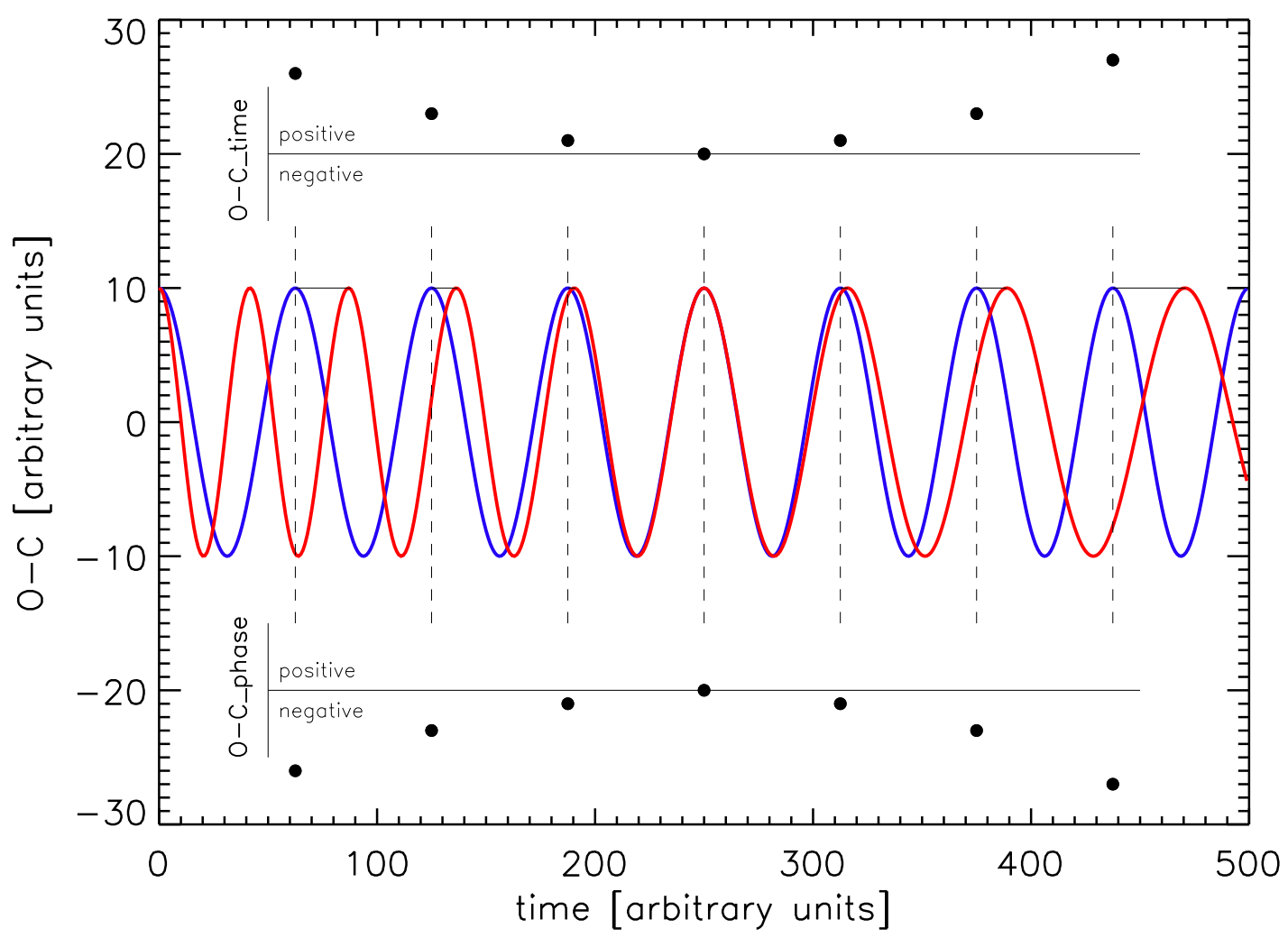

Figure 5.10: Occurrence of the factor -1 between the $\mathrm{O}-\mathrm{C}$ diagrams derived via times of an event (top) and phases of an event (bottom). In the case shown here, we assume a linearly increasing period. Blue: calculated $(\mathrm{C})$, red: observed $(\mathrm{O})$.

up the EXOTIME program. I will now provide a closer look on this system. Figure 5.12 shows the $\mathrm{O}-\mathrm{C}$ diagrams constructed independently for two frequencies. The top panels include both components, a parabolic and a sinusoidal one, while the bottom panels show the sinusoidal components alone. The diagrams are constructed on the basis of 418 hours of timeseries photometry taken in 167 observing night during the time span of the years 2000 to 2006 . For these multi-site observations, 18 different telescopes in the 1-3 meter range were used.

The $\dot{P}$ values and evolutionary timescales according to f1 and f2 are

- $\dot{P}_{1}=1.46 \pm 0.07 \cdot 10^{-12}, T_{e v o l, 1}=7.6 \mathrm{Myr}$

- $\dot{P}_{2}=2.05 \pm 0.26 \cdot 10^{-12}, T_{\text {evol }, 2}=5.5$ Myr, respectively. 


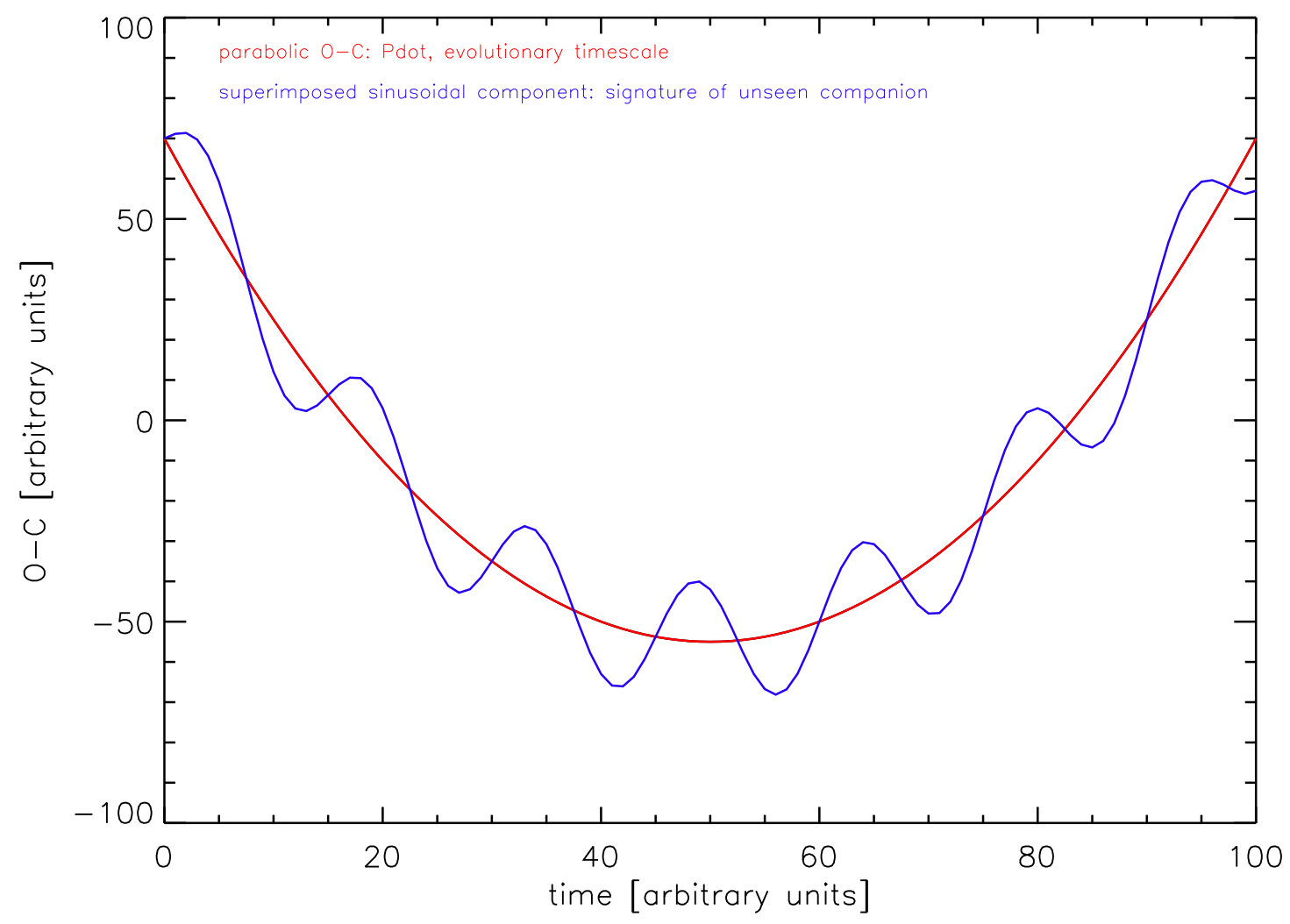

Figure 5.11: Orbital signature in an $\mathrm{O}-\mathrm{C}$ diagram.

Since both frequencies independently yield a sinusoidal signature with the same orbital period, orbital phase and $\mathrm{O}-\mathrm{C}$ amplitude, it is plausible to connect these signatures with the presence of a planetary companion. The long timescale of the sinusoidal variation of the order of years is much longer than the dynamical timescale of a sdB star which is roughly of the order of some minutes. An interpretation of the sinusoidal $\mathrm{O}-\mathrm{C}$ variation with a pulsational or evolutionary model is therefore implausible. Random period variations are also excluded by Silvotti et al. (2007) due to the large number of pulsation cycles in each data subset which would cancel these out.

Under the assumption of circular orbits ${ }^{6}$, the $\mathrm{O}-\mathrm{C}$ diagrams predict a planetary companion with a minimum mass of $3.2 \pm 0.7$ Jupiter masses orbiting HS $2201+2610$ at a distance of $1.7 \pm 0.1$ AU with a period of $3.20 \pm 0.12$ years. There are some scenarios proposed to explain the current configuration of

\footnotetext{
${ }^{6}$ This is justified because the $\mathrm{O}-\mathrm{C}$ diagrams do not show a significant deviation from a sinusoidal shape.
} 

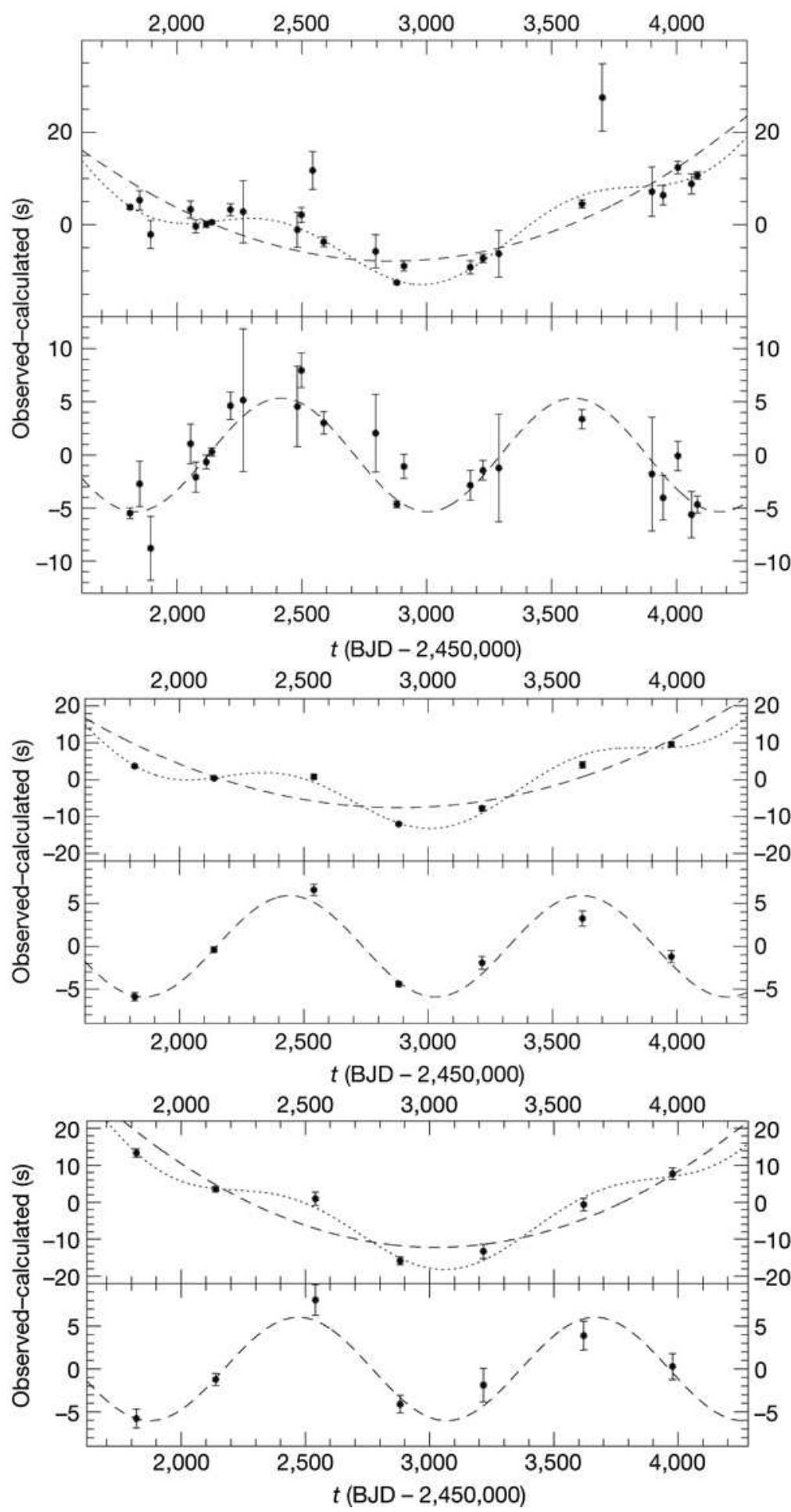

Figure 5.12: O-C diagrams for HS 2201+2610: f1, f1 binned, f2 binned (from top to bottom). The binning reduces the noise, but also the temporal resolution. Plots are from Silvotti et al. (2007). 
the system as well as its previous evolution. In a scenario where tidal effects are neglected, the distance of the planet during the main sequence phase of the sdB progenitor was closer to its host star and expanded to its present separation owing to the mass loss of the star which decreased the binding energy in the system. If tidal forces played a role ${ }^{7}$ the planet would have transferred angular momentum to the stellar envelope and spiraled towards it. Since a red giant radius much larger than $1 \mathrm{AU}$ is hard to explain, the present location of the planet at $1.7 \mathrm{AU}$ is only possible if we imagine a phase where the planet was inside the envelope (i.e. at a separation $\lesssim 1$ AU) and then, due to a severe mass loss (maybe even related to the helium flash) was pushed outwards to its present location. If the planet is believed to never have entered the envelope, it could still be a tracer for a further close-in planet which triggered the mass loss but was destroyed during that process.

Theories of the interplay and balance between inward drag due to tidal forces and outward push due to mass loss are unfortunately not very well understood yet.

Another possible scenario for the HS 2201+2610 system includes the formation of the sdB as a result of a merger event formation channel (as sketched in the introduction). In this case the planet would have formed out of the material of the disrupted component in a protoplanetary-like disk. In contrast to the scenarios outlined before, this scenario would define a second generation planet as in the case of the pulsar planets.

\subsection{Sensitivity of the Timing Method}

As of 15 April 2011 there were 543 exoplanets confirmed ${ }^{8}$. Among these, 12 planets are found with the timing method, 21 by imaging, 12 with microlensing and 498 by the radial velocity or astrometry method. The latter number also contains 127 transiting planets, which were found with the transit method. The transiting planets are included in the radial velocity group because any transiting planet is only a planet candidate until it is confirmed by radial velocity measurements. The different success rates of the various

${ }^{7} \mathrm{I}$ assume this to be the case and will expand on this in the discussion.

${ }^{8}$ According to http://exoplanet.eu 


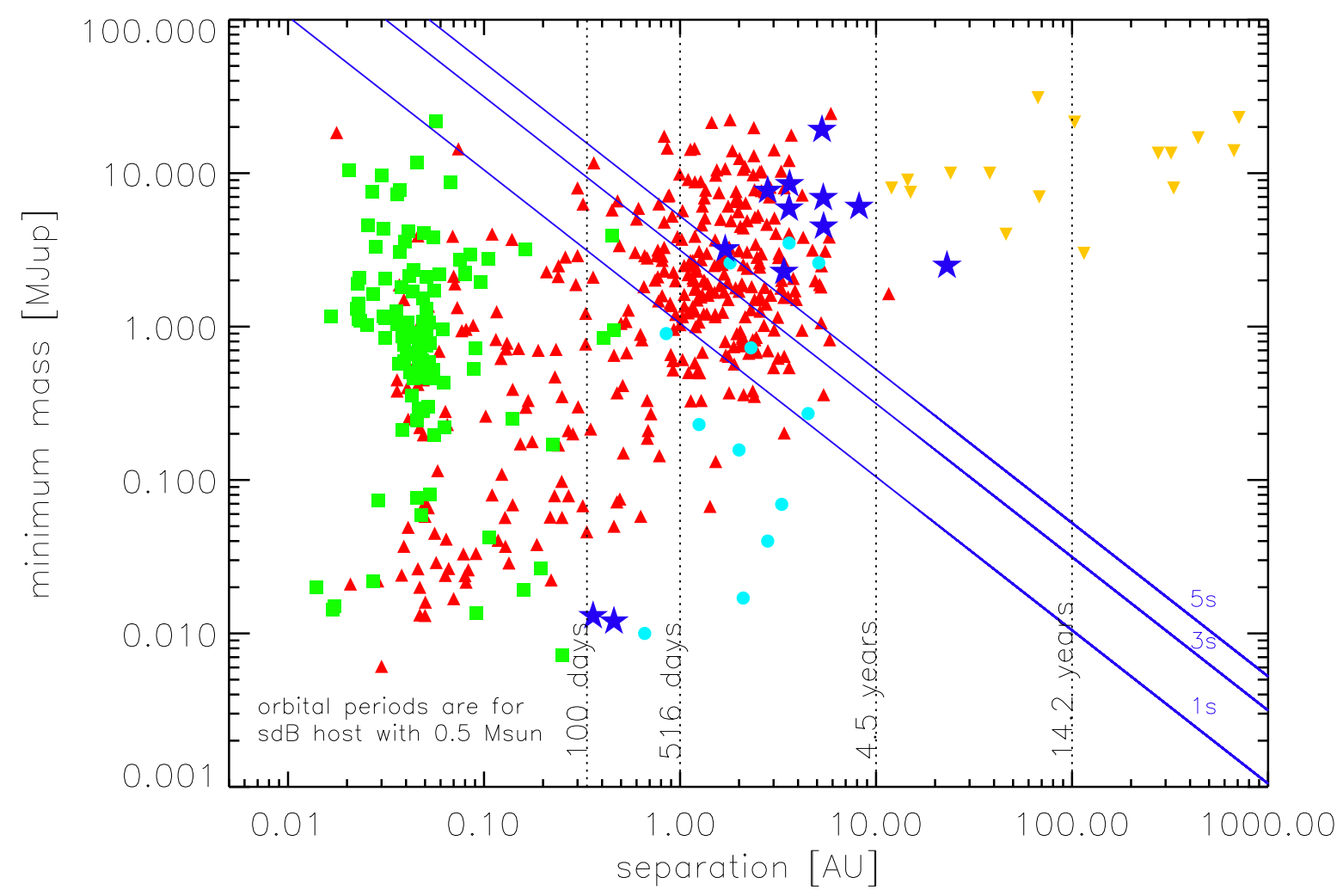

Figure 5.13: Comparison of the timing method to other exoplanet detection methods. The symbols are as in Figure 1.11 and the positions are taken from http://exoplanet.eu/. The solid lines mark the sensitivity of the timing method applied to pulsating sdB stars of half a solar mass for three different amplitude limits. The vertical dotted lines connect the orbital period to the corresponding separations.

detection methods are based on the physics of the methods themselves and also on the observational requirements.

The present section intends to compare the sensitivities of the various detection methods, with a focus given on some characteristics of the timing method. An application to the Solar System will be given as well as an estimation concerning close-in stellar companions.

Re-arranging equation 5.30 and solving for the minimum planetary mass yields a simple relation with the $\mathrm{O}-\mathrm{C}$ amplitude $\tau$ and the orbital separation $a$, if one assumes a sdB mass of roughly $0.5 M_{\odot}{ }^{9}: M_{p} \cdot \sin i \approx \tau \cdot \frac{1}{a}$. The

\footnotetext{
${ }^{9}$ The canonical sdB mass is often assumed to be $0.48 M_{\odot}$. The exact equation must therefore be derived
} 


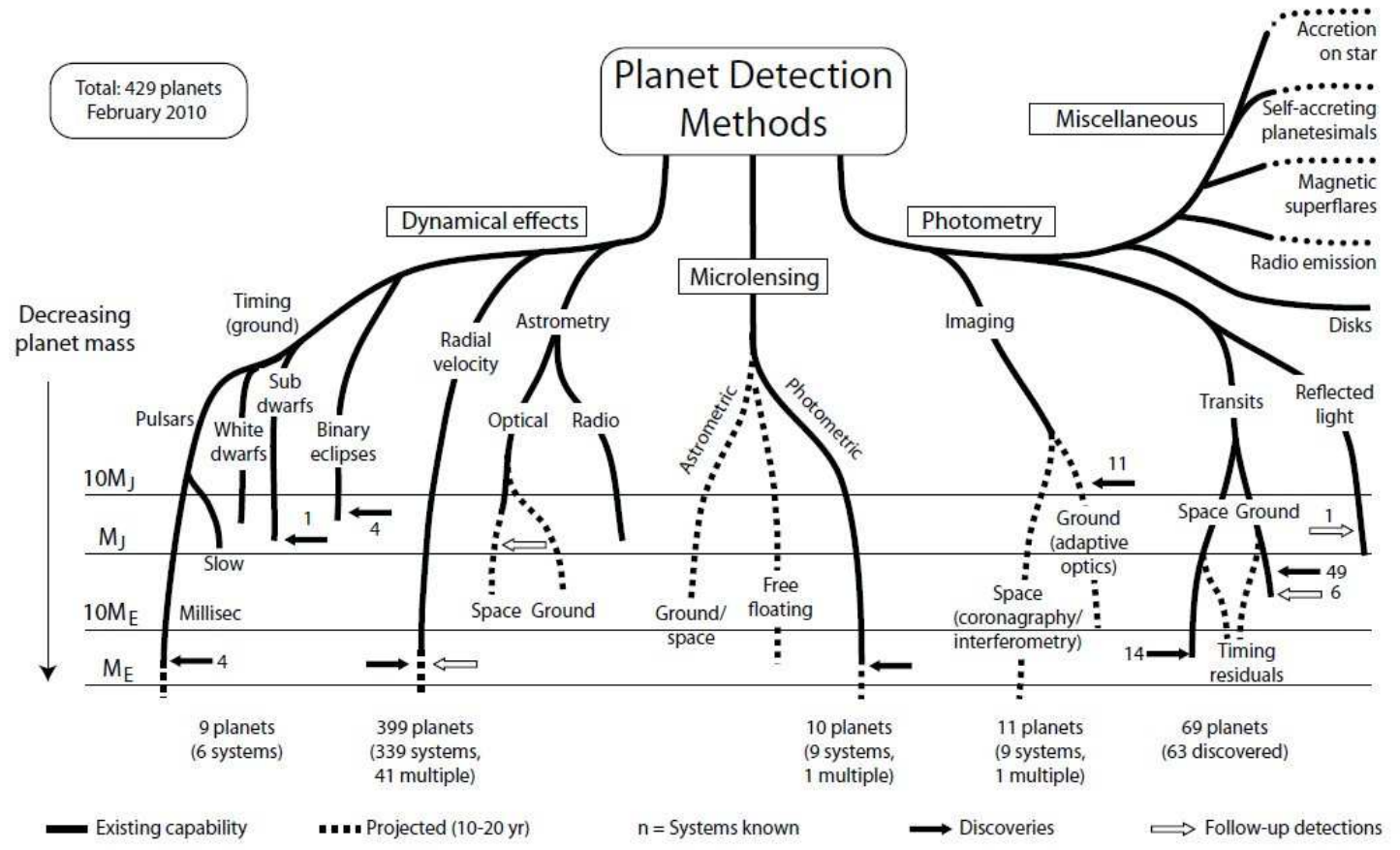

Figure 5.14: The "Perryman Tree" showing the capabilities of various detection methods. The figure is taken from Schuh (2010) and is an adopted version of Figure 1 in Perryman (2000), updated to include recent detections up to February 2010 (courtesy of M. A. C. Perryman).

$\mathrm{O}-\mathrm{C}$ amplitude $\tau$ is the quantity measured in the $\mathrm{O}-\mathrm{C}$ diagram, such that assuming different given amplitude sensitivities, one can plot the planetary mass versus the orbital separation. This has been done for different timing sensitivities in Figure 5.13 and can be compared to the parameter space accessible with other detection methods. As can be seen, the radial velocity method is most sensitive to close-in, massive objects. The same holds for the transit method, at least what concerns the orbital separation: the closer a planet is, the higher is the probability to actually see it transiting. The transit probability also increases for larger radii of the planets. The transit method is capable to detect even very low mass planets, which goes down to Earth mass planets in the case of space based observations like Corot or Kepler. However, a drawback of this method is the bias towards high inclinations close to 90 degree (edge on). It is obvious that the observer's line

from equation 5.29] $\tau=0.4764 \cdot \frac{M_{p}}{0.48 M_{\odot}} \cdot a \cdot \sin i$ and therefore $M_{p} \cdot \sin i=\frac{0.48}{0.4764} \cdot \tau \cdot \frac{1}{a}$. For our sensitivity estimations, however, we use the approximation given in the text and emphasize that this is valid since the differences are very little. In the data analysis of course the exact formula is used. 


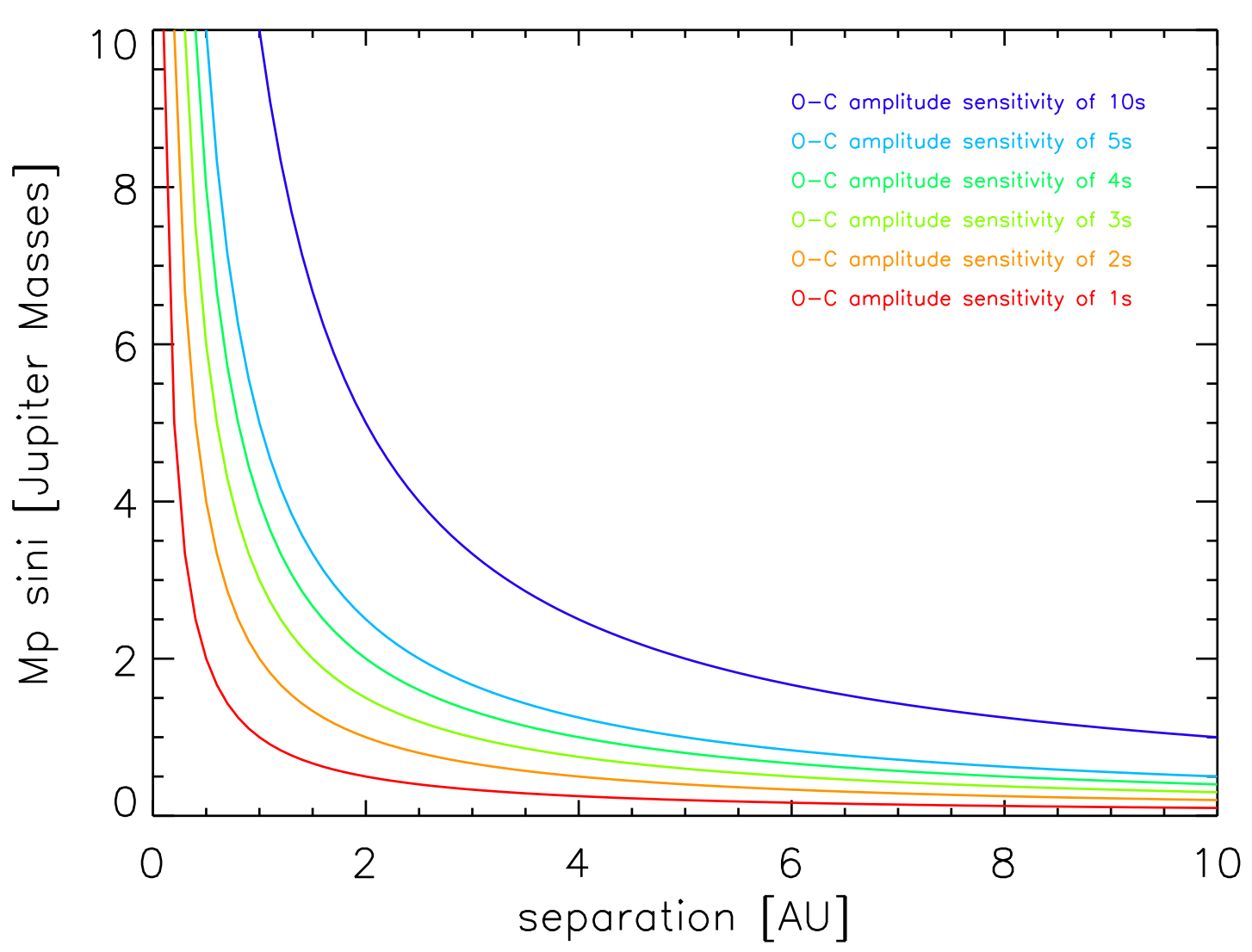

Figure 5.15: Sensitivity of the timing method applied to pulsating sdB stars for different $\mathrm{O}-\mathrm{C}$ amplitudes between 10s (uppermost line) and 1s (lowermost line).

of sight must coincide with the planet's orbital plane to detect the transit. This bias towards 90 degree on the other hand has the advantage that the inclination is known and that follow-up RV measurements, given that these reach the necessary sensitivity, could therefore determine the true masses of the planets. Hence, information found from the transit method (radius and inclination) and the RV method (radial velocity, binary mass function) can be combined to derive the true mass and ultimately the medium density of the planet, which is important to be able to separate gaseous from rocky planets.

Microlensing has the advantage to be able to detect very low masses but there is also the disadvantage that due to the physical configuration of this method, any detection cannot be re-observed since it is a "one time event". Also follow-up observations of a low mass microlensing event are in most 


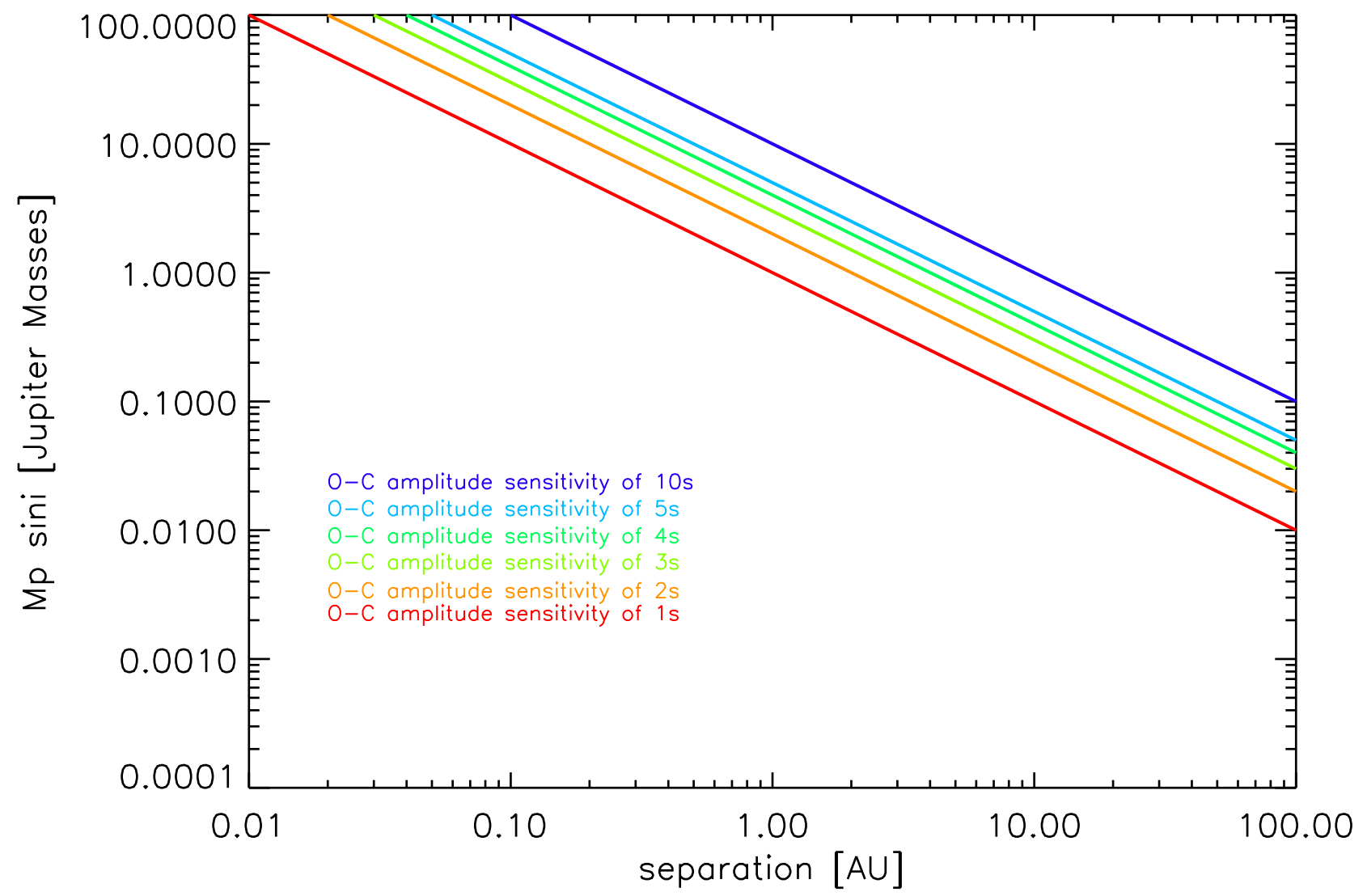

Figure 5.16: Same as Figure 5.15 but on logarithmic scales.

cases unsuccessful since the other methods do not reach the required sensitivity.

Direct imaging of planets is most sensitive to large orbital separations, since the host star and the planets usually have very high contrasts and the stellar light has to be "blocked" similar to a solar coronograph.

Figure 5.14 shows the famous "Perryman Tree", which also compares the capabilities of various detection methods.

The sensitivity limits of the timing method in the special case of sdB host stars within the EXOTIME program are given for different cases. We assume different $\mathrm{O}-\mathrm{C}$ amplitude sensitivities between $10 \mathrm{~s}$ as a pessimistic upper limit and $1 \mathrm{~s}$ as a lower limit (see Figures 5.15 and 5.16). The justification of these assumptions mainly depends on the total time base of 


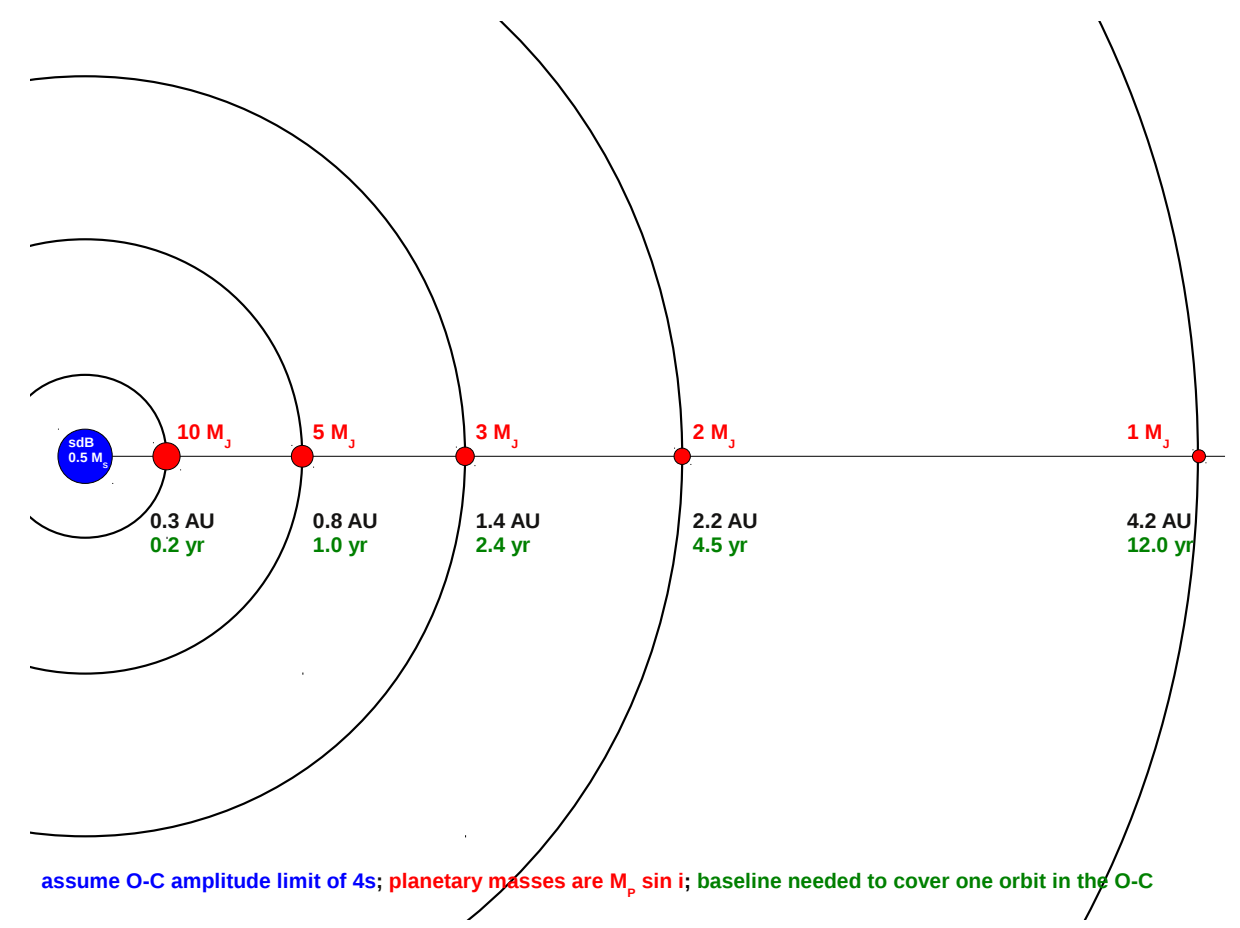

Figure 5.17: Detection limits for a hypothetical O-C amplitude of 4s.

observational data being available and the length of the seasonal subsets. As can be seen, the timing method is more sensitive to larger separations. This seems to make it complementary to the radial velocity method, but it is the different types of target stars which makes this argument inane. Our sdB stars are difficult targets for such precise RV measurements as would be needed to detect planetary companions around them: the low brightness and high surface gravities in combination with a lack of a large number of sharp spectral lines to conduct radial velocity measurements represent the most difficult issues. However, O'Toole et al. (2011) and Classen et al. (2011) investigate the applicability of the RV method to bright sdB stars in order to search for substellar companions.

Figure 5.17 shows the capability of the timing method if we assume a sensitivity of $4 \mathrm{~s}$. The larger the separation becomes, the more time is needed to cover a full cycle in the $\mathrm{O}-\mathrm{C}$ diagram.

I will now discuss the question if we could detect close-in stellar companions around a $0.5 M_{\odot}$ sdB pulsator with the timing method via ground-based observations. Let us assume an $\mathrm{O}-\mathrm{C}$ amplitude sensitivity of $5 \mathrm{~s}$. As can be seen in Fig. 5.18, the sdB wobble induced by a stellar companion would 


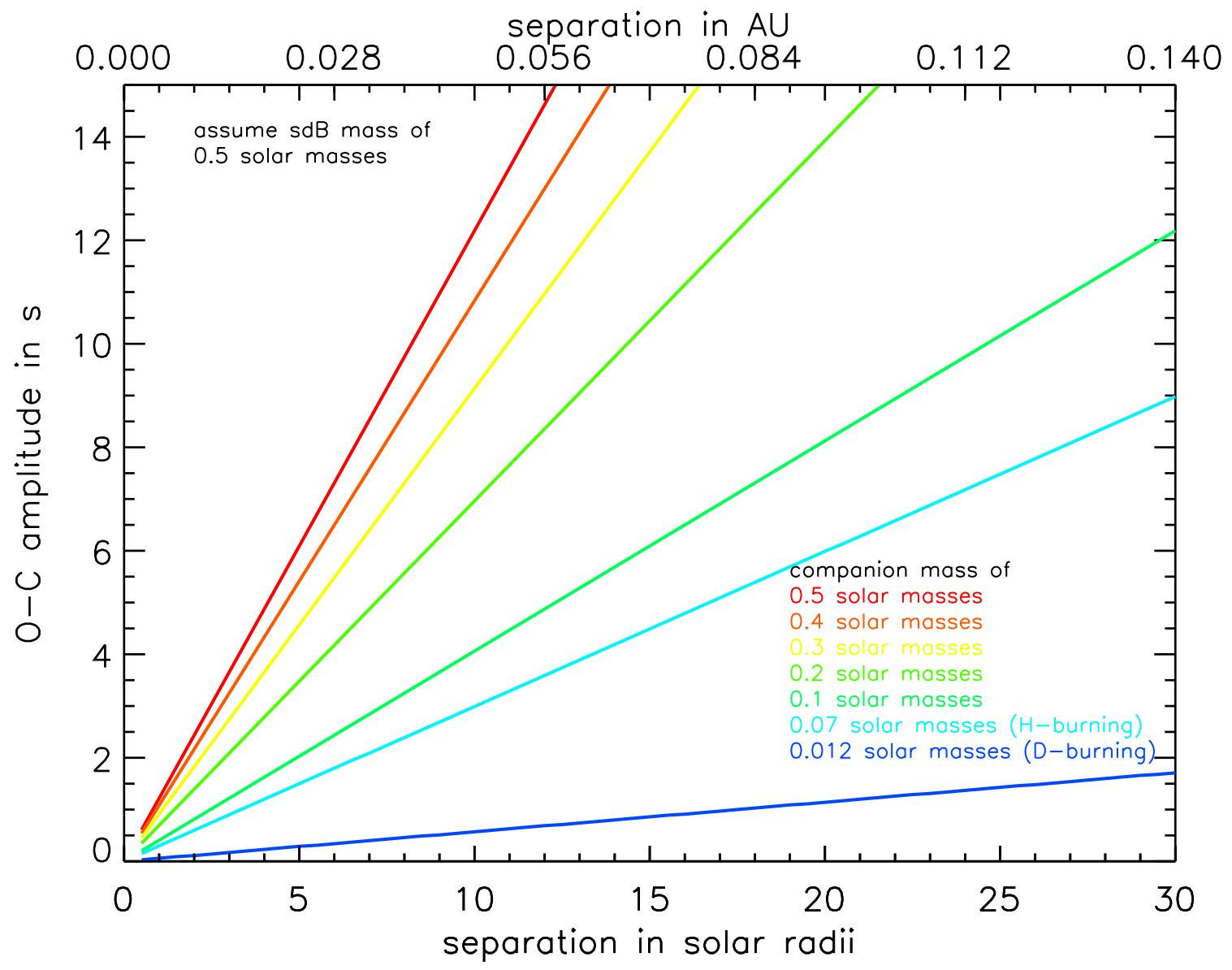

Figure 5.18: $\mathrm{O}-\mathrm{C}$ amplitudes of hypothetical close stellar companions.

be detectable if the orbital separation $a$ is $>4 R_{\odot}$ for a $0.5 M_{\odot}$ companion ( $>7 R_{\odot}$ for a $0.2 M_{\odot}$ companion and $>12 R_{\odot}$ for a $0.1 M_{\odot}$ companion). But at such close separations the orbital period is only $P_{\text {orb }}<5$ days (see Fig. 5.19). This baseline is too short to construct a meaningful $\mathrm{O}-\mathrm{C}$ diagram by means of observations from the ground.

For ground based data it is reasonable to assume that a sufficient frequency resolution for the pulsations in sdB stars can be achieved after three consecutive nights of observations. This subset will then yield one single point in the $\mathrm{O}-\mathrm{C}$ diagram. Let us now assume to start the next three day observational block right afterwards. This strategy would then produce an $\mathrm{O}-\mathrm{C}$ point every three days. If one wants to have at least six $\mathrm{O}-\mathrm{C}$ points to sample a full orbital period, this translates to a total baseline of at least 18 days. Applying this observational strategy sketched above (18 days of con- 


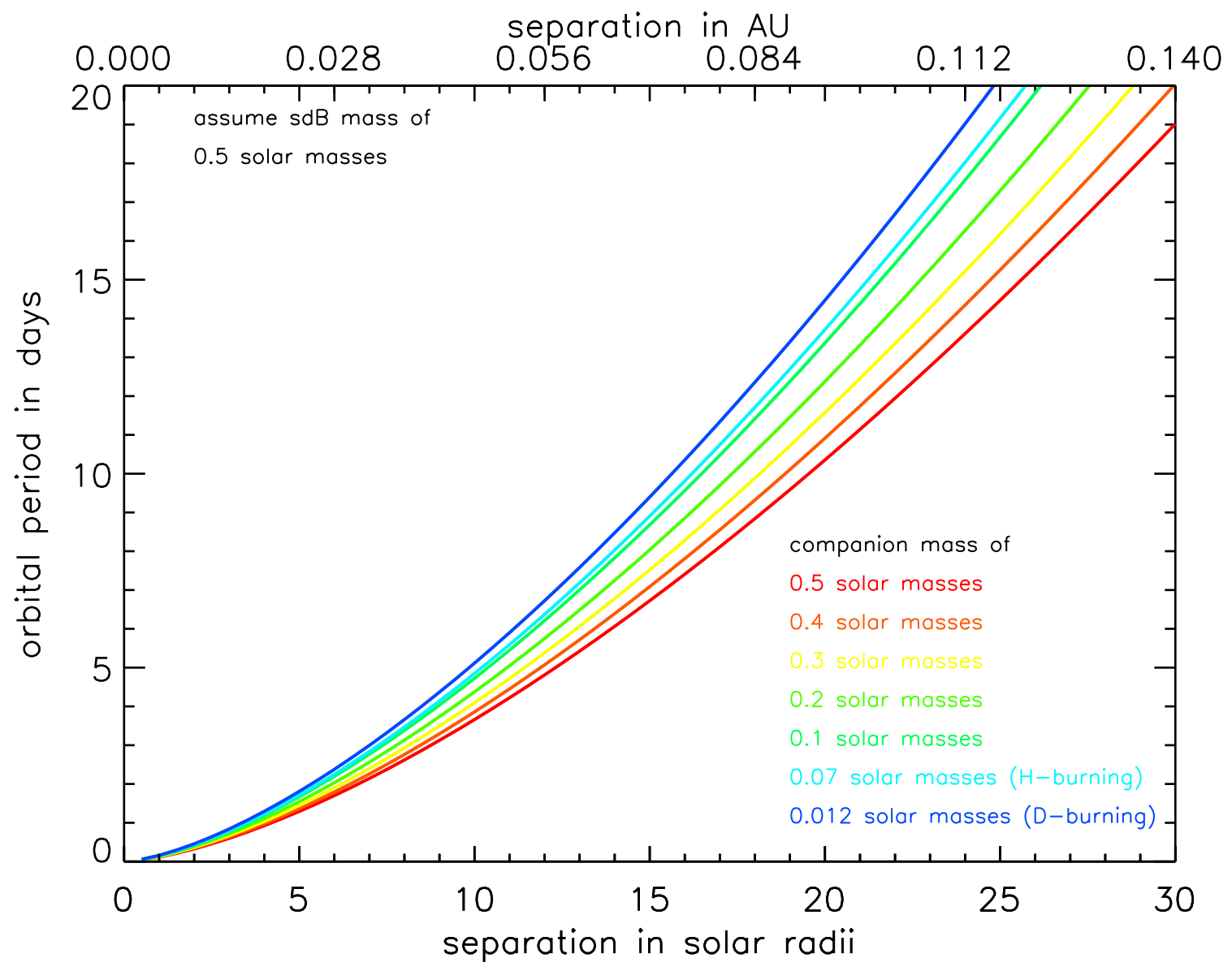

Figure 5.19: Orbital periods of hypothetical close stellar companions.

tinuous observations resulting in six $\mathrm{O}-\mathrm{C}$ points), one needs orbital periods longer than $\approx 18$ days to construct a meaningful $\mathrm{O}-\mathrm{C}$ diagram containing enough subsets, or in other words, to sample the orbit of a close-in stellar companion. A stellar companion to a sdB star with the canonical mass of half a Solar mass has orbital periods $P_{\text {orb }}>18$ days for approximately $a>25-30 R_{\odot}$ (depending on the companion mass), as can be estimated from Fig. 5.19, For these $a>25-30 R_{\odot}$ separations all stellar companions would produce a sdB wobble translating to an $\mathrm{O}-\mathrm{C}$ amplitude well above $8 \mathrm{~s}$ (see Fig. (5.18), which is above the sensitivity of the timing method applied to sdB pulsations.

As a remark concerning the viability of the observational rhythm being subject to these estimations, I want to point out that a realization is most probable given a fleet of several telescopes at suitable locations. Continuous 


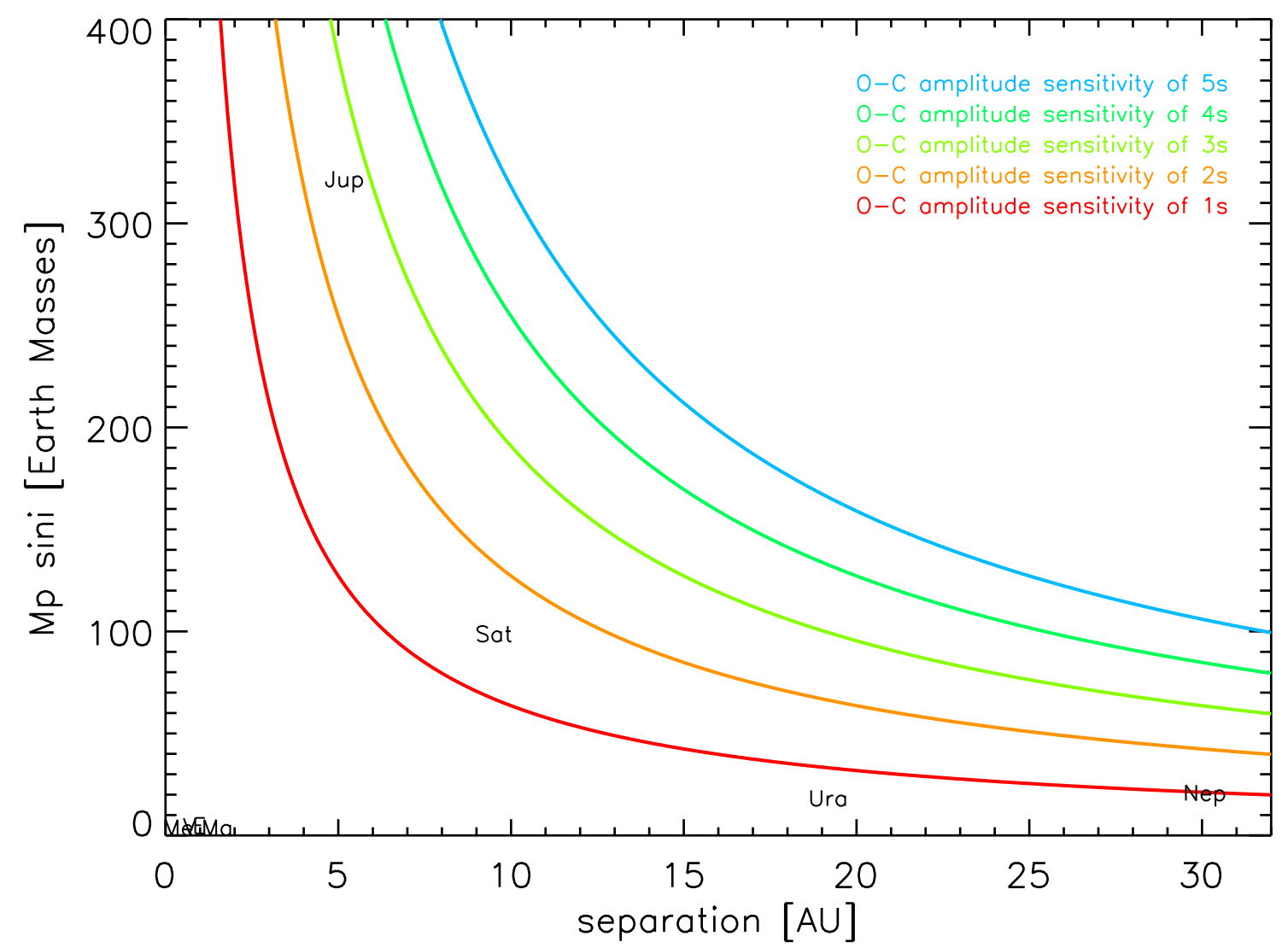

Figure 5.20: The timing method applied to our Solar System. As discussed in the text, the giant planets Jupiter, Saturn, Uranus and Neptune are close to the detection limits. The terrestrial planets (located in the very lower left of the plot), however, are well below the detection threshold.

ground based observations may be optimistic but nevertheless it is feasible within a multi-site campaign.

In conclusion, the sdB pulsation timing could detect all stellar companions to sdB stars for orbital separations larger than $\approx 25-30$ Solar radii $(\approx 0.12-$ $0.14 \mathrm{AU})$ given an observational data set as described above. For separations smaller than 25-30 Solar radii the amplitude signals could still be large enough to be detected, but the orbital periods become too short to resolve a full orbit in the $\mathrm{O}-\mathrm{C}$ diagram by means of typical ground based observations. In this context, I want to mention the case of the pulsating sdB star Feige 48. The noisy behavior in the $\mathrm{O}-\mathrm{C}$ diagram of this object (Reed et al., 2004) was later (based on RV measurements) explained by the presence of a close 
white dwarf companion in an orbit of only 0.376 days (O'Toole et al., 2004). At such a close distance, due to the low $\mathrm{O}-\mathrm{C}$ amplitude and short orbital period, the companion would not be detectable via sdB pulsation timing. In addition to these estimations of the timing effect of close-in stellar companions, I also want to refer to Figure 3.5 for the IR effect of stellar companions.

We finally show the stellar wobble induced by planets applied to the Sun and our Solar System. As can be seen, the Solar System giant planets induce light time effects of the order of roughly one to three seconds. If the sun exhibited coherent pulsations (which is a prerequisite for a timing method as we apply it) an observer outside the Solar System, search for a light time effect, would probably be able to detect Jupiter and Saturn or even Uranus and Neptune. The terrestrial planets would however stay invisible. This of course only holds if the observer outside the Solar System would have the same observational conditions and sensitivity (i.e. techniques) as we have.

To complete this chapter I want to refer the reader to Silvotti et al. (2011), who study the potential of data taken by the CoRoT and Kepler space missions in the context of a timing analysis. The authors also test the applicability of pulsation timing to $\delta$ Scuti, $\beta$ Cephei and RR Lyrae stars. 


\section{Chapter 6}

\section{Analysis and results: HS 0444+0458 and HS 0702+6043}

In the following chapter I will present the results of our application of the timing method to the targets HS $0444+0458$ and HS 0702+6043. Major parts of this chapter will consist of the construction and interpretation of the $\mathrm{O}-\mathrm{C}$ diagrams and the disentanglement of various components. Prior to this, I will expand on the fourier analysis and some coherence tests, which were conducted to ensure that the above mentioned targets are indeed suitable for a timing method as we intend to apply.

Following a tabular summary of all important results, this chapter will close with a short statement on the current status of the analysis of the other EXOTIME targets HS 2201+2610, PG 1325+101 and EC 09582-1137.

\subsection{Light curves and periodograms}

To analyze our targets HS $0444+0458$ and HS $0702+6043$ with the timing method outlined in the previous chapter, we use photometric data spanning several years. In all following $\mathrm{O}-\mathrm{C}$ diagrams the total time base includes all data from August 2008 to November 2010 in the case of HS 0444+0458 and from November 2007 to November 2010 in the case of HS 0702+6043 (cf. Tables 4.3 to 4.9 in the data archive).

For the joined light curves of all our data within the timespans mentioned above, we calculated the full periodograms visualized in Figure 6.1. The construction of all $\mathrm{O}-\mathrm{C}$ diagrams will be carried out independently for the two strongest frequencies in each target. We derive the following frequencies 

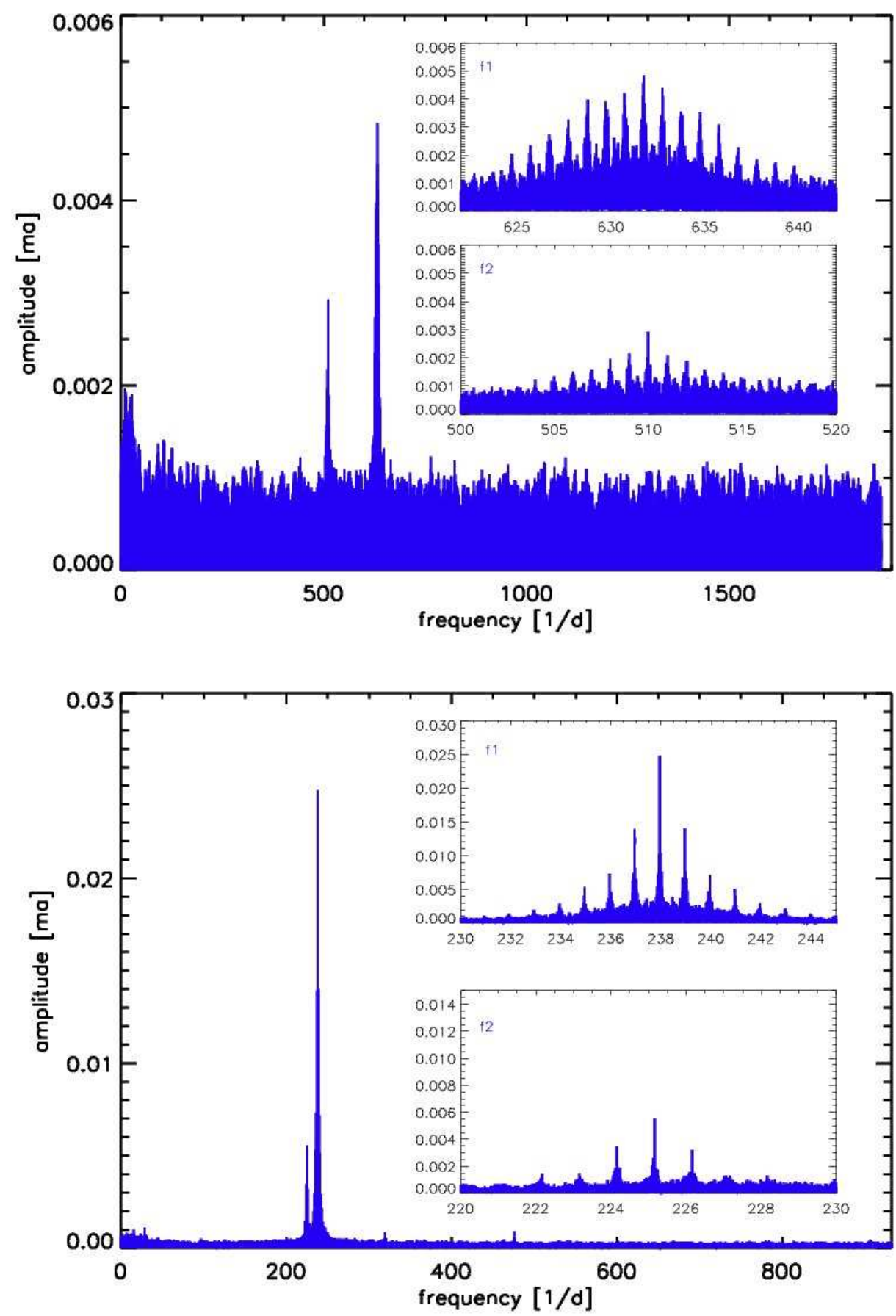

Figure 6.1: Periodograms for HS 0444+0458 (top) and HS0702 +6043 (bottom) based on our total data sets. The full frequency range up to the Nyquist frequency is plotted. The inset windows show a zoom on the two strongest frequencies $\mathrm{f} 1$ and $\mathrm{f} 2 \mathrm{in}$ each target, which are used for the independent construction of $\mathrm{O}-\mathrm{C}$ diagrams (see text for further details). 
(in $1 / \mathrm{d}$ ), periods (in s) and amplitudes (in milli modulation amplitudes, mma, $1 \mathrm{mma}=0.1 \%$ ):

- HS $0444+0458$

- f1: $631.73495(1) \frac{1}{d}, \quad 136.766(1) \mathrm{s}, \quad 4.84(13) \mathrm{mma}$

- f2: $509.97773(1) \frac{1}{d}, \quad 169.419(1) \mathrm{s}, \quad 2.92(13) \mathrm{mma}$

- HS $0702+6043$

- f1: 237.94106(1) $\frac{1}{d}, \quad 363.115(1) \mathrm{s}, \quad 24.77(03) \mathrm{mma}$

- f2: 225.15882(1) $\frac{1}{d}, \quad 383.729(1) \mathrm{s}, \quad 5.55(03) \mathrm{mma}$

Some example light curve chunks of our targets can be found in the appendix for some observational sites to compare visually the different qualities. In the appendix, I also present some example Fourier transforms of different temporal resolutions in order to illustrate that at least three consecutive nights of data are necessary to achieve a well enough frequency resolution in order to proceed in constructing a reliable $\mathrm{O}-\mathrm{C}$ point.

During the analysis of the light curves we also conducted a full pre-whitening of the total data sets to learn more about the overall frequency content of our targets. It was previously known that HS 0702+6043 shows peaks in the $g$-mode regime (see e.g. Schuh et al.. 2006: Lutz et al... 2008: Francoeur et al., 2010) and we can confirm these previous studies. Even more, our year-long data sets provide a much better frequency resolution as well as a lower noise level than the studies above. In summary, there are much more frequencies present in HS 0702+6043 than previously thought, both in the $p$ - and $g$-mode regimes. If we just count the bare number of peaks above the noise, without asking if they are intrinsic to the star, there is still significant residual power left even after $>100$ pre-whitening steps. However, a full frequency analysis is not intended to be done within the course of this thesis. The high quality Mt. Bigelow campaign on HS 0702+6043 (see Table 4.3) was done with the intention to provide a solid backbone for an asteroseismic analysis. A frequency list based on that campaign can be found in Francoeur et al. (2010). The photometric data of the Mt. Bigelow runs were kindly provided to us by E.M. Green (priv.comm.) to include them in 
our $\mathrm{O}-\mathrm{C}$ analysis.

Concerning HS $0444+0458$, the pre-whitening reveals $>20$ peaks above the noise. Again, we do not analyze these in greater detail since this is not the topic of this thesis. However, keeping in mind that early results of the Kepler space mission suggest that most of the pulsating sdBs in the Kepler field-of-view are probably hybrid pulsators (Reed et al., 2010), the $g$-mode frequency regime of HS $0444+0458$ is worth a further study.

I want to emphasize that, although our frequency analyses with the full prewhitening of the total data sets detect much more than two frequencies, we can only use the two strongest pulsations in each target to perform a reliable $\mathrm{O}-\mathrm{C}$ analysis. This is because of the much poorer frequency resolution and higher noise level of the seasonal data subsets compared to the full data sets. Only the two strongest frequencies in each target can be resolved and determined unambiguously in every single seasonal data subset. The procedure was therefore as follows. For both targets we performed a full pre-whitening based on the total data sets over the whole frequency range ${ }^{1}$ and down to the noise in order to determine the residuals. This step was part of the weighting procedure. The same process for the full data set was done again (now with the weighted light curves) in order to determine the "C"-part, i. e. the model for each of the two strongest pulsations in HS 0444+0458 and HS 0702+6043 (as listed above). With these frequencies now kept fixed, we analyzed the seasonal data subsets and redetermined the phases which were subsequently used to derive the phase differences and, based on that, the time differences and hence the $\mathrm{O}-\mathrm{C}$.

When fitting the fixed frequencies from the pre-whitening to the seasonal data subsets to determine the phases of $\mathrm{f} 1$ and $\mathrm{f} 2$ in both targets, we always tried to fit as many frequencies as possible ${ }^{2}$, but at least always the two strongest ones.

\footnotetext{
${ }^{1}$ Up to the Nyquist frequency.

${ }^{2}$ In this context one has to be cautious not to try to fit two individual frequencies which are less separated than the actual frequency resolution of the seasonal data subset.
} 


\subsection{Coherence tests}

We have already pointed out earlier that it is crucial for the application of the timing method that the phases of the pulsations are stable. In this context, by stable we mean that severe phase jumps do not occur on short timescales. Short term coherence tests for both targets have been conducted by us with the result that there are no significant phase irregularities and therefore both stars are suitable for a long-term monitoring aiming at the measurement of secular period or evolutionary changes. We present such coherence tests based on six nights of TNG 3.6m data (HS 0444+0458, 1928 August 2008) and eight nights of Calar Alto 2.2m data (HS 0702+6043, 17-27 January 2005) in Schuh et al. (2010). Additionally, I want to refer to Löptien (2009) who used a subset of the HS 0444+0458 data presented here to analyze the pulsational spectrum and also addressed aspects of short-term coherence and mid- to long-term behavior of the pulsational periods.

\section{$6.3 \mathrm{O}-\mathrm{C}$ diagrams}

In the following I want to focus on the interpretation of the $\mathrm{O}-\mathrm{C}$ diagrams. The basic concept of the construction and the mathematical background was provided in the previous chapters.

As mentioned before, I will present independent $\mathrm{O}-\mathrm{C}$ analyses based on the two strongest frequencies f1 and f2 in each of our targets, HS 0444+0458 and HS $0702+6043$, in this thesis. It is desirable to have at least two independent frequencies since this provides a way to confirm or reject certain results, since some effects must be seen in all frequencies the same way. The attempt to construct $\mathrm{O}-\mathrm{C}$ diagrams for more than the two strongest frequencies failed due to the very low amplitudes of these, resulting in huge error bars and therefore the $\mathrm{O}-\mathrm{C}$ diagrams were not meaningful. The same is unfortunately true for an attempt to construct an $\mathrm{O}-\mathrm{C}$ diagram based on a $g$-mode in HS $0702+6043$. We are thus limited to the two strongest frequencies in our targets, respectively.

For the construction of the single seasonal $\mathrm{O}-\mathrm{C}$ points we tried, if possible, 
to use only the data from the same observational site. Since this was not always the case we applied the weighting scheme described earlier. In most cases we combined all data within a month to a seasonal data point and we avoided to join data with larger gaps in between them (cf. Figures 4.3 and 4.4 for the temporal distribution of our observations). In some cases, when a lot of data are present during one month (e.g. in the time-span November 2007 - March 2008 for HS 0702+6043) we were able to derive two or even three $\mathrm{O}-\mathrm{C}$ points per month.

A binning of several $\mathrm{O}-\mathrm{C}$ points on one hand reduces the error bars but on the other hand results in larger errors in the temporal direction. Since we want to get as many $\mathrm{O}-\mathrm{C}$ points out of our data as possible we chose not to bin these. The final temporal coordinate of any $\mathrm{O}-\mathrm{C}$ point is calculated as the midpoint between the first and last exposure of the data comprising a seasonal subset. In total, there are $12 \mathrm{O}-\mathrm{C}$ points for HS $0444+0458$ from August 2008 until November 2010 and 29 O-C points for HS 0702+6043 from November 2007 until November 2010. Each of these points is, as already stated earlier, calculated on the basis of several single light curves joined together.

As already outlined before, during the re-calculation of the phases of the seasonal subsets, we tried to fit as many frequencies (which result from the total data set) as possible to the subsets. Fitting the two strongest frequencies was always possible. However, in some cases it was not possible to fit many more frequencies in the subsets, although these were clearly present in the total data set. The number of frequencies used in the subsets of HS 0702+6043 was between two (in the shorter subsets with lower quality) and six (in the longer subsets with higher quality). Due to the lower amplitudes in HS $0444+0458$ only two frequencies were used in all of the subsets concerning this target. For HS 0702+6043 we also checked the influence on the results for the two cases of using only two frequencies in all subsets compared to using as many frequencies as possible in the subsets. We found no significant difference but decided the latter case to be more reliable/realistic.

To exclude systematic errors due to possible time inaccuracies between different observing sites, we cross-checked our 'C'-part solution, i.e. the model 
(frequencies, amplitudes and phases) based on the total data set, with a model containing the same data as above, but with the data of one certain observatory excluded. This has been done separately for all observing sites with the result that there do not appear any inaccuracies or systematic errors in the frequencies, amplitudes or phases. We can therefore assume that there are no time inconsistencies between different observatories.

Let us recall possible components or signatures which might appear in an O-C diagram:

- Evolutionary signals have a parabolic shape. We use Equation 5.10, $O-C=\Delta T_{0}+\frac{\Delta P}{P} t+\frac{1}{2} \frac{\dot{P}}{P} t^{2}$, to fit these and to calculate $\dot{P}$.

- Orbital signals are represented by sinusoidal (in case of circular orbits) signatures in the $\mathrm{O}-\mathrm{C}$ diagram. They are fitted using the equation

$$
O-C=\tau \cdot \sin \left(\frac{2 \pi}{P_{\text {orb }}} t+\Phi\right)
$$

with $\tau, P_{\text {orb }}$ and $\Phi$ being the $\mathrm{O}-\mathrm{C}$ amplitude (in sec), the orbital period (in days) and the orbital phase.

- Beating signals are also related to sinusoidal $\mathrm{O}-\mathrm{C}$ variations and are fitted with the same equation as above, just with the orbital period to be replaced with the beat period. A detailed description of the beating is presented in Chapter 6.3.2.

- Since all the above signatures may be present in the $\mathrm{O}-\mathrm{C}$ diagram at the same time, we fit all possible signatures simultaneously with the following equation:

$$
O-C=\Delta T_{0}+\frac{\Delta P}{P} t+\frac{1}{2} \frac{\dot{P}}{P} t^{2}+\sum_{j} \tau_{j} \cdot \sin \left(\frac{2 \pi}{P_{o r b, j}} t+\Phi_{j}\right)
$$

Here, $j$ can stand for beating- or orbital signals. In the former case the beat period has to be used. In both cases we restrict the signal to be strictly sinusoidal, which in case of the orbital signals means a restriction to circular orbits. 
The error bars for each single $\mathrm{O}-\mathrm{C}$ point in our $\mathrm{O}-\mathrm{C}$ diagrams are calculated according to

$$
\sigma_{\tau}=\frac{P}{2 \pi} \sigma_{\phi}=\frac{P}{2 \pi} \frac{\sigma_{A}}{A}
$$

with $P$ being the period of the pulsation and $A, \sigma_{A}$ and $\sigma_{\phi}$ as defined in equations 5.3 and 5.4 .

As can be seen, the error in the timing is proportional to $P / A$. The minimum detectable mass with the timing method of course also depends on $\sigma_{\tau}$. Given this, the timing error and therefore also the minimum detectable mass is smaller for shorter $P$ and larger $A$ of the pulsations. As seen in equation 6.3 the timing error $\sigma_{\tau}$ also depends on the amplitude error $\sigma_{A}$.

For the non-linear least-squares curve fitting of our $\mathrm{O}-\mathrm{C}$ diagrams we use the IDL-based routine MPFIT ${ }^{3}$ (Markwardt, 2009) which uses the LevenbergMarquardt technique to solve the least-squares problem. MPFIT also computes errors for all fit quantities. To implement equation 6.2 in the above routine, we made use of a helpful tutorial ${ }^{4}$.

\subsubsection{Evolutionary signals}

As discussed in the previous chapter, evolutionary signals can be interpreted with a linearly changing period which manifests itself in the $\mathrm{O}-\mathrm{C}$ diagram by a parabolic shape. This can subsequently be used to derive a value for the secular change of the period, $\dot{P}$, and hence the evolutionary time scale. Figures 6.2 and 6.3 show the full $\mathrm{O}-\mathrm{C}$ diagrams of our targets ${ }^{5}$. The parabolic component in each plot is indicated by a green dashed line. According to equation 5.12, we derive the following secular increases in the main pulsation periods of our targets:

- HS 0444+0458 f1: $\dot{P}=(9.300 \pm 0.023) \cdot 10^{-12} \mathrm{~d} / \mathrm{d}$

- HS 0444+0458 f2: $\dot{P}=(3.010 \pm 0.010) \cdot 10^{-12} \mathrm{~d} / \mathrm{d}$

\footnotetext{
${ }^{3}$ http://purl.com/net/mpfit

${ }^{4}$ http://cow.physics.wisc.edu/ ${ }^{\sim}$ craigm/idl/mpfittut.html.

${ }^{5}$ In all figures and tables in this chapter, the BJD is shown in reduced format, i. e. the two leading digits $24 \ldots$ are cut off.
} 

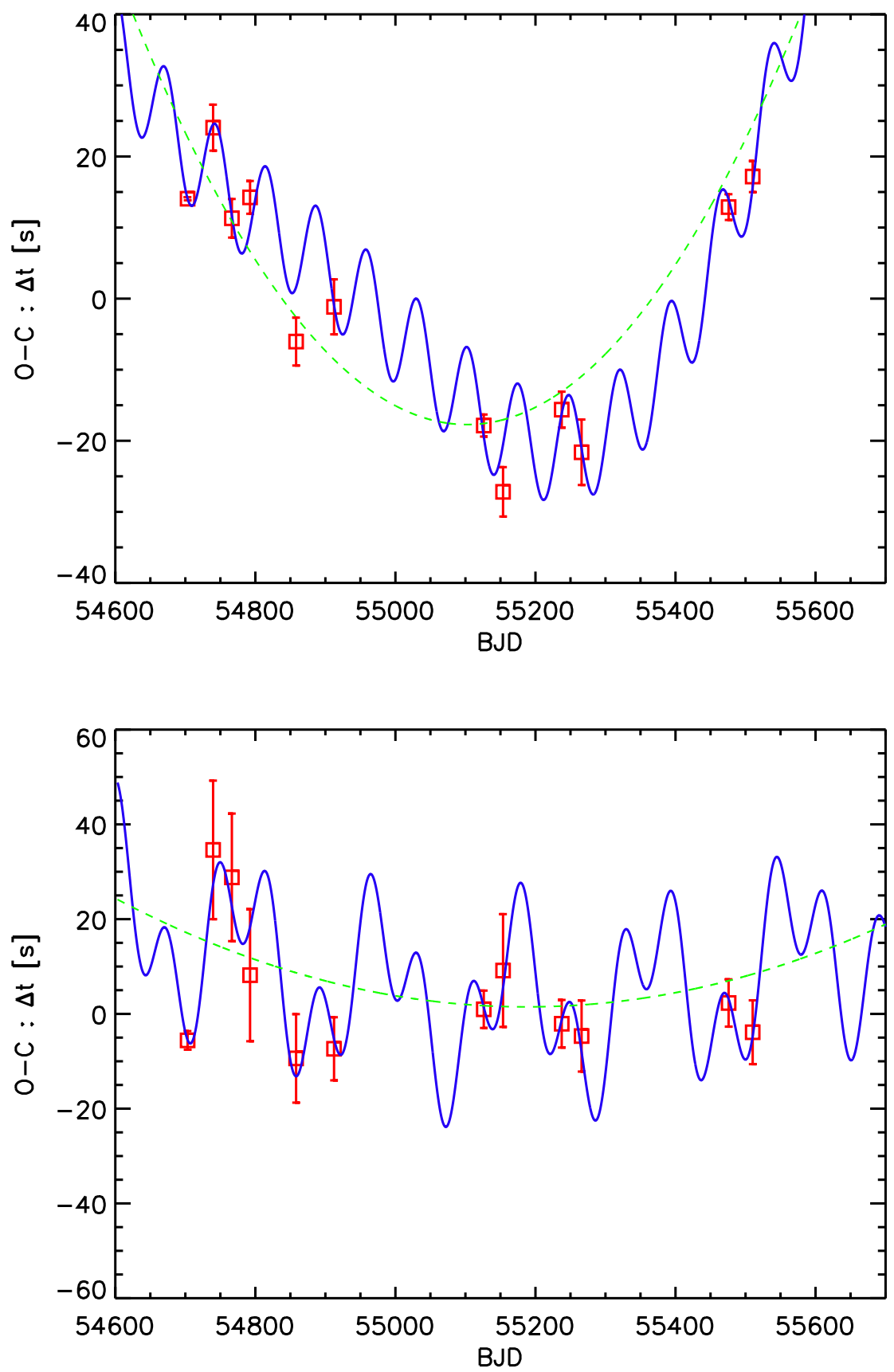

Figure 6.2: Full O-C diagrams for HS $0444+0458$ for f1 (top) and f2 (bottom). The red squares with error bars are the measurements and the green dashed line is the quadratic (evolutionary) component. The solid blue line is the best fit including one quadratic and two sinusoidal components. The time is given in barycentric Julian date (precisely BJD $\mathrm{TT}-65.184 \mathrm{~s}$, see Figure 5.5) . 

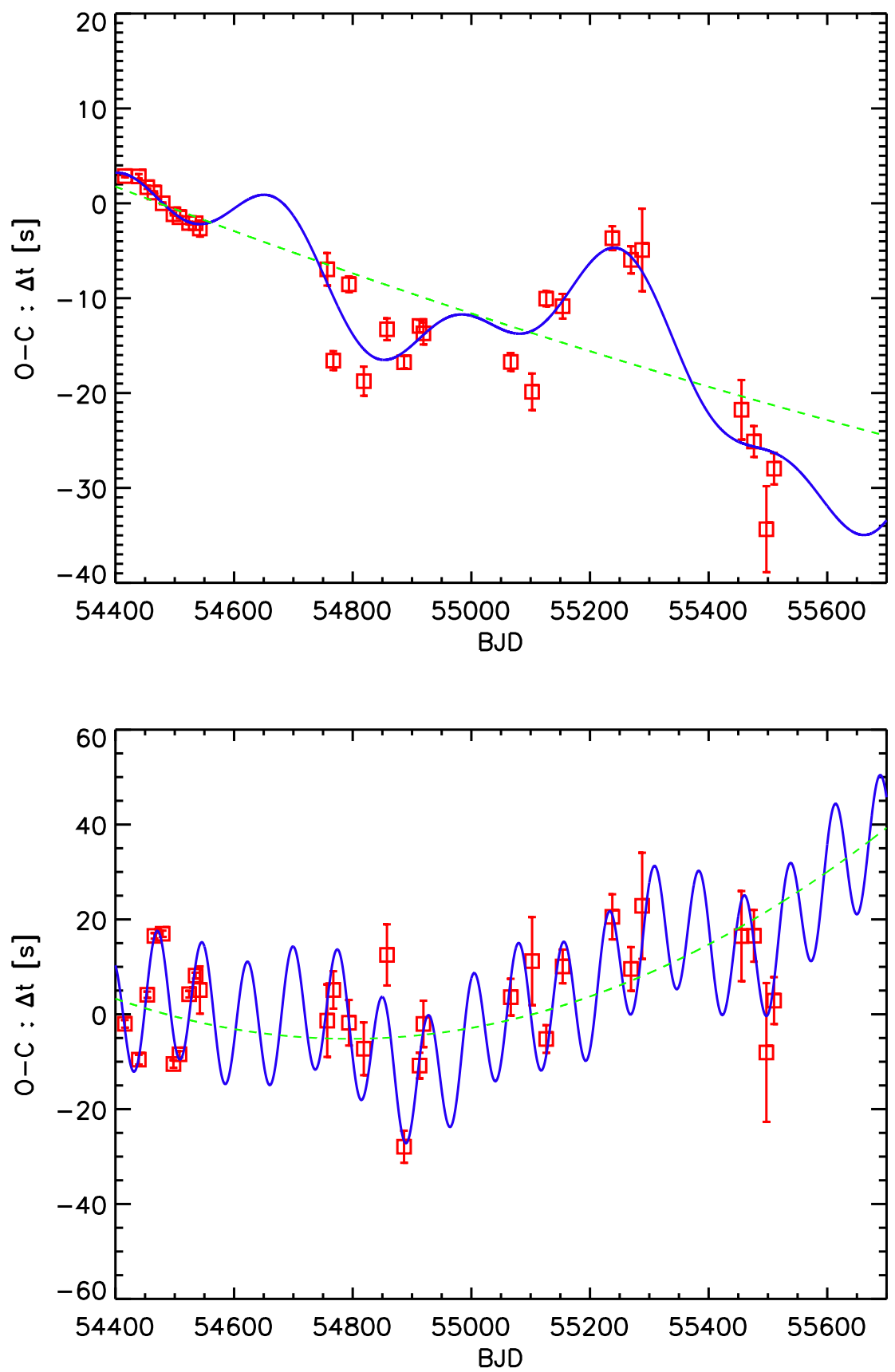

Figure 6.3: Full O-C diagrams for HS 0702+6043 for f1 (top) and f2 (bottom). The red squares with error bars are the measurements and the green dashed line is the quadratic (evolutionary) component. The solid blue line is the best fit including one quadratic and three sinusoidal components. The time is given in barycentric

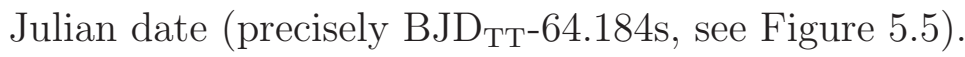


- HS 0702+6043 f1: $\dot{P}=(2.871 \pm 0.025) \cdot 10^{-13} \mathrm{~d} / \mathrm{d}$

- HS 0702+6043 f2: $\dot{P}=(5.578 \pm 0.006) \cdot 10^{-12} \mathrm{~d} / \mathrm{d}$

Not only evolutionary changes will have an influence on $\dot{P}$. Pajdosz (1995a, b considers the effect of proper motion as contribution to the measured $\dot{P}$. The author derives $\dot{P}_{p m}=1.081 \cdot 10^{-19} \mathrm{Pd}^{-1} v_{t}^{2}$ or $\dot{P}_{p m}=2.430 \cdot 10^{-18} P \mu^{2} \pi^{-1}$ or $\dot{P}_{p m}=2.430 \cdot 10^{-18} P \mu^{2} d$, where $\dot{P}_{p m}$ is the effect of proper motion on the rate of period change, $P$ is the period in seconds, $v_{t}$ is the stellar total velocity in $\mathrm{km} / \mathrm{s}, \mu$ is the proper motion in arcseconds/year, $\pi$ is the parallax in $\operatorname{arcsec}$ and $d$ the distance in parsec $(\mathrm{d}[\mathrm{pc}]=1 / \pi[\operatorname{arcsec}])$.

We will now estimate the proper motion contribution on $\dot{P}$ for our targets HS 0444+0458 and HS 0702+6043. Since we do not know the exact proper motions of these two stars, we will use a conservative upper limit for $\mu$. Thejll et al. (1997) and Tillich et al. (2011) investigate the kinematic properties of some sdB samples belonging to different populations. From Tables 3 and 4 in Thejll et al. (1997) and Table 2 in Tillich et al. (2011) we regard 0.03 arcseconds per year as a conservative upper limit for the proper motion of our sdB targets. Together with the period and distance (see Table 3.1) of our targets this yields, according to the equations above, an effect of $\dot{P}_{p m, 0444}$ of the order $6-7 \cdot 10^{-16}$ (derived for both periods) and $\dot{P}_{p m, 0702}$ of the order $1.7 \cdot 10^{-15}$ (again derived for both periods). Using an even more conservative upper limit of 0.07 arcseconds per year, the proper motion contributions would be $\dot{P}_{p m, 0444} \approx 3-4 \cdot 10^{-15}$ and $\dot{P}_{p m, 0702} \approx 9.0 \cdot 10^{-15}$, respectively. This qualitative estimation shows that the proper motion contribution to the measured $\dot{P}$ is, even in a conservative upper limit, still two to three orders of magnitude smaller than the evolutionary effect on $\dot{P}$. In the following I will therefore neglect the contribution of the proper motion on $\dot{P}$.

Figures 6.4 and 6.5 illustrate the $\mathrm{O}-\mathrm{C}$ diagrams with the evolutionary signals removed.

\subsubsection{Beating signals}

If a star exhibits two pulsations with frequencies which are close to each other, these will be subject to beating. If these cannot be resolved in the Fourier spectrum, they will appear as an apparently single frequency with 

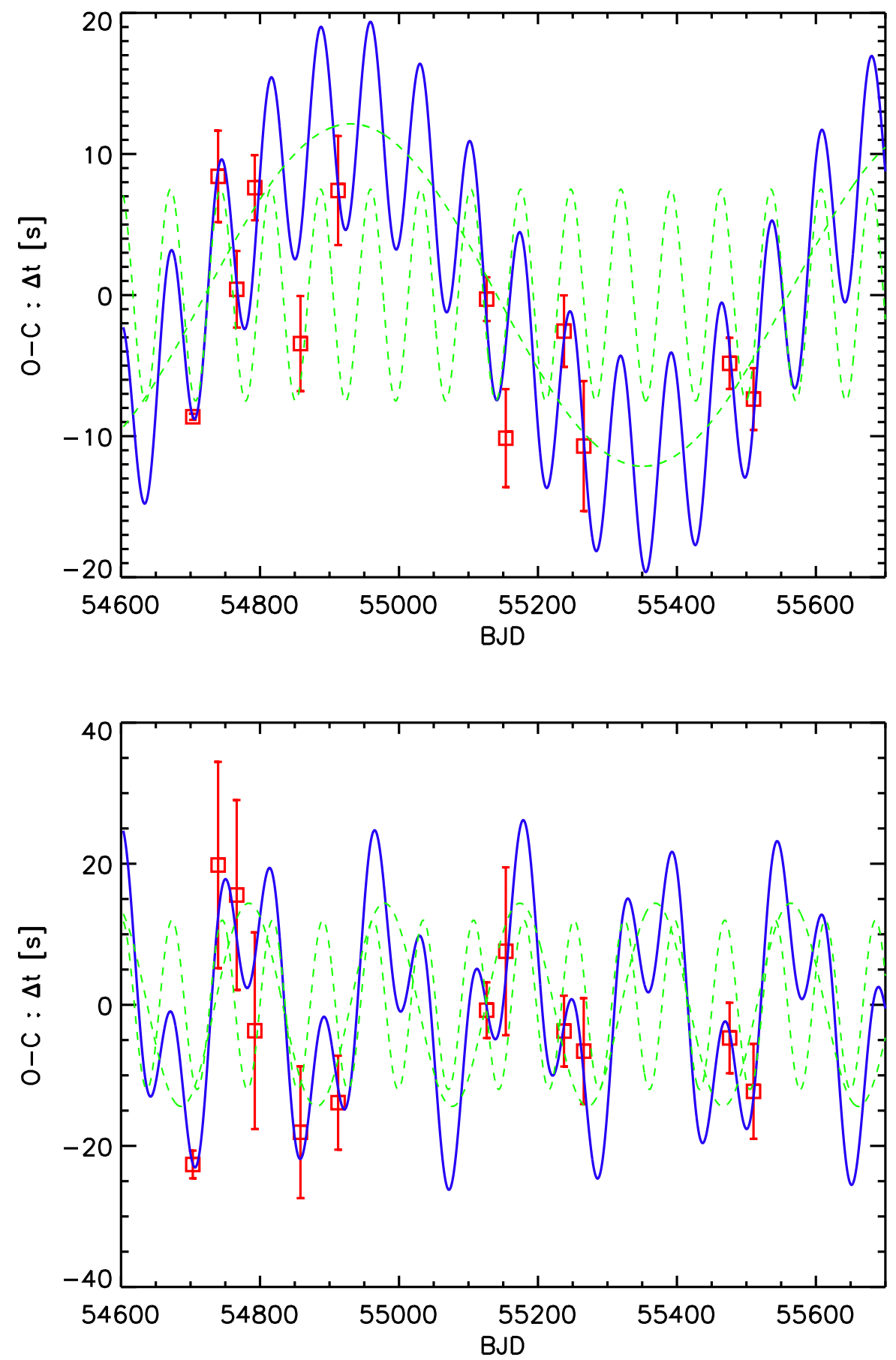

Figure 6.4: O-C diagrams for HS 0444+0458 for f1 (top) and f2 (bottom). Evolutionary signals (quadratic components) are subtracted. The solid blue line is the best fit and the green dashed lines are the two individual sinusoidal signatures. 

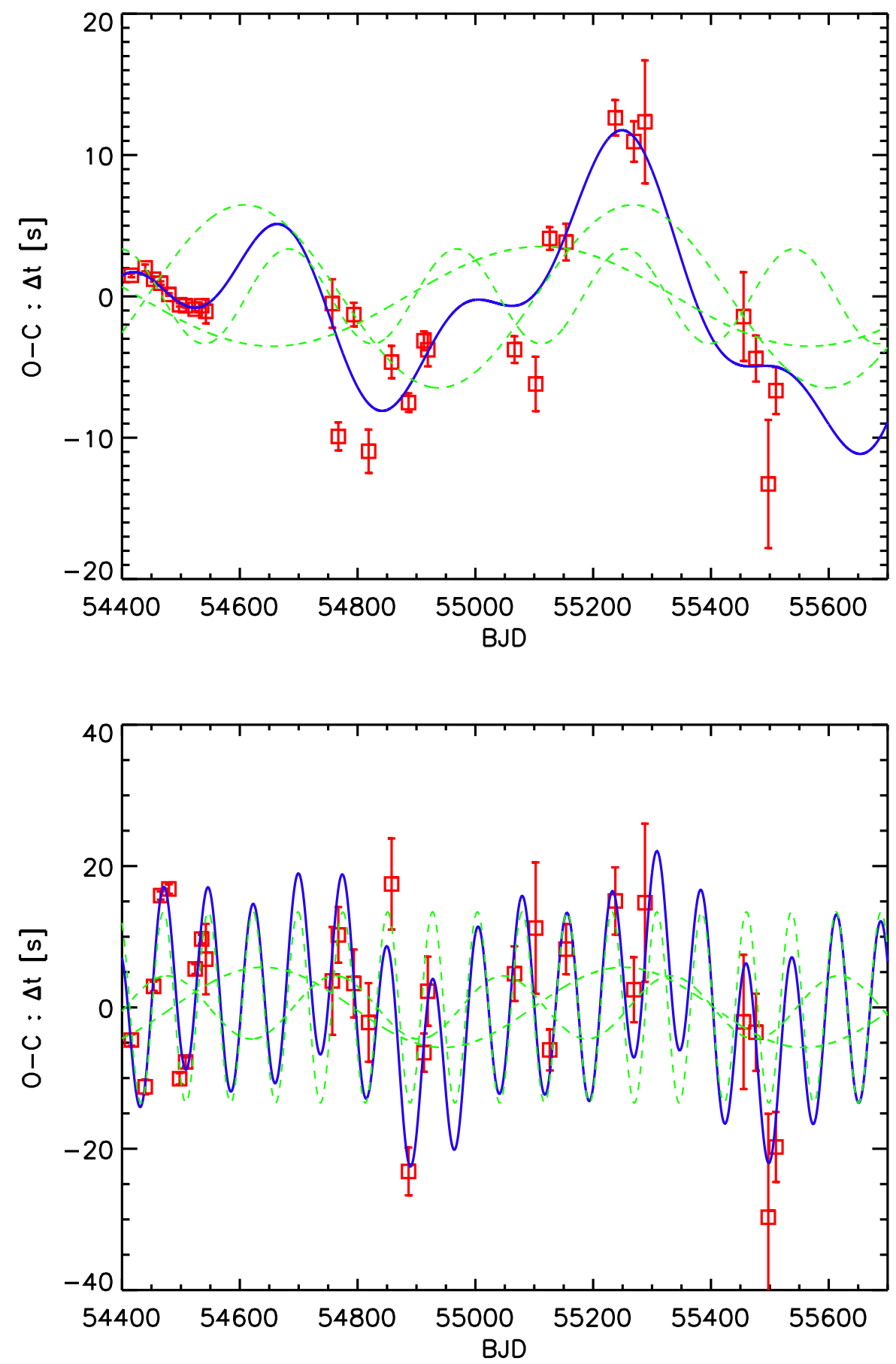

Figure 6.5: O-C diagrams for HS 0702+6043 for f1 (top) and f2 (bottom). Evolutionary signals (quadratic components) are subtracted. The solid blue line is the best fit and the green dashed lines are the three individual sinusoidal signatures. 

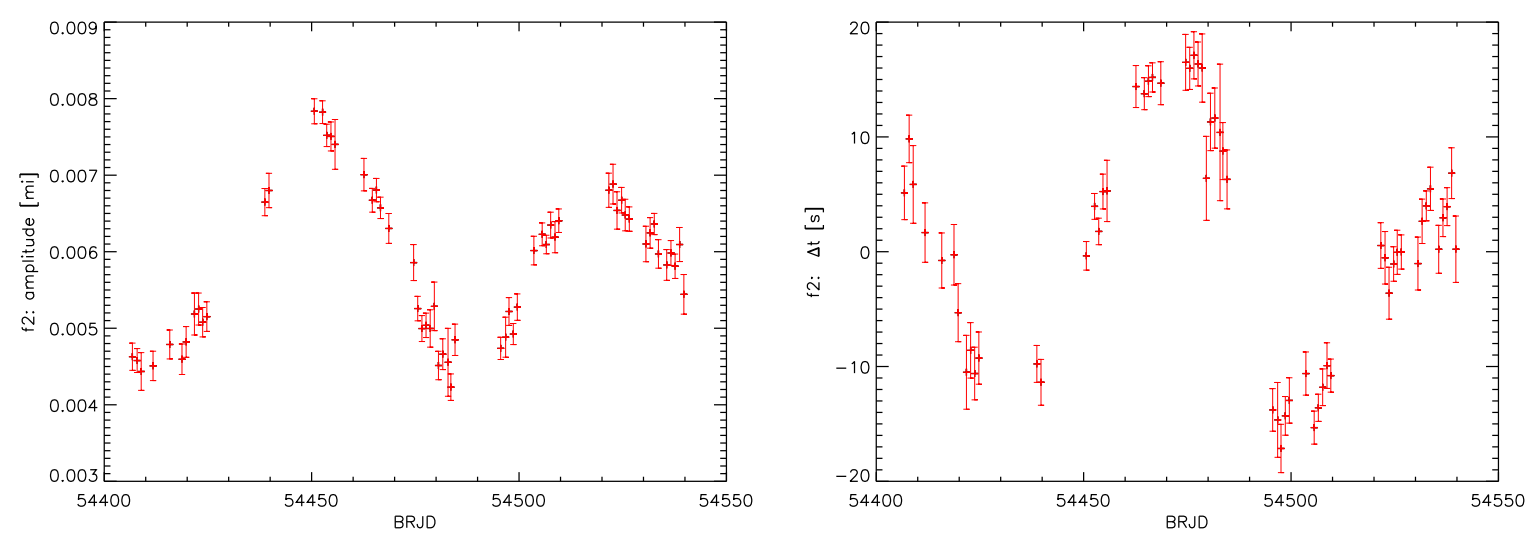

Figure 6.6: Textbook example for beating (f2 in HS 0702+6043) seen in the high quality Mt. Bigelow data. The left panel shows the amplitude variations and the right one the phase variations. Note that the signatures are shifted by $\pi / 2$, i.e. the maximum change in the phase appears at maximum/minimum amplitude. More textbook examples of beating are shown in Breger \& Pamyatnykh (2006).

variable amplitude and phase. This means that amplitude variability is not necessarily intrinsic to the mode but can also be caused by beating of two close frequencies. With the means of an $\mathrm{O}-\mathrm{C}$ analysis the beating can be revealed because the amplitude- and phase variability caused by this phenomenon is related as follows: both the amplitude- and phase variation have the same period and furthermore these two variations are shifted by $\pi / 2$ with respect to each other. This can be clearly seen in Figure 6.6, which shows a 'text-book' example for beating. Generally, the largest phase change appears at the time when the amplitude variation has a minimum or maximum. If an amplitude variability is present it is therefore possible, by looking at the phase variability, to test if this amplitude variability is due to intrinsic effects or due to beating. Since we measure amplitude variabilities in all the four frequencies we inspect (f1, f2 in HS 0444+0458 and f1, f2 in HS 0702+6043, see Figures 6.7 and 6.8), we now want to investigate the cause. Fourier transformations of these amplitude variabilities show clear peaks, meaning that the variations are not random, but periodic. And indeed, looking at the periodograms of our total light curves (left panels in Figures 6.7 and 6.8), we find significant peaks close to the respective frequencies at exactly the distances where we would expect these according to the periods measured in the amplitude variations. 

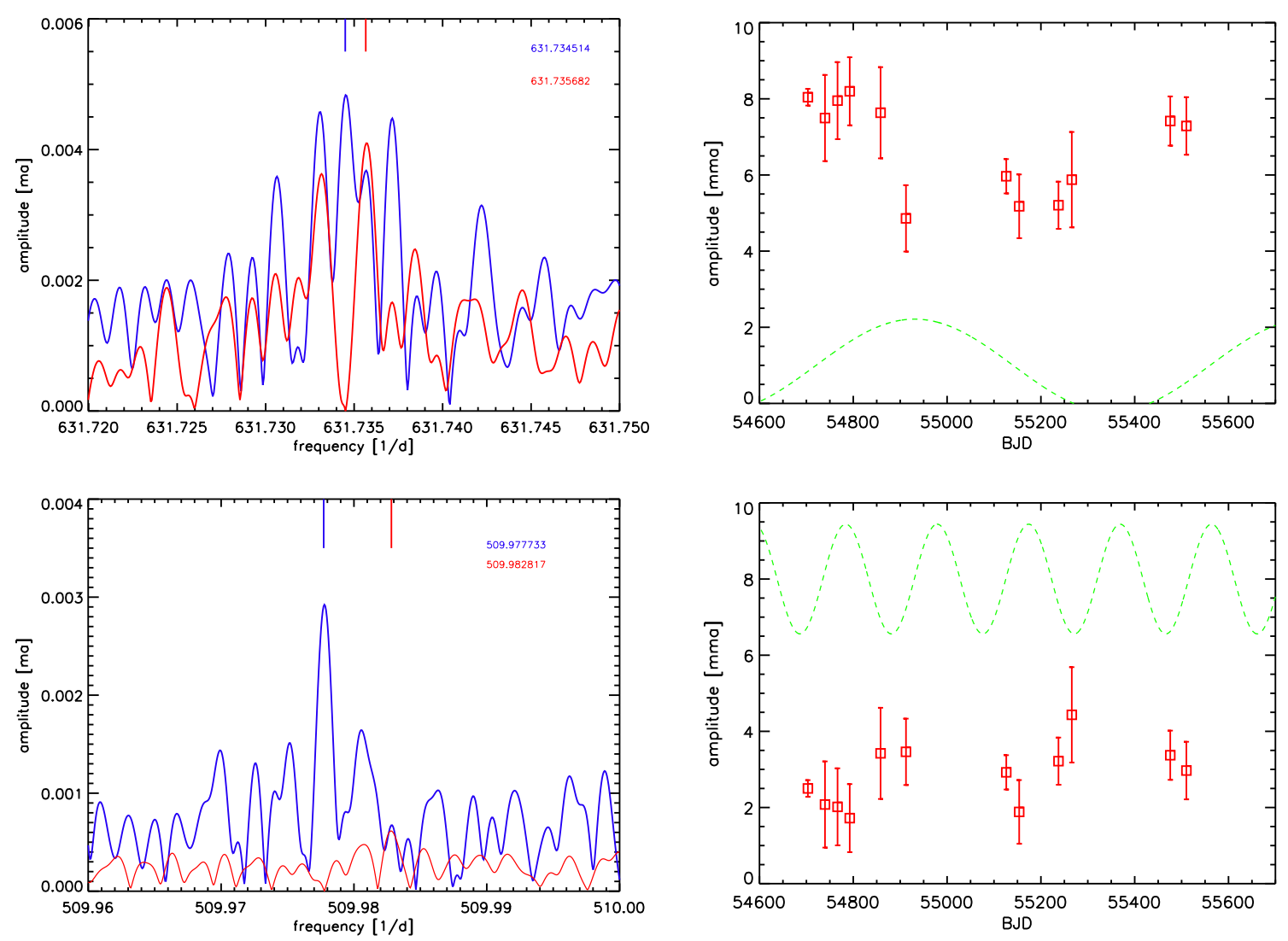

Figure 6.7: Left panels: beatings in HS $0444+0458$ as seen in the FT (top left for $\mathrm{f1}$, bottom left for $\mathrm{f2}$ ). The red solid line is the signal after pre-whitening of the main signal shown as a blue solid line. Right panels: beating signatures in the amplitude variation (red squares) versus phase variation (green dashed line) for f1 (top right) and f2 (bottom right). We calculate beat periods of 840 days and 195 days for $\mathrm{f} 1$ and $\mathrm{f} 2$, respectively. See text for further explanations.

To investigate the beating quantitatively, let us now consider two oscillations with different amplitudes but very close frequencies: $y=A_{1} \cos \left(\omega_{1} t\right)+$ $A_{2} \cos \left(\omega_{2} t\right)$ with $A_{1}>A_{2}$ and $\omega_{1} \approx \omega_{2}$. Following the superposition principle and using an trigonometric addition theorem, the beat pattern in the case where the amplitudes are different is given as

$$
y=\left(A_{1}-A_{2}\right) \cos \left(\omega_{1} t\right)+2 A_{2} \cos \left(\frac{\omega_{1}-\omega_{2}}{2} t\right) \cos \left(\frac{\omega_{1}+\omega_{2}}{2} t\right) .
$$

The second term describes a pure beating (i.e. a beating of frequencies with the same amplitudes) where the amplitude of the envelope curve varies between zero and twice the amplitude of the two single frequencies. The first 

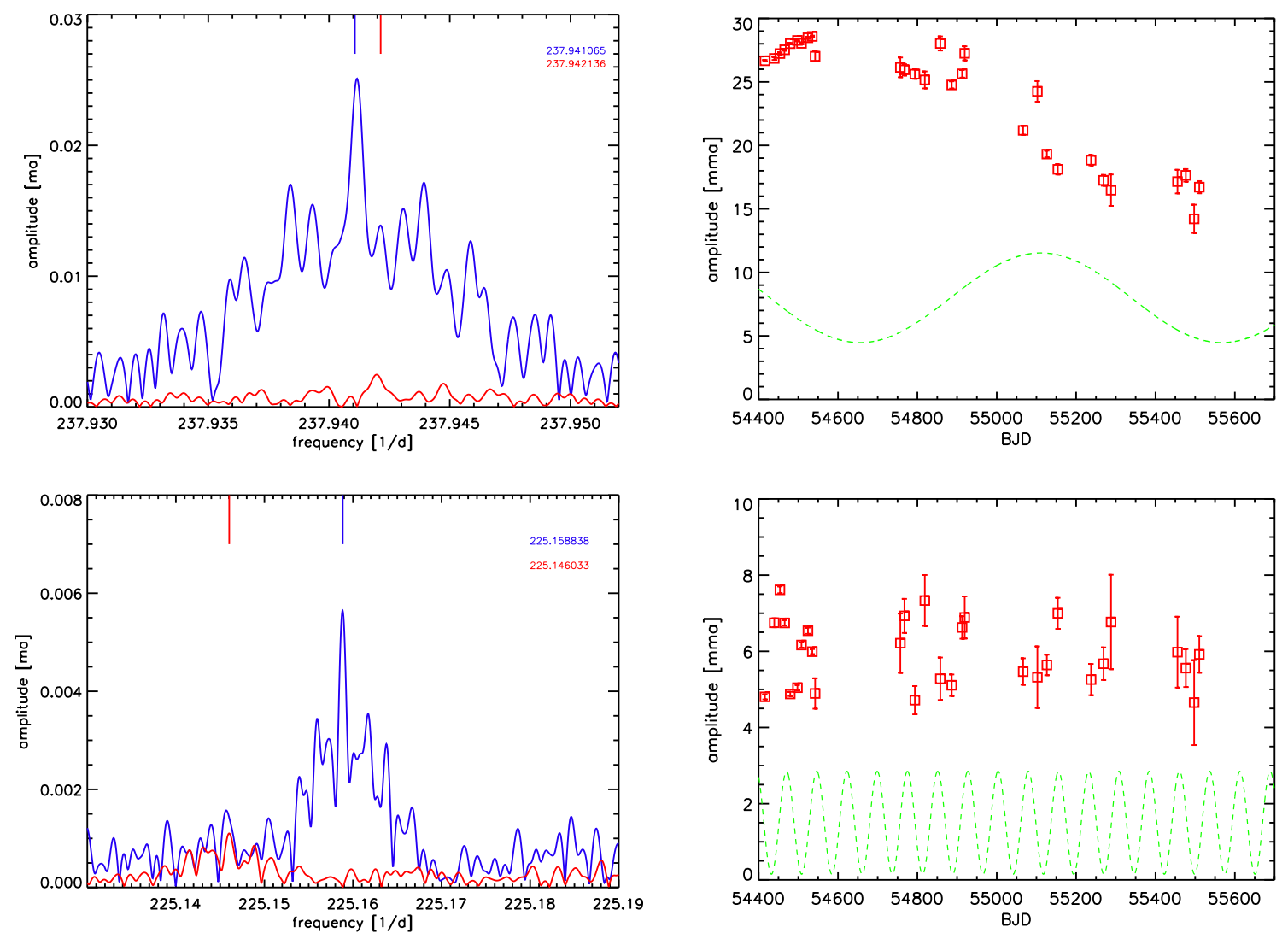

Figure 6.8: Left panels: beatings in HS 0702+6043 as seen in the FT (top left for $\mathrm{f1}$, bottom left for f2). The red solid line is the signal after pre-whitening of the main signal shown as a blue solid line. Right panels: beating signatures in the amplitude variation (red squares) versus phase variation (green dashed line) for f1 (top right) and $\mathrm{f} 2$ (bottom right). We find beat periods of 911 days and 76 days for f1 and f2, respectively. See text for further explanations.

term accounts for the situation where the two frequencies have different amplitudes. In this case the envelope amplitude varies between $\left|A_{1}-A_{2}\right|$ and $A_{1}+A_{2}$. We roughly measure the milli-modulation amplitudes $A_{1}$ and $A_{2}$ of the components which we suspect to be responsible for the beating signals in f1 and f2 of HS $0444+0458$ and $\mathrm{f} 1$ and $\mathrm{f} 2$ of HS 0702+6043, respectively, as 5 and $4 \mathrm{mma}, 2.9$ and $0.7 \mathrm{mma}, 25$ and $3 \mathrm{mma}$ and 6 and $1 \mathrm{mma}$, as can be seen in the left panels of Figures 6.7 and 6.8. We would therefore theoretically expect the envelope milli-modulation amplitude to vary between [1-9] mma, [2.2-3.6] mma, [22-28] mma and [5-7] mma, respectively. The values that we actually measure are approximately between [5-9] mma, [1.8-4.3] mma, [16-29] mma and [4.5-7.5] mma, as seen in the right panels of Figures 
6.7 and 6.8. This very good correspondence with the expected amplitude variations is a strong support for our beating hypothesis.

In order to confirm the beating hypothesis, we also looked at the phase variations. For all four frequencies considered we find a phase variation with the same period as the amplitude variation and these are indeed shifted by $\pi / 2$. We investigate this as follows: It was already pointed out before that the measured amplitude variations (the squares in the right panels of Figures 6.7 and 6.8) are not random but periodic. We calculated the period of the amplitude variability based on a Fourier analysis. In the periodograms (left panels of Figures 6.7 and 6.8) we indeed find peaks at a distance from the considered frequency which exactly transfer to the period of the amplitude variability. In the $\mathrm{O}-\mathrm{C}$ diagrams of Figures 6.4 and 6.5, in each case we detect components with exactly the same periods as the periods of the amplitude variations. To illustrate this, the corresponding phase variation found in the $\mathrm{O}-\mathrm{C}$ diagram is over-plotted as green dashed line in the plots of the amplitude variation (right panels of Figures 6.7 and 6.8). The phase variations only follow the $\mathrm{x}$-axis and are given to illustrate the $\pi / 2 \mathrm{shift}$ with respect to the amplitude variations. The y-axis is only valid for the amplitude variations. In all four cases one can see that the change in phase is most rapid at times where the amplitude variation is minimal or maximal. Based on these connections between the amplitude and phase variations and additionally strengthened by the peaks in the light curve periodograms we conclude that all amplitude variations in the considered frequencies are due to beating.

Since the two component frequencies causing the beating in all cases can only be resolved in the periodogram of our full data sets (and not in the sub-datasets used to derive the single $\mathrm{O}-\mathrm{C}$ points) we treat the beating in the $\mathrm{O}-\mathrm{C}$ diagrams and not in the light curves. In order to get rid of the beating, we subtract the phase variation belonging to the beating directly in the $\mathrm{O}-\mathrm{C}$ diagrams. To illustrate this, we have also included the beating component in the $\mathrm{O}-\mathrm{C}$ diagrams in Figures 6.4 and 6.5.

Supported by the arguments given above, we proved that beating signatures (with the characteristics as outlined before) are present in the $\mathrm{O}-\mathrm{C}$ diagrams given in Figures 6.4 and 6.5. Both the detected splittings in the Fourier 
transforms and the comparison between the amplitude and phase variations yield consistent beating signatures.

\subsubsection{Orbital signals}

Figures 6.9 and 6.10 finally show the $\mathrm{O}-\mathrm{C}$ diagrams with both the evolutionary and the beating signatures subtracted. The best fits, shown as solid blue lines in these figures, consist of one sinusoid in the case of HS 0444+0458 and two sinusoids in the case of HS $0702+6043$. These remaining sinusoidal components could possibly have an orbital motion as origin and I will now investigate these components further.

For our target HS $0444+0458$ the top panel in Figure 6.11 illustrates the sinusoidal signature derived for f1 (solid blue line) with the one derived for f2 (solid red line) over-plotted. Amplitudes and periods are consistent within the errors (cf. Table 6.2). As can clearly be seen, in addition also the phases of the orbit are consistent in $\mathrm{f} 1$ and $\mathrm{f} 2$. If a sinusoidal component is interpreted as an orbital motion, the same signatures must be present in all frequencies considered. Similarly to HS $2201+2610$, this is the case here. We measure a sinusoidal signature with the same amplitude, period and phasing independently in both frequencies under consideration. Given this, I will term this component HS $0444+0458 \mathrm{~b}$ in the following and declare it to be a companion candidate.

In the same way we investigate the sinusoidal $\mathrm{O}-\mathrm{C}$ signatures in the two frequencies of HS $0702+6043$. The first sinusoidal component is given in the middle panel of Figure 6.11, as seen in f1 (solid blue line) and in f2 (solid red line). All three parameters (amplitude, period and phasing) are consistent in $\mathrm{f} 1$ and $\mathrm{f} 2$ and derived independently, which allows to declare this component to be a companion candidate. I will term this candidate HS $0702+6043 \mathrm{~b}$ from now on.

Looking at the second sinusoidal component present in the $\mathrm{O}-\mathrm{C}$ diagrams of Figure 6.10, one finds a matching amplitude and period based on $\mathrm{f} 1$ and f2 (see bottom panel of Figure 6.11). However, the phasing is not consistent in $\mathrm{f} 1$ and $\mathrm{f} 2$, but shifted roughly by $\pi / 2$. I will term this component "HS 0702+6043 sin", but not declare it to be of orbital origin since the phasing is not the same in $\mathrm{f} 1$ and $\mathrm{f} 2$.

For the two companion candidates HS $0444+0458$ b and HS 0702+6043 b, 

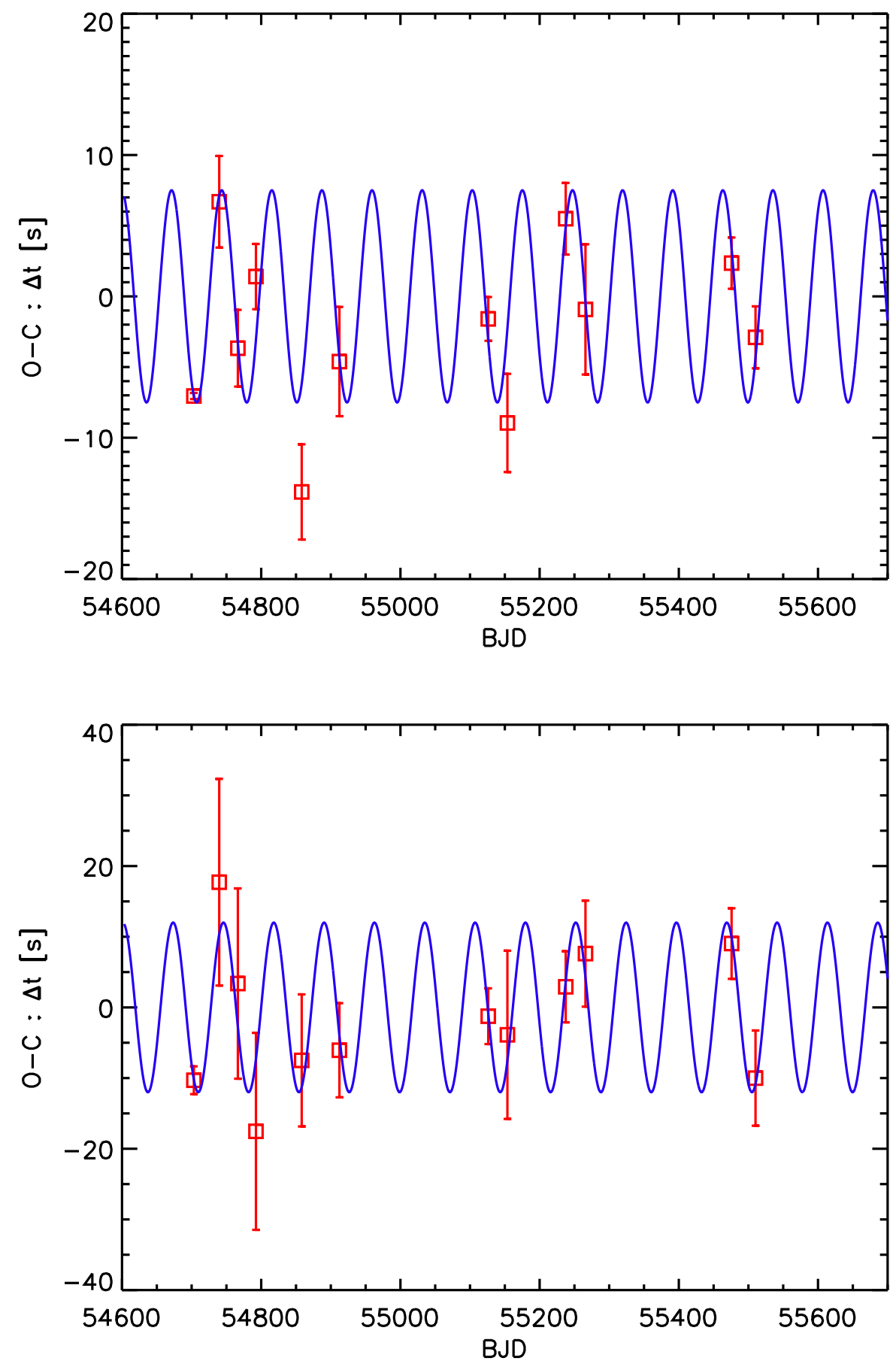

Figure 6.9: O-C diagrams for HS 0444+0458 for f1 (top) and f2 (bottom). Evolutionary signals and beating signals are subtracted. The solid blue lines are the best fits (one single sinusoid). 

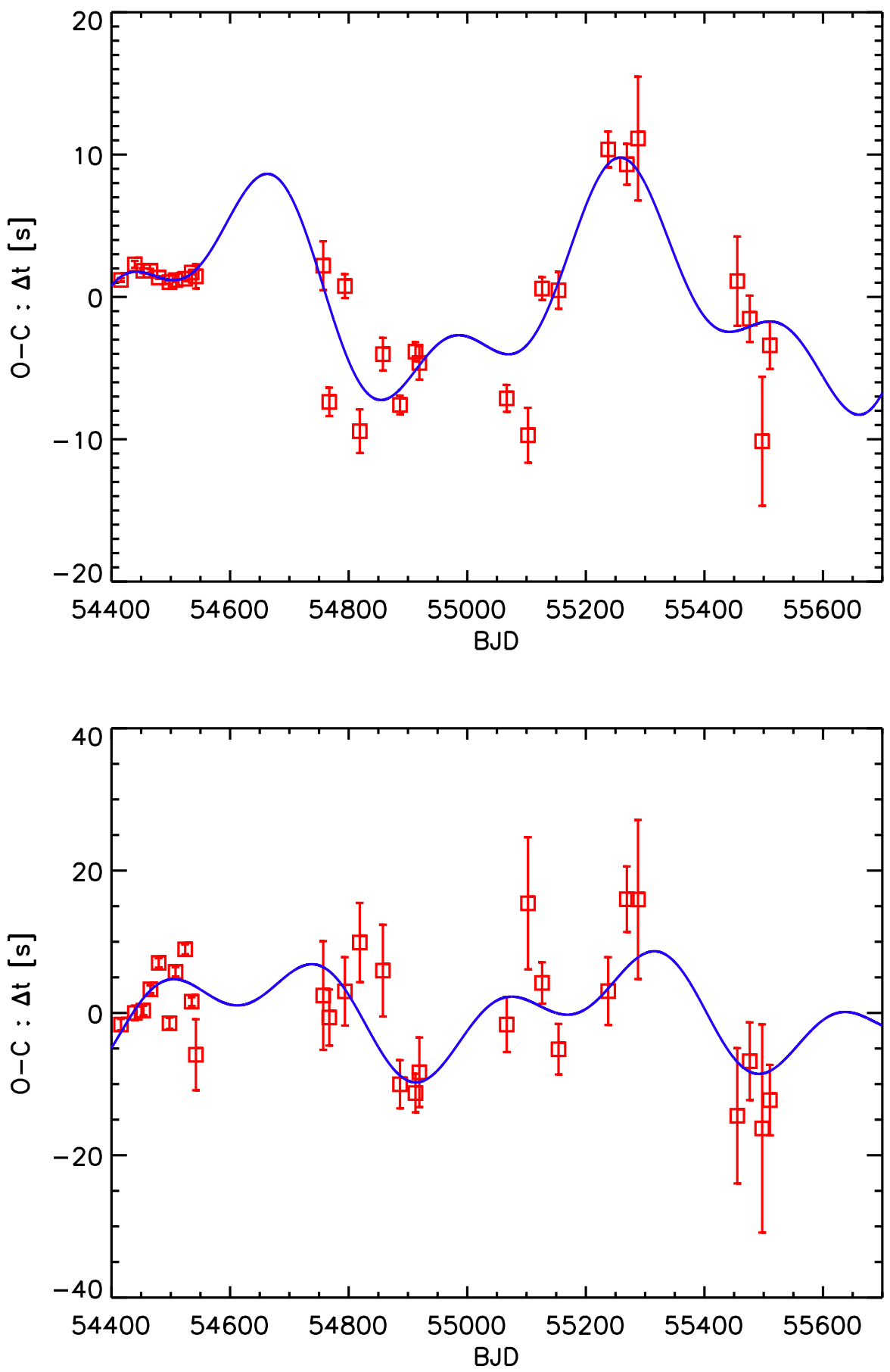

Figure 6.10: O-C diagrams for HS 0702+6043 for f1 (top) and f2 (bottom). Evolutionary signals and beating signals are subtracted. The solid blue lines are the best fits (consisting of two sinusoids). 

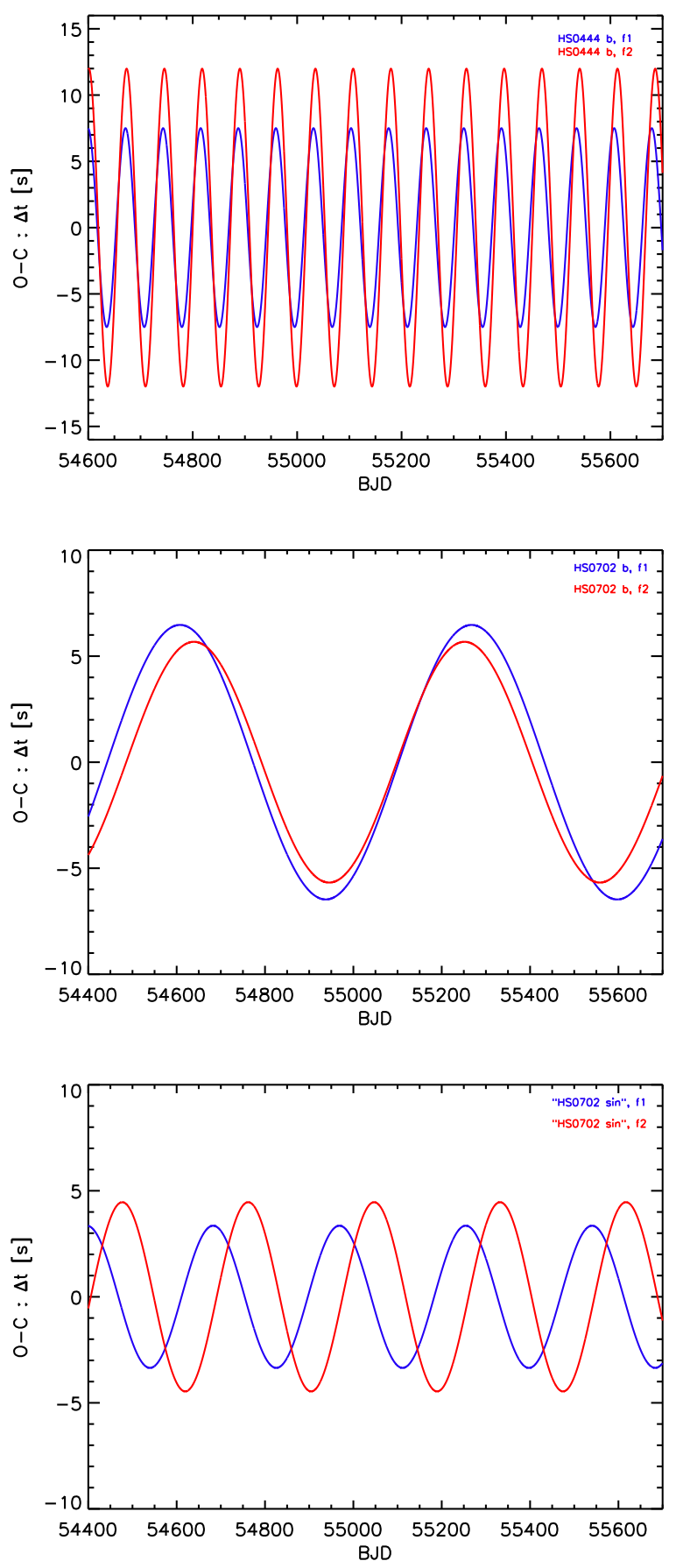

Figure 6.11: Fitted sinusoidal signatures according to f1 (blue) and f2 (red). The two upper panels show matching orbital phases. Top: candidate to HS $0444+0458$. Middle: candidate to HS 0702+6043. Bottom: signal in HS 0702+6043 with shifted phases. See text for further descriptions. 

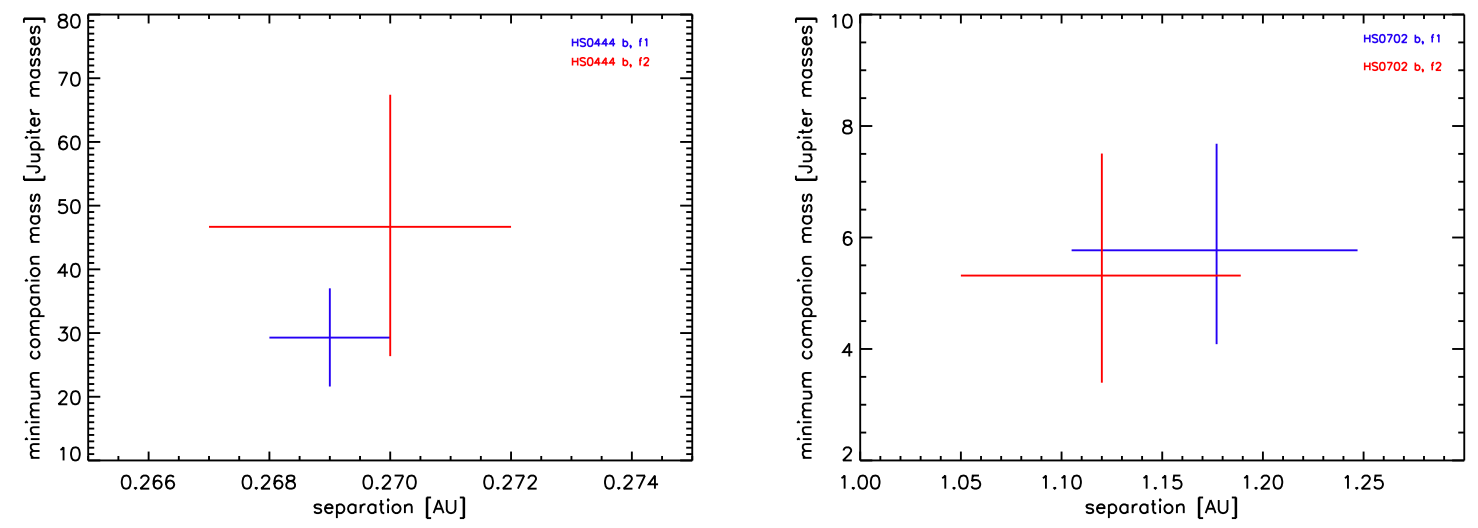

Figure 6.12: Parameter derived for the companion candidates based on f1 (blue) and f2 (red). Left: HS 0444+0458 b. Right: HS 0702+6043 b.

Figures 6.13 and 6.14 show the phase-folded O-C diagrams, respectively. In both cases this is done independently for the two strongest frequencies f1 and f2. The solid lines are given by the orbital characteristics, which are as follows (as in equation 6.1, amplitudes are given in seconds and orbital periods in days):

- $7.502332 \cdot \sin [(2 \pi / 72.001531) \cdot t+43.62546]$ and

- $11.992803 \cdot \sin [(2 \pi / 72.319059) \cdot t+152.35092]$

for HS $0444+0458 \mathrm{~b}$ based on $\mathrm{f} 1$ and f2, respectively, and

- $6.4732662 \cdot \sin [(2 \pi / 659.73265) \cdot t+34.416065]$ and

- $5.6768441 \cdot \sin [(2 \pi / 612.58636) \cdot t+107.16649]$

for HS $0702+6043 \mathrm{~b}$ based on $\mathrm{f} 1$ and $\mathrm{f} 2$, respectively.

Let us now calculate the characteristics of the companion candidates based on the fitted parameters. Using equations 5.29 and 5.31 we are now able to calculate the orbital separations and minimum masses of our companion candidates HS $0444+0458 \mathrm{~b}$ and HS $0702+6043 \mathrm{~b}$. The results are plotted in Figure 6.12. With reference to the corresponding minimum masses, we can conclude that HS $0444+0458$ b is most probably a brown dwarf candidate and HS $0702+6043$ b a planetary candidate. 

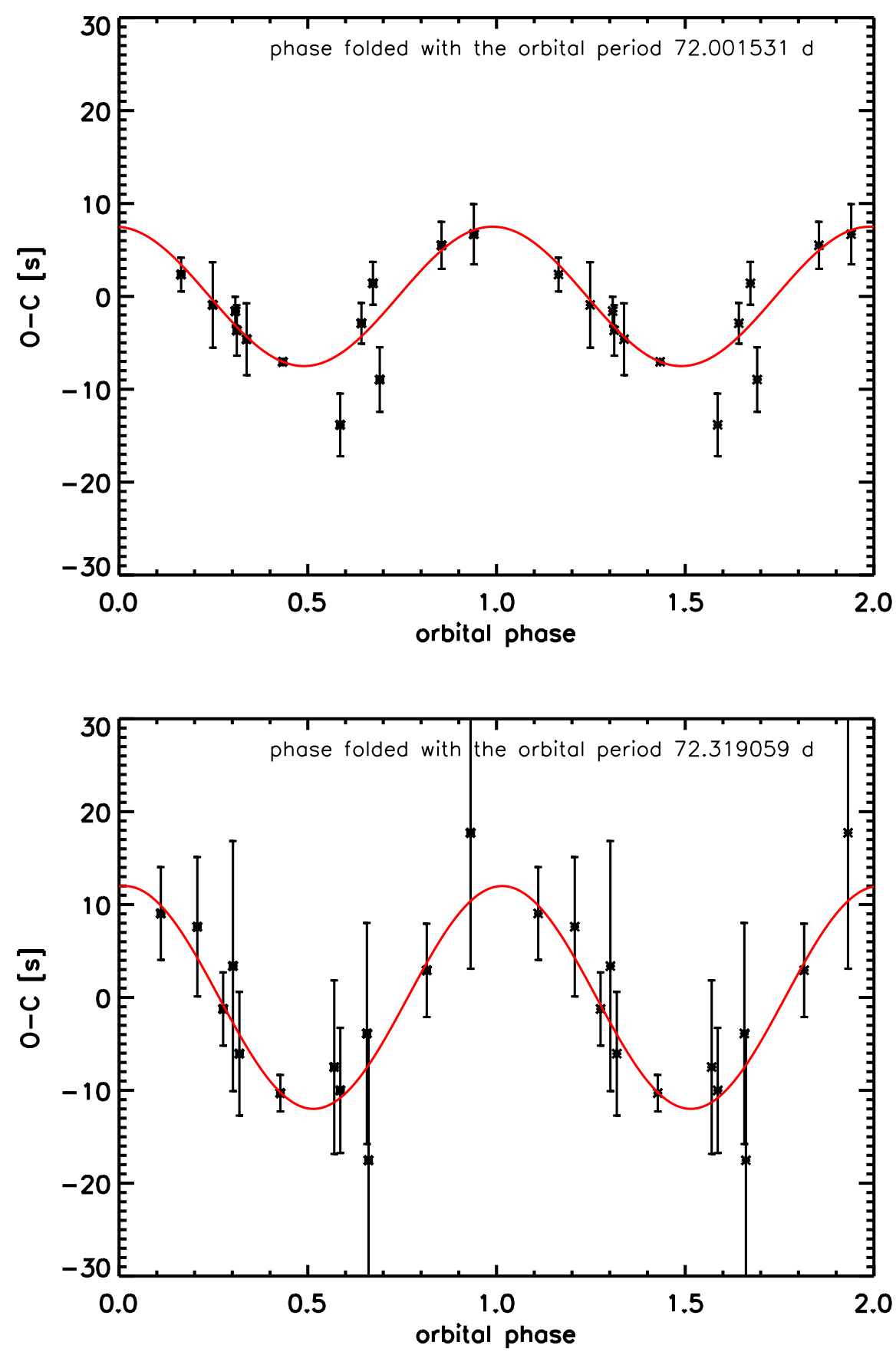

Figure 6.13: Phase-folded $\mathrm{O}-\mathrm{C}$ diagrams for the companion candidate HS $0444+0458 \mathrm{~b}$ based on f1 (top) and f2 (bottom). The points are folded with the orbital periods as given in Table 6.2 (also given in the plots). Data points are duplicated on the phase-axis for visibility reasons. 

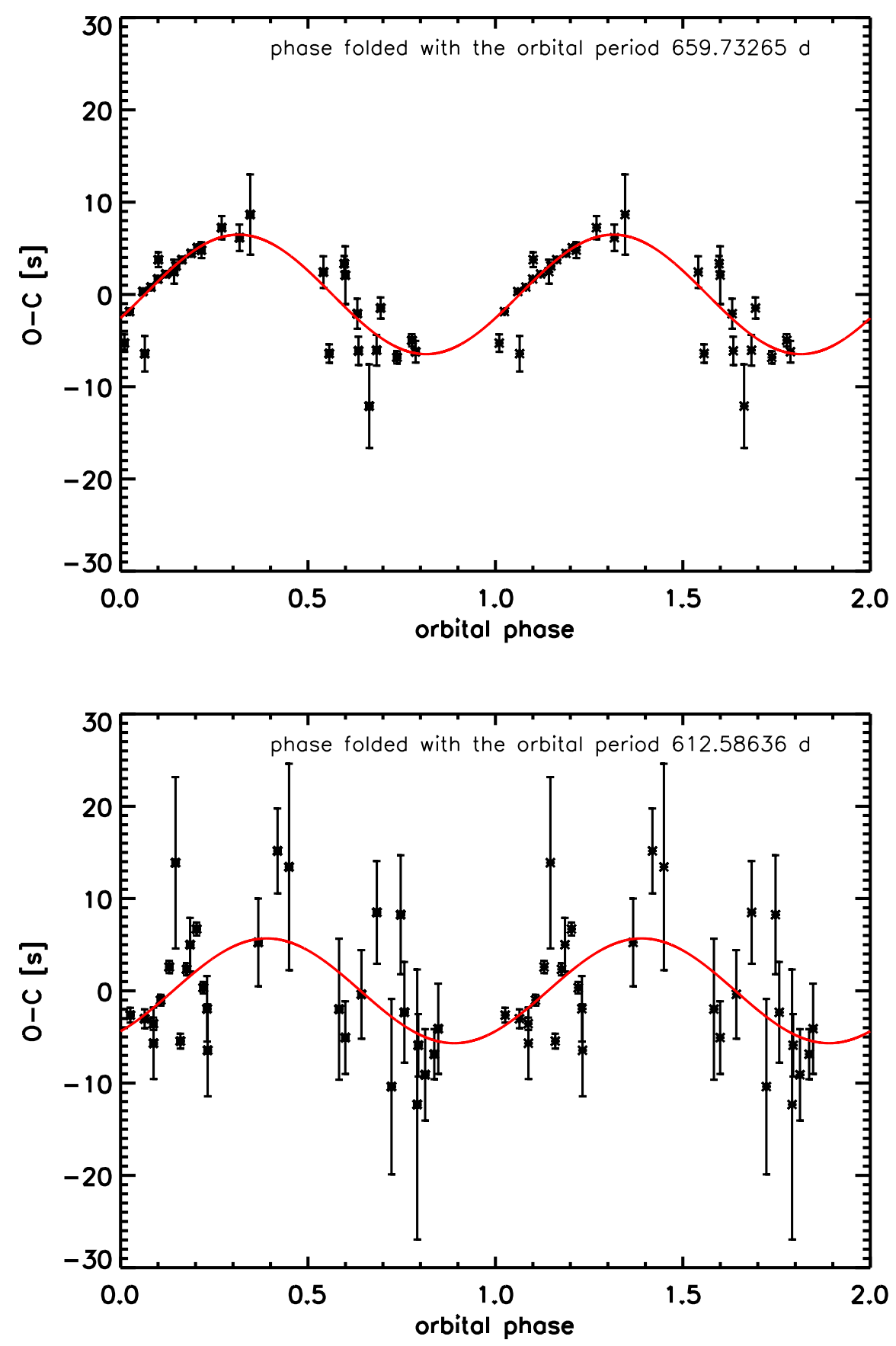

Figure 6.14: Phase-folded $\mathrm{O}-\mathrm{C}$ diagrams for the companion candidate HS 0702+6043 b based on f1 (top) and f2 (bottom). The points are folded with the orbital periods as given in Table 6.2 (also given in the plots). Data points are duplicated on the phase-axis for visibility reasons. 
Table 6.1: Fitting results: $\dot{P}$ and evolutionary timescales (quadratic components) and beating signals (sinusoidal component). The expansion/contraction timescale $R_{\text {evol }}$ will be specified further in section 7.3.2. The reduced $\chi^{2}$ values are the ones including all components, also the orbital signatures given in Table 6.2.

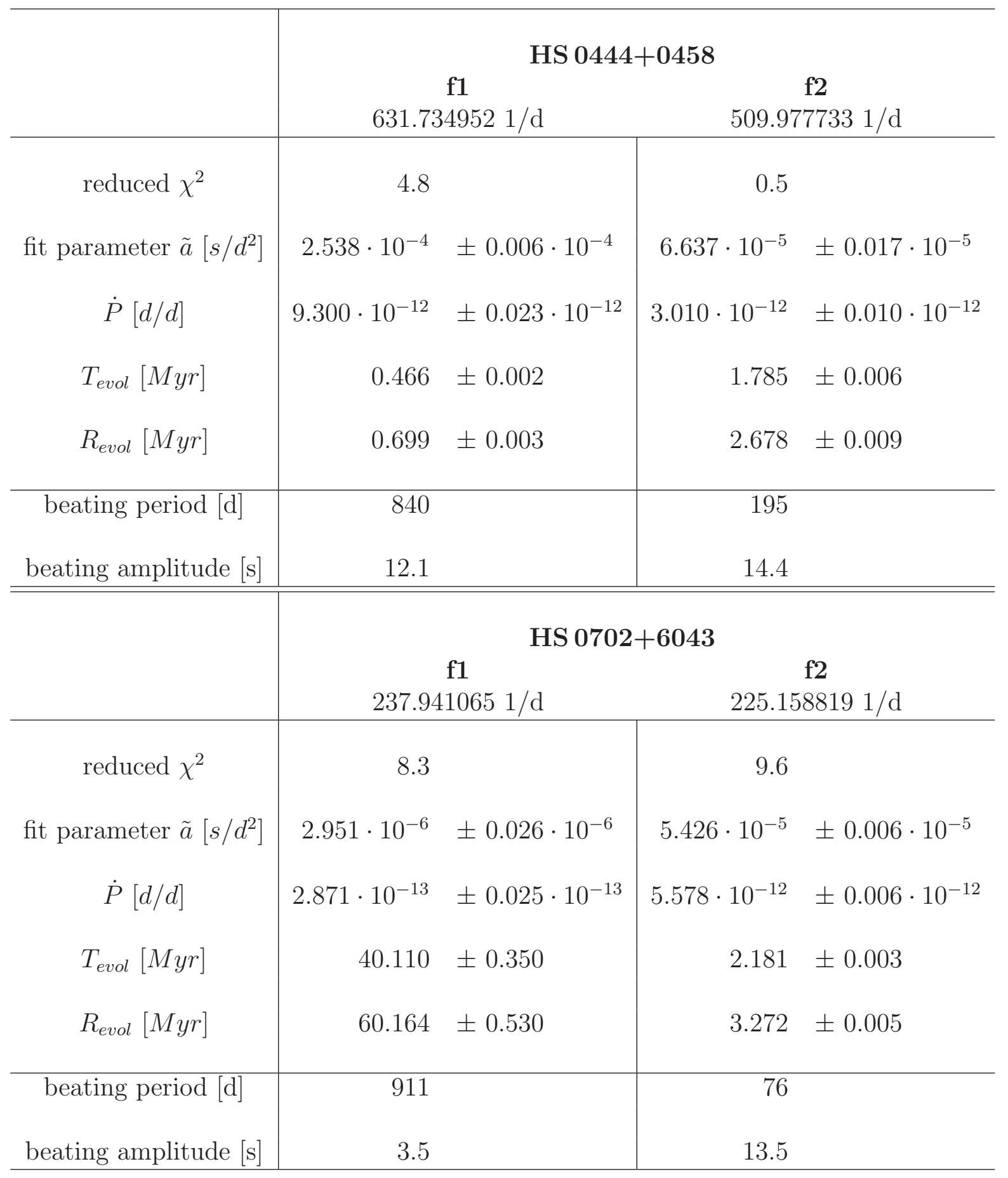


Table 6.2: Fitting results: possible orbital signatures (sinusoidal components). The reduced $\chi^{2}$ values of the full fits (including evolutionary, beating and orbital signatures) are given in Table 6.1. HS $0444+0458 \mathrm{~b}$ is the brown dwarf candidate and HS $0702+6043 \mathrm{~b}$ is the planetary candidate.

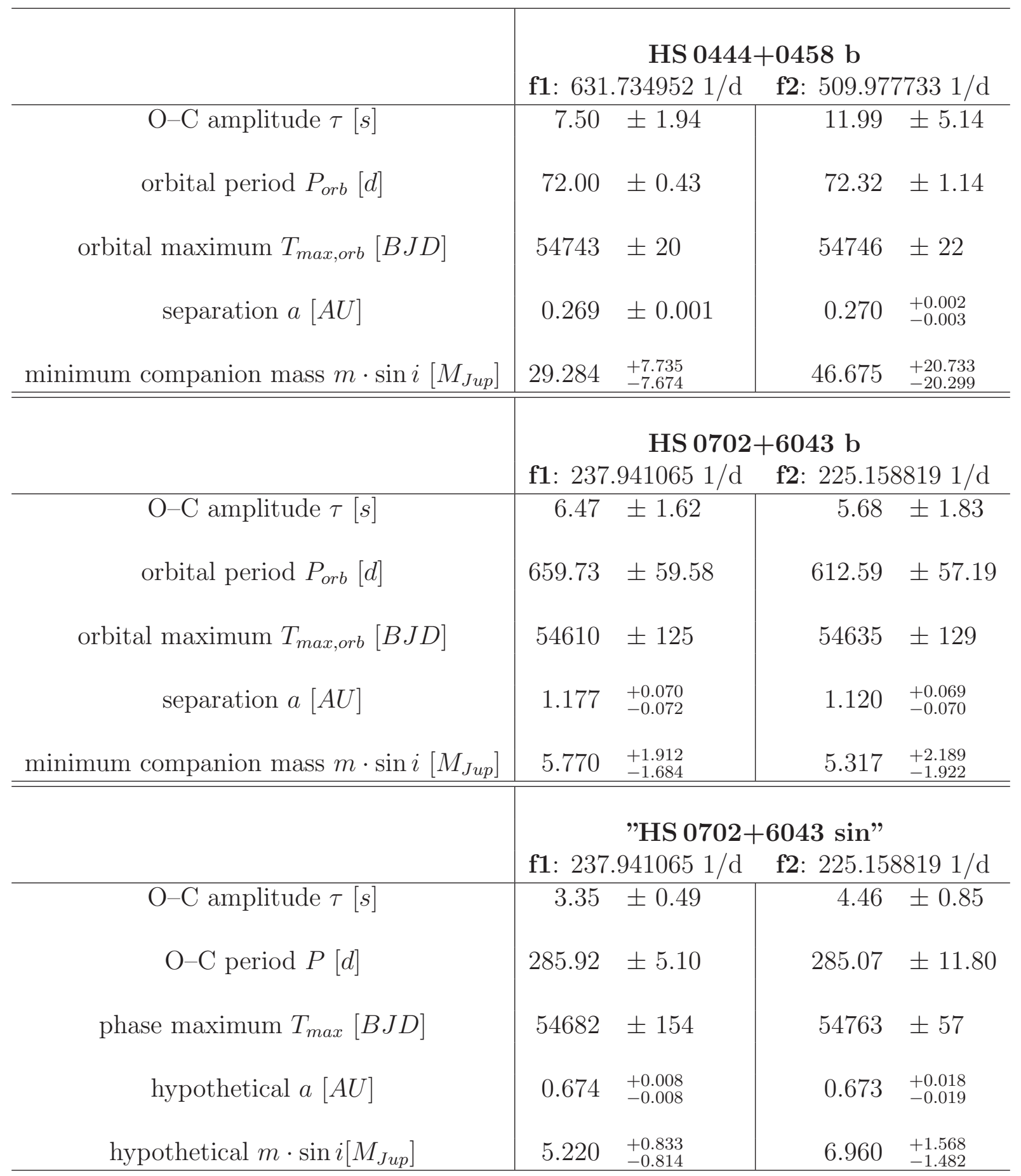



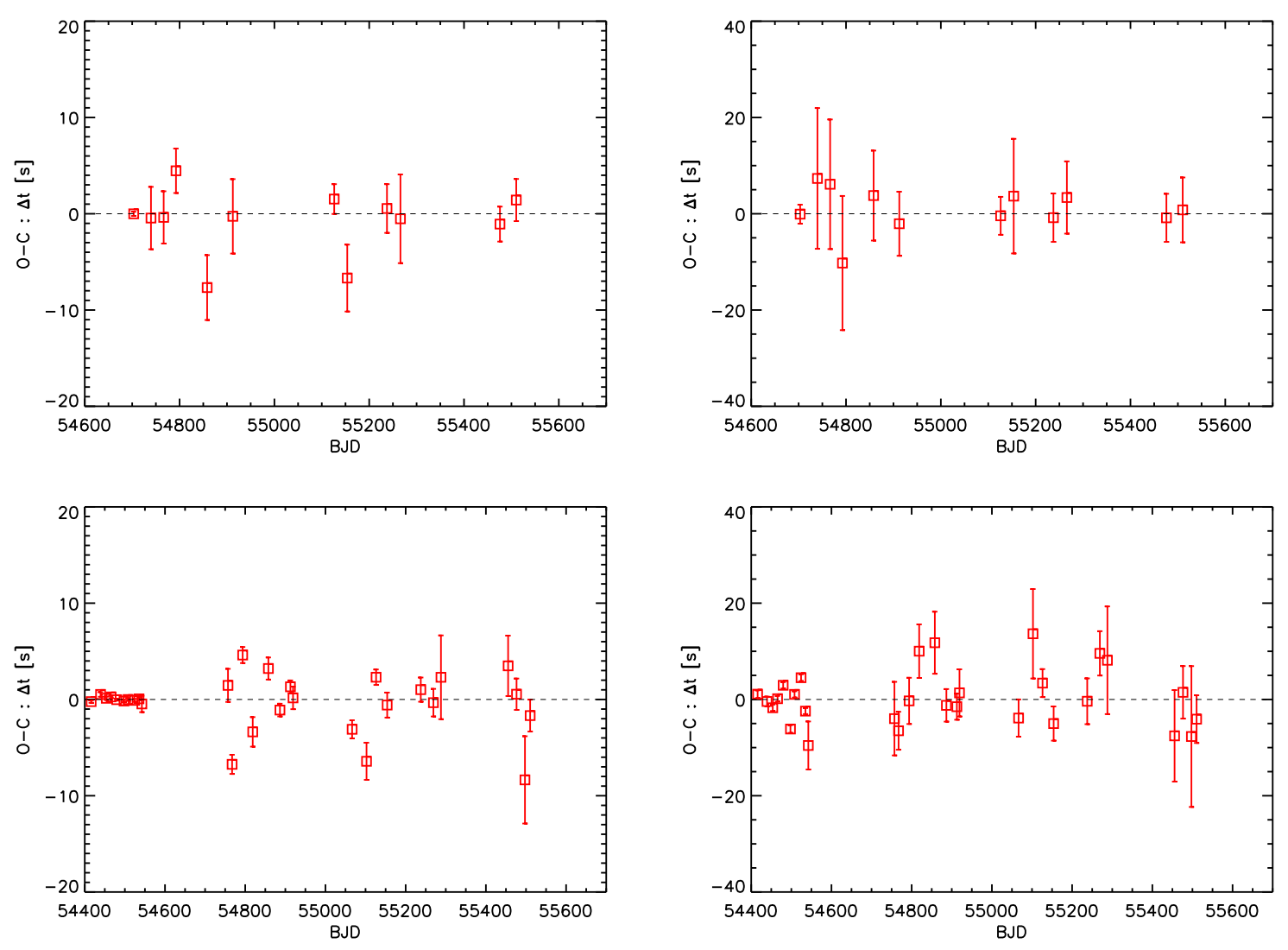

Figure 6.15: O-C residuals. Evolutionary-, beating- and orbital signals are subtracted. Panels are f1 (top left) and f2 (top right) of HS 0444+0458 and f1 (bottom left) and f2 (bottom right) of HS 0702+6043, respectively.

\subsection{4 $\mathrm{O}-\mathrm{C}$ residuals}

The $\mathrm{O}-\mathrm{C}$ residuals after subtraction of all signatures discussed above (i.e. after subtraction of evolutionary-, beating- and orbital components) are presented in Figure 6.15. There are no periodic signatures or systematic trends left in the residuals. To briefly summarize it, in each target we first subtracted the parabolic evolutionary signature, then the sinusoidal beating signature and finally the sinusoidal orbital component(s).

Tabular summaries of all fitted components in the $\mathrm{O}-\mathrm{C}$ diagrams of both our targets are provided in Table 6.1 and Table 6.2. HS $0444+0458 \mathrm{~b}$ and HS $0702+6043 \mathrm{~b}$ in Table 6.2 are the companion candidates whereas "HS $0702+6043$ sin" is the sinusoidal signature of unknown origin. This feature will be further addressed in the discussion. The value $T_{\text {max }, \text { orb }}$ in Table 6.2 marks the time of the first orbital maximum which falls into the 
time range of our data (cf. Figure 6.11 for the orbits and times of orbital maxima).

\subsection{Detection limits for our data sets}

Since the sensitivities of the timing method have previously been calculated more generally, I will now apply them to the characteristics of our particular data set. Figure 6.16 defines the parameter space on the mass vs. separation plane to which we are sensitive with our data sets for HS 0444+0458 and HS 0702+6043, respectively. Thus, we are sensitive to companions located between the two solid vertical blue lines and above the solid sloped red lines. The solid sloped lines correspond to those in Figure 5.13 and represent different amplitude sensitivities in the $\mathrm{O}-\mathrm{C}$ diagrams. Looking at the typical sizes of the error bars of our $\mathrm{O}-\mathrm{C}$ points, we assume the $3 \mathrm{~s}$ limit to be a realistic estimate.

Positions of the vertical blue lines in Figure 6.16 are determined by the total time base of our data sets. The maximum detectable period corresponds to the total length of the data set. In our case this is 28 months for HS 0444+0458 (time span August 2008 - November 2010) and 37 months for HS 0702+6043 (time span November 2007 - November 2010). This maximum orbital period can be transferred to a maximum orbital separation and is represented by the rightmost vertical blue line. Accordingly, the leftmost vertical line represents the shortest detectable separation. At smaller separations, the induced reflex motion of a substellar companion becomes too small to be detected with the timing method.

Dashed horizontal lines separate the different mass regimes of stars, brown dwarfs and exoplanets and the symbols mark the positions of our candidates (see Figure caption).

\subsection{Current status for the other EXOTIME targets}

This thesis focuses on the analysis of the EXOTIME targets HS 0444+0458 and HS $0702+6043$. For the sake of completeness I want to briefly mention the current status for the remaining three EXOTIME targets. As discussed with R. Silvotti (priv.comm.), only the most important issues concerning 

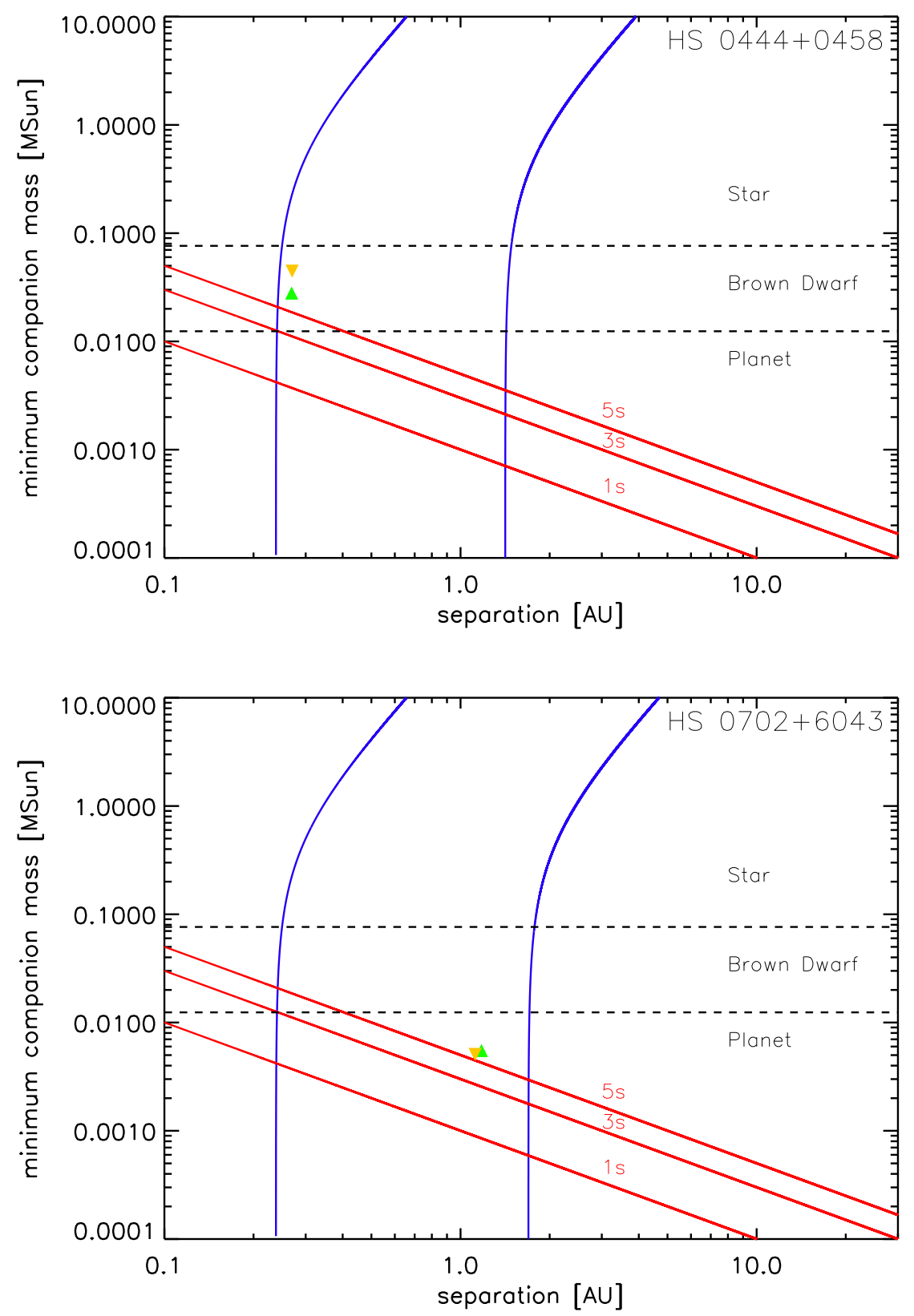

Figure 6.16: Detection limits for our data. Top: HS $0444+0458$. The green triangle is the brown dwarf candidate position derived from $\mathrm{f} 1$ and the orange upside down triangle is the one derived from f2. Bottom: HS 0702+6043. The green triangle is the exoplanet candidate position derived from f1 and the orange upside down triangle is the one derived from f2. Increasing the total time base of the corresponding data sets will shift the rightmost blue (vertical) line towards larger separations and the red sloped lines towards lower companion masses. See text for further details. 
the latter are addressed here but various references for further reading are provided as well.

\subsubsection{HS $2201+2610$}

The detection of a planetary companion to HS 2201+2610 (or V $391 \mathrm{Peg}$ ) was the reason to start the EXOTIME program. All relevant information for this target up to the year 2006 is given in Silvotti et al. (2007). A regular monitoring of this target has been continued after the detection of the companion and is still being conducted in order to improve the orbital solution. The incorporation and addition of new $\mathrm{O}-\mathrm{C}$ points to the existing solution is subject of an on-going analysis, conducted by R. Silvotti.

The true mass of the planet V $391 \mathrm{Peg}$ b is still unknown because of the unknown inclination. To determine the inclination of this system is therefore of main priority. The basic idea is as follows: as a first step one could detect and measure the rotational splitting in the Fourier transform. This would deliver the rotational velocity $v_{\text {rot }}$. In a second step one could measure the broadening of spectral lines and deconvolve the pulsational broadening from the rotational broadening. This would give the projected rotational velocity $v_{\text {rot }} \cdot \operatorname{sini}$. If the pulsational and rotational axes are assumed to be aligned, one could then derive the stellar inclination. If the stellar inclination is a primer for the orbital inclination, one could then calculate the true mass. Attempts to derive the inclination of HS $2201+2610$ and therefore the true mass of the planet are presented in Schuh et al. (2009) and Kruspe et al. (2008). So far, there are indications that HS $2201+2610$ is probably a slow rotator and that pulsational radial velocity amplitudes are very small.

Multicolor photometry has the potential to be used for mode identification (Randall et al., 2005) and recently a robust mode identification for this target was obtained from multicolor WHT/ULTRACAM photometry for the two main pulsation frequencies: f1 appears to be $l=0$ while $\mathrm{f} 2$ is identified as $l=1$ (Silvotti et al., 2010). This implies that $\mathrm{f} 2$ should be split by the stellar rotation into 3 close frequencies which, however, are not detected in the same data because of poor resolution and/or very low amplitudes (Silvotti, private comm.). 


\subsubsection{PG $1325+101$}

Although the data amount available for PG 1325+101 at the present time is not enough to perform a full and robust $\mathrm{O}-\mathrm{C}$ analysis, it is nevertheless possible to ensure the short-term phase stability of the main pulsation period at 137.8 s. Such an analysis, based on EXOTIME data from January 2009 to May 2009 and strengthened by another campaign in early 2008, is provided in Benatti et al. (2010).

Encouraged by the preliminary analysis of PG $1325+101$ and also by the results found for the two targets analyzed in the present thesis, PG 1325+101 is now the highest priority EXOTIME target.

Multi-site observations (Silvotti et al., 2006) revealed a dominant pulsation period at $137.8 \mathrm{~s}$ and several $(>15)$ other periods with lower amplitudes. Asteroseismic modeling (Charpinet et al., 2006) best explains the dominant mode in terms of $l=2, k=2$, even though close frequencies due to the rotational splitting are not clearly seen, maybe because of a low inclination. From time resolved spectroscopy Telting \& Østensen (2004) could exclude a close companion and suggested $l=0$ for the dominant pulsation mode. Following Baran et al. (2010) only $l>2$ can be excluded for the $137.8 \mathrm{~s}$ period, although $l=0$ remains the most likely option.

\subsubsection{EC 09582-1137}

The EXOTIME data archive for EC 09582-1137 is very poor (see Table 4.13). This is mainly due to the low declination of the target and the low amplitudes of the oscillations. This results in short observability throughout the year with the telescopes available to EXOTIME as well as a restriction to the facilities with larger mirror-sizes $(>2 \mathrm{~m})$. With the current rate of observations it will probably take a few more years to achieve first reliable $\mathrm{O}$ C diagrams. Apart from the EXOTIME program, Kilkennv (2010) examined amplitude variations in EC 09582-1137 and Randall et al. (2009) present a detailed asteroseismological analysis. 


\section{Chapter 7}

\section{Discussion}

In this chapter I want to discuss the previous results, put them into a broader context and present some applications.

Beginning with a further characterization of the candidates, I will provide estimations of true masses, potential radial velocity signals and effective temperatures. Subsequently, aspects of $\mathrm{sdB}$ formation will be addressed and in particular the outcome of a common envelope evolution is considered. Finally I will expand on the evolutionary status of our targets themselves and the relation of these evolutionary aspects to asteroseismic models.

\subsection{Alternative interpretations and possible misinter- pretations}

More than 30 years ago Percy et al. (1980) stated:

'The study and interpretation of period changes in variable stars has had a long and sometimes dubious history. In a few cases, it has produced interesting and worthwhile results. In most cases, however, it is a source of confusion and frustration. The value of such work should be carefully considered before it is undertaken. ${ }^{1}$

I want to assure the reader that the last sentence of this statement has carefully been considered, but in order to avoid the confusion and frustration mentioned in the above statement, I want to emphasize some important aspects which have to be kept in mind during the following discussions.

\footnotetext{
${ }^{1}$ Literally adopted from Percy et al. (1980).
} 
- For the calculation of the evolutionary timescales we assume the evolution of the sdB stars to be linear, i.e. the evolution of the star leads to a linearly and monotonically changing period, represented in the $\mathrm{O}-\mathrm{C}$ by a parabolic shape. Sudden non-linearities in the period evolution of sdB stars are not expected to be a dominating effect and there is a strong support that our assumption of a linear, monotonic period evolution is valid to first order (see Charpinet et al., 2002b, for further details). I will expand on different phases of sdB evolution and their influence on the secular period changes later in this chapter when the evolutionary status of the host star is considered.

- Proper motion of the stars will also lead to a linear change in the period. In the last chapter we calculated its influence to be negligible compared to the evolutionary contribution.

- Sinusoidal signatures in $\mathrm{O}-\mathrm{C}$ diagrams are often interpreted with the presence of a companion. However, we have seen that beating will also cause sinusoidal shapes. The inspection of amplitude- and phase variability is a way to investigate whether a sinusoidal component is due to a beating effect. Once beating can be excluded as origin of a sinusoidal $\mathrm{O}-\mathrm{C}$, the companion hypothesis remains as the most likely explanation. However, this has not to be always true. A study of one pulsation period in the DAV white dwarf G 29-38 by Winget et al. (1990) showed that the phase of this pulsation changed but no corresponding change in the amplitude was found. This excluded an intrinsic cause of the phase variation since beating or nonlinear interactions like mode coupling (Buchler et al., 1995, 1997) all go along with amplitude modulation. Hence, Winget et al. (1990) interpreted the phase variation with a companion as extrinsic cause, which fitted the data nicely. However, neither the predicted RV signal nor a consistent phase variation of another pulsation mode could be detected in follow-up observations, which disproved the companion interpretation.

This shows the need of conducting the $\mathrm{O}-\mathrm{C}$ analysis with at least two independent modes as a consistency check.

More recently, Dalessio et al. (2011) demonstrated that it is indeed highly desirable to conduct the $\mathrm{O}-\mathrm{C}$ analysis not only for one single 
mode, but independently for more than one frequency. They analyzed four modes in the pulsating white dwarf EC 20058-5234 with the following results. In addition to parabolic $\mathrm{O}-\mathrm{C}$ contributions, the authors detect a sinusoidal component of the same period in all four modes. However, the amplitudes of the phase variations are different and two of the variations have a relative phase of zero and the other two a relative phase of $\pi$. In conclusion, the sinusoidal behavior in the $\mathrm{O}-\mathrm{C}$ diagram of EC 20058-5234 cannot be attributed to a companion since it is not supported by any of the other modes. Some yet unknown effect must be responsible for these appearances in the $\mathrm{O}-\mathrm{C}$ diagrams of EC 20058-5234. Another interesting aspect is that two of the modes considered have a positive $\dot{P}$ and two have a negative one.

These two examples, as well as the results of this thesis, show that a sinusoidal variation in one single mode alone is inconclusive and I want to emphasize that a companion origin should only be claimed if the same sinusoidal variation of a mode (i.e. same amplitude, period and orbital phase) can be verified by at least one other mode independently and consistently.

\subsubsection{Treatment of the beating}

For our targets, the observed amplitude variability in correlation with the observed phase modulation ${ }^{2}$ could very well be explained by beating of closely spaced frequencies, which was intensively discussed and justified in the analysis.

Let us now briefly comment on the influence of the beating signatures on the measured $\dot{P}$ values. The presence of two close components should imply the removal of two quadratic $\mathrm{O}-\mathrm{C}$ components but the subtraction of only one quadratic component is nevertheless valid since the sum of two parabolae is still a parabola, but then the measured $\dot{P}$ should be regarded as a weighted average of the two components.

One possibility to explain a doublet signature as close to each other as in

\footnotetext{
${ }^{2}$ The theoretically expected phase modulation in the case of beating of closely spaced frequencies is not strictly sinusoidal. The most extreme case appears if the amplitudes are exactly equal, which results in a half cycle phase jump at every new cycle of the beat period. However, as soon as the amplitudes differ, this phase jump is progressively smeared out and the phase modulation adopts a quasi-sinusoidal shape. See Breger \& Bischof (2002) or Breger (1981) for details.
} 
our cases might be incorrect pre-whitening. If a slightly wrong frequency was used to subtract the strongest frequency, then another peak very close to the previous one would appear in the periodogram and mimic a close doublet, which belongs to the same mode. We are not successful in finding a slightly different value for the strongest peak that would be able to avoid the substructure. Hence, a pre-whitening error is unlikely. Even in the unlikely case that this would have happened to be the origin for the beatings in HS 0444+0458 and HS 0702+6043, the removal of the beat signature should have no effect on the measured $\dot{P}$ value, since both peaks subject to the beating would belong to the same physical mode.

Another possible explanation for the close frequency pairs could be rotational splitting in a situation where, e. g. for a triplet with unequal amplitudes, only two components are above the noise and observationally detectable. The different $m$ components of a rotationally split mode should have very similar $\dot{P}$ values, because they belong to the same pulsation mode. The measured average would in that case not differ much from the $\dot{P}$ values of the individual split components. Although rotational splitting could possibly cause similar beat patterns in independent modes, these would probably only be of same period and/or amplitude but not of same relative phase since the phases of independent frequencies should be randomly different from one frequency to another. Hence there should be no risk that rotational splittings could be misinterpreted with an orbital origin.

The treatment of the beating has to be considered for $\mathrm{f} 1$ and $\mathrm{f} 2$ in both targets separately. Neither of those two frequencies has an influence on the beating signature of the respective other one. Hence, the treatment of the beating has no effect on our interpretation of those signatures which we explain with an orbital origin because there the same period, amplitude and relative phase are seen in $\mathrm{f} 1$ and $\mathrm{f} 2$ independently.

\subsubsection{The HS 0702+6043 "sin" signal}

As explained in the analysis, the signal HS 0702+6043 "sin" cannot be of extrinsic, i. e. orbital origin, since the phasing is inconsistent in the two independent modes f1 and f2. Also a beating origin is unlikely because beating shows a shift of $\pi / 2$ between the amplitude- and phase variation of 
the same (unresolved) frequency, but here the shift of $\pi / 2$ is between the relative phase variations of two well separated frequencies. Furthermore, the two frequencies $\mathrm{f} 1$ and $\mathrm{f} 2$ are not close. Since only modes with close frequencies are subject to beating, this is hence not expected here at all! The present phenomenon seems to be related to the situation in the white dwarf EC 20058-5234 (Dalessio et al., 2011), but with the difference that in the latter case the relative phasings are shifted by $\pi$, instead of $\pi / 2$ as in our case.

The cause of these phenomena is yet unknown and needs to be investigated further.

\subsubsection{Assumption of circular orbits}

In the analysis we restricted the orbits of our companion candidates to be circular, i. e. strictly sinusoidal signatures in the $\mathrm{O}-\mathrm{C}$ diagrams. This is justified by looking at the $\mathrm{O}-\mathrm{C}$ points, which indeed do not show a significant deviation of a sinusoidal shape. Hence, the eccentricity should be small. With the similar argument based on the sinusoidal shape of the $\mathrm{O}-\mathrm{C}$, the same conclusion was also drawn for HS $2201+2610$ by Silvotti et al. (2007), who state that the eccentricity is close to zero.

In addition we also incorporated the eccentricity as another free parameter during the fitting with MPFIT. We found no significant improvement by including the eccentricity to the fits and since we wanted to keep the number of free parameters as low as possible (owing to the small number of $\mathrm{O}-\mathrm{C}$ data points), we assumed the eccentricity to be zero.

With the arguments given above, this assumption is valid and justified.

\subsection{Characteristics of the companion candidates}

\subsubsection{Overview of companion candidates}

After removal of the beating signatures we detect three sinusoidal variations in the O-C diagrams of our targets HS $0444+0458$ and HS $0702+6043$ which we had termed HS 0444+0458 b, HS 0702+6043 b and "HS 0702+6043 sin" in the previous chapter. Keeping in mind the aspects mentioned above, we claim the following of these $\mathrm{O}-\mathrm{C}$ signatures to suffice for the status of com- 
panion candidates: HS $0444+0458 \mathrm{~b}$ and HS $0702+6043 \mathrm{~b}$.

We justify this statement as follows:

- HS $0444+0458$ b: this sinusoidal variation is, within the error bars, detected with the same period and amplitude in two modes independently. More importantly, the orbital position of the hypothetical companion is the same in both frequencies considered (cf. Figures 6.11 and 6.13). We therefore have a strong support that this sinusoidal variation is indeed due to a companion. Based on the measured parameters (separation, minimum mass) we claim HS $0444+0458$ b to be a brown dwarf candidate.

- HS 0702+6043 b: the same argumentation as for HS $0444+0458$ b is applied for this case. Based on the measured parameters (separation, minimum mass) we claim HS $0702+6043$ b to be a exoplanet candidate.

- "HS 0702+6043 sin": we measure a sinusoidal variation of same amplitude and period in two independent modes. However, the phases are shifted roughly by $\pi / 2$ meaning that a hypothetical orbital position is not consistent in these two modes considered. The signature "HS 0702+6043 sin" is therefore no companion candidate and its origin has to be investigated further.

\subsubsection{Constraints on companion masses}

Due to the unknown orbital inclination, all masses derived for possible companions with the timing method are only minimum masses (see Figure 5.7 and Equation 5.29).

Assuming a random distribution of orbital inclinations $i$, the probability $\mathcal{P}$ for an orbital inclination $i$ to be lower than a certain value $\theta$ can be estimated as $\mathcal{P}(i<\theta)=1-\cos \theta$. For the substellar companion candidates around our two main targets HS $0444+0458$ and HS 0702+6043 we can calculate which inclination value would be necessary to put their true masses towards the separation boundary between the (exoplanet / brown dwarf) and (brown dwarf / star) regimes, respectively. These estimations are summarized in Table 7.1 and visualized in Figure 7.1 . 

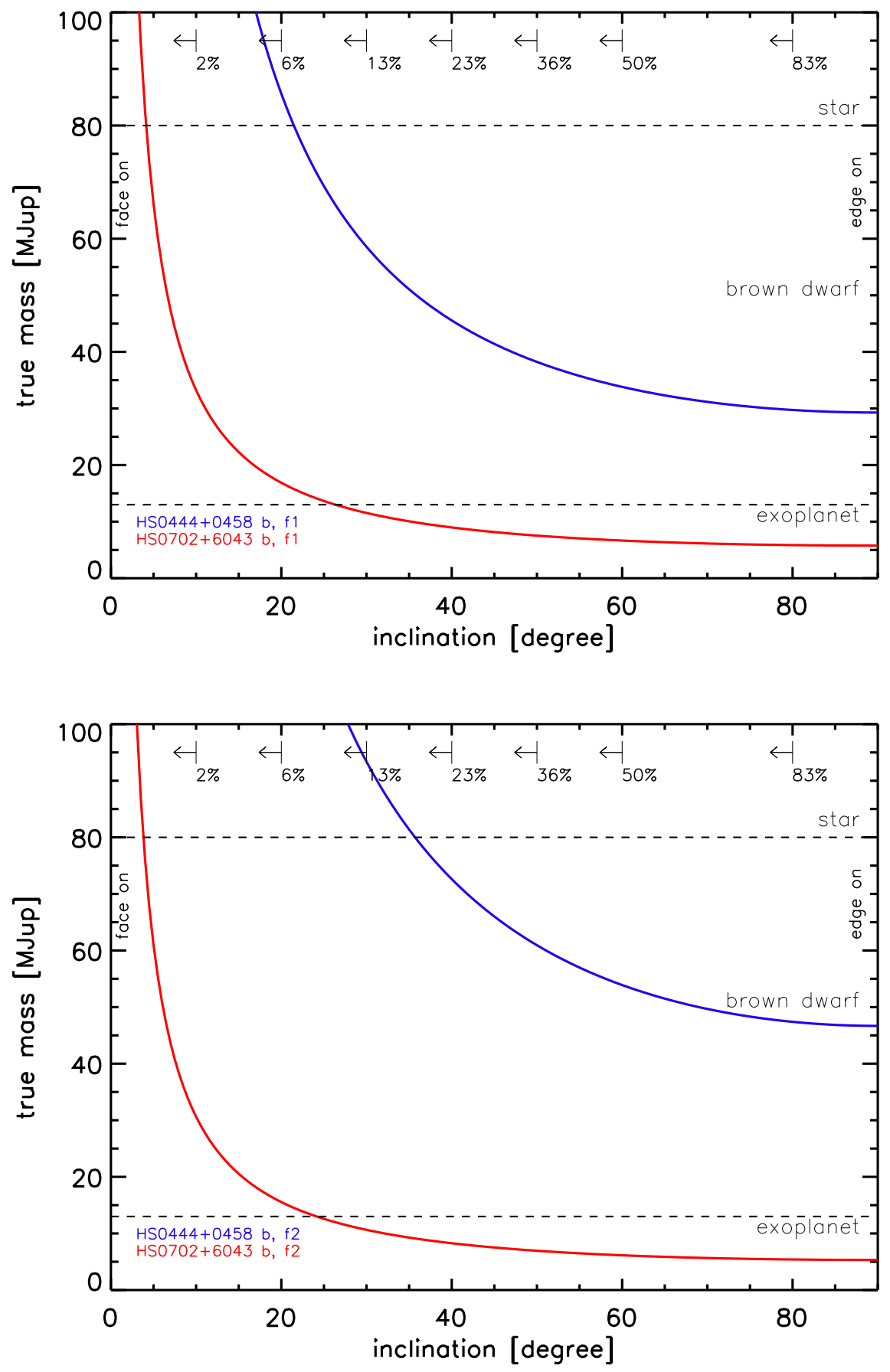

Figure 7.1: True masses as a function of the unknown inclination for the sinusoidal signatures according to f1 (top) and f2 (bottom). The dashed lines separate the stellar, brown dwarf and exoplanetary regimes. The arrows mark the probabilities that the inclination is smaller than a certain value (see text for details). 
If the orbital inclinations would be at such a low value that the true masses of the above mentioned candidates would place them to the stellar regime, the nature of such a low-mass stellar companion to a subdwarf B star would most probably be a late-type main-sequence star or a white dwarf. This is suggested by recent studies on the nature of the secondaries in close subdwarf B binaries (see chapter 6 in Heber, 2009). The shape of the light curve is a principle diagnostic tool to discriminate between the two types of low-mass stellar companions in close systems: a main sequence companion might be detected via its reflection effect in the light curve. The reflected light of the hemisphere facing the sdB primary will vary with the orbital period. A white dwarf companion would not show such a reflection effect since the irradiated area is too small. However, it will produce a signature in the light curve due to tidal forces. The tidal forces of the compact white dwarf companion will deform the spherical shape of the sdB and cause a so-called ellipsoidal variation: due to the elongated sdB shape, two maxima and two minima will occur in the light curve during one full orbital period and hence cause a peak at half the orbital period in a periodogram.

Mazeh \& Faigler (2010) estimate the "order-of-magnitude" effects of reflection effects and ellipsoidal variations on the light curves. The semiamplitude of an ellipsoidal variation is roughly estimated to be $A_{\text {ellip }} \approx$ $m_{\text {comp }} / M_{s d B} \cdot\left(R_{s d B} / a\right)^{3}$ and the semi-amplitude of the reflection effect to be roughly $A_{\text {refl }} \approx \rho_{\text {geo }} \cdot\left(r_{\text {comp }} / a\right)^{2}$ with $\rho_{\text {geo }}$ being the geometrical albedo (see details in Mazeh \& Faigler, 2010). However, if the companion candidates to our targets would be white dwarfs (with a very low probability, see above), the amplitude of an ellipsoidal signature would not be detectable with our

Table 7.1: Effects of the unknown inclinations on the true masses and the type of the companion candidates. $i_{\text {star }}$ is the inclination limit dividing the stellar and brown dwarf regimes (we use $80 M_{J u p}$ ) for our candidates and $i_{B D}$ is the one dividing the brown dwarf and exoplanetary regimes (we use $13 M_{J u p}$ ).

\begin{tabular}{c|cc|cc|cc}
\hline candidate & frequency & minimum mass $\left[M_{\text {Jup }}\right]$ & $i_{\text {star }}$ & $\mathcal{P}_{i, \text { star }}$ & $i_{B D}$ & $\mathcal{P}_{i, B D}$ \\
\hline HS 0444+0458 b & $\mathrm{f} 1$ & 29.284 & 21.47 & $6.9 \%$ & & \\
& $\mathrm{f} 2$ & 46.675 & 35.69 & $18.8 \%$ & & \\
\hline HS 0702+6043 b & $\mathrm{f} 1$ & 5.770 & 4.14 & $0.3 \%$ & 26.35 & $10.4 \%$ \\
& $\mathrm{f} 2$ & 5.317 & 3.81 & $0.2 \%$ & 24.14 & $8.7 \%$ \\
\hline
\end{tabular}


present data. This can easily be verified by inserting our measured parameter in the above estimations which roughly yields $10^{-6} \mathrm{mma}$ and $10^{-9} \mathrm{mma}$ for HS 0444+0458 and HS 0702+6043, respectively.

If the companion candidates would be of main sequence type (again with very low probabilities as shown above), we have already demonstrated in Figure 3.5 that an infrared excess caused by a main sequence companion earlier than M0 can be excluded. Companions later than M0, which would not reveal themselves by an IR-excess would, according to the estimation given above, cause a semi-amplitude reflection effect not detectable with our current data. Even for the case of a "perfect mirror" the estimations above yield reflection effects of roughly $3 \cdot 10^{-3} \mathrm{mma}$ and $4 \cdot 10^{-4} \mathrm{mma}$ for HS $0444+0458$ and HS 0702+6043, respectively, which is clearly below our detection limits. One should also keep in mind that ellipsoidal variations and reflection effects are more easily detectable in very close binary systems. Since our candidates are in rather wide orbits, it is quite obvious that a reflection- or ellipsoidal effect on a timescale of hundred days or more are not detectable in our data.

As a final side-note, let us compare again the J-H colors of those EXOTIME targets which host a substellar companion. HS $0444+0458$ has a J-H of 0.043 which is much higher than the values -0.123 and 0.004 , respectively, for HS $0702+6043$ and HS $2201+2610$. Looking at the companion masses, the brown dwarf around HS 0444+0458 has a much higher mass than the exoplanets around HS 0702+6043 and HS 2201+2610. Hence, this would likely explain the excess of HS $0444+0458$ compared to the other targets. However, this excess is still below the limit of 0.065 which divides stellar from substellar companions in the study of Reed \& Stiening (2004).

Summarizing these aspects of inclination, IR-excess, reflection effect and ellipsoidal variation as sketched above, we can state that the companion candidates to HS 0444+0458 and HS 0702+6043

- are substellar with a very high probability $(>81.2 \%$ and $>99.7 \%$, respectively) or,

- if not substellar, they are either of main sequence type later than M0 or a white dwarf 


\subsubsection{Predicted radial velocity signals}

Since we know the minimum masses of our companion candidates, we can calculate the RV variations induced by these on their host star via the binary mass function

$$
f(M)=\frac{M_{c}^{3} \cdot \sin ^{3} i}{\left(M_{c}+M_{s d B}\right)^{2}}=\frac{P K^{3}}{2 \pi G}
$$

where $M_{c}$ and $M_{s d B}$ are the companion mass and sdB mass (we use the canonical sdB mass of $\left.0.47 M_{\odot}\right) . P, K$ and $G$ are the orbital period, radial velocity semi-amplitude and gravitational constant, respectively. In an edgeon case (inclination of 90 degrees) the RV semi-amplitudes induced on the star by the companion candidates HS 0444+0458 b and HS 0702+6043 b would be $2.27 \mathrm{~km} / \mathrm{s}(3.61 \mathrm{~km} / \mathrm{s})$ for HS $0444+0458$ according to f1 (f2) and $0.21 \mathrm{~km} / \mathrm{s}(0.20 \mathrm{~km} / \mathrm{s})$ for HS $0702+6043$ according to f1 (f2), respectively. As already mentioned in section 5.7, RV measurements for the faint and high gravity sdB targets are not an easy task to do. Even though it seems challenging for HS $0702+6043$, the RV variations in HS 0444+0458 are much larger and an attempt to verify these predicted RV signals should be a high priority task on the 'to do list' of further studies concerning HS 0444+0458. Recent status reports in the progress of building up RV surveys to find substellar companions to sdB stars are given by e.g. O'Toole et al. (2011) and Classen et al. (2011). The former state that previous surveys for RV variability typically had limits of $2-3 \mathrm{~km} / \mathrm{s}$ and that the survey of a sample of bright sdB stars they are currently building up aims to achieve a sensitivity of $1-2 \mathrm{~km} / \mathrm{s}$.

\subsubsection{Prediction of $\mathrm{O}-\mathrm{C}$ points}

With the ephemeris of our current $\mathrm{O}-\mathrm{C}$ solutions we are able to make predictions for further $\mathrm{O}-\mathrm{C}$ points. This is not only true for future data, but also for data in the past. As can be seen in Table 4.3, there are data available from the years 2005 and earlier. These are divided from the beginning of our $\mathrm{O}-\mathrm{C}$ analysis by almost three years. In order to include e. g. the data from January 2005 to the current O-C solution, one has to assure that an inclusion of those data would not produce a cycle count error. In this context, Mullally (2007) gives an estimation on the maximum length that is 
allowed between two observations to avoid a cycle count error. According to equation (4.4) in Mullally (2007) this maximum length $t$ of a gap between observations may be estimated as $t=\left[P_{0}\left(P_{0}-\Delta P\right)\right] / \Delta P$ with $P_{0}$ and $\Delta P$ being the measured period and the period uncertainty, respectively. If we assume the frequency resolution to be approximated with the inverse of the total time base of our HS 0702+6043 data set comprising the $\mathrm{O}-\mathrm{C}$ diagrams (i.e. Nov 2007 to Nov 2010) and insert the value for the strongest pulsation f1 in HS 0702+6043, we find the maximal observation gap to avoid a cycle count error to be roughly 1100 days. This is now just enough to be able to include the data from January 2005. The goal of a further study should therefore be to check the current $\mathrm{O}-\mathrm{C}$ solution by deriving and including the corresponding $\mathrm{O}-\mathrm{C}$ point of January 2005.

\subsubsection{Effective temperatures of the companion candidates}

As it has been done for HS $2201+2610 \mathrm{~b}$ in the supplementary information of Silvotti et al. (2007), we now want to roughly estimate the effective temperatures of our candidates.

Let $F_{\text {in }}$ be the flux absorbed by the companion. One has

$$
F_{i n}=\frac{\sigma T_{\mathrm{eff}, \mathrm{s}}^{4} \cdot 4 \pi r_{s}^{2}}{4 \pi a^{2}} \cdot(1-A) \pi r_{p}^{2}=\frac{L_{s}}{4 \pi a^{2}} \cdot(1-A) \pi r_{p}^{2}
$$

with $\sigma$ being the Stefan-Boltzmann constant and $A$ being the Bond albedo of the companion. Here the subscripts $s$ and $p$ stand for star and planet (brown dwarf), respectively. The flux $F_{\text {out }}$ re-radiated by the companion can be expressed as

$$
F_{\text {out }}=\beta \cdot 4 \pi r_{p}^{2} \sigma T_{\mathrm{eq}, \mathrm{p}}^{4}
$$

with $T_{\text {eq,p }}$ being the companion equilibrium temperature (i.e. the temperature it would have in the absence of internal heat sources) and $\beta$ being fraction of the surface that re-radiates. $\beta$ can reach values between 0.5 and 1 . In the case of tidally locked rotation, $\beta$ would be 0.5 since only the dayside would re-radiate (if no atmosphere is present). If, however, the whole surface takes part in the re-radiation, then $\beta=1$, which we assume to be the case in the following. We can now estimate the companion equilibrium 
temperature by balancing $F_{\text {in }}$ and $F_{\text {out }}$ :

$$
\frac{L_{s}}{4 \pi a^{2}} \cdot(1-A) \pi r_{p}^{2}=4 \pi r_{p}^{2} \sigma T_{\mathrm{eq}, \mathrm{p}}^{4}
$$

and therefore

$$
T_{\text {eq,p }}=\left(\frac{L_{s}}{16 \sigma \pi a^{2}}(1-A)\right)^{1 / 4}
$$

Assuming a Jupiter-like bond albedo of $\mathrm{A}=0.343$ we calculate the following equilibrium temperatures:

- HS $0444+0458$ b: $T_{\text {eq }}=1216_{+184}^{-158} K$

- HS $0702+6043$ b: $T_{\text {eq }}=572_{+61}^{-57} K$

The effective temperature is given by $\sigma T_{\text {eff }}^{4}=\sigma T_{\text {eq }}^{4}+\sigma T_{\text {int }}^{4}$, with $\sigma T_{\text {int }}^{4}$ being the internal heat flux of the companion. If we assume the companion internal flux to be negligible compared to the incoming sdB flux we may then approximate $\sigma T_{\text {eff }}^{4} \approx \sigma T_{\text {eq }}^{4}$ and therefore the effective temperatures

- HS $0444+0458$ b: $T_{\text {eff }} \approx 1216_{+184}^{-158} K$

- HS $0702+6043$ b: $T_{\text {eff }} \approx 572_{+61}^{-57} K$

The albedo has a significant effect on the temperature estimations but since we do not know it for our companion candidates, we used the one known for Jupiter. Hence, the above estimations should be treated with caution. The induced error by neglecting the contribution of the internal heat flux is, however, probably not too big. Since the effective temperature scales with the internal heat flux to the power of $(1 / 4)$, the effective temperature would not increase much. For e.g. Jupiter the difference between the equilibrium and effective temperatures is only 11 Kelvin (see Tables 4.2 and 6.3 in de Pater \& Lissauer, 2001).

Here I only intended to give a rough estimation of the effective temperatures of the companion candidates. A detailed description of irradiated planetary atmospheres, including internal heating, may be found in Guillot (2010). 


\subsection{Stellar evolutionary context}

\subsubsection{Common envelope evolution and final separation}

In order to estimate the behavior of a substellar companion in a common envelope of the expanding red giant as the progenitor of the subdwarf B star, we want to apply the scenarios explained in Soker (1998) and Nelemans \& Tauris (1998) to our substellar companion candidates. These models discriminate three different outcomes of planet-star interaction during the RGB phase: (i) evaporation or (ii) RLOF of the substellar companion, which both lead to a destruction and (iii) survival of the substellar object of the CE phase. Each scenario predicts a critical separation for evaporation $\left(a_{\text {eva }}\right)$, RLOF $\left(a_{R L O F}\right)$ and survival $\left(a_{\text {final }}\right)$. In the Soker (1998) models these are given as

- $a_{\text {eva }}=10 \cdot\left(m_{p} / M_{J}\right)^{-1} R_{\odot}$

- $a_{R L O F}=1.7 \eta\left(m_{p} / M_{J}\right)^{-1 / 3} R_{\odot}$

- $a_{f i n a l}=3 \alpha\left(m_{p} / 10 M_{J}\right)\left(R / 100 R_{\odot}\right)\left(M_{e n v} / 0.3 M_{\odot}\right)^{-1} R_{\odot}$

with $m_{p}$ being the companion mass, $\eta$ a dimensionless accretion parameter increasing the companion's radius (taken to be $<5$ ), $M_{\text {env }}$ the mass of the envelope, $\alpha$ an efficiency parameter of CE removal, taken to be between 0.2 and 0.3 (see later in the text) and $R$ being the giant star radius at the tip of the RGB.

The Nelemans \& Tauris (1998) models give the values as

- $a_{e v a}=\left[10 \eta\left(M_{J} / m_{p}\right)\right]^{1.18} R_{\odot}$

- $a_{R L O F}=(1 / 0.462) \eta r_{p}\left(M_{\text {core }} / m_{p}\right)^{1 / 3} R_{\odot}$

- $a_{\text {final }}=(\alpha \lambda / 2) \cdot M_{\text {core }} m_{p} /\left(M_{\text {tot }} M_{\text {env }}\right) \cdot R_{g}$

with $\eta, \alpha$ and $M_{e n v}$ as above and with $r_{p}$ being the planetary radius (assumed to be $0.1 R_{\odot}$ which is reasonable for ratios $m_{p} / M_{\odot}$ between 0.0001 and 0.08), $M_{\text {core }}$ being the core mass (assumed to be the canonical value of $\left.0.48 M_{\odot}\right), M_{\text {tot }}=M_{\text {core }}+M_{\text {env }}, R_{g}$ the red giant radius at the onset of the spiral-in phase and $\lambda$ a weighting factor $<1$ for the binding energy of the core and the envelope of the giant star, which is assumed to be 0.5 (see 
Nelemans \& Tauris, 1998).

Tidal interactions between the BD/exoplanet and the host star are believed to take place for separations roughly smaller than $5 \mathrm{AU}$ and the survival of a $\mathrm{CE}$ is predicted for critical companion masses bigger than roughly 1$10 M_{\text {Jup }}$ (Soker, 1998). A possible dependence of the CE removal efficiency parameter $\alpha$ on the secondary mass is investigated in Politano \& Weiler (2007). Zorotovic et al. (2010) in contrast suggest a universal value of the $\mathrm{CE}$ efficiency in the range of $\alpha=0.2-0.3$ with no indication for a dependence on the secondary mass.

Envelope masses $M_{e n v}$ of sdB stars are generally believed to be very small. Kawaler \& Hostler (2005) restrict the envelope mass to be smaller than $0.005 M_{\odot}$ for the bluest HB stars. In their extensive model calculations for the evolutionary tacks of subdwarf B stars, Charpinet et al. (2000, 2002a,,$\underline{b})$ use a core mass $M_{c}$ of $0.4758 M_{\odot}$ and envelope masses in the range [0.0002$0.0042] M_{\odot}$, while Kawaler \& Hostler $(2005)$ uses a core mass of $0.4797 M_{\odot}$ and envelope masses in the range [0.00001-0.00422] $M_{\odot}$.

The maximum radius of a sdB progenitor at the tip of the red giant branch is believed to be roughly 0.7 AU (Sweigart \& Gross, 1978), which corresponds to $150 R_{\odot}$. The measured final separation of the candidate HS $0702+6043$ b might imply that there was no $\mathrm{CE}$ evolution since its location is at $>0.7$ AU, but this is only true if a constant orbit is assumed. However, Soker (1998) predicts that for red giant radii bigger than $0.2 \mathrm{a}$, with a being the separation, tidal interaction will take place which would lead to a spiral-in (orbital decay) until a $\mathrm{CE}$ is formed. It will then depend on the domination of either inward drag due to friction or outward push due to envelope mass loss (lower binding energy) if the substellar companion further decreases the orbit and gets destroyed (by evaporation or RLOF) or if it will survive the CE phase, stop the inward drag and possibly even increase the orbit again due to the reduced binding energy. If we assume a maximum giant radius of the $\mathrm{sdB}$ progenitor of $150 R_{\odot}(0.7 \mathrm{AU}$, see above), all companions closer than $750 R_{\odot}(3.5 \mathrm{AU})$ will tidally interact and form a common envelope. In a more detailed study, Soker (1996) predicts that substellar companions of $0.001,0.01$ and $0.05 M_{\odot}$ will tidally interact with their RGB host stars for separations smaller than 2.0, 2.7 and 3.3AU, respectively, in an equilibrium 
tide model and for separations smaller than roughly $6 \mathrm{AU}$ for all substellar masses in a hydrodynamical model (see Soker, 1996, and references therein for details on these models). We therefore assume that both candidates, HS $0444+0458 \mathrm{~b}$ and HS $0702+6043 \mathrm{~b}$, have tidally interacted with the sdB progenitors on the RGB and probably also have undergone a $\mathrm{CE}$ phase which they have survived.

Let us recall our substellar companion candidate's parameters (here without errors):

- HS $0444+0458$ b (from f1): $m_{p}=29.28 M_{J}, a=58 R_{\odot}=0.27 A U$

- HS 0444+0458 b (from f2): $m_{p}=46.68 M_{J}, a=58 R_{\odot}=0.27 A U$

- HS 0702+6043 b (from f1): $m_{p}=5.77 M_{J}, a=253 R_{\odot}=1.177 \mathrm{AU}$

- HS 0702+6043 b (from f2): $m_{p}=5.32 M_{J}, a=241 R_{\odot}=1.120 A U$

For these, from top to bottom, we estimate $a_{\text {eva }}$ to be at $0.34,0.21,1.73$ and 1.88 Solar radii, respectively, in the Soker (1998) models and 1.88, 1.08, 12.78 and 14.07, respectively, in the Nelemans \& Tauris (1998) models. We find $a_{R L O F}$ values of $[2.76,2.36,4.74,4.87]$ Solar radii and $[2.77,2.37,4.76$, 4.89], respectively in the two models. Our measured final separations are all larger than these values, but this is of course expected since the candidates are post-RGB (post-CE) and therefore are known to have survived. Nevertheless it is nice to know that this is consistent with our estimations of $a_{e v a}$ and $a_{R L O F}$.

We now want to estimate which combinations of envelope mass and red giant radii yield the measured final separations $a_{\text {final }}$ according to the equations above. We do so for envelope masses spanning the whole expected range [0.0001-0.0080] $M_{\odot}$ and red giant radii up to $150\left(=R_{\max }\right)$ Solar radii. The results of these estimations are shown in Figures 7.2 and 7.3 . I want to emphasize that these are only 'order of magnitude' estimations and that there are several uncertainties and poorly constrained parameters $(\alpha, \eta, \lambda)$ included in the equations. However, with more empirical data like presented here it will hopefully be possible to build up significant statistics in order to put some tight constraints on these parameter.

The panels in Figure 7.2 show our estimations according to the Soker (1998) models for our candidates HS $0444+0458$ b (f1: top left, f2: top right) and 

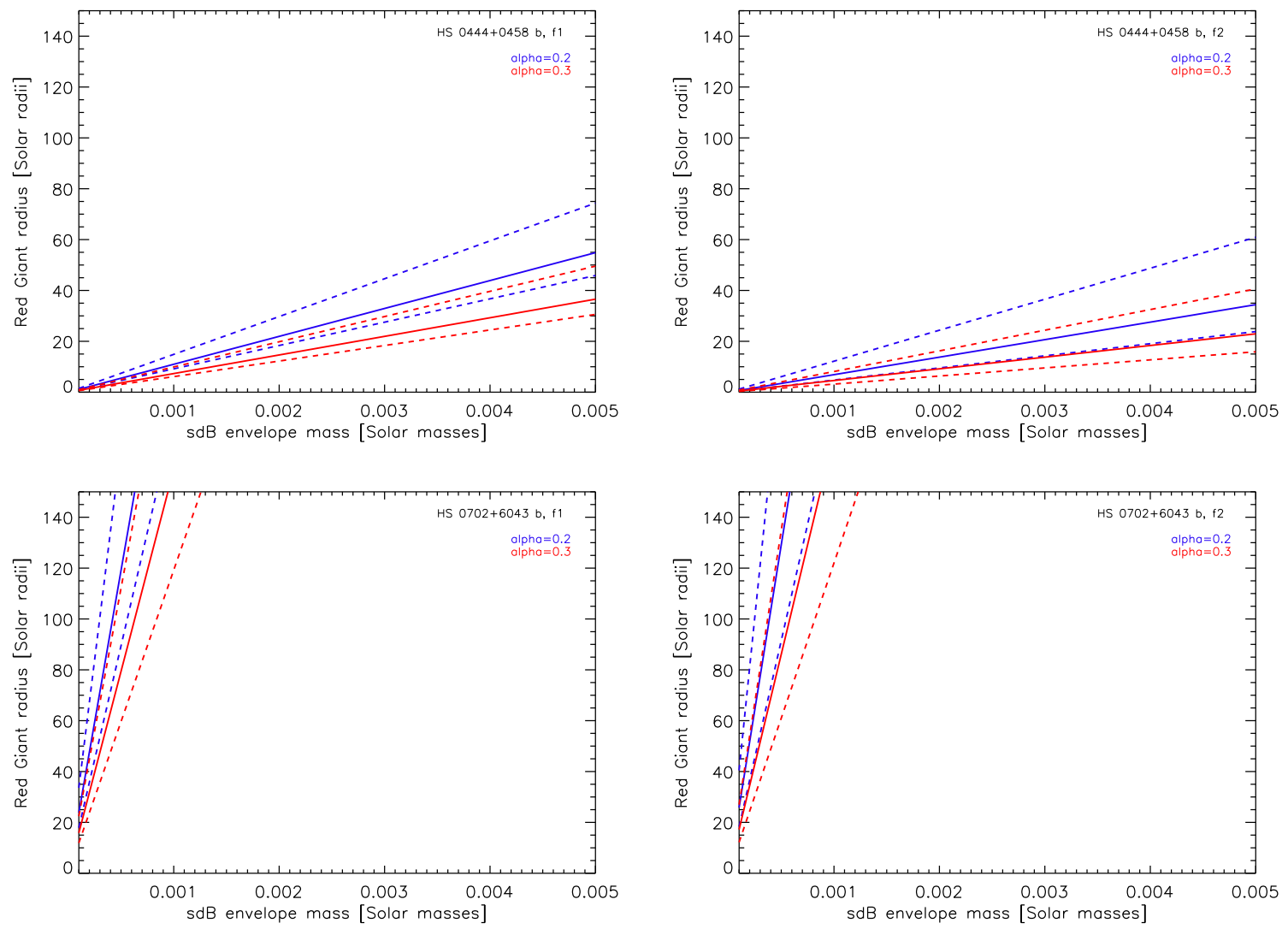

Figure 7.2: Combinations of envelope masses and red giant radii resulting in the measured final separation according to the Soker (1998) models. Top panels: based on f1 and f2 in HS 0444+0458. Bottom panels: based on f1 and f2 in HS 0702+6043. See text for details.

HS 0702+6043 b (f1:bottom left, f2: bottom right). The dashed lines account for the errors in the companion masses and the area between the dashed lines defines those combinations of envelope mass and red giant radii resulting in the correct (measured) final separation $a_{\text {final }}$. In each plot we use two different CE efficiency parameter: 0.2 (blue) and 0.3 (red), as already justified above and referring to Zorotovic et al. (2010). Combinations outside these triangular regions would yield final separations inconsistent with our results. Figure 7.3 shows the same as Figure 7.2, but for the Nelemans \& Tauris (1998) models.

What could we perhaps learn from these 'order of magnitude' estimations?

- HS 0444+0458: Figure 7.2 suggests that HS 0444+0458 should have envelope masses $\geq 0.0005 M_{\odot}$ because for lower masses the radius of the 

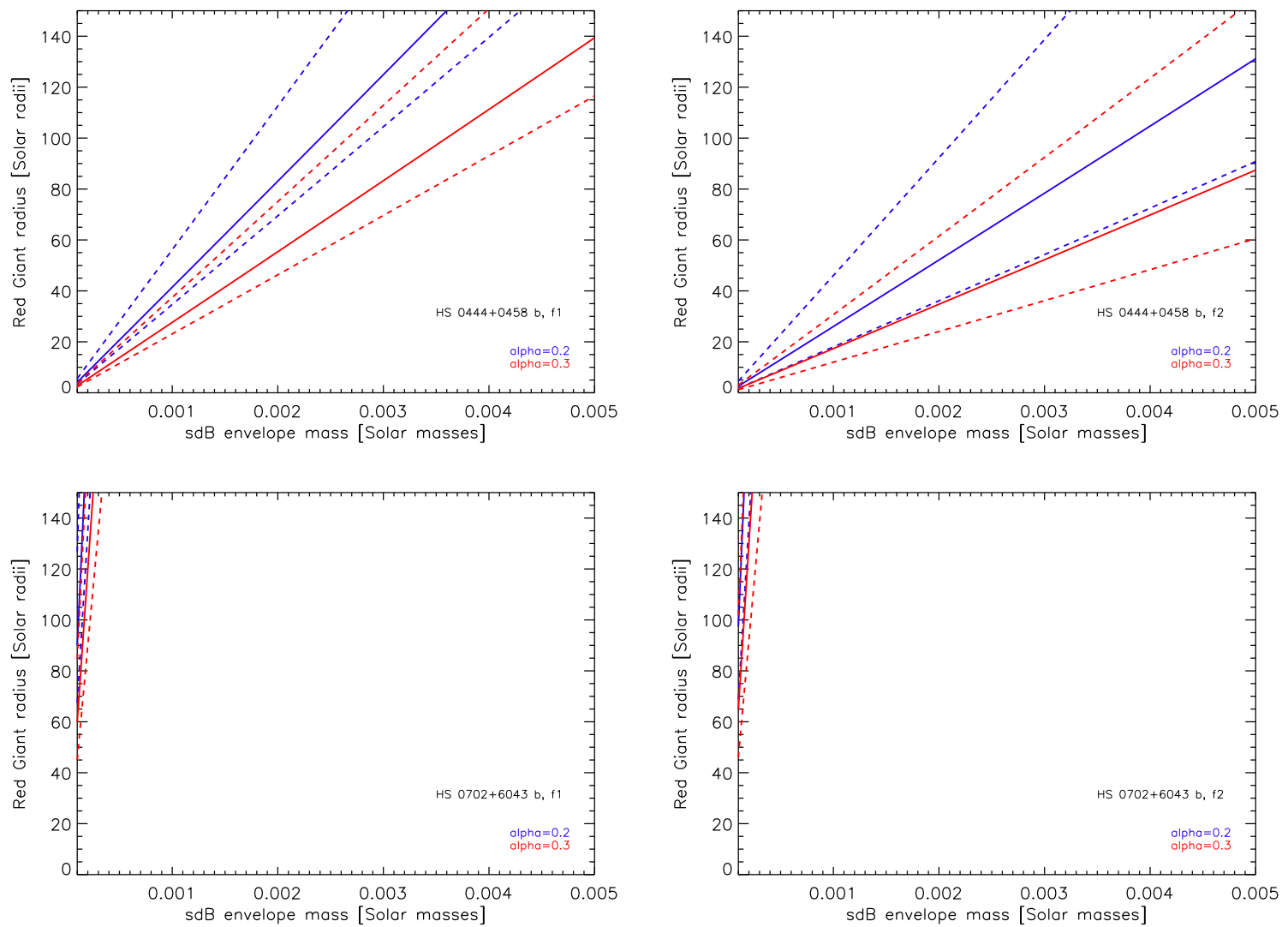

Figure 7.3: Same as Figure 7.2, but according to the Nelemans \& Tauris (1998) models. See text for details.

red giant becomes unrealistically small. In addition, the red giant radius might be estimated to be $<50 R_{\odot}$ since larger values would require envelope masses too large for sdB stars. However, this last point is not supported by Figure 7.3. The envelope mass can roughly be estimated to be $\geq 0.0003 M_{\odot}$ in the case of the models shown in Figure 7.3 .

- HS 0702+6043: As suggested by Figure 7.2, the envelope mass should be $\leq 0.001 M_{\odot}$ because higher envelope masses would yield red giant radii bigger than the maximum radius $^{3}$ of $150 R_{\odot}$. In addition, the red giant radius might be estimated to be $>20 R_{\odot}$ since smaller values would require envelope masses too small for sdB stars. The situation seems to be even more restricted in Figure 7.3 which suggests envelope masses $\leq 0.0003 M_{\odot}$ and red giant radii $>60 R_{\odot}$.

\footnotetext{
${ }^{3}$ As a reminder, it was stated earlier that the maximum radius of a sdB progenitor at the tip of the red giant branch is believed to be roughly $0.7 \mathrm{AU}$ (Sweigart \& Gross, 1978 ), which corresponds to $150 R_{\odot}$.
} 
Although these two models are not fully comparable to each other (red giant radius at the tip of the RGB versus red giant radius at the onset of the spiral-in phase), they still yield a similar general picture as outlined above, which allows to exclude certain regimes of envelope masses or giant radii. Or less strictly speaking: based on our measurements of the final separation, some regimes of envelope masses and giant radii are less probable than others.

These estimations of the possible ranges of envelope masses and red giant radii may not be very precise but can still be helpful in providing a consistency check or an input/constraints for asteroseismological models. If the envelope mass for our targets could be constrained via asteroseismological modeling, we could then give a statement on the giant expansion of the sdB progenitor when it was on the red giant branch. A nice example for such constraints on asteroseismic models is presented by Kawaler (2010) for the case of the sdB HS 2201+2610. Two different models, one with an envelope mass of $0.0020 M_{\odot}$ and the other with $0.0002 M_{\odot}$ fit the observed periods very well, but only the former one is able to fit also the measured $\dot{P}$ values. Hence, the envelope mass can be constrained quite well.

\subsubsection{Evolutionary status of the sdBs}

The evolutionary timescale $P / \dot{P}$ can be related to an expansion timescale $R / \dot{R}$ (see e. g. Kawaler, 2010). With $P \approx R^{3 / 2}(G M)^{-1 / 2}$ one gets $d \ln P / d t \approx$ $\frac{3}{2} d \ln R / d t$ and finally $\dot{P} / P \approx \frac{3}{2} \dot{R} / R$. This can be expressed as

$$
R_{\text {evol }} \approx \frac{3}{2} T_{\text {evol }}
$$

if we define $R_{\text {evol }}=R / \dot{R}$ and $T_{\text {evol }}=P / \dot{P}$ to be the expansion- and evolutionary timescales, respectively.

The relation $P \approx R^{3 / 2}(G M)^{-1 / 2}$ (which basically describes the dynamical timescale) can be obtained in the following way.

Assuming hydrostatic equilibrium $d p / d r \approx g(r) \cdot \rho(r)$ we may approximate the gravitational acceleration $g(r) \approx G M / R^{2}$ and the mass density $\rho(r) \approx$ $\langle\rho\rangle=M / V=3 M / 4 \pi R^{3}$ with the global properties total mass $M$ and total radius $R$. If we assume that the pressure only depends linearly on the radius, we may approximate $d p / d r \approx\langle p\rangle / R$ and via $\langle p\rangle / R \approx G M / R^{2} \cdot 3 M / 4 \pi R^{3}$ 


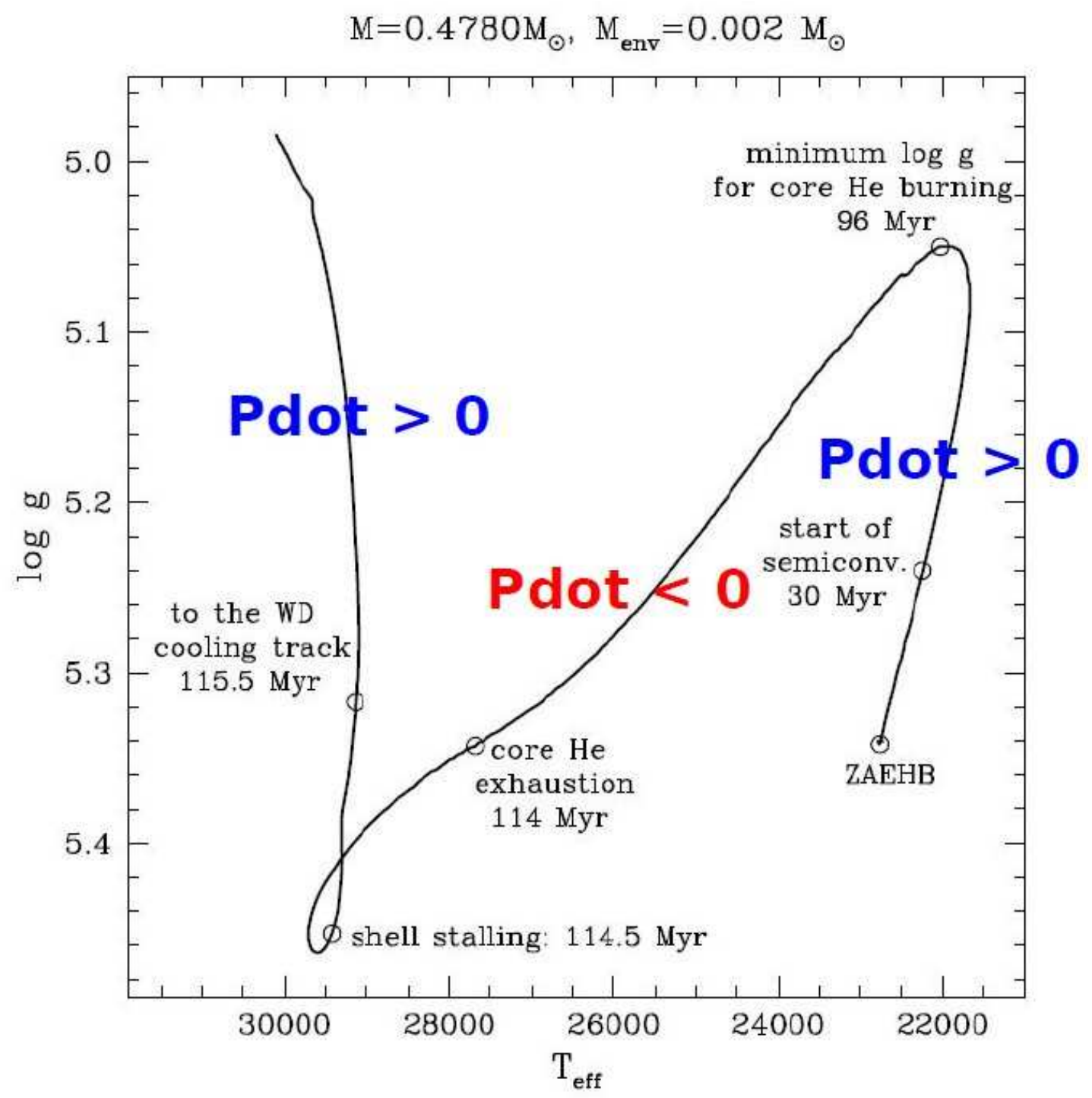

Figure 7.4: Typical evolutionary track for a model with the parameters given at the top. Time after ZAEHB and physical conditions at different stages are indicated. In addition, the secular period changes during different phases of evolution are labeled. Modified Figure from Kawaler (2010).

find an expression for $\langle p\rangle$, which is $\langle p\rangle \approx 3 G M^{2} / 4 \pi R^{4}$.

In order to be able to pulsate as a whole, a stars pulsational period $P$ must be at least as big as the one of a sonic wave crossing the whole star. This may be expressed as $P \approx R /\left\langle c_{S}\right\rangle$ with $c_{S}$ being the average sound speed in an ideal gas. With $c_{S}^{2}=\langle p\rangle /\langle\rho\rangle$ and inserting the above expressions we have $c_{S}=\sqrt{G M / R}$. This finally yields the relation $P \approx R^{3 / 2}(G M)^{-1 / 2}$. 


$$
\mathrm{M}=0.4780 \mathrm{M}_{\odot}, \mathrm{M}_{\text {env }}=0.002 \mathrm{M}_{\odot}
$$

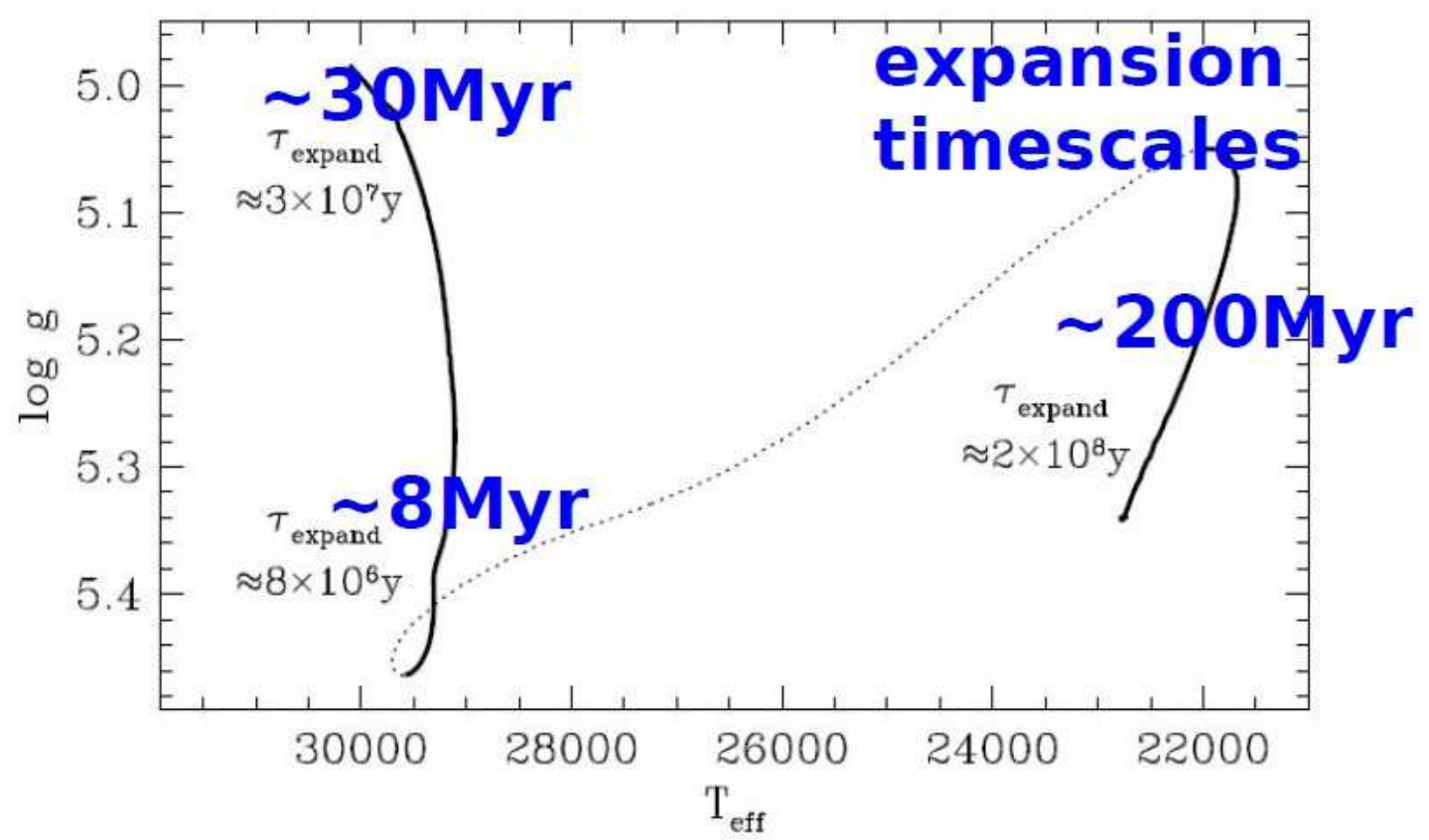

Figure 7.5: Typical expansion timescales for a model with the parameters given at the top. Modified Figure from Kawaler (2010).

Figures 7.4 and 7.5 show typical evolutionary tracks of a sdB star. According to Kawaler (2010) modifying the envelope thickness will result in a shift on the parameter plane but not change the overall appearance of the track. We can therefore assume the shape of the evolutionary track as a 'general' one for sdB stars. After the zero age extreme horizontal branch (core helium ignition), the sdB star will first experience a phase of expansion, accompanied by a steady increase in the pulsation periods, i.e. a positive $\dot{P}$, as indicated in Figure 7.4. The increase in the radius is connected to a decrease in surface gravity, such that the path of the evolutionary track leads 'upwards' in the log g - T eff diagram. Roughly 100 Myr after the ZAEHB the energy produced in the core begins to decrease, owing to the reduction of the thermonuclear fuel density (i.e. the reduction of helium supply). At that point of minimum log g for core helium burning, which is also the turning point from positive to negative $\dot{P}$, the core is fully convective and the energy generation is still maximum, although the helium abundance has dropped to roughly 0.12 (Kawaler, 2010). Since the energy production from 
now on is less efficient the star reacts with a phase of contraction, accompanied by an increase of surface gravity and resulting in a 'downward' path in the $\log g-\mathrm{T}_{\text {eff }}$ which is also characterized by a negative $\dot{P}$. This phase is much faster than the previous one and lasts until roughly 115 Myr after the ZAEHB. It includes the core helium exhaustion and is ended by the onset of helium shell burning. This point also marks the end of the EHB evolution. At this second turning point (from a negative $\dot{P}$ back to a positive) after core helium exhaustion the energy generation rate peaks in a helium shell surrounding the $\mathrm{C} / \mathrm{O}$ core. The subsequent helium fusion in the shell is accompanied by an increase of the radius and therefore a phase where the periods are increasing again (i.e. a positive $\dot{P}$ ). The star is now on its post-EHB way heading towards the sdO regime and finally the white dwarf cooling sequence. An interesting aspect appears when looking at the region in Figure 7.4 where the track crosses the same parameter combination twice (the loop at $\log g$ of 5.4 and effective temperature of roughly $29000 \mathrm{~K}$ ). The spectroscopic degeneracy of that point could be lifted by a measurement of $\dot{P}$ which could, by its sign, unambiguously tell if a star at that point will increase or decrease its surface gravity. This discrimination can not be made with spectroscopy, but only with a $\dot{P}$ measurement.

A very extensive description of the various stages in sdB evolution and in particular the consequences of evolutionary aspects on the pulsation periods is provided in Charpinet et al. (2002b). The basic scenario sketched above is in principle valid for both, acoustic and gravity modes. The evolution of the latter, however, is a bit more complicated since these are sensitive to both the surface gravity and the effective temperature. The acoustic modes, in contrast are primarily only sensitive to the surface gravity. Details may be found in Charpinet et al. (2002b).

Figure 7.5 illustrates the typical expansion timescales during the various evolutionary stages outlined above. The expansion timescales derived for our targets are $0.7 \mathrm{Myr}$ and $2.7 \mathrm{Myr}$, respectively, for the frequency $\mathrm{f} 1$ and f2 of the target HS $0444+0458$ and $60.2 \mathrm{Myr}$ and 3.3 Myr, respectively, for f1 and f2 in HS 0702+6043. These values are also listed in Table 6.1 along with the evolutionary timescales.

Let me also point out that all measured $\dot{P}$ values of the frequencies of our targets are positive. Considering Figure 7.4 we can already state that our 
targets are either in their first stage of evolution prior to the reduction of thermonuclear fuel or they are already evolving off the EHB after core helium exhaustion. If we additionally look at the typical expansion timescales given in Figure 7.5 and compare these to our measurements, we can state that the values are more consistent with the shorter ones (8 Myr, $30 \mathrm{Myr}$ ) at the end of EHB evolution instead of the 200 Myr timescale in the first stage. Combining these two informations, we may conclude that our targets are probably already evolving off the extreme horizontal branch and have already experienced a core helium exhaustion. Such information can be valuable as an input for or discrimination between different seismological models. In the case of HS $2201+2610$ the measurement of $\dot{P}$ helped to favor one particular model over the other and to pin down the evolutionary status (Kawaler, 2010). This is of course also desirable for our targets HS 0702+6043 and HS 0444+0458 and left for further studies. A similar idea is provided in Figure 8 of Charpinet et al. (2002b), where the authors relate the behavior of the mean relative rate of period change to the effective temperature and the surface gravity. The age of a sdB star is not a priori known. But a precise measurement of the rate of period change could then be used (in combination with accurate estimates of the effective temperature or the surface gravity) to give proper estimates on the age.

Let us summarize these considerations on evolutionary aspects. Following Heber (2009), Charpinet et al. (2002b) and Kawaler (2010) the theoretical timescales for core helium burning (i.e. EHB lifetime) and post-EHB evolution are $\sim 10^{8} \mathrm{yr}(\sim 100 \mathrm{Myr})$ and $\sim 3 \cdot 10^{7} \mathrm{yr}(\sim 30 \mathrm{Myr})$, respectively. The measured evolutionary timescales for the periods under consideration in our targets HS $0444+0458$ and HS 0702+6043 suggest that these objects are already evolving off the extreme horizontal branch. In the literature there only exist two more sdB targets with published $\dot{P}$ measurements. One is HS $2201+2610$, which has already been discussed in this thesis. To recapitulate, the $\dot{P}$ values and evolutionary timescales for the two strongest frequencies are $1.46 \cdot 10^{-12}(7.6 \mathrm{Myr})$ and $2.05 \cdot 10^{-12}(5.5 \mathrm{Myr})$, respectively. The other one is the sdB star CS 1246. Barlow et al. (2011b) measure a $\dot{P}$ of $-1.9 \cdot 10^{-11}$ for the dominant $371.7 \mathrm{~s}$ pulsation period, translating to an evolutionary timescale of roughly $0.62 \mathrm{Myr}$. Note the negative value of $\dot{P}$ which suggests that CS 1246 is approaching the end of its life as an EHB star (also 
see Figure 7.4). Interestingly, Barlow et al. (2011b) who applied a timing method based on a huge photometric data set of CS 1246 and conducted an $\mathrm{O}-\mathrm{C}$ analysis, also detect a sinusoidal variation in the $\mathrm{O}-\mathrm{C}$ diagram of the pulsation mentioned above. This signature could be produced by the presence of a low-mass stellar companion with a minimum mass of $0.12 M_{\odot}$ orbiting the sdB at a distance of only $20 R_{\odot}$. This is therefore also a very promising target for considerations of sdB formation scenarios. Even more, Barlow et al. (2011a) gathered follow-up spectroscopy for CS 1246 and indeed found RV variations corresponding to these being expected based on the photometric $\mathrm{O}-\mathrm{C}$ timing analysis. The $\mathrm{O}-\mathrm{C}$ analysis predicted $\mathrm{RV}$ variations of $16.6 \mathrm{~km} / \mathrm{s}$ and these were, within the errors, confirmed by Doppler measurements.

There exist many measurements of secular changes of the orbital period in binary systems. However, measurements of secular changes of the pulsation period of a pulsating single star are rare. Beside the $\dot{P}$ measurements for sdB stars (HS 2201+2610, CS 1246, HS 0444+0458, HS 0702+6043), secular period changes due to evolutionary effects have also been measured for some other pulsating stars. Examples are dwarf Cepheids (Percv et al., 1980) or the pulsating white dwarfs G 117-B15A (Kepler et al.. 2005). GD66 (Mullally et al., 2008, 2009) and EC 20058-5234 (Dalessio et al., 2011). Pulsating white dwarfs show rates of secular period changes of the order of $10^{-12}$ to $10^{-15}$. After the pulsars (roughly $10^{-12}$ to $10^{-21}$ ) the white dwarfs and sdBs provide the most stable astrophysical clocks known today.

To provide a descriptive picture of what such values of $\dot{P}$ mean, I want to put them in another way. The measured secular period changes of our targets can be expressed as follows: the main pulsational period f1 in HS 0444+0458 increases with a rate of one second per 3400 years and the second pulsation f2 with a rate of one second per 10500 years. In HS 0702+6043 the main and second pulsational periods, $\mathrm{f} 1$ and $\mathrm{f} 2$, increase with rates of one second per 110000 years and one second per 5700 years, respectively. At first sight it seems odd that there is a quite big difference in the rates of period change between the main and second pulsation periods (especially evident in HS 0702+6043). But one has to keep in mind that these rates, besides the 
age since the ZAEHB, mainly depend on the mode degree and radial order (see e.g. Table 3A in Charpinet et al., 2002b). Even modes with comparable periods may have quite different rates of period change, depending on their degree and radial order. To attempt to determine these mode parameter for our targets should be subject of a future investigation.

The case of the pulsating white dwarf EC 20058-5234 (Dalessio et al., 2011) shows a similar situation: The $\dot{P}$ values of the four pulsations considered differ by more than an order of magnitude. Even more extreme than in our case, two pulsations in EC 20058-5234 show a positive $\dot{P}$ while the other two show a negative one. To try to better understand these features, more actual measurements of $\dot{P}$, both for pulsating sdB stars and pulsating white dwarfs, are required.

\subsubsection{Impact of the companion candidates on sdB evolution}

The most interesting aspects of the detection of the HS 0444+0458 b brown dwarf candidate are its parameters mass and separation: the close separation of only $0.27 \mathrm{AU}$ strongly suggests a common envelope phase and the mass of the companion candidate places it into the so-called "brown dwarf desert". The brown dwarf desert is commonly referred to as a region in the 'companion mass versus orbital period plane' where there is a lack of brown dwarfs at separations below 5 AU (see Figure 5 in Grether \& Lineweaver, 2006, for a nice illustration of the brown dwarf desert). A possible explanation might be enhanced inward migration and swallowing of brown dwarfs in the early protoplanetary disk (e.g. Armitage \& Bonnell, 2003). However, one has to keep in mind that the concept of a brown dwarf desert is still under debate and the characteristics as outlined above are drawn for solarlike host stars. It is not known how this concept translates to hot subdwarf host stars. Still, it is interesting to know that HS $0444+0458$ b would not have been expected to be able to exist at its current location if the host star were solar-like.

Concerning the common envelope aspect, HS $0444+0458 \mathrm{~b}$ might be comparable to the brown dwarf companion of the white dwarf WD 0137-349 (Maxted et al., 2006). In this system the brown dwarf has a much closer separation and is proven to have survived a common envelope phase with the white dwarf progenitor. The survival of a common envelope phase ba- 
sically means that the companion was sufficiently massive enough to force the envelope to partly co-rotate with its orbit and therefore stopping the inward drag and spiraling in towards the core of the red giant.

The presence of HS $0444+0458 \mathrm{~b}$ suggests that it was able to enhance the red giant's mass loss by deposition of angular momentum to the envelope but still surviving this phase and stopping an inward drag. It is therefore a strong support for the hypothesis that even substellar companions are able to trigger an enhanced mass-loss during the red giant phase, such that it can result in the formation of a single subdwarf B star.

Unlike the HS $0444+0458$ system, a direct common envelope evolution of the planetary candidate HS $0702+6043 \mathrm{~b}$ is, due to its larger separation of roughly $1.15 \mathrm{AU}$, harder to imagine. Nevertheless we have outlined earlier that a tidal interaction with the red giant envelope is very probable. This system, in terms of companion mass and separation, more resembles the HS 2201+2610 system where the planet of 3.2 Jupiter masses has a separation of 1.7 AU. During the discussion of possible formation scenarios of the latter system, several aspects have been addressed in Silvotti et al. (2007) and Silvotti (2008), which might also apply to HS 0702+6043 b:

As described earlier, two competing processes determine the orbital evolution. Stellar mass loss tends to increase the orbit due the lower binding energy whereas tidal interaction tends to reduce the angular momentum and therefore to decrease the orbit, hence a spiraling-in of the companion (still one has to keep in mind that neither stellar mass loss nor tidal interaction are well-understood processes). However, one possible scenario for the HS $0702+6043$ system is that the tidal interaction was insufficient to build up a common envelope phase. In that case, the orbit of HS $0702+6043 \mathrm{~b}$ would have been tighter in the past and, owing to the strong stellar mass loss, pushed outwards to its current location at roughly 1.15 AU. Yet another scenario might be the following: If the star at some point would have filled its Roche lobe as the companion was sufficiently close, mass transfer would have started. The planet spirals to the outer layers of the giants atmosphere where accretion disrupts and spiraling-in due to accretion stops. In this scenario, spiraling-in due to friction must be sufficiently low such that the inward migration stops and the planet can survive. Also, HS 0702+6043 
b could possibly be a tracer for a further object which was closer-in and which triggered an enhanced mass loss but was destroyed in the process. This is of course highly speculative and cannot be proven by any kind of data if one assumes that the close-in object was totally destroyed during a CE phase and did not form some kind of a long-lived disk.

Finally, a merger scenario as described in section 1.2.1 cannot be ruled out completely. In such a case the companions would be of "second generation". If double-lined emission peaks as signatures for a gaseous disk which would have formed during the merging process could be excluded by high-res spectroscopy, it would be a strong argument against this alternative merger scenario. This only holds if one assumes that a hypothetical disk would exist long enough after the merging scenario to be measured.

\subsubsection{Future of solar-like host stars}

Most currently known exoplanet host stars are non-evolved and situated on the main sequence. It is interesting to ask the question what will happen to these, and in particular to their exoplanets, once they evolve off the main sequence and towards the red giant branch. Looking at the distribution of orbital separations, one will find that the vast majority of the currently known exoplanets orbit their host star at rather close orbits: roughly $75 \%$ closer than $2 \mathrm{AU}, 55 \%$ closer than $1 \mathrm{AU}$ and even $33 \%$ closer than $0.1 \mathrm{AU}$ (still one has to keep in mind that these numbers are biased towards selection effects owing to the sensitivities of the detection methods). If we apply the criterion that tidal interaction between the exoplanet and the expanding red giant will take place once the giant radius exceeds a fifth of the orbital separation (see section 7.3.1) and consider the red giant radial expansion of a typical solar-like star, one can predict that a great majority of the current exoplanet sample will tidally interact with their host star. Since many of these close-in exoplanets are also quite massive (about half of the planets which are closer than $2 \mathrm{AU}$ are more massive than one Jupiter mass), it is therefore valid to assume that these will be able to enhance the mass loss of their host stars significantly. If this "planet-induced" mass loss is strong enough, a major part of the stellar envelope may be removed prior to the helium flash. Hence many of the exoplanet host stars known today could then, 
in several billion years, become objects on the extreme horizontal branch and not reach the AGB at all!

The hypothesis that many of the non-evolved exoplanet host stars could become single sdB stars is hard to corroborate but, if correct, most of the single sdB stars should have substellar companions. The high detection rate of substellar companions to sdB stars, to which this thesis significantly contributes, is an observational fact in accordance with this hypothesis. Of course it is desirable to increase the current statistics and find more substellar companions to sdB stars. One also has to keep in mind that only such non-evolved exoplanetary systems which have planets massive and close enough to trigger an enhanced mass loss will have the chance to become systems with a subdwarf B host star.

The exoplanet population around sdB stars is expected to be quite different from the "non-evolved" population. Low mass planets (roughly $<1 M_{J u p}$ ) should not be able to exist very close to sdB stars since they would probably be destroyed during the common envelope phase. Low mass planets in wide orbits $(>5 \mathrm{AU})$ around sdB stars were probably insufficient to trigger an enhanced stellar mass loss but may still be an important tracer for further planets closer in (multiple systems are not that rare). Higher mass planets should primarily be searched in close orbits around single sdB stars since these are the ones with the highest ability to form the sdBs.

The hypothesis outlined above should be considered in studies concerning the long-term evolution of planetary systems in general and the evolution of current exoplanet host stars in particular.

The future evolution of exoplanet host stars of course also leads to the question of the final destiny of the Sun and the Earth. There exist several studies of the long-term evolution of the Solar System (e.g. Rvbicki \& Denis, 2001; Rasio et al., 1996; Duncan \& Lissauer, 1998; Villaver, 2011). It is expected that life ${ }^{4}$ on Earth will extinguish already during the solar main sequence evolution when the habitable zone, owing to the increasing solar luminosity, moves considerably outwards beyond Earth's orbit. The fate of the Earth as a celestial body is, however, still a matter of debate. I will briefly summarize some of the statements given in the studies cited above.

\footnotetext{
${ }^{4} \mathrm{At}$ least in a form as we currently know or define it.
} 
The Sun will increase its luminosity and radius by factors of roughly 2100 and 150 times the present value, respectively, once it reaches the tip of the red giant branch. The effective temperature will drop to about $3200 \mathrm{~K}$ or less. It is suggested that tidal interaction has to be taken into account only for Mercury, Venus and Earth. Tidal interaction is proportional to the planetary mass but also proportional to the inverse distance by a power of eight. Hence, tidal interaction is negligible for Mars and the Solar System Giants (see details in Rybicki \& Denis, 2001). It is expected that Mercury will be evaporated when the Sun is on the red giant branch and that Venus is most probably also destroyed while Mars will most probably survive. The giant planets Jupiter, Saturn, Uranus and Neptune will survive all giant stages of the Sun, both RGB and AGB, and expand their orbits due to the solar mass loss by about $80 \%$ if the final mass of the sun is assumed to be $0.55 M_{\odot}$. A crucial aspect in the ultimate fate of the Earth seems to be the value of the solar radius on the red giant branch. If it reaches a large value close to $1 \mathrm{AU}$ and if the mass loss will be moderate, then the Earth will probably be swallowed and destroyed. If, in contrast, the solar radius remains smaller throughout the red giant branch then the tidal interaction may be negligible and the Earth would increase its orbit and survive the RGB phase. Even more speculative is Earth's fate during the phase of thermal pulses when the Sun would be on the asymptotic giant branch.

Compared to the studies mentioned above, Schröder \& Connon Smith (2008) use a different mass-loss relation. In their study Earth will be engulfed by the sun prior to the RGB-tip and it is estimated that the minimum orbital distance to avoid engulfment by the expanding sun would be $1.15 \mathrm{AU}$. Although these considerations are very speculative and the involved physics are poorly understood (e.g. stellar mass loss, tidal interaction, common envelope evolution), the questions raised are nevertheless highly interesting. A final answer will probably have to wait another several billion years. 


\section{Chapter 8}

\section{Summary and outlook}

The most important steps during the reduction and analysis of the data and the interpretation of the results will be summarized in the following section. An outlook will conclude this thesis.

\subsection{Summary}

The search for substellar companions to single sdB stars was declared to be a major goal of this thesis along with the direct measurement of evolutionary timescales. This was justified with the closely related question if substellar companions could be able to influence the evolution of their host star or even be responsible for their formation in the first place. The method applied to achieve these goals was a timing method (here: $\mathrm{O}-\mathrm{C}$ analysis) based on the arrival times of intensity maxima due to the pulsations of the target stars. The required type of data to conduct the analysis was time-series photometry and the data were gathered as multi-site observations, comprised of a number of small- to medium-size ground based observatories. The data summed up to 949 hours of photometry on the target HS 0702+6043 and 99 hours on the target HS $0444+0458$. The importance of time accuracy was addressed and all data were transformed to the same data format to ensure consistency between different data sets. This included to transform all data to a barycentric reference frame in the same time standard. The subsequent analysis was performed with weighted data to account for the different qualities of data sets taken with different instruments. The frequency analysis of both targets suggested that a full $\mathrm{O}-\mathrm{C}$ procedure could be conducted independently for two distinct pulsational frequencies, f1 and 
$\mathrm{f} 2$, in each target. We identified one component in each of the $\mathrm{O}-\mathrm{C}$ diagrams with an evolutionary origin. Based on this component it was possible to directly measure the secular evolution of the pulsation periods $(\dot{P})$ and hence to derive the evolutionary timescales. These resulted to be $0.466 \mathrm{Myr}$ and 1.785 Myr, respectively, for the two frequencies analyzed in HS 0444+0458 and 40.110 Myr and 2.181 Myr, respectively, for the two frequencies analyzed in HS 0702+6043. These values, compared to evolutionary models, were then used to constrain the evolutionary status of the two sdB stars. Together with the conclusion that both targets are in an expansion phase (based on the sign of $\dot{P}$ ), these results suggest that both targets have already experienced core helium exhaustion and are therefore close to the end of their lifetime as an object on the extreme horizontal branch and developing towards the white dwarf cooling sequence.

Another component in the $\mathrm{O}-\mathrm{C}$ diagrams was interpreted with a beating effect of unresolved closely spaced frequencies. We concluded these $\mathrm{O}-\mathrm{C}$ signatures to belong to beat periods of roughly $840 \mathrm{~d}$ and $195 \mathrm{~d}$, respectively, for the two frequencies analyzed in HS0444+0458 and $911 \mathrm{~d}$ and $76 \mathrm{~d}$, respectively, for the two frequencies analyzed in HS 0702+6043. Furthermore, both targets showed signatures in their $\mathrm{O}-\mathrm{C}$ diagrams which we interpreted with an orbital origin, i.e. the presence of a companion. The characteristics of these signatures allowed us to derive the minimum masses and orbital separations of the companion candidates. These suggest that the companion candidate to HS 0444+0458 is most likely a Brown Dwarf and the candidate to HS 0702+6043 most likely an exoplanet. We termed these as HS $0444+0458 \mathrm{~b}$ and HS $0702+6043 \mathrm{~b}$, respectively.

The claim that these signatures are indeed due to companions is supported by the fact that we find similar results, within the error limits, independently and consistently in the two frequencies considered in each target. The Brown dwarf candidate HS $0444+0458$ b has a minimum mass of $\left(29.284_{-7.674}^{+7.735}\right) M_{\text {Jup }}$ based on $\mathrm{f} 1$ and $\left(46.675_{-20.299}^{+20.733}\right) M_{\text {Jup }}$ based on $\mathrm{f} 2$ and orbits the host star HS $0444+0458$ at a distance of $(0.269 \pm 0.001) \mathrm{AU}$ or $\left(0.270_{-0.003}^{+0.002}\right) \mathrm{AU}$, respectively. The orbital periods are $(72.00 \pm 0.43) \mathrm{d}$ and $(72.32 \pm 1.14) \mathrm{d}$, respectively. These results are consistent within the error bars. The exoplanet candidate HS $0702+6043 \mathrm{~b}$ has a minimum mass of $\left(5.770_{-1.684}^{+1.912}\right) M_{J u p}$ based on $\mathrm{f} 1$ and $\left(5.317_{-1.922}^{+2.189}\right) M_{J u p}$ based on $\mathrm{f} 2$ at a distance of $\left(1.177_{-0.072}^{+0.070}\right) \mathrm{AU}$ or 
$\left(1.120_{-0.070}^{+0.069}\right)$ AU, respectively, from its host star HS 0702+6043. The orbital periods are $(659.73 \pm 59.58) \mathrm{d}$ and $(612.59 \pm 57.19) \mathrm{d}$, respectively. These results, based on the two independent frequencies, are again consistent within the error bars. Since the results derived for f1 and f2 are independent in each target, we can calculate weighted means. These finally yield

$\mathrm{a}=(\mathbf{0 . 2 6 9} \pm \mathbf{0 . 0 0 1}) \mathrm{AU}$ and $\mathrm{m} \cdot \operatorname{sini}=(31.41 \pm 7.25) \mathrm{M}_{\mathrm{Jup}}$

for HS $0444+0458$ b. For HS $0702+6043 \mathrm{~b}$ one gets

$\mathrm{a}=(\mathbf{1 . 1 4 8} \pm \mathbf{0 . 0 5 0}) \mathrm{AU}$ and $\mathrm{m} \cdot \operatorname{sini}=(\mathbf{5 . 5 8} \pm \mathbf{1 . 4 4}) \mathrm{M}_{\mathrm{Jup}}$. Due to the unknown inclination, which cannot be revealed with the timing method, the masses given above are minimum masses. Assuming a random distribution of orbital inclinations, it was estimated that HS $0444+0458 \mathrm{~b}$ and HS $0702+6043 \mathrm{~b}$ are substellar with high probabilities of $81.2 \%$ and $99.7 \%$, respectively. Another signature in the $\mathrm{O}-\mathrm{C}$ diagrams of HS $0702+6043$ shows some similar sinusoidal characteristics but cannot be interpreted as of orbital origin, since the results are not consistent between the two frequencies considered. This particular feature has to be investigated further. All the different components which we find to be present in the $\mathrm{O}-\mathrm{C}$ diagrams of HS 0444+0458 and HS 0702+6043 are summarized in Figure 8.1. In a subsequent step, the effective temperatures of the companion candidates were estimated to be approximately $\left(1216_{-158}^{+184}\right) \mathrm{K}$ for HS $0444+0458 \mathrm{~b}$ and $\left(572_{-57}^{+61}\right) \mathrm{K}$ for HS $0702+6043 \mathrm{~b}$. This estimation included the assumption that the internal heat flux is negligible compared to the incoming stellar flux and the assumption of a Jupiter-like bond albedo for both companion candidates. As a further implication, the measured final separation of the companion candidates was used in two models to estimate those combinations of red giant radii and envelope masses which yield consistent results with our measurements. If asteroseismic modeling for the two targets could give a proper estimation of the envelope mass, it would then be possible to put tight constraints on the red giant expansion radius. Finally, different formation scenarios for the systems HS 0444+0458 and HS 0702+6043 were presented and discussed. The conclusion was drawn that both substellar companion candidates have had the potential to trigger the enhanced mass loss of their host stars and therefore are a direct support for the sdB formation scenario which includes substellar companions as one possible formation channel. 

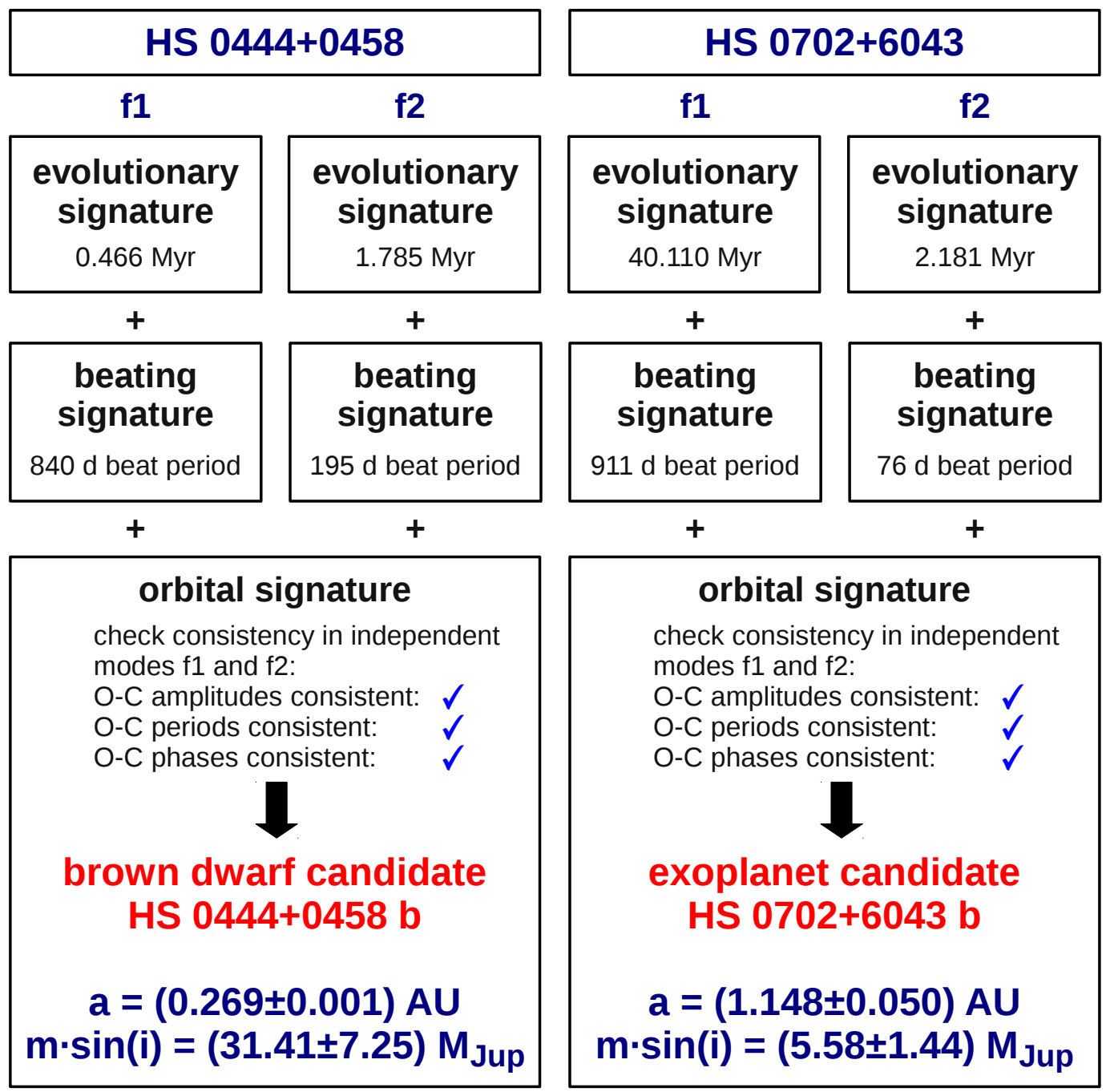

f1 f2
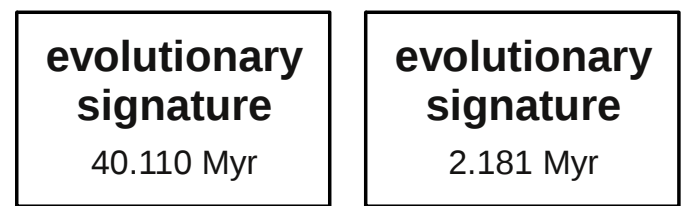

$+$

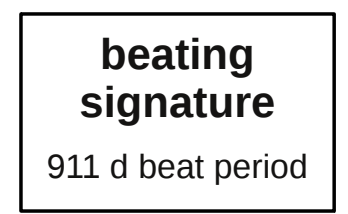

$+$

\begin{tabular}{|c|}
\hline beating \\
signature \\
$76 \mathrm{~d}$ beat period \\
\hline
\end{tabular}

$+$

$+$

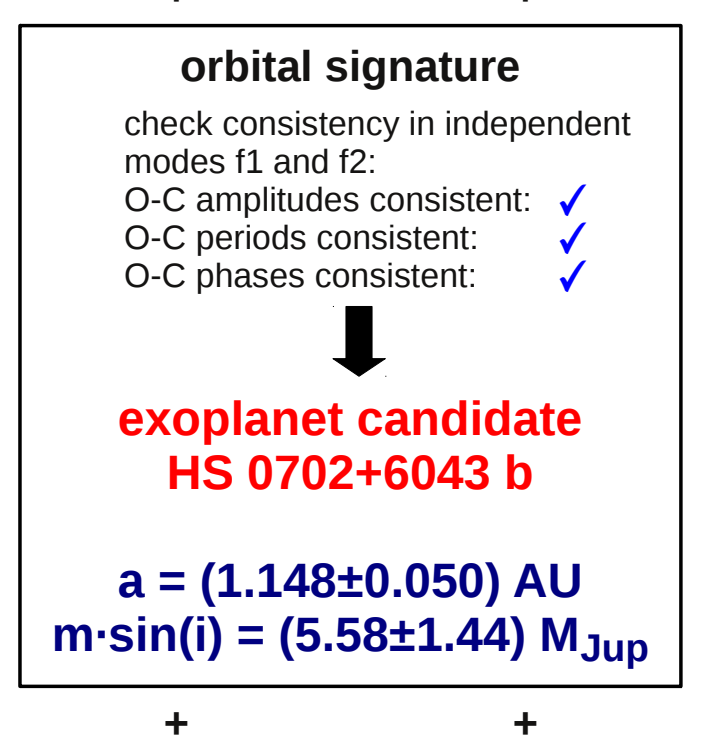

unknown sinusoidal signature

check consistency in independent modes $\mathrm{f} 1$ and $\mathrm{f} 2$ :

O-C amplitudes consistent:

$\mathrm{O}-\mathrm{C}$ periods consistent:

$\mathrm{O}-\mathrm{C}$ phases consistent:

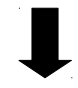

- orbital signal unlikely - signature has to be investigated further

Figure 8.1: Summary of the components found in the $\mathrm{O}-\mathrm{C}$ diagrams of the frequencies f1 and f2 in the targets HS 0444+0458 and HS 0702+6043. 


\subsection{Outlook}

Beyond the scope of the present thesis, there are several ideas on how our results could be tested and confirmed. The most immediate approach would be a search for the radial velocity signals which would be induced by the companion candidates. These RV signals have been predicted and quantified in section 7.2.3. A detection of these predicted signals would be a direct proof of our companion candidates, although it seems observationally challenging as we have discussed earlier.

Concerning the $\mathrm{O}-\mathrm{C}$ diagrams, an extension of the total time baseline will always result in an improvement. The frequency resolution will become better, error bars become smaller and the current orbital solution could be tested/improved by the inclusion of further $\mathrm{O}-\mathrm{C}$ points. We suggest to define PG 1325+101 and EC 09582-1137 as the highest priority targets for further EXOTIME observations in order to produce first reliable $\mathrm{O}-\mathrm{C}$ diagrams. Still it is desirable to keep the remaining EXOTIME objects (where thorough $\mathrm{O}-\mathrm{C}$ analyses are available and substellar companions have been found: HS 2201+2610, HS 0444+0458 and HS 0702+6043) as lower priority targets with the goal to get at least some $\mathrm{O}-\mathrm{C}$ points per year in order to refine the current $\mathrm{O}-\mathrm{C}$ solutions. In the case of HS $0702+6043$ it is also possible to "go back" in the $\mathrm{O}-\mathrm{C}$ diagram. The analysis presented in this thesis includes data from Nov 2007 until Nov 2010. As can be seen e.g. in Figure 4.4 there are also data available from the years 2005 and earlier. We had already estimated the maximum allowed gap between observations to avoid a cycle count error to be roughly 1100 days in the case of our current $\mathrm{O}-\mathrm{C}$ data on HS $0702+6043$. Hence we have by now accumulated enough data such that the HS 0702+6043 data from January 2005 could be safely added to our present $\mathrm{O}-\mathrm{C}$ analysis in a further study.

Additional observations of the EXOTIME targets in the IR wavelength would be desirable to strengthen our conclusion that these are indeed single sdB stars without any stellar companion. Such observations could also be considered in the context of gaseous disks. A non-detection would not completely rule out a merging process but it could make it unlikely (scenario mentioned in section 7.3 .3 and 1.2.1).

An effort to try to derive the unknown inclination with the final goal to calculate a true mass instead of a minimum mass has already been introduced 
in section 6.5 .1 with the example of HS $2201+2610$.

Further studies not directly connected to the presence of the companions but with the goal to further characterize the host star or its pulsations may also be conducted. Approaches to determine the mode parameter of the pulsations in HS $0444+0458$ and HS $0702+6043$ are multicolor photometry, time-resolved spectroscopy or a period matching with asteroseismic models. A search for systematic splittings in the periodogram could be conducted to infer the rotational periods of the stars.

This outline of desirable follow-up studies and follow-up observations shows that there still can be done a lot. A continuation of gathering $\mathrm{O}-\mathrm{C}$ points in order to confirm the current $\mathrm{O}-\mathrm{C}$ solutions is of course desirable. However, I want to point out that in the context of the main results of this thesis, namely the detection of the companion candidates HS 0444+0458 b and HS $0702+6043 \mathrm{~b}$, the most promising and most important task to do is the search for the predicted RV signals. 


\section{Bibliography}

Aerts, C., Christensen-Dalsgandd, J. \& Kurtz, D. W. (2010) Asteroseismology.

Armitage, P. J. \& Bonnell, I. A. (2003) Orbital Migration and the Brown Dwarf Desert. In Brown Dwarfs, edited by E. Martín, vol. 211 of IAU Symposium.

Backer, D. C., Foster, R. S. \& Sallmen, S. (1993) A second companion of the millisecond pulsar 1620 - 26. Nature, 365, 817-819.

Baraffe, I., Chabrier, G., Allard, F. \& Hauschildt, P. H. (2002) Evolutionary models for low-mass stars and brown dwarfs: Uncertainties and limits at very young ages. $A \mathscr{E A}, \mathbf{3 8 2}, 563-572$.

Baran, A., Telting, J., Østensen, R., Winiarski, M., Drożdż, M., Kozię, D., Reed, M., Oreiro, R. eT AL. (2010) Constraining the degree of the dominant mode in QQ Vir. Ap\&SSS, 329, 199-203.

Barlow, B. N., Dunlap, B. H. \& Clemens, J. C. (2011a) Radial Velocity Confirmation of a Binary Detected from Pulse Timings. ApJ, 737, L2.

Barlow, B. N., Dunlap, B. H., Clemens, J. C., Reichart, D. E., Ivarsen, K. M., Lacluyze, A. P., Haislip, J. B. \& NyseWANDER, M. C. (2011b) Fortnightly fluctuations in the O-C diagram of CS 1246. $M N$ $R A S$, 414, 3434-3443.

Bear, E. \& Soker, N. (2011a) Connecting planets around horizontal branch stars with known exoplanets. MNRAS, 411, 1792-1802.
Bear, E. \& Soker, N. (2011b) Planets around Extreme Horizontal Branch Stars. In American Institute of Physics Conference Series, edited by S. Schuh, H. Drechsel, \& U. Heber, vol. 1331 of American Institute of Physics Conference Series.

Bear, E., Soker, N. \& Harpaz, A. (2011) Possible Implications of the Planet Orbiting the Red Horizontal Branch Star HIP 13044. ApJ, 733, L44.

Benatti, S., Silvotti, R., Claudi, R. U., Schuh, S., Lutz, R., Kim, S., Janulis, R., PAparò, M. ET AL. (2010) The EXOTIME project: a status report on PG 1325+101 (QQ Vir). ArXiv e-prints.

Beuermann, K., Buhlmann, J., Diese, J., Dreizler, S., Hessman, F. V., Husser, T., Miller, G. F., Nickol, N. Et AL. (2011) The giant planet orbiting the cataclysmic binary DP Leonis. $A \mathscr{E} A, \mathbf{5 2 6}, \mathrm{A} 53$.

Beuermann, K., Hessman, F. V., DreiZler, S., MARsh, T. R., PARsons, S. G., Winget, D. E., Miller, G. F., Schreiber, M. R. ET AL. (2010) Two planets orbiting the recently formed post-common envelope binary NN Serpentis. $A \mathscr{E} A, \mathbf{5 2 1}$, L60.

Bischoff, K., Tuparev, G., HessMAN, F. V. \& Nikolova, I. (2006) MONET/North: a very fast $1.2 \mathrm{~m}$ robotic telescope. In Society of Photo-Optical Instrumentation Engineers (SPIE) Conference Series, vol. 6270 of Society of Photo-Optical Instrumentation Engineers (SPIE) Conference Series.

Bowler, B. P., Johnson, J. A., Marcy, G. W., Henry, G. W., Peek, K. M. G., Fischer, D. A., Clubb, K. I., Liu, M. C. ET AL. (2010) Retired a Stars and Their Companions. III. Comparing the Mass-Period Distributions of Planets Around A-Type Stars and Sun-Like Stars. ApJ, 709, 396-410. 
Breger, M. (1981) The unusual Cepheid HR 7308 with a variable amplitude. ApJ, 249, 666-672.

Breger, M. \& Bischof, K. M. (2002) Close frequency pairs in Delta Scuti stars. $A \mathscr{E} A$, $\mathbf{3 8 5}, 537-545$.

Breger, M. \& Pamyatnykh, A. A. (2006) Amplitude variability or close frequencies in pulsating stars - the $\delta$ Scuti star FG Vir. $M N$ $R A S, \mathbf{3 6 8}, 571-578$.

Breger, M., Stich, J., Garrido, R., Martin, B., Jiang, S. Y., Li, Z. P., Hube, D. P., Ostermann, W. ET AL. (1993) Nonradial Pulsation of the Delta-Scuti Star BuCancri in the Praesepe Cluster. $A \& A, \mathbf{2 7 1}$, 482-486.

Buchler, J. R., Goupil, M. \& Hansen, C. J. (1997) On the role of resonances in nonradial pulsators. $A \& A, \mathbf{3 2 1}, 159-176$.

Buchler, J. R., Goupil, M. J. \& Serre, T. (1995) Dynamic pulsation amplitudes for a rotationally split nonradial mode: the $\mathrm{l}=1$ case. $A \mathscr{E} A$, 296, 405-417.

Burleigh, M. R., Steele, P. R., DobBie, P. D., Farihi, J., Napiwotzki, R., Maxted, P. F. L., Barstow, M. A., Jameson, R. F. ET AL. (2011) Brown Dwarf Companions to White Dwarfs. In American Institute of Physics Conference Series, edited by S. Schuh, H. Drechsel, \& U. Heber, vol. 1331 of American Institute of Physics Conference Series.

Charpinet, S. (2001) The pulsations in subdwarf B stars. Astronomische Nachrichten, 322, 387-393.

Charpinet, S., Fontaine, G. \& Brassard, P. (2009) Probing the driving region in hot subdwarf stars through nonadiabatic asteroseismology: the principle of the method. $A \mathscr{E} A, 493,595-600$.
Charpinet, S., Fontaine, G., Brassard, P., Chayer, P., Green, E. M. \& RanDALL, S. K. (2007) Ten years of asteroseismic modelling of pulsating B subdwarf stars: achievements, challenges, and prospects. Communications in Asteroseismology, 150, 241-246.

Charpinet, S., Fontaine, G., Brassard, P., Chayer, P., Rogers, F. J., Iglesias, C. A. \& Dorman, B. (1997) A Driving Mechanism for the Newly Discovered Class of Pulsating Subdwarf B Stars. ApJ, 483, L123L126.

Charpinet, S., Fontaine, G., Brassard, P. \& Dorman, B. (1996) The Potential of Asteroseismology for Hot, Subdwarf B Stars: A New Class of Pulsating Stars? ApJ, 471, L103-L106.

Charpinet, S., Fontaine, G., Brassard, P. \& Dorman, B. (2000) Adiabatic Survey of Subdwarf B Star Oscillations. I. Pulsation Properties of a Representative Evolutionary Model. ApJS, 131, 223-247.

Charpinet, S., Fontaine, G., Brassard, P. \& Dorman, B. (2002a) Adiabatic Survey of Subdwarf B Star Oscillations. II. Effects of Model Parameters on Pulsation Modes. ApJS, 139, 487-537.

Charpinet, S., Fontaine, G., Brassard, P. \& Dorman, B. (2002b) Adiabatic Survey of Subdwarf B Star Oscillations. III. Effects of Extreme Horizontal Branch Stellar Evolution on Pulsation Modes. ApJS, 140, 469-561.

Charpinet, S., Silvotti, R., Bonanno, A., Fontaine, G., Brassard, P., Chayer, P., Green, E. M., Bergeron, P. et Al. (2006) The rapidly pulsating subdwarf B star PG 1325+101. II. Structural parameters from asteroseismology. A\&A, 459, 565-576.

Classen, L., Geier, S., Heber, U. \& O'Toole, S. J. (2011) Radial Velocity Survey of Low-Mass Companions to sdB Stars. 
In American Institute of Physics Conference Series, edited by S. Schuh, H. Drechsel, \& U. Heber, vol. 1331 of American Institute of Physics Conference Series.

Clausen, D. \& Wade, R. A. (2011) How to Make a Singleton sdB Star via Accelerated Stellar Evolution. ApJ, 733, L42.

Dalessio, J., Provencal, J. L. \& ShipMAN, H. S. (2011) Searching for Planets with White Dwarf Pulsations: Spurious Detections. In American Institute of Physics Conference Series, edited by S. Schuh, H. Drechsel, \& U. Heber, vol. 1331 of American Institute of Physics Conference Series.

D'Cruz, N. L., Dorman, B., Rood, R. T. \& O'Connell, R. W. (1996) The Origin of Extreme Horizontal Branch Stars. ApJ, 466, 359-371.

de Pater, I. \& Lissauer, J. J. (2001) Planetary Sciences.

Dorman, B., Rood, R. T. \& O'Connell, R. W. (1993) Ultraviolet Radiation from Evolved Stellar Populations. I. Models. ApJ, 419, 596-614.

Dreizler, S., Schuh, S. L., Deetjen, J. L., Edelmann, H. \& Heber, U. (2002) HS0702+6043 - A new large amplitude sdB variable at the cool end of the instability region. $A \mathscr{E} A$, 386, 249-255.

Duncan, M. J. \& Lissauer, J. J. (1998) The Effects of Post-Main-Sequence Solar Mass Loss on the Stability of Our Planetary System. Icarus, 134, 303-310.

Eastman, J., Siverd, R. \& Gaudi, B. S. (2010) Achieving Better Than 1 Minute Accuracy in the Heliocentric and Barycentric Julian Dates. PASP, 122, 935-946.

FARIHI, J. (2011) Evidence for Terrestrial Planetary System Remnants at White Dwarfs. In American Institute of Physics Conference
Series, edited by S. Schuh, H. Drechsel, \& U. Heber, vol. 1331 of American Institute of Physics Conference Series.

Fontaine, G., Brassard, P., Charpinet, S., Green, E. M., Chayer, P., Billères, M. \& Randall, S. K. (2003) A Driving Mechanism for the Newly Discovered LongPeriod Pulsating Subdwarf B Stars. ApJ, 597, 518-534.

Fontaine, G., Brassard, P., Charpinet, S., Green, E. M., Chayer, P., RanDall, S. \& Dorman, B. (2006) Asteroseismology of hot B subdwarf stars: a brief overview. In Proceedings of $S O H O$ 18/GONG 2006/HELAS I, Beyond the spherical Sun, vol. 624 of ESA Special Publication.

Fontaine, G., Brassard, P., Charpinet, S., Green, E. M., Chayer, P., Randall, S. K. \& van Grootel, V. (2008) Achievements and Challenges in the Field of sdB Asteroseismology. In Hot Subdwarf Stars and Related Objects, edited by U. Heber, C. S. Jeffery, \& R. Napiwotzki, vol. 392 of Astronomical Society of the Pacific Conference Series.

Francoeur, M., Fontaine, G. \& Green, E. M. (2010) A major observational campaign on the hybrid pulsator HS0702+6043. Ap\&SS, 329, 189-192.

Geier, S., Edelmann, H., Heber, U. \& Morales-Rueda, L. (2009a) Discovery of a Close Substellar Companion to the Hot Subdwarf Star HD 149382 - The Decisive Influence of Substellar Objects on Late Stellar Evolution. ApJ, 702, L96-L99.

Geier, S., Heber, U., Edelmann, H., Kupfer, T., Napiwotzki, R. \& PodsiadLOWski, P. (2009b) Hot subdwarf binaries - Masses and nature of their heavy compact companions. Journal of Physics Conference Series, 172(1), 012008-012015.

Geier, S., Heber, U., Podsiadlowski, P., Edelmann, H., Napiwotzki, R., Kupfer, 
T. \& Müller, S. (2010) Hot subdwarf stars in close-up view. I. Rotational properties of subdwarf B stars in close binary systems and nature of their unseen companions. $A \mathscr{E} A$, 519, A25.

Geier, S., Heber, U., Tillich, A., Hirsch, H., Kupfer, T., Schaffenroth, V., Classen, L., Maxted, P. F. L. et al. (2011a) Substellar Companions and the Formation of Hot Subdwarf Stars. In American Institute of Physics Conference Series, edited by S. Schuh, H. Drechsel, \& U. Heber, vol. 1331 of American Institute of Physics Conference Series.

Geier, S., Schaffenroth, V., Drechsel, H., Heber, U., Kupfer, T., Tillich, A., Østensen, R. H., Smolders, K. et al. (2011b) Binaries Discovered by the MUCHFUSS Project: SDSS J08205+0008 - An Eclipsing Subdwarf B Binary with a Brown Dwarf Companion. ApJ, 731, L22.

Gourgouliatos, K. N. \& Jeffery, C. S. (2006) On the angular momentum evolution of merged white dwarfs. MNRAS, 371, 13811389.

Green, E. M., Fontaine, G., Hyde, E. A., For, B. \& Chayer, P. (2008) Systematics of Hot Subdwarfs Obtained from a Large Low Resolution Survey. In Hot Subdwarf Stars and Related Objects, edited by U. Heber, C. S. Jeffery, \& R. Napiwotzki, vol. 392 of Astronomical Society of the Pacific Conference Series.

Green, E. M., Fontaine, G., Reed, M. D., Callerame, K., Seitenzahl, I. R., White, B. A., Hyde, E. A., Østensen, R. ET AL. (2003) Discovery of A New Class of Pulsating Stars: Gravity-Mode Pulsators among Subdwarf B Stars. ApJ, 583, L31L34.

Greenstein, J. L. \& Sargent, A. I. (1974) The Nature of Faint Blue Stars in the Halo. II. ApJS, 28, 157-209.
Grether, D. \& Lineweaver, C. H. (2006) How Dry is the Brown Dwarf Desert? Quantifying the Relative Number of Planets, Brown Dwarfs, and Stellar Companions around Nearby Sun-like Stars. ApJ, 640, 1051-1062.

Guillot, T. (2010) On the radiative equilibrium of irradiated planetary atmospheres. $A \mathscr{E} A, \mathbf{5 2 0}, \mathrm{A} 27$.

Han, Z., Podsiadlowski, P., Maxted, P. F. L. \& Marsh, T. R. (2003) The origin of subdwarf B stars - II. MNRAS, 341, 669-691.

Han, Z., Podsiadlowski, P., Maxted, P. F. L., Marsh, T. R. \& Ivanova, N. (2002) The origin of subdwarf B stars - I. The formation channels. MNRAS, 336, 449-466.

Handler, G. (2003) Merging Data from Large and Small Telescopes - Good or Bad? And: How Useful is the Application of Statistical Weights to Time-Series Photometric Measurements? Baltic Astronomy, 12, 253-270.

Heber, U. (1986) The atmosphere of subluminous B stars. II - Analysis of 10 helium poor subdwarfs and the birthrate of sdB stars. A $\mathcal{E} A, \mathbf{1 5 5}, 33-45$.

Heber, U. (2009) Hot Subdwarf Stars. ARAESA, 47, 211-251.

Hessman, F. V. (2001) MONET: a MOnitoring NEtwork of Telescopes. In IAU Colloq. 183: Small Telescope Astronomy on Global Scales, edited by B. Paczynski, W.-P. Chen, \& C. Lemme, vol. 246 of Astronomical Society of the Pacific Conference Series.

Hu, H., Nelemans, G., Østensen, R., Aerts, C., Vučković, M. \& Groot, P. J. (2007) An evolutionary study of the pulsating subdwarf B eclipsing binary PG 1336-018 (NY Virginis). AEAA, 473, 569-577. 
Humason, M. L. \& Zwicky, F. (1947) A Search for Faint Blue Stars. ApJ, 105, 8591.

Husser, T.-O. (2011) Claude - An Automation Tool for the Monet Telescopes. ArXiv eprints.

Ivanova, N. (2011) Common envelope: the progress and the pitfalls. ArXiv e-prints.

Jacobs, V. A., Østensen, R. H., van Winckel, H., Bloemen, S., PÁpics, P. I., Raskin, G., Debosscher, J., UtTEnthaler, S. ET AL. (2011) HERMES High-Resolution Spectroscopy of HD 149382Where Did the Planet Go? In American Institute of Physics Conference Series, edited by S. Schuh, H. Drechsel, \& U. Heber, vol. 1331 of American Institute of Physics Conference Series.

Jeffery, C. S. \& Saio, H. (2006a) Fe-bump instability: the excitation of pulsations in subdwarf $\mathrm{B}$ and other low-mass stars. $M N$ $R A S, \mathbf{3 7 1}, 659-672$.

Jeffery, C. S. \& Saio, H. (2006b) Gravitymode pulsations in subdwarf B stars: a critical test of stellar opacity. MNRAS, 372, L48L52.

Jones, M. I., Jenkins, J. S. \& Rojo, P. (2011) Study of Planetary Systems around Giant Stars. In American Institute of Physics Conference Series, edited by S. Schuh, H. Drechsel, \& U. Heber, vol. 1331 of American Institute of Physics Conference Series.

KaWAler, S. D. (2010) Structure and evolution of pulsating hot subdwarfs. Astronomische Nachrichten, 331, 1020-1025.

Kawaler, S. D. \& Hostler, S. R. (2005) Internal Rotation of Subdwarf B Stars: Limiting Cases and Asteroseismological Consequences. ApJ, 621, 432-444.

Kepler, S. O. (1993) Whole Earth Telescope data analysis. Baltic Astronomy, 2, 515-529.
Kepler, S. O., Costa, J. E. S., Castanheira, B. G., Winget, D. E., Mullally, F., NAther, R. E., Kilic, M., vON HipPEL, T. ET AL. (2005) Measuring the Evolution of the Most Stable Optical Clock G 117-B15A. ApJ, 634, 1311-1318.

Kepler, S. O., Winget, D. E., Nather, R. E., Bradley, P. A., Grauer, A. D., Fontaine, G., Bergeron, P., Vauclair, G. ET AL. (1991) A detection of the evolutionary time scale of the DA white dwarf G117 - B15A with the Whole Earth Telescope. ApJ, 378, L45-L48.

Kilkenny, D. (2010) Amplitude variations in pulsating sdB stars. ApESSS, 329, 175-181.

Kilkenny, D., Fontaine, G., Green, E. M. \& SchuH, S. (2010) A Proposed Uniform Nomenclature for Pulsating Hot Subdwarf Stars. Information Bulletin on Variable Stars, 5927, 1-3.

Kilkenny, D., Koen, C., O'Donoghue, D. \& Stobie, R. S. (1997) A new class of rapidly pulsating star - I. EC 14026-2647, the class prototype. MNRAS, 285, 640-644.

Kilkenny, D., Stobie, R. S., O’Donoghue, D., Koen, C., Hambly, N., MacGillivray, H. \& Lynas-Gray, A. E. (2006) Three new pulsating sdB stars from the Edinburgh-Cape survey. MNRAS, 367, 1603-1608.

Kruspe, R., Schuh, S., Silvotti, R. \& Traulsen, I. (2008) Spectroscopy of the sdB pulsator HS 2201+2610. Communications in Asteroseismology, 157, 325-327.

Kurtz, D. W. (2005) Asteroseismology: Past, Present and Future. Journal of Astrophysics and Astronomy, 26, 123-138.

Lee, J. W., Kim, S., Kim, C., Кoch, R. H., Lee, C., Kim, H. \& Park, J. (2009) The $\mathrm{sdB}+\mathrm{M}$ Eclipsing System HW Virginis and its Circumbinary Planets. AJ, 137, 31813190. 
Lenz, P. \& Breger, M. (2004) Period04: A software package to extract multiple frequencies from real data. In IAU Symposium, edited by J. Zverko, J. Ziznovsky, S. J. Adelman \& W. W. Weiss.

Lenz, P. \& Breger, M. (2005) Period04 User Guide. Communications in Asteroseismology, 146, 53-136.

Livio, M. \& Soker, N. (2002) The Effects of Planets and Brown Dwarfs on Stellar Rotation and Mass Loss. ApJ, 571, L161-L164.

LÖPtien, B. (2009) Analyse der Pulsationsfrequenzen des sdB Sterns HS $0444+0458$. Bachelorarbeit, Georg-August-Universität Göttingen.

Lutz, R. (2007) Lightcurve analyses of pulsating sdB stars. Diplomarbeit, Georg-AugustUniversität Göttingen.

Lutz, R., Schun, S. \& Silvotti, R. (2011) The EXOTIME Targets HS 0702+6043 and HS 0444+0458. In American Institute of Physics Conference Series, edited by S. Schuh, H. Drechsel, \& U. Heber, vol. 1331 of American Institute of Physics Conference Series.

Lutz, R., Schuh, S., Silvotti, R., Bernabei, S., Dreizler, S., Stahn, T. \& Hügelmeyer, S. D. (2009) The planethosting subdwarf B star V 391 Pegasi is a hybrid pulsator. $A \mathscr{E} A, 496,469-473$.

Lutz, R., Schuh, S., Silvotti, R., DreiZler, S., Green, E. M., Fontaine, G., Stahn, T., Hügelmeyer, S. D. et al. (2008) Light Curve Analysis of the Hybrid SdB PulsatorsHS $0702+6043$ and HS 2201+2610. In Hot Subdwarf Stars and Related Objects, edited by U. Heber, C. S. Jeffery, \& R. Napiwotzki, vol. 392 of Astronomical Society of the Pacific Conference Series.

Marcy, G. W., Butler, R. P., Vogt, S. S., Fischer, D. A., Wright, J. T.,
Johnson, J. A., Tinney, C. G., Jones, H. R. A. ET AL. (2008) Exoplanet properties from Lick, Keck and AAT. Physica Scripta Volume T, 130(1), 014001-014007.

Markwardt, C. B. (2009) Non-linear Leastsquares Fitting in IDL with MPFIT. In $A s$ tronomical Data Analysis Software and Systems XVIII, edited by D. A. Bohlender, D. Durand, \& P. Dowler, vol. 411 of Astronomical Society of the Pacific Conference Series.

Maxted, P. F. L., Heber, U., Marsh, T. R. \& NorTh, R. C. (2001) The binary fraction of extreme horizontal branch stars. MNRAS, 326, 1391-1402.

Maxted, P. F. L., Napiwotzki, R., DobBie, P. D. \& Burleigh, M. R. (2006) Survival of a brown dwarf after engulfment by a red giant star. Nature, 442, 543-545.

Mazeh, T. \& Faigler, S. (2010) Detection of the ellipsoidal and the relativistic beaming effects in the CoRoT-3 lightcurve. $A \mathscr{E} A, \mathbf{5 2 1}$, L59.

Montgomery, M. H. \& O'Donoghue, D. (1999) A derivation of the errors for least squares fitting to time series data. Delta Scuti Star Newsletter, 13, 28-32.

Mullally, F., Reach, W. T., De GenNAro, S. \& Burrows, A. (2009) Spitzer Planet Limits Around the Pulsating White Dwarf GD66. ApJ, 694, 327-331.

Mullally, F., Winget, D. E., De Gennaro, S., Jeffery, E., Thompson, S. E., Chandler, D. \& Kepler, S. O. (2008) Limits on Planets around Pulsating White Dwarf Stars. ApJ, 676, 573-583.

Mullally, F. R. (2007) Substellar companions to white dwarves. Ph.D. thesis, The University of Texas at Austin. 
Müller, S., Geier, S. \& Heber, U. (2010) The cool companion of AA Doradus - Brown dwarf or late M star? ApESSS, 329, 101-105.

Napiwotzki, R., Karl, C. A., Lisker, T., Heber, U., Christlieb, N., Reimers, D., Nelemans, G. \& Homeier, D. (2004) Close binary EHB stars from SPY. ApESSS, 291, 321-328.

Nelemans, G. \& Tauris, T. M. (1998) Formation of undermassive single white dwarfs and the influence of planets on late stellar evolution. $A \mathscr{E} A$, 335, L85-L88.

Omiya, M., Han, I., Izumiura, H., Lee, B., Sato, B., Kim, K., Yoon, T. S., KamBe, E. ET AL. (2011) Korean-Japanese Planet Search Program: Substellar Companions around Intermediate-Mass Giants. In American Institute of Physics Conference Series, edited by S. Schuh, H. Drechsel, \& U. Heber, vol. 1331 of American Institute of Physics Conference Series.

Østensen, R., Heber, U., Silvotti, R., Solheim, J., Dreizler, S. \& Edelmann, H. (2001a) Four new subdwarf B pulsators. $A \mathscr{E} A, \mathbf{3 7 8}, 466-476$.

Østensen, R., Solheim, J.-E., Heber, U., Silvotti, R., Dreizler, S. \& Edelmann, H. (2001b) Detection of pulsations in three subdwarf B stars. A\&A A, 368, 175-182.

Østensen, R. H. (2006) The Subdwarf Database: Released. Baltic Astronomy, 15, 85-90.

Østensen, R. H. (2009) Asteroseismology and Evolution of EHB stars. Communications in Asteroseismology, 159, 75-87.

Østensen, R. H., Oreiro, R., Hu, H., Drechsel, H. \& HeBer, U. (2008) HS 2231+2441: An Eclipsing sdB Binary with a Substellar Companion. In Hot Subdwarf Stars and Related Objects, edited by U. Heber, C. S. Jeffery, \& R. Napiwotzki, vol. 392 of
Astronomical Society of the Pacific Conference Series.

Østensen, R. H., Oreiro, R., Solheim, J., Heber, U., Silvotti, R., GonzálezPérez, J. M., Ulla, A., Pérez HernánDEZ, F. ET AL. (2010) A survey for pulsating subdwarf B stars with the Nordic Optical Telescope. A\&A, 513, A6.

Østensen, R. H., Silvotti, R., Charpinet, S., Oreiro, R., Bloemen, S., Baran, A. S., Reed, M. D., Kawaler, S. D. ET AL. (2011) First Kepler results on compact pulsators - VI. Targets in the final half of the survey phase. MNRAS, 414, 2860-2870.

O'Toole, S., Heber, U., Geier, S., Classen, L. \& De Marco, O. (2011) Radial Velocity Search for Substellar Companions to sdB Stars. In American Institute of Physics Conference Series, edited by S. Schuh, H. Drechsel, \& U. Heber, vol. 1331 of American Institute of Physics Conference Series.

O'Toole, S. J., Heber, U. \& Benjamin, R. A. (2004) Detection of a companion to the pulsating sdB Feige 48. A\&SA, 422, 10531058.

Pajdosz, G. (1995a) Non-evolutionary secular period increase in pulsating DA white dwarfs. $A \& A$, 295, L17-L19.

Pajdosz, G. (1995b) The Effect of Proper Motion on $\mathrm{P}$ in Pulsating Stars. In IAU Colloq. 155: Astrophysical Applications of Stellar Pulsation, edited by R. S. Stobie \& P. A. Whitelock, vol. 83 of Astronomical Society of the Pacific Conference Series.

Percy, J. R., Matthews, J. M. \& Wade, J. D. (1980) Period changes in dwarf Cepheids. A\&A, 82, 172-176.

Perets, H. B. (2010) Second generation planets. ArXiv e-prints. 
Perets, H. B. (2011) Planets in Evolved Binary Systems. In American Institute of Physics Conference Series, edited by S. Schuh, H. Drechsel, \& U. Heber, vol. 1331 of American Institute of Physics Conference Series.

Perryman, M. A. C. (2000) Extra-solar planets. Reports on Progress in Physics, 63, 12091272 .

Podsiadlowski, P. (2008) The Evolution of Close Binaries. In Astronomical Society of the Pacific Conference Series, edited by A. Evans, M. F. Bode, T. J. O'Brien, \& M. J. Darnley, vol. 401 of Astronomical Society of the Pacific Conference Series.

Podsiadlowski, P. \& Han, Z. (2004) The Progenitors of sdB Binaries: Confronting Theory with Observations. ApESSS, 291, 291-298.

Podsiadlowski, P., Han, Z., Lynas-Gray, A. E. \& Brown, D. (2008) Hot Subdwarfs in Binaries as the Source of the FarUV Excess in Elliptical Galaxies. In Hot Subdwarf Stars and Related Objects, edited by U. Heber, C. S. Jeffery, \& R. Napiwotzki, vol. 392 of Astronomical Society of the Pacific Conference Series.

Politano, M., TAam, R. E., van DeR Sluys, M. \& Willems, B. (2008) CommonEnvelope Mergers: A Possible Channel for Forming Single sdB Stars. ApJ, 687, L99L102.

Politano, M. \& Weiler, K. P. (2007) Population Synthesis Studies of Close Binary Systems Using a Variable Common Envelope Efficiency Parameter. I. Dependence on Secondary Mass. ApJ, 665, 663-679.

Qian, S., Dai, Z., Liao, W., Zhu, L., Liu, L. \& Zhaо, E. G. (2009a) A Substellar Companion to the White Dwarf-Red Dwarf Eclipsing Binary NN Ser. ApJ, 706, L96-L99.
Qian, S., LiaO, W., Zhu, L. \& Dai, Z. (2010) Detection of a Giant Extrasolar Planet Orbiting the Eclipsing Polar DP Leo. ApJ, 708, L66-L68.

Qian, S., Zhu, L., Zola, S., Liao, W., LiU, L., Li, L., Winiarski, M., Kuligowska, E. ET AL. (2009b) Detection of a Tertiary Brown Dwarf Companion in the sdB-Type Eclipsing Binary HS 0705+6700. ApJ, 695, L163-L165.

Quirrenbach, A., Reffert, S. \& Bergmann, C. (2011) Planets around Giant Stars. In American Institute of Physics Conference Series, edited by S. Schuh, H. Drechsel, \& U. Heber, vol. 1331 of American Institute of Physics Conference Series.

Randall, S. K., Fontaine, G., Brassard, P. \& Bergeron, P. (2005) The Potential of Multicolor Photometry for Pulsating Subdwarf B Stars. ApJS, 161, 456-479.

Randall, S. K., van Grootel, V., Fontaine, G., Charpinet, S. \& BrasSARD, P. (2009) Observations and asteroseismological analysis of the rapid subdwarf $\mathrm{B}$ pulsator EC 09582-1137. A \&A, 507, 911-921.

Rasio, F. A., Tout, C. A., Lubow, S. H. \& Livio, M. (1996) Tidal Decay of Close Planetary Orbits. ApJ, 470, 1187-1191.

Reed, M. D., Kawaler, S. D., Østensen, R. H., Bloemen, S., Baran, A., Telting, J. H., Silvotti, R., Charpinet, S. ET AL. (2010) First Kepler results on compact pulsators - III. Subdwarf B stars with V1093 Her and hybrid (DW Lyn) type pulsations. MNRAS, 409, 1496-1508.

Reed, M. D., Kawaler, S. D., Zola, S., Jiang, X. J., Dreizler, S., Schuh, S. L., Deetjen, J. L., Kalytis, R. ET AL. (2004) Observations of the pulsating subdwarf B star Feige 48: Constraints on evolution and companions. MNRAS, 348, 1164-1174. 
Reed, M. D. \& Stiening, R. (2004) A Search for Main-Sequence Companions to Subdwarf B Stars Using the Two Micron All Sky Survey. $P A S P, \mathbf{1 1 6}, 506-515$.

Reed, M. D., Terndrup, D. M., Zhou, A., Unterborn, C. T., An, D. \& EgGen, J. R. (2007) Resolving the pulsations of subdwarf B stars: HS 0039+4302, HS 0444+0458 and an examination of the group properties of resolved pulsators. MNRAS, 378, 1049-1063.

Rybicki, K. R. \& Denis, C. (2001) On the Final Destiny of the Earth and the Solar System. Icarus, 151, 130-137.

Saio, H. \& Jeffery, C. S. (2000) The evolution of a rapidly accreting helium white dwarf to become a low-luminosity helium star. $M N$ $R A S, \mathbf{3 1 3}, 671-677$.

Schaffenroth, V., Geier, S., Heber, U., Drechsel, H., Østensen, R. H., Maxted, P. F. L., Kupfer, T. \& BarLOw, B. N. (2011) Analysis of Two Eclipsing Hot Subdwarf Binaries with a Low Mass Stellar and a Brown Dwarf Companion. In American Institute of Physics Conference Series, edited by S. Schuh, H. Drechsel, \& U. Heber, vol. 1331 of American Institute of Physics Conference Series.

Schröder, K.-P. \& Connon Smith, R. (2008) Distant future of the Sun and Earth revisited. MNRAS, 386, 155-163.

SchuH, S. (2010) Pulsations and planets: The asteroseismology-extrasolar-planet connection. Astronomische Nachrichten, 331, 489-501.

Schuh, S., Huber, J., Dreizler, S., Heber, U., O'Toole, S. J., Green, E. M. \& Fontaine, G. (2006) HS 0702+6043: a star showing both short-period p-mode and longperiod g-mode oscillations. A\&A, 445, L31L34.
Schun, S., Kruspe, R., Lutz, R. \& Silvotti, R. (2009) Time-resolved spectroscopy of the planet-hosting sdB pulsator V391 Pegasi. Communications in Asteroseismology, 159, 91-93.

Schun, S., Silvotti, R., Lutz, R., Loeptien, B., Green, E. M., Østensen, R. H., LeCCIA, S., KIM, S. ET AL. (2010) EXOTIME: searching for planets around pulsating subdwarf B stars. ApESSS, 329, 231-242.

Schuh, S. L., Dreizler, S., Deetuen, J. L. \& Göhler, E. (2003) TRIPP: An Aperture Photometry Package for the Reduction of CCD Time Series Images. Baltic Astronomy, 12, 167-182.

Seidelmann, P. K. \& Fukushima, T. (1992) Why new time scales? $A \& A$, 265, 833-838.

Setiawan, J., Klement, R., Henning, T., Rix, H., Rochau, B., Schulze-Hartung, T. \& Rodmann, J. (2011) A Planetary Companion around a Metal-Poor Star with Extragalactic Origin. In American Institute of Physics Conference Series, edited by S. Schuh, H. Drechsel, \& U. Heber, vol. 1331 of American Institute of Physics Conference Series.

Silvotti, R. (2008) The Subdwarf B + Giant Planet System V391 Peg: Different Scenarios for its Previous Evolution. In Hot Subdwarf Stars and Related Objects, edited by U. Heber, C. S. Jeffery, \& R. Napiwotzki, vol. 392 of Astronomical Society of the Pacific Conference Series.

Silvotti, R. (2009) Late-stage stellar evolution: impact on planets. International Journal of Astrobiology, 8, 241-242.

Silvotti, R., Bonanno, A., Bernabei, S., Fontaine, G., Charpinet, S., Leccia, S., KJeldsen, H., Janulis, R. ET AL. (2006) The rapidly pulsating subdwarf $B$ star $P G$ $1325+101$. I. Oscillation modes from multisite observations. A\&A, 459, 557-564. 
Silvotti, R., Janulis, R., Schuh, S. L., Charpinet, S., Oswalt, T., Silvestri, N., Gonzalez Perez, J. M., Kalytis, R. ET AL. (2002a) The temporal spectrum of the sdB pulsating star HS $2201+2610$ at 2 ms resolution. AधA A, 389, 180-190.

Silvotti, R., Østensen, R., Heber, U., Solheim, J., Dreizler, S. \& Altmann, M. (2002b) PG 1325+101 and PG 2303+019: Two new large amplitude subdwarf $\mathrm{B}$ pulsators. $A \& A, \mathbf{3 8 3}, 239-243$.

Silvotti, R., Randall, S. K., Dhillon, V. S., Marsh, T. R., Savoury, C. D., Schuh, S., Fontaine, G. \& Brassard, P. (2010) V391 Peg: Identification of the two main pulsation modes from ULTRACAM u'g'r' amplitudes. Astronomische Nachrichten, 331, 1034-1037.

Silvotti, R., Schuh, S., Janulis, R., Solheim, J., Bernabei, S., Østensen, R., Oswalt, T. D., Bruni, I. ET AL. (2007) A giant planet orbiting the 'extreme horizontal branch' star V391 Pegasi. Nature, 449, 189-191.

Silvotti, R., Szabó, R., Degroote, P., Østensen, R. H. \& Schuh, S. (2011) The Potential of the Timing Method to Detect Evolved Planetary Systems. In American Institute of Physics Conference Series, edited by S. Schuh, H. Drechsel, \& U. Heber, vol. 1331 of American Institute of Physics Conference Series.

Soker, N. (1996) What Planetary Nebulae Can Tell Us about Planetary Systems. ApJ, 460, L53-L56.

Soker, N. (1998) Can Planets Influence the Horizontal Branch Morphology? AJ, 116, 1308-1313.

Stark, M. A. \& Wade, R. A. (2003) Single and Composite Hot Subdwarf Stars in the Light of 2MASS Photometry. AJ, 126, 14551471.
Sterken, C. (2005) The O-C Diagram: Basic Procedures. In The Light-Time Effect in Astrophysics: Causes and cures of the $O-C$ diagram, edited by C. Sterken, vol. 335 of $A s$ tronomical Society of the Pacific Conference Series.

Sweigart, A. V. \& Gross, P. G. (1978) Evolutionary sequences for red giant stars. $A p J S$, 36, 405-437.

Telting, J. H. \& Østensen, R. H. (2004) Radial-velocity and line-profile variations in the sdBV star PG 1325+101. A\& A, 419, 685-693.

Thejll, P., Flynn, C., Williamson, R. \& SAFfer, R. (1997) Proper motions of the hot subdwarfs. The kinematic population membership of the sdB. A\&SA, 317, 689-693.

Thorsett, S. E., Arzoumanian, Z. \& TayLOR, J. H. (1993) PSR B1620-26 - A binary radio pulsar with a planetary companion? ApJ, 412, L33-L36.

Tillich, A., Heber, U., Geier, S., Hirsch, H., Maxted, P. F. L., GäNSicke, B. T., Marsh, T. R., Napiwotzki, R. ET AL. (2011) The Hyper-MUCHFUSS project: probing the Galactic halo with sdB stars. $A \mathscr{E} A, \mathbf{5 2 7}, \mathrm{A} 137$.

Torres, G. (2010) On the Use of Empirical Bolometric Corrections for Stars. AJ, 140, 1158-1162.

Van Grootel, V., Charpinet, S., Fontaine, G., Brassard, P., Green, E. M., Randall, S. K., Silvotti, R., Østensen, R. H. et AL. (2010) Early Asteroseismic Results from Kepler: Structural and Core Parameters of the Hot B Subdwarf KPD $1943+4058$ as Inferred from g-mode Oscillations. ApJ, 718, L97-L101.

Villaver, E. (2011) The Fate of Planets. In American Institute of Physics Conference Series, edited by S. Schuh, H. Drechsel, \& 
U. Heber, vol. 1331 of American Institute of Physics Conference Series.

Winget, D. E., Nather, R. E., Clemens, J. C., Provencal, J., Kleinman, S. J., Bradley, P. A., Wood, M. A., Claver, C. F. ET AL. (1990) Whole earth telescope observations of the white dwarf G29-38 Phase variations of the 615 second period. ApJ, 357, 630-637.

Wolszczan, A. (1994) Confirmation of EarthMass Planets Orbiting the Millisecond Pulsar PSR B1257+12. Science, 264, 538-542.

Wolszczan, A. \& Frail, D. A. (1992) A planetary system around the millisecond pulsar PSR1257 + 12. Nature, 355, 145-147.

Wright, J. T., Fakhouri, O., Marcy, G. W., Han, E., Feng, Y., Johnson, J. A., Howard, A. W., Fischer, D. A. ET AL. (2011) The Exoplanet Orbit Database. PASP, 123, 412-422.

Zhou, A. (1999) A review of the O-C method and period change. Publications of the Beijing Astronomical Observatory, 33, 17-33.

Zorotovic, M., Schreiber, M. R., GänSicke, B. T. \& Nebot Gómez-Morán, A. (2010) Post-common-envelope binaries from SDSS. IX: Constraining the commonenvelope efficiency. $A \mathscr{E} A, \mathbf{5 2 0}, \mathrm{A} 86$. 



\section{Appendix A}

\section{Finding charts and field of view snapshots}

The finding charts in the top panels of the following Figures show the EXOTIME targets (encircled and labeled by their respective name) and possible stable reference stars (encircled). If the FOV of the observing instrument allowed it, it was tried to always use the same reference stars.

The bottom panels of the following Figures show actual single snapshops which were taken during an arbitrary observational run. The snaphots of HS 0702+6043, HS 0444+0458, PG 1325+101 and HS 2201+2610 were taken with the MONET/N $1.2 \mathrm{~m}$ telescope and have a fixed field of view of roughly 5'x5'. The snapshot of EC 09582-1137 was taken with the Calar Alto $2.2 \mathrm{~m}$ telescope, where a windowing mode was used.

To match the orientations, the upper panel has to be rotated clockwise by 90 degrees against the lower panel. 


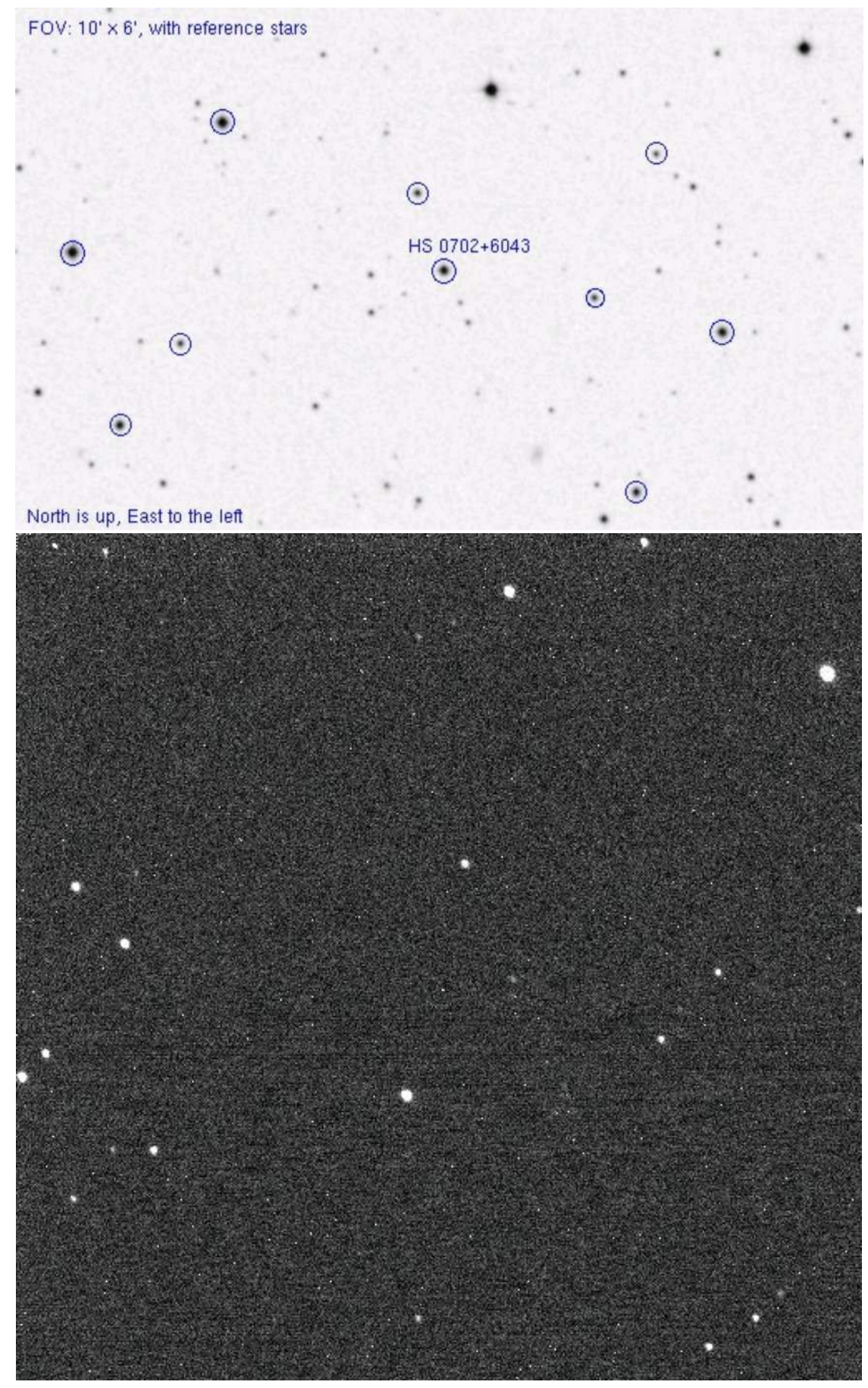

Figure A.1: Finding chart (top) and FOV snapshot (bottom) for HS 0702+6043. 
FOV: $7^{\prime} \times 4^{\prime}$, with reference stars

\section{HS0444+0458}

$\odot$

$\odot$

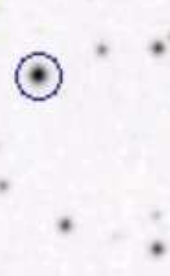

$\odot$

North is up, East to the left

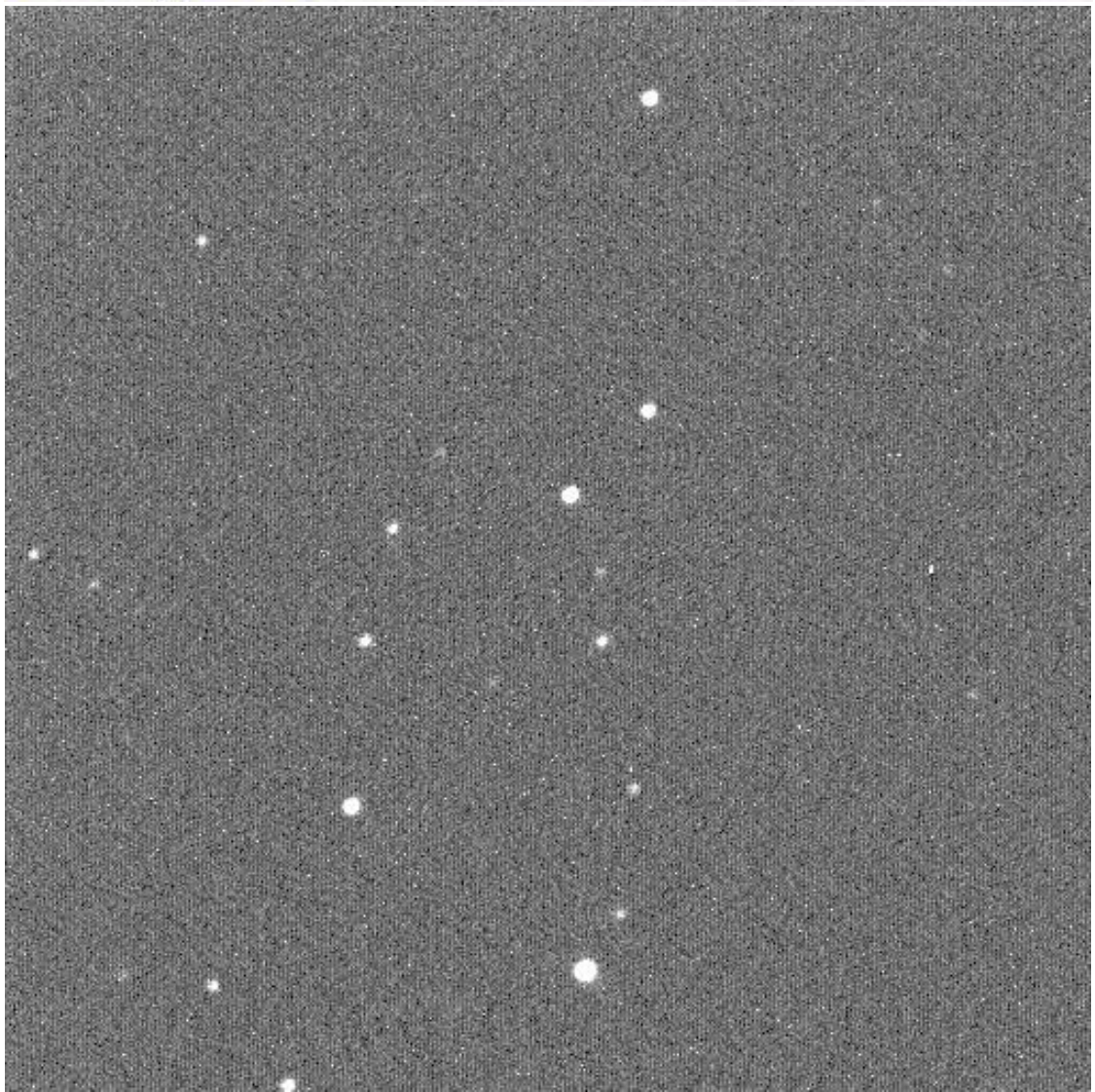

Figure A.2: Finding chart (top) and FOV snapshot (bottom) for HS 0444+0458. 
FOV: $5^{\prime} \times 3^{\prime}$, with reference star

PG1325+101

$\odot$

North is up, East to the left

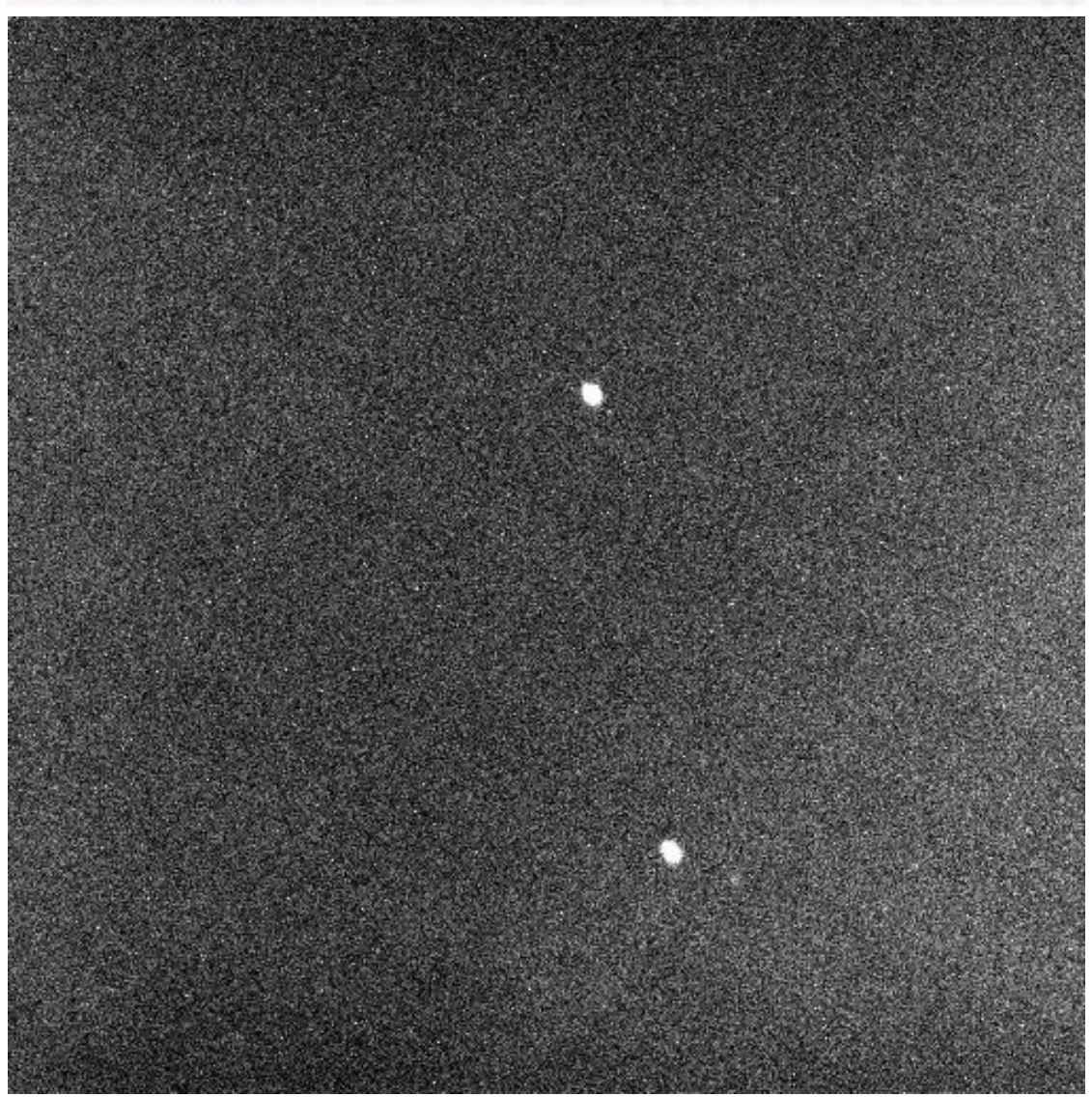

Figure A.3: Finding chart (top) and FOV snapshot (bottom) for PG 1325+101. 


\section{FOV: $6^{\prime} \times 4^{*}$, with reference stars}

$\therefore 9$

HS $2201+2610$
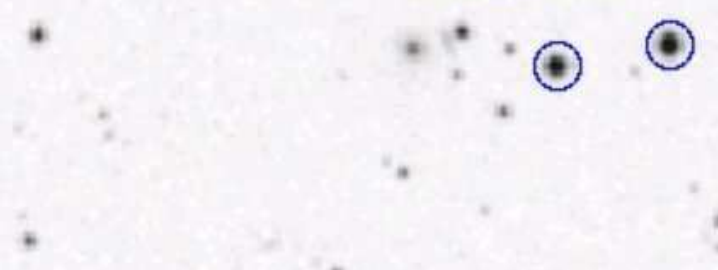

(C)

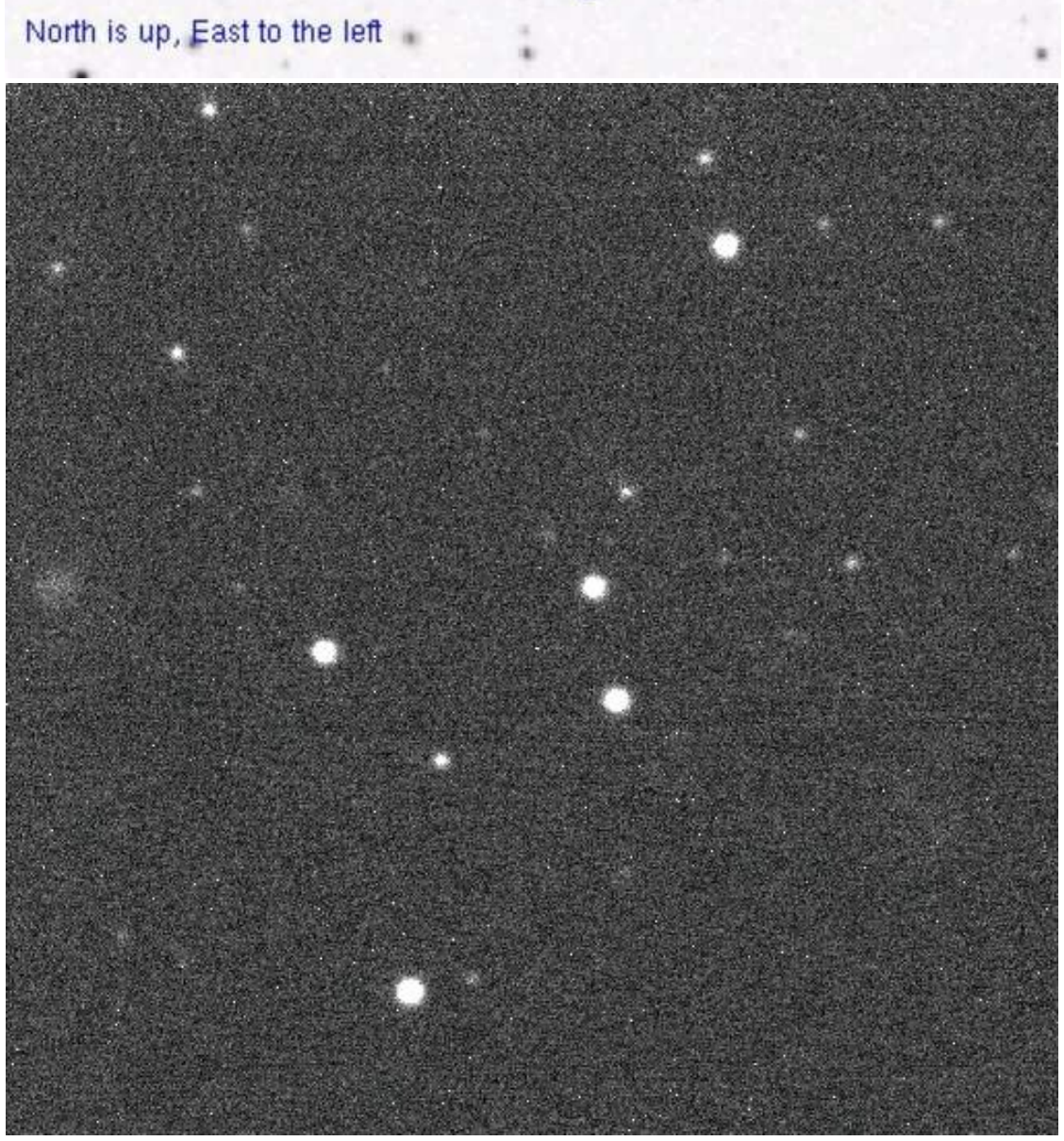

Figure A.4: Finding chart (top) and FOV snapshot (bottom) for HS 2201+2610. 

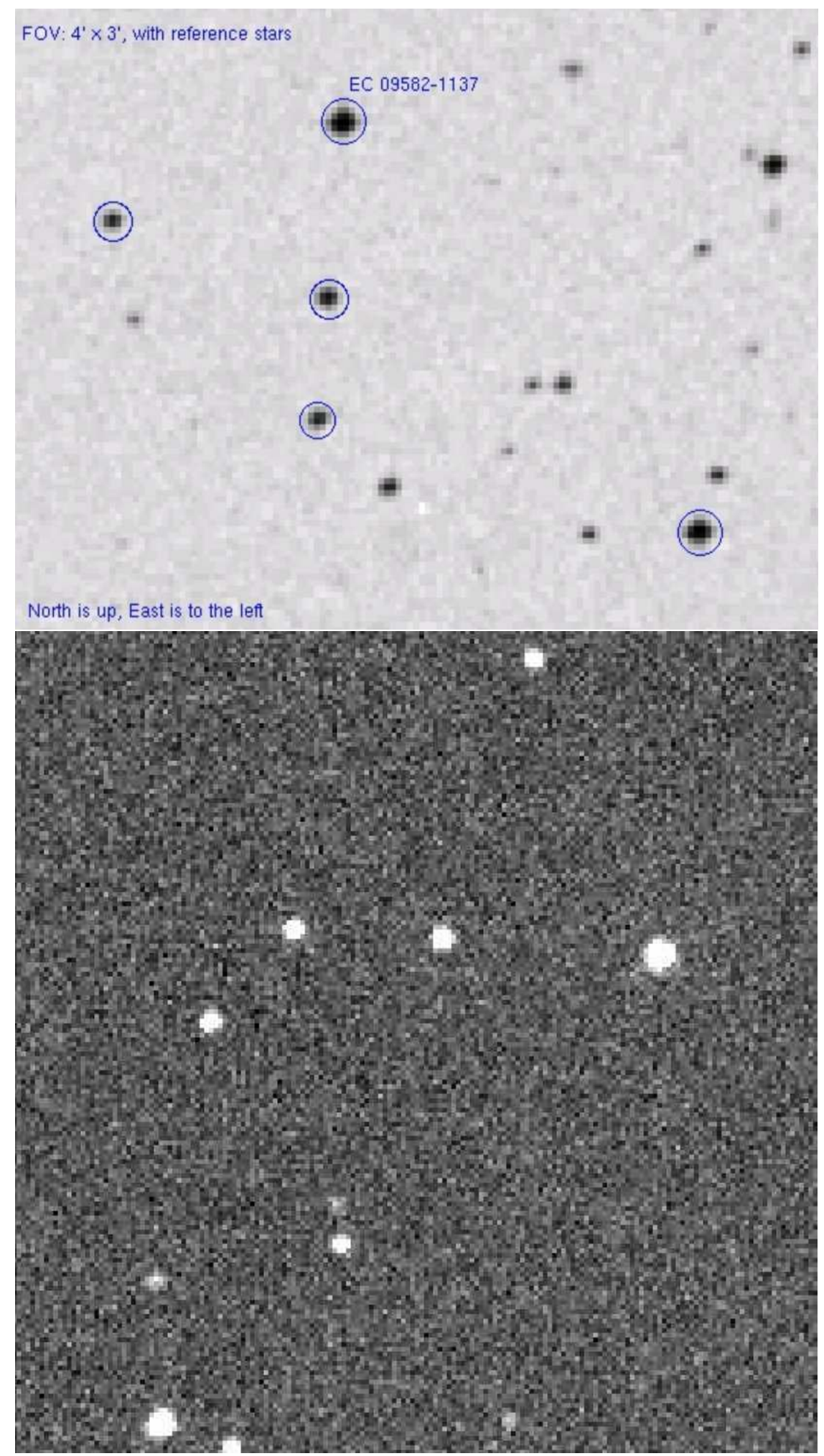

Figure A.5: Finding chart (top) and FOV snapshot (bottom) for EC 09582-1137. 


\section{Appendix B}

\section{Example light curves}

The following example lightcurves (roughly between 1 hour and 5 hours in length) are intended to illustrate the different qualities of some instruments. 

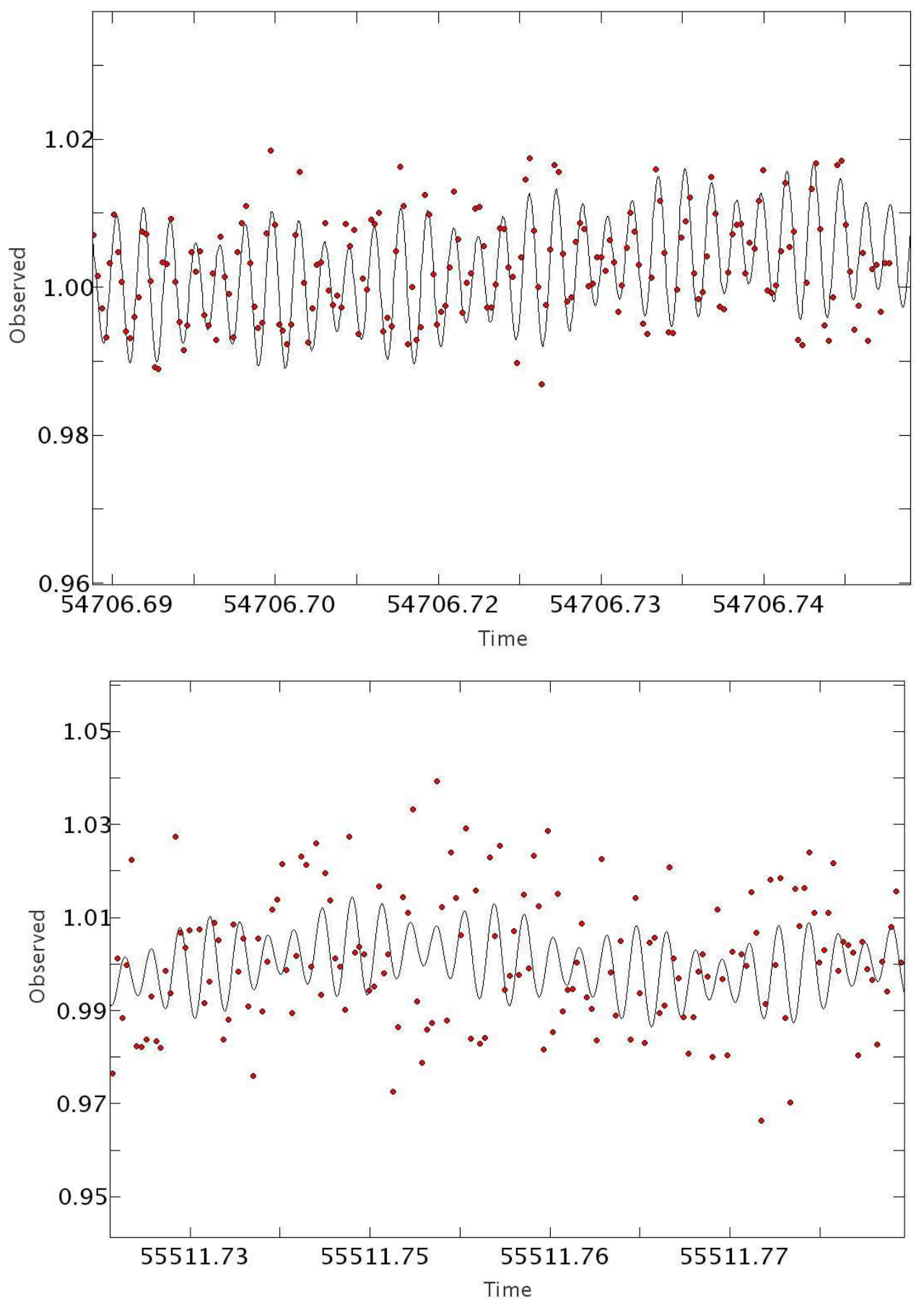

Figure B.1: Light curve examples for HS 0444+0458 with the TNG telescope (top) and with MONET/N (bottom). 

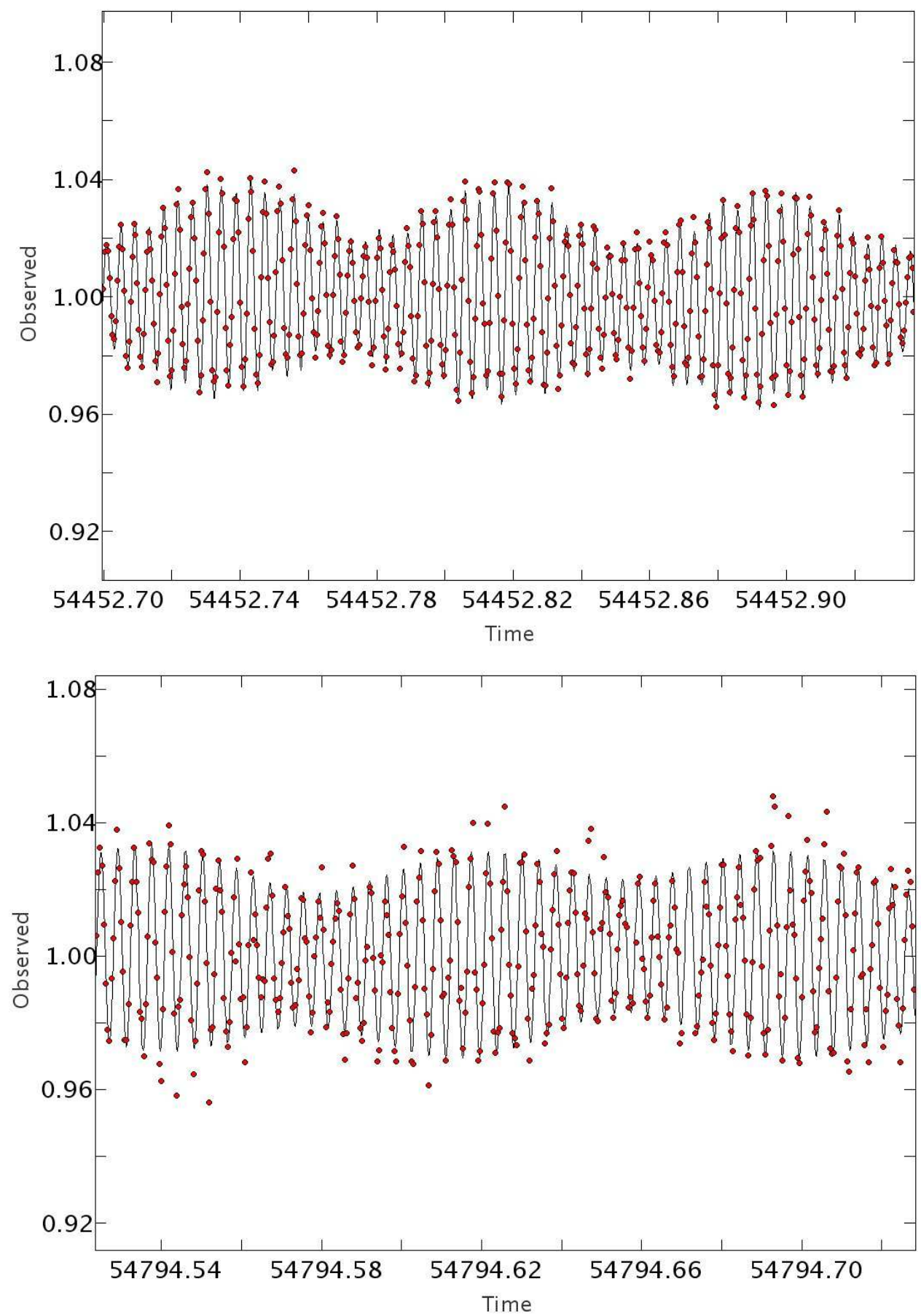

Figure B.2: Light curve examples for HS 0702+6043 with the MtB telescope (top) and with CA (bottom). 


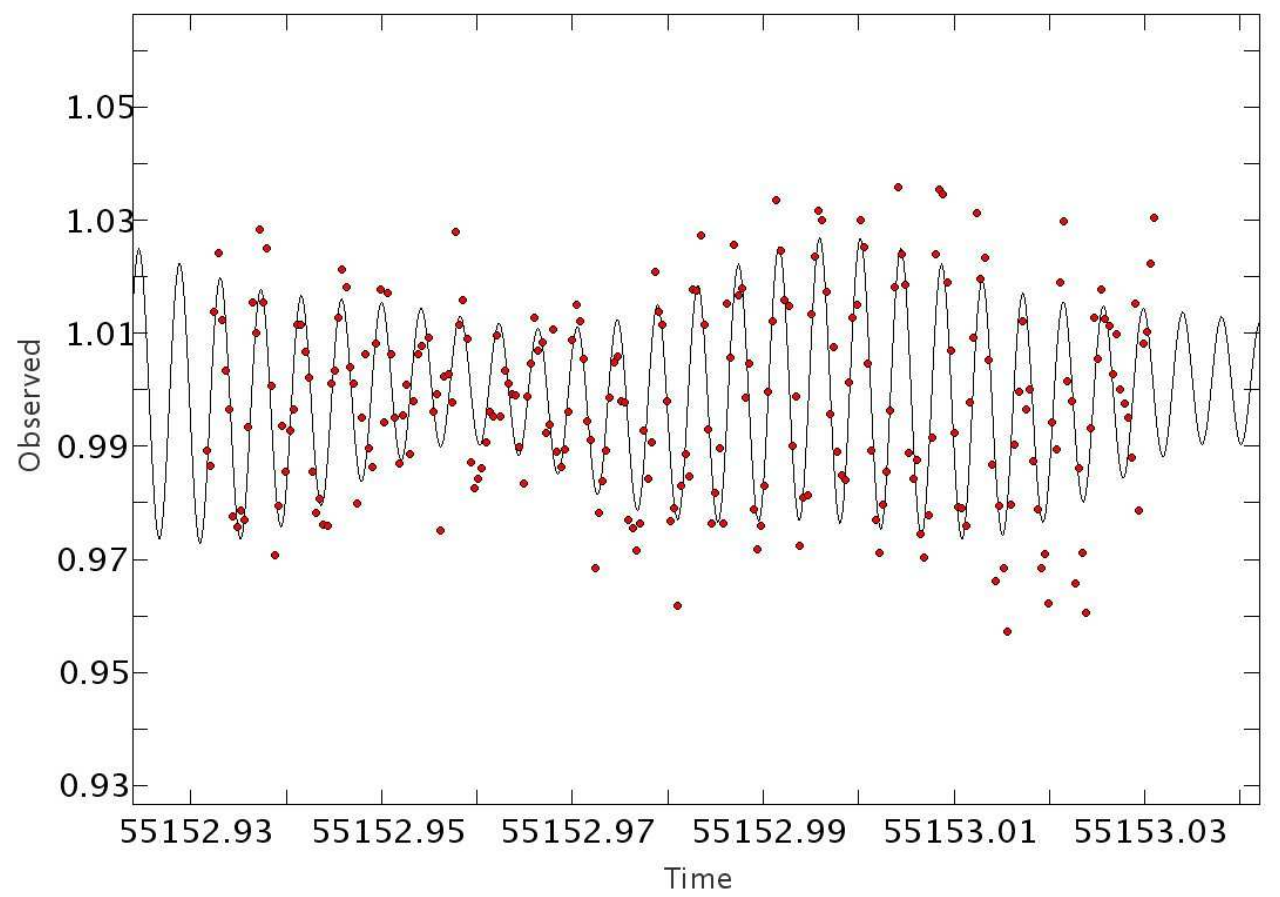

Figure B.3: Light curve example for HS 0702+6043 with the MONET/N telescope. 


\section{Appendix C}

\section{Example periodograms}

The following example periodograms show the dependence of the frequency resolution on the lenght of the observations. See Figure caption for further explanation. 

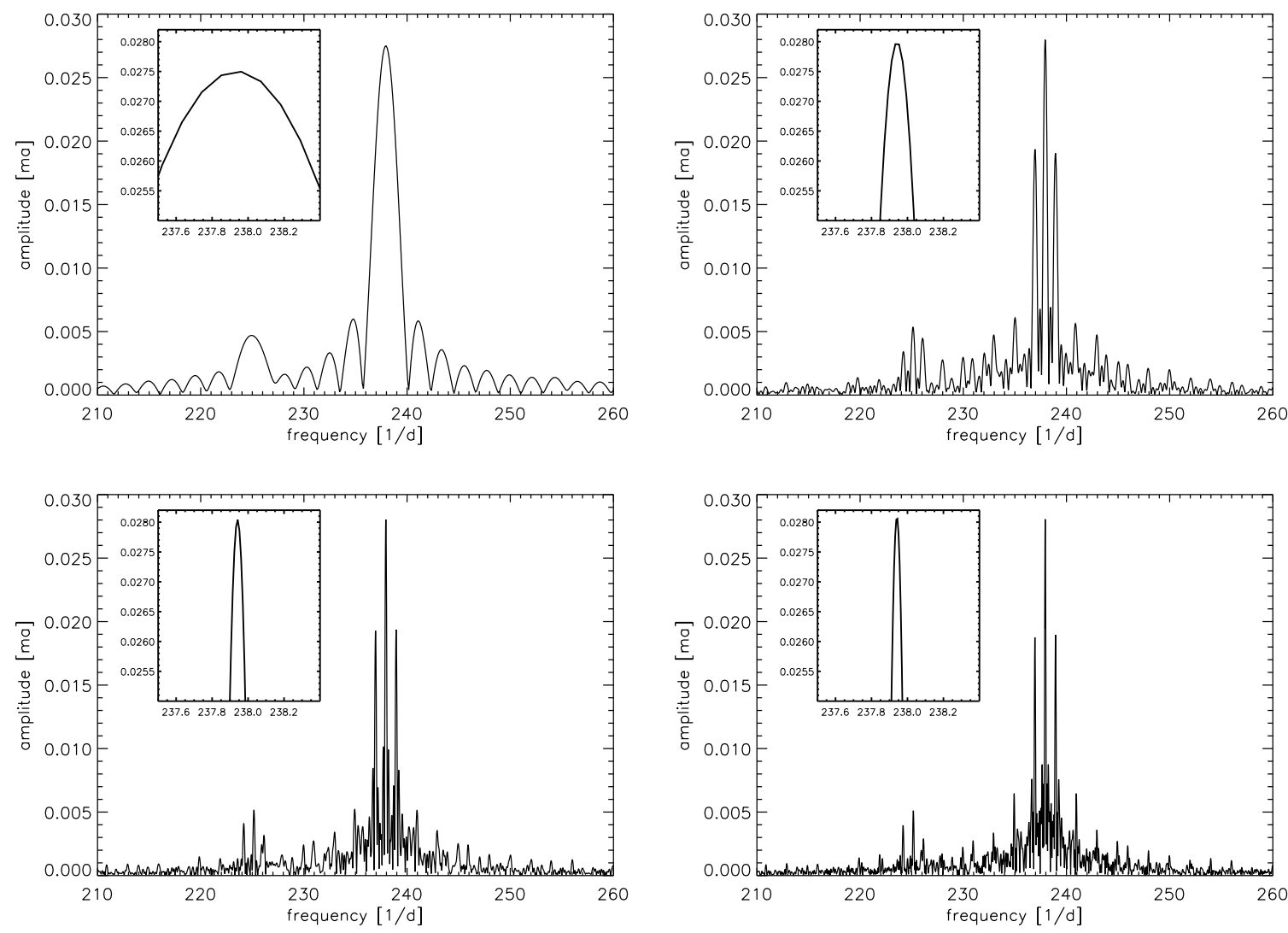

Figure C.1: Examples for Fourier transforms of different temporal resolutions for our target HS 0702+6043 based on a continuous MtB data set from 2008-0111 to 2008-01-19. The FTs zoom into the region of the two strongest frequencies (around $2381 / \mathrm{d}$ and $2251 / \mathrm{d}$ ) and are intended to illustrate the increase of temporal resolution as the time base increases. The top left is based on one night of data, the top right on three consecutive nights of data, the bottom left on six consecutive nights and the bottom right on nine consecutive nights of data. The inset windows show a zoom into the main peak. 


\section{Appendix D}

\section{List of substellar companions to sdB stars}

The following Tables provide a collection of all exoplanetary and brown dwarf companions to sdB stars as well as controversial cases which are still under debate. 


\begin{tabular}{|c|c|c|c|c|}
\hline host star type & substellar companion & companion name & detection method & reference \\
\hline \multicolumn{5}{|l|}{ single sdB stars: } \\
\hline sdBV & $\mathrm{P}$ & HS $2201+2610 b$ & pulsation timing & Silvotti et al. (2007) \\
\hline sdBV & $\mathrm{P}$ & HS $0702+6043$ b & pulsation timing & this thesis \\
\hline $\mathrm{sdB}$ & $\mathrm{BD}$ & SDSS J08205+0008 b & RV + eclipse phot. & Geier et al. (2011b) \\
\hline sdBV & $\mathrm{BD}$ & HS $0444+0458$ b & pulsation timing & this thesis \\
\hline \multicolumn{5}{|l|}{ binary sdB stars: } \\
\hline $\mathrm{sdB}+\mathrm{dM}$ & $\mathrm{P}$ & HW Vir c & eclipse timing & Lee et al. (2009) \\
\hline $\mathrm{sdB}+\mathrm{dM}$ & $\mathrm{BD}$ & HW Vir b & eclipse timing & Lee et al. (2009) \\
\hline $\mathrm{sdB}+\mathrm{dM}$ & $\mathrm{BD}$ & HS $0705+6700 \mathrm{~b}$ & eclipse timing & Qian et al. (2009b) \\
\hline
\end{tabular}


Table D.2: List of candidates to sdB stars where it is controversial if the object is substellar or if it exists at all. EP: eclipse photometry.

\begin{tabular}{|c|c|c|c|c|}
\hline $\begin{array}{r}\text { stellar } \\
\text { type }\end{array}$ & $\begin{array}{r}\text { stellar } \\
\text { name }\end{array}$ & comment & $\begin{array}{r}\text { detection } \\
\text { method }\end{array}$ & reference \\
\hline \multirow[t]{2}{*}{$\mathrm{sdB}+?$} & HD 149382 & planet detection by Geier et al. (2009a), & RV & Geier et al. (2009a) \\
\hline & & but non-detection by Jacobs et al. (2011) & $\mathrm{RV}$ & Jacobs et al. (2011) \\
\hline $\mathrm{sdOB}+?$ & AA Dor & companion is $\mathrm{BD}$ or low mass star & $\mathrm{RV}+\mathrm{EP}$ & Müller et al. (2010) \\
\hline $\mathrm{sdB}+?$ & HS $2231+2441$ & companion is substellar for a low sdB mass & $\mathrm{RV}+\mathrm{EP}$ & Østensen et al. (2008) \\
\hline $\mathrm{sdB}+?$ & SDSS J162256+473051 & object is under analysis & $\mathrm{RV}$ & Schaffenroth et al. (2011) \\
\hline
\end{tabular}





\section{Acknowledgements / Danksagungen}

First and foremost I want to thank all observers and facilities who contributed to the data archive of the EXOTIME program. Amongst others, the work in this thesis

- is based on data obtained with the MOnitoring NEtwork of Telescopes (MONET), funded by the "Astronomie \& Internet" program of the Alfried Krupp von Bohlen und Halbach Foundation, Essen, and operated by the Georg-August- Universität Göttingen, the McDonald Observatory of the University of Texas at Austin, and the South African Astronomical Observatory

- is based on observations collected at the Centro Astronómico Hispano Alemán (CAHA) at Calar Alto, operated jointly by the Max-Planck Institut für Astronomie and the Instituto de Astrofísica de Andalucía (CSIC)

- is based on observations made with the Italian Telescopio Nazionale Galileo (TNG) operated on the island of La Palma by the Fundación Galileo Galilei of the INAF (Instituto Nazionale di Astrofisica) at the Spanish Observatorio del Roque de los Muchachos of the Instituto de Astrofisica de Canarias - has made use of the Exoplanet Orbit Database and the Exoplanet Data Explorer at exoplanets.org.

I am deeply grateful to Betsy Green and her collaborators, who kindly provided the data of the extensive Mt. Bigelow campaign on HS 0702+6043 with the permission to include these in our O-C analysis.

I want to express my gratitude to Roberto Silvotti for allowing me to be a part of the EXOTIME collaboration and to trust me with the analysis of the targets HS $0444+0458$ and HS $0702+6043$. I also appreciate Roberto's helpful suggestions and comments on various parts of the thesis manuscript which helped a lot to improve it. 
Mein besonderer Dank gilt in allererster Linie Sonja Schuh und Stefan Dreizler sowohl für die hervorragende Betreuung während der letzten Jahre, als auch für das Referieren dieser Arbeit. Eure kompetente Hilfsbereitschaft und eure Fähigkeit neue Impulse zu geben, haben mich stets motiviert.

Ich danke Sonja für ihre unzähligen Korrekturlesungen, Kommentare, Hilfen und Vorschläge, ohne denen diese Arbeit niemals in ihrer jetzigen Form zustandegekommen wäre. Ebenso danke ich Rick für das Korrekturlesen dieser Arbeit mit besonderem Augenmerk auf sprachliche Aspekte.

Die "technical support crew" des MONET/N Teleskops verdient ein ganz besonderes Lob. Ulf, Markus und Rick haben mir etliche male die Beobachtungsnacht gerettet, indem sie MONET nach allen Regeln der Kunst dazu überredeten doch wieder mit mir zu sprechen (obwohl MONET in diesen Momenten eigentlich gerade so gar nicht in der richtigen "Stimmung" für eine Beobachtungsnacht mit mir war). Dabei mussten Ulf und Markus den ein oder anderen nächtlichen Anruf meinerseits ertragen. Sorry dafür.

Den ehemaligen und aktuellen Mitgliedern der Abteilung "stellare Astrophysik", sowie dem stets hilfsbereiten Sekretariat danke ich für die nette Arbeitsatmosphäre.

Dieter Schmitt hatte stets ein offenes Ohr für meine Fragen und Anliegen bezüglich der "International Max Planck Research School", was ich sehr zu schätzen weiss.

Ich möchte bei allen, die ich eventuell vergessen oder nicht namentlich genannt habe, um Entschuldigung bitten.

Des weiteren möchte ich mich herzlich bei meinem engsten Freundeskreis (ihr wisst schon selbst wer alles gemeint ist) bedanken. Eure Freundschaft ist unbezahlbar.

Schlussendlich gilt der größte Dank meiner Familie. Eure Unterstützung in allen denkbaren Momenten macht mich extrem stolz. Diese Arbeit widme ich meiner Mum, Ute, die in jeder Lage das wichtigste Vorbild für mich ist. 


\section{Curriculum Vitae}

Dipl.-Phys. Ronny Bernd Lutz

Stumpfe Eiche 77

37077 Göttingen

Geburtstag:

10.11.1981

Geburtsort:

Fürstenhagen

Staatsangehörigkeit:

Deutsch

08/1988 - 07/1992 Grundschule Fürstenhagen

08/1992 - 07/1994 Orientierungsstufe der Freiherr-vom-Stein

Gesamtschule Hessisch-Lichtenau

08/1994 - 06/2001 Gymnasiale Oberstufe der Freiherr-vom-Stein Gesamtschule Hessisch-Lichtenau

01.06.2001 allgemeine Hochschulreife

10/2001 - 08/2007 Physikstudium (Georg-August-Universität Göttingen)

23.10.2003 Vordiplom Physik (Georg-August-Universität Göttingen)

22.08.2007 Diplom Physik (Georg-August-Universität Göttingen)

12/2007 - 08/2011 Promotionsstipendiat

(International Max Planck Research School, IMPRS) 